\title{
Diversity of the Marine Cyanobacterium Trichodesmium: Characterization of the Woods Hole Culture Collection and Quantification of Field Populations
}

\author{
by
}

Annette Michelle Hynes

B.S., University of Nebraska-Lincoln (1998)

Submitted to the Department of Biology

in partial fulfillment of the requirements for the degree of

Doctor of Philosophy in Biological Oceanography

at the

MASSACHUSETTS INSTITUTE OF TECHNOLOGY

and the

WOODS HOLE OCEANOGRAPHIC INSTITUTION

September 2009

(c) Annette Michelle Hynes, MMIX. All rights reserved.

The author hereby grants to MIT and WHOI permission to reproduce and distribute publicly paper and electronic copies of this thesis document in whole or in part.

Author

Department of Biology

August 28, 2009

Certified by

Scott C. Doney

Senior Scientist

Thesis Supervisor

Certified by

John B. Waterbury

Scientist Emeritus

Thesis Supervisor

Accepted by

Simon Thorrold

Chair, Joint Committee for Biological Oceanography 


\title{
Diversity of the Marine Cyanobacterium Trichodesmium: Characterization of the Woods Hole Culture Collection and Quantification of Field Populations
}

by

\author{
Annette Michelle Hynes
}

\author{
Submitted to the Department of Biology \\ on August 28, 2009, in partial fulfillment of the \\ requirements for the degree of \\ Doctor of Philosophy in Biological Oceanography
}

\begin{abstract}
Trichodesmium is a colonial, $\mathrm{N}_{2}$-fixing cyanobacterium found in tropical oceans. Species of Trichodesmium are genetically similar but several species exist together in the same waters. In order to coexist, Trichodesmium spp. may occupy different niche spaces through differential utilization of resources such as nutrients and light, and through responses to physical characteristics such as temperature and turbulence. To investigate niche differentiation in Trichodesmium, I characterized cultured strains of Trichodesmium, identified and enumerated Trichodesmium clades in the field, and investigated $\mathrm{P}$ stress and $\mathrm{N}_{2}$ fixation in field populations. Species of Trichodesmium grouped into two clades based on sequences from 16S rDNA, the internal transcribed spacer (ITS), and the heterocyst differentiation gene hetR. Clade I contained Trichodesmium erythraeum and Trichodesmium contortum, and clade II contained Trichodesmium thiebautii, Trichodesmium tenue, Trichodesmium hildebrandtii, and Trichodesmium pelagicum. Each clade was morphologically diverse, but species within each clade had similar pigmentation. I developed a quantitative polymerase chain reaction (qPCR) method to distinguish between these two clades. In field populations of the Atlantic and Pacific Oceans, the qPCR method revealed that clade II Trichodesmium spp. were more prominent than clade I in the open ocean. Concentrations of Trichodesmium did not correlate with nutrient concentrations, but clade I had wider temperature and depth distributions than clade II. Temperature and light are physical characteristics that may define niche spaces for species of Trichodesmium. Clade I and II concentrations correlated with each other in the Pacific but not in the Atlantic, indicating that the two clades were limited by the same factors in the Pacific while different factors were limiting the abundance of the two clades in the Atlantic. Trichodesmium populations in the North Atlantic were more P stressed and had higher $\mathrm{N}_{2}$ fixation rates than populations in the western Pacific. While nutrient concentrations didn't directly correlate with Trichodesmium concentrations, the contrasting nutrient regimes found in the Atlantic and Pacific Oceans might influence distributions of the two clades differently. Unraveling the differences among species of Trichodesmium
\end{abstract}


begins to explain their coexistence and enables us to understand factors controlling global $\mathrm{N}_{2}$ fixation.

Thesis Supervisor: Scott C. Doney

Title: Senior Scientist

Thesis Supervisor: John B. Waterbury

Title: Scientist Emeritus 


\section{Dedication}

I dedicate this thesis to my students from the Kiundwani Secondary School class of 2003, Machakos District, Kenya.

“Elimu maisha si vitabu.” (Methali za Kiswahili)

"Education is life, not books." (Swahili proverb) 


\section{Acknowledgements}

Throughout my graduate career, I have been supported by the National Science Foundation (NSF) Biocomplexity Program Grant (OCE-0323332); the Center for Microbial Oceanography Research and Education (C-MORE), an NSF Science and Technology Center (EF-0424599); the Woods Hole Oceanographic Institution (WHOI) Ocean Life Institute (OLI) grant to J. Waterbury, and the WHOI Academic Programs Office.

I would like to thank my advisors, Scott Doney and John Waterbury of WHOI. Scott has supported me from day one as a scientist and as a human. While we haven't been able to complete the modeling we originally set out to do, he has been invaluable in helping me to view my data from new angles. John was very generous in taking me in when I needed a new lab and in sharing with me his skill and patience in culturing the fickle and wonderful cyanobacteria.

My committee members have been indispensable with their molecular expertise: Eric Webb of the University of Southern California (USC), Tracy Mincer of WHOI, and Edward DeLong of the Massachusetts Institute of Technology (MIT). Having Eric at WHOI was like having a bonus advisor, and his enthusiasm for science is infectious, even over the phone to USC. Tracy was a highly welcome arrival at WHOI, and on many occasions he has saved me from weeks of banging my head against a problem with qPCR. Ed had the benefit of being more removed from my project and could see the larger picture of my challenges with wisdom and a sense of humor. Judith McDowell (WHOI) served as the chair of both my thesis proposal defense and my thesis defense, and I thank Judy for keeping everybody in line and for serving as a role model for me.

I am eternally grateful to the administrative assistants, the Academic Programs Office at WHOI, and the Joint Program Office at MIT for making it easier to dot the i's and cross the t's, and for giving their assistance with genuine love and concern.

I would like to thank my plethora of WHOI labmates over the years, who have been helpful with their myriad of knowledge: P. Dreux Chappell, Sarah Cooley, Ian Ehren- 
reich, Sheean Haley, Whitney Krey, Justin Ladner, Naomi Levine, Emily Lorch, Misty Miller, Elizabeth Orchard, Adam Rivers, Emmanuel Vásquez-Rivera, Brian Wilson, and Louie Wurch. I especially want to thank Freddy Valois, whose skills are always in high demand, and Nan Trowbridge, who made everything run smoothly (usually by greasing the wheels with baked goods).

I am indebted to Dave Glover for graciously taking the MATLAB and LATEXchallenges I have brought before him, Tim Shank for allowing me to use his thermal cycler and his labspace for sequencing, Rhian Waller (currently University of Hawai'i (UH)) for teaching me the ropes of sequencing, Rebecca Gast for allowing me to make an obnoxious amount of noise in her laboratory by using her beadbeater, Sonya Dyhrman for being my initial biology advisor, Heidi Sosik for her advice on image processing, the residents of Watson Laboratory for rampant sharing of equipment and ideas, and Mark Dennett and Alexi Shalopyonok for teaching me flow cytometery in my early days of working with Crocosphaera, all from WHOI. I would like to thank Penny Chisholm and Ed DeLong for the use of their thermal cycler and robot at MIT where I did the majority of my qPCR, and Rex Malmstrom, who was very patient and generous with his time in teaching me to use these machines.

This thesis would not have been possible without the support of C-MORE. In addition to funding, I have had access to a community of microbial ecologists from $\mathrm{UH}$, Oregon State University (OSU), University of California-Santa Cruz (UCSC), Monterey Bay Aquarium Research Institute (MBARI), MIT, and WHOI with a wide variety of backgrounds and experiences. Graduate students, post-doctoral scholars, and principal investigators have all taken the time to share their expertise with me, especially to help me troubleshoot my technical problems or to provide field samples.

I want to acknowledge the members of "Team Tricho," fellow students and postdocs who study this finicky and fantastic organism Trichodesmium: P. Dreux Chappell (WHOI, currently University of Rhode Island), Carolyn Holl (Georgia Institute of Technology, currently Oceanic Institute), Elizabeth Orchard (WHOI), Jill Sohm (USC), and 
Angelicque White (OSU). I want to thank you for the countless conversations, your feedback on ideas, your help on cruises, and your friendship. I especially want to thank Dreux who has been a dear friend and my biggest cheerleader through this whole PhD process.

Lastly, I want to thank my family, both born and chosen. Thank you, Kate Buckman, for being my best friend, roommate, and comic relief. Thank you, Joe Confalone, for being my emotional support, my extra-curricular activity, and my occasional laboratory assistant. And thank you, Mom, Pops, Jeannette, Lynnette, and Raennette, for your love, nurturing, and being my roots. 


\section{Contents}

1 Introduction 19

Statement of Purpose . . . . . . . . . . . . . . . . . . . . . 19

Ecological roles of Trichodesmium . . . . . . . . . . . . . . . . . 20

Species identifications and distributions $\ldots \ldots \ldots \ldots \ldots \ldots$

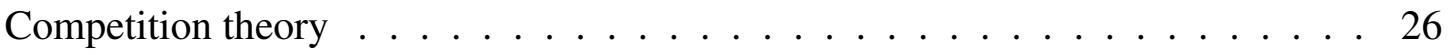

Niche differentiation in cyanobacteria $\ldots \ldots \ldots$

Overview of thesis chapters . . . . . . . . . . . . . . 31

2 Diversity of the $\mathbf{N}_{2}$-fixing Cyanobacterium Trichodesmium: Characterization of the Woods Hole Culture Collection 35

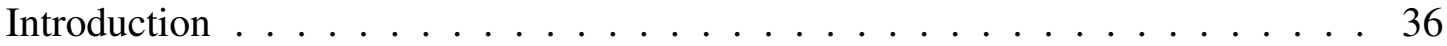

Identification of Trichodesmium $\ldots \ldots \ldots$. . . . . . . . 36

Photosynthetic pigments . . . . . . . . . . . . . . 37

Characterization of the Woods Hole culture collection . . . . . . . . . . 40

Methods . . . . . . . . . . . . . . . . . . . . . 42

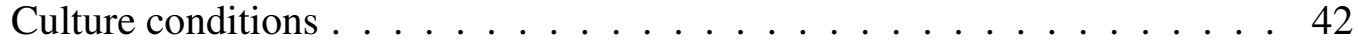

Micrographs . . . . . . . . . . . . . . . . . . 42

Phycobiliprotein extraction and absorption spectra . . . . . . . . . . 42

Sequences and phylogeny . . . . . . . . . . . . . . . . 43

Results . . . . . . . . . . . . . . . . . . . . 45

Genetic characterization of Trichodesmium . . . . . . . . . . . 45 
Cell morphology . . . . . . . . . . . . . . . . . 60 60

Absorption spectra of phycobiliproteins $\ldots \ldots \ldots \ldots$

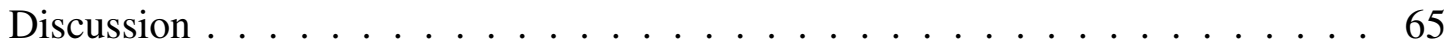

3 Development of a quantitative polymerase chain reaction (qPCR) assay to distinguish the two clades of Trichodesmium 71

Introduction . . . . . . . . . . . . . . . . . . . . 72

Methods . . . . . . . . . . . . . . . . . . . . . . 74

Primer design and preparation of cloned qPCR standards $\ldots \ldots \ldots$. . . 74

Serial dilutions and cell counts of Trichodesmium cultures . . . . . . . 76

DNA extraction . . . . . . . . . . . . . . . . 77

Quantitative polymerase chain reaction (qPCR) $\ldots \ldots \ldots \ldots$

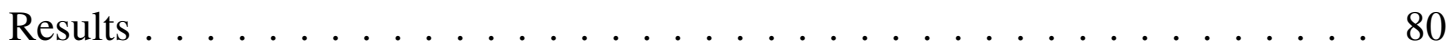

qPCR assay development $\ldots \ldots \ldots \ldots \ldots \ldots$

DNA extractions and serial dilutions $\ldots \ldots \ldots \ldots \ldots$

Discussion . . . . . . . . . . . . . . . 86

4 The Distribution of Trichodesmium field populations $\quad 89$ Introduction . . . . . . . . . . . . . . . . . . . . 90

Methods . . . . . . . . . . . . . . . . . . . . . 91

Sample collection, storage, and extraction . . . . . . . . . . 91

Quantitative polymerase chain reaction (qPCR) . . . . . . . 93

Results . . . . . . . . . . . . . . . . . . . . . . 94

Transect hydrography, nutrients, and qPCR _ . . . . . . . . . . 94

Nutrients and Trichodesmium . . . . . . . . . . . . . . . . . 100

Temperature, depth and Trichodesmium . . . . . . . . . . . 100

Melting types, temperature, and depth $\ldots \ldots \ldots \ldots 7$

West Pacific warm pool diel cycle $\ldots \ldots \ldots$. . . . . . . 110

Discussion . . . . . . . . . . . . . . . . . . . . 112 
5 Cross-basin comparison of phosphorus stress and nitrogen fixation in Trichodesmium 119 Introduction . . . . . . . . . . . . . . . . . . . . . 120

New nitrogen . . . . . . . . . . . . . . . . 120

Phosphorus and iron $\ldots \ldots \ldots \ldots \ldots \ldots \ldots \ldots$

Alkaline phosphatase . . . . . . . . . . . . . . 122

Methods . . . . . . . . . . . . . . . . . . . . . . 123

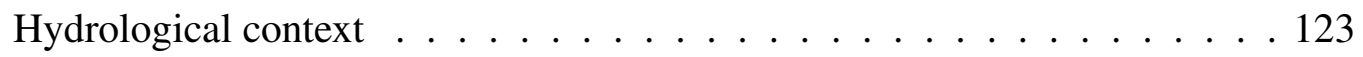

Collection of colonies . . . . . . . . . . . . . . . . . . . . . . 124

Enzyme-labeled fluorescence $(\mathrm{ELF}) \ldots \ldots \ldots$

Nutrient Analysis . . . . . . . . . . . . . . . . . . . 125

Nitrogenase activity . . . . . . . . . . . . . . 126

Results . . . . . . . . . . . . . . . . . . . . . . . . . . . . . . . 127

Physical conditions . . . . . . . . . . . . . . . . 127

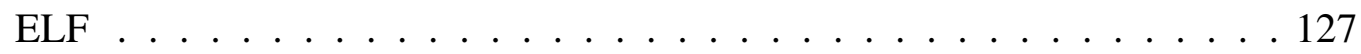

DIP and ELF $\ldots \ldots \ldots \ldots \ldots \ldots \ldots \ldots \ldots \ldots \ldots \ldots \ldots \ldots$

Nitrogenase activity $\ldots \ldots \ldots \ldots \ldots \ldots \ldots \ldots$

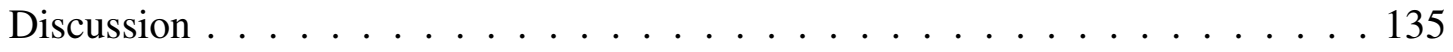

6 Concluding remarks $\quad 143$

Thesis summary . . . . . . . . . . . . . . . . . . . . . 143

Evolution of Trichodesmium _ . . . . . . . . . . . . . . . . . . 144

Future studies . . . . . . . . . . . . . . . . . . . . . . 146

$\begin{array}{ll}\text { References } & 148\end{array}$ 


\section{List of Figures}

$1-1 \quad \mathrm{~N}_{2}$ fixation and new production $\ldots \ldots \ldots \ldots \ldots \ldots \ldots$

2-1 Dendrograms of previous classification of Trichodesmium . . . . . . . . 39

2-2 Typical absorption peaks . . . . . . . . . . . . . . . . . . . . . . 39

2-3 16S neighbor-joining tree $\ldots \ldots \ldots \ldots \ldots \ldots \ldots$

2-4 16S parsimony tree . . . . . . . . . . . . . . . . . . . 49

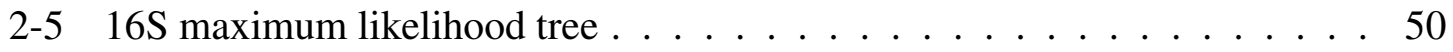

2-6 ITS neighbor-joining tree $\ldots \ldots \ldots \ldots \ldots \ldots \ldots \ldots$

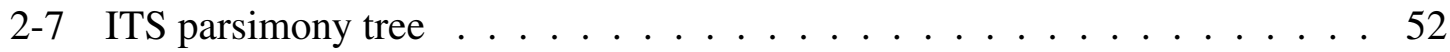

2-8 ITS maximum likelihood $\ldots \ldots \ldots \ldots \ldots \ldots \ldots$

2-9 het $R$ neighbor-joining tree $\ldots \ldots \ldots \ldots \ldots \ldots$

2-10 hetR parsimony tree . . . . . . . . . . . . . 55

2-11 hetR maximum likelihood tree . . . . . . . . . . . . . . . 56

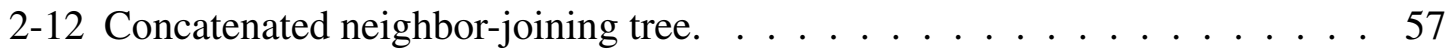

2-13 Concatenated parsimony tree. . . . . . . . . . . . . . 58

2-14 Concatenated maximum likelihood tree. . . . . . . . . . . . . . 59

2-15 Micrographs of Trichodesmium clade I . . . . . . . . . . . . . . 62

2-16 Micrographs of Trichodesmium clade II . . . . . . . . . . . . . . 64

2-17 Phycobiliprotein absorption spectra $\ldots \ldots \ldots \ldots 6$

3-1 Image processing for automated cell counts $\ldots \ldots \ldots \ldots$. . . . 77

3-2 Automated cell count method performance . . . . . . . . . . . . 80 
3-3 Standard curves . . . . . . . . . . . . . . . . . . 82

3-4 Melting curves $\ldots \ldots \ldots \ldots \ldots \ldots \ldots \ldots \ldots$

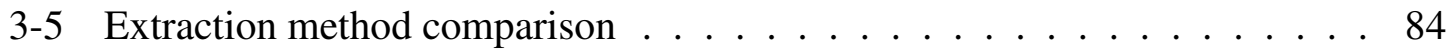

3-6 Serial dilutions of Trichodesmium cultures . . . . . . . . . . . . . 85

3-7 Mixed culture qPCR results . . . . . . . . . . . . . . . . . 85

4-1 Maps of cruise tracks . . . . . . . . . . . . . . . . . . . 92

4-2 Equatorial Atlantic data . . . . . . . . . . . . . . 96

4-3 West Pacific warm pool data . . . . . . . . . . . . . . 97

4-4 South Pacific data . . . . . . . . . . . . . . . . . . . . . 98

4-5 qPCR results versus cell counts . . . . . . . . . . . . . . . . . 99

4-6 Clade II versus clade I . . . . . . . . . . . . . . . . . . . . . . . . . . 99

4-7 Fe, P, and Trichodesmium . . . . . . . . . . . . . . . . 100

4-8 $\quad \mathrm{Fe}, \mathrm{P}$, and clade II:clade I . . . . . . . . . . . . . . . . . . 101

4-9 Depth, temperature, and Trichodesmium . . . . . . . . . . . . . 102

4-10 Temperature and depth histograms . . . . . . . . . . . . 103

4-11 Depth, temperature, and clade II:clade I . . . . . . . . . . . . . . 104

4-12 Depth of maximum concentration and mixed layer depth . . . . . . 105

4-13 Depth of maximum concentration histograms . . . . . . . . . . 106

4-14 Melt curves . . . . . . . . . . . . . . . . . . . . 107

4-15 qPCR product parsimony tree . . . . . . . . . . . . . . 108

4-16 Histogram of temperature and depth for clade I qPCR products . . . . . . 109

4-17 Diel cycle for the west Pacific warm pool . . . . . . . . . . . 111

5-1 Maps of cruise tracks . . . . . . . . . . . . . . . . . . 124

5-2 Hydrographic conditions of KM0701 . . . . . . . . . . . . . . . 127

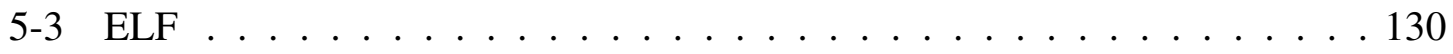

5-4 Organisms associated with Trichodesmium colonies . . . . . . . . . 131

$5-5$ DIP histogram . . . . . . . . . . . . . . . . . . 132 
5-6 Nitrogenase activity bar plot . . . . . . . . . . . . . 135 


\section{List of Tables}

1.1 Physical characteristics of Trichodesmium spp. . . . . . . . . . . . . . 24

1.2 Distribution of Trichodesmium spp. . . . . . . . . . . . . . 25

2.1 Species names . . . . . . . . . . . . . . . . . . 38

2.2 Culture collection . . . . . . . . . . . . . . . 41

2.3 Summary of PCR primers. . . . . . . . . . . . . . . . . 44

2.4 Morphology summary ...................... 61

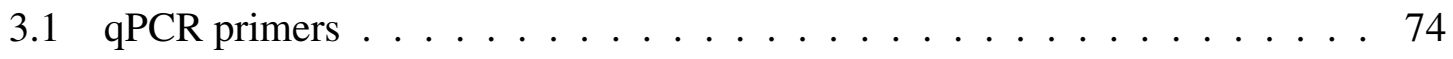

5.1 ELF data summary . . . . . . . . . . . . . . . . . . . 129

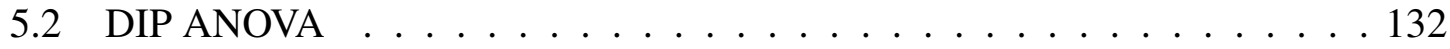

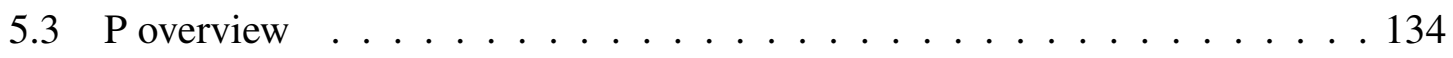




\section{CHAPTER 1}

\section{Introduction}

\section{Statement of Purpose}

Trichodesmium is a colonial, $\mathrm{N}_{2}$-fixing cyanobacterium and a significant source of fixed nitrogen $(\mathrm{N})$ in oligotrophic oceans. Its roles in the biogeochemical cycling of $\mathrm{N}$, phosphorus $(\mathrm{P})$, iron $(\mathrm{Fe})$, and carbon $(\mathrm{C})$ have made it the target of a wide variety of physiological, ecological, optical, and modeling studies. Trichodesmium spp. form colonies visible to the naked eye, making field collections and observations easier than for many other phytoplankton. There is currently a genome available for Trichodesmium erythraeum IMS101, aiding genetic studies of this genus. Teasing out the factors that may lead to niche differentiation in Trichodesmium addresses Hutchinson's "paradox of the plankton," whereby many more species of plankton coexist than would be expected based on the number of limiting resources available (Hutchinson, 1961). Lessons learned from Trichodesmium can be applied to other species of phytoplankton which are not as wellstudied.

There are several morphologically distinct but genetically similar species of Trichodesmium that coexist in tropical and subtropical waters. I hypothesized that the coexistence of several Trichodesmium species is due in part to niche differentiation. Trichodesmium spp. occupy different niche spaces to utilize resources such as nutrients and light and to respond to physical characteristics such as temperature and turbulence. To 
explore the niche spaces occupied by Trichodesmium spp., I investigated cultured strains available in the Woods Hole culture collection and natural communities in the Atlantic and Pacific Oceans. I characterized cultured Trichodesmium strains by sequencing the heterocyst differentiation gene hetR, 16S rDNA, and the internal transcribed spacer (ITS) regions; photographing and measuring trichomes (filaments); and analyzing the absorption spectra of phycobiliproteins (PBP). I used the hetR sequences to develop a real-time quantitative polymerase chain reaction (qPCR) method to identify and quantify the two major clades of Trichodesmium in cultures and field samples. I applied this qPCR method to resolve spatial distributions of Trichodesmium from samples collected with Niskin bottles across the Equatorial Atlantic, the west Pacific warm pool, and the South Pacific. Phosphorus stress was assayed using enzyme-labeled fluorescence (ELF) of alkaline phosphatase (AP) and $\mathrm{N}_{2}$ fixation was measured using acetylene reduction in Trichodesmium populations across the west Pacific warm pool and the western North Atlantic.

\section{Ecological roles of Trichodesmium}

Cyanobacteria evolved $3500 \mathrm{Ma}$ ago and dramatically changed the redox state of the Earth's atmosphere through oxygenic photosynthesis (Schopf, 2000). Also known as blue-green algae, cyanobacteria are found in both fresh and marine waters and a wide variety of terrestrial habitats. They are ubiquitous, prone to blooms, and can produce toxins (Cohen and Gurevitz, 2006). Trichodesmium spp. are filamentous, colonial, nonheterocystous, $\mathrm{N}_{2}$-fixing cyanobacteria belonging to the order Oscillatoriales (Waterbury, 2006). The genus name comes from the Greek root "trikh-" meaning "hair", referring to the filaments known as trichomes. Trichodesmium is found in tropical and subtropical oligotrophic waters with shallow mixed layer depths and warm temperatures.

Some cyanobacteria such as Trichodesmium are diazotrophs and contribute to new $\mathrm{N}$ inputs in oligotrophic systems (Capone et al., 1997). Fixed $\mathrm{N}$ is considered to be the proximal limiting nutrient in ocean ecosystems. For a system in steady-state, new production is stimulated by inputs of new $\mathrm{N}$, resulting in excess production available for export 


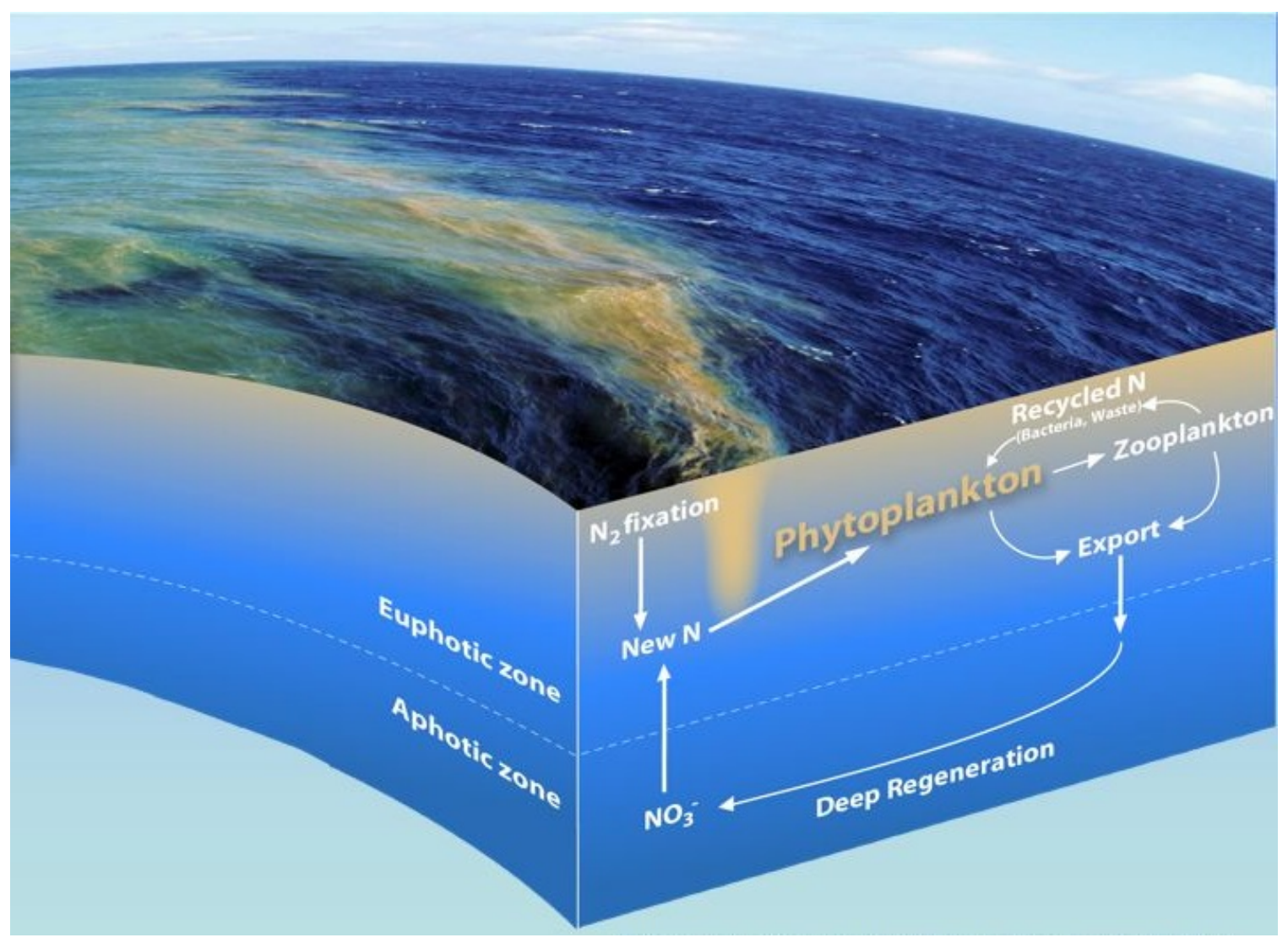

Figure 1-1: Trichodesmium and other diazotrophs fuel new production through $\mathrm{N}_{2}$ fixation. Photo shows a surface slick of Trichodesmium in the western South Pacific Ocean. Inputs of new nitrogen stimulate the growth of phytoplankton. New production can be exported out of the euphotic zone, drawing down surface concentrations of carbon. Photo courtesy of Daniel Ohnemus. Illustration by E. Paul Oberlander, WHOI.

(Fig. 1-1) (Eppley and Peterson, 1979). New N comes from eddy diffusion processes, seasonal deep mixing, atmospheric deposition, lateral advection, and $\mathrm{N}_{2}$ fixation (Karl, 2002). In the North Atlantic, $\mathrm{N}_{2}$ fixation rates of Trichodesmium can equal or exceed the vertical flux of $\mathrm{NO}_{3}^{-}$into surface waters (Capone et al., 2005), while in the North Pacific, $\mathrm{N}_{2}$ fixation is the source of up to half of the new $\mathrm{N}$ (Karl et al., 1997).

Trichodesmium is also a significant contributor of fixed C, through its own photosynthesis and through stimulation of primary production of other organisms (Fig. 1-1). Trichodesmium can account for 8-47\% of total primary production in the tropical North Atlantic (Carpenter et al., 2004). In a global marine ecosystem model, the presence of 
$\mathrm{N}_{2}$-fixers increased primary production by diatoms and small phytoplankton through the excretion of dissolved organic nitrogen (DON) in N-limited areas (Moore et al., 2002; Moore and Doney, 2007). Model results by Coles et al. (2004) showed that $\mathrm{N}_{2}$ fixation increases dissolved inorganic nirogen (DIN), phytoplankton biomass, primary production and export flux in the subtropics.

Factors affecting the growth of Trichodesmium include nutrients such as $\mathrm{P}$ and Fe, temperature, light intensity, mixed layer depth (MLD), and turbulence (Capone et al., 1997; Post, 2005). The amelioration of $\mathrm{N}$ limitation through $\mathrm{N}_{2}$ fixation can cause a drawdown of other nutrients such as $\mathrm{P}$ and Fe (Karl, 2002). The North Atlantic receives more dust input than the North Pacific, and the Fe available in terrestrial dust is necessary for the enzyme nitrogenase used in $\mathrm{N}_{2}$ fixation. Low excess $\mathrm{N}$ relative to excess $\mathrm{P}$ in dissolved nutrients is a necessary but not sufficient condition for selection for $\mathrm{N}_{2}$-fixing organisms in oligotrophic regions; in N-limited scenarios, dominant competitors such as diatoms cannot grow well and diazotrophs or diatoms with diazotrophic symbionts can succeed (Karl, 2002). When nutrient $\mathrm{N}: \mathrm{P}<16$, the classical Redfield ratio, diazotrophs are able to compete, drawing down $\mathrm{P}$ while increasing $\mathrm{N}$ and making the system more P-limited (Karl, 2002). Trichodesmium spp. have been shown to be able to use organic sources of P (Dyhrman et al., 2006; Sohm and Capone, 2006) and are capable of luxury uptake of Fe and P (Kustka et al., 2003; White et al., 2006a). In a numerical model simulating vertical migration of Trichodesmium, colonies $>1000 \mu \mathrm{m}$ were shown to be capable of P-mining: sinking by carbohydrate ballasting below the phosphocline, taking up P, and then rising back up to the euphotic zone (White et al., 2006b). Differential adaptations to nutrient limitation including alternative nutrient sources, uptake rates, halfsaturation constants, luxury uptake, and P-mining can lead to niche differentiation or coexistence of species limited by different resources (Titman, 1976).

In addition to its roles in $\mathrm{N}_{2}$ and $\mathrm{C}$ fixation, Trichodesmium provides substrate and shelter. A variety of organisms ranging from bacteria to crustacean larvae are enriched by 2-5 orders of magnitude in association with Trichodesmium colonies (Sheridan et al., 
2002). The copepod Macrosetella gracilis uses Trichodesmium as a substrate for juvenile development as well as a food source (O'Neil, 1998). Trichodesmium blooms can be undesirable in regions like the Gulf of Mexico, however, because they can produce toxins and contribute little to the marine food web, being grazed upon by only a few pelagic harpacticoid copepods: M. gracilis, Miracia efferata, and Oculosetella gracilis (O’Neil and Roman, 1994; Post, 2005).

\section{Species identifications and distributions}

There are six well-described species of Trichodesmium: T. erythraeum, T. thiebautii, Trichodesmium contortum, Trichodesmium tenue, Trichodesmium hildebrandtii and Trichodesmium pelagicum (formerly Katagnymene pelagica and Katagnymene spiralis). Traditionally, species of filamentous, non-heterocystous cyanobacteria such as Trichodesmium were identified using morphological characteristics including cell width, cell length, filament spirality, color, gas vacuoles, presence or absence of a sheath, and colony morphology (Table 1.1) (Janson et al., 1995; Orcutt et al., 2002; Waterbury, 2006). However, these characteristics can often prove to be problematic and many studies refer to field populations merely as Trichodesmium spp. or identify them by colony morphology: spherical "puffs," fusiform "rafts" or "tufts," and "bowties" (Janson et al., 1995). Morphological traits used to identify Trichodesmium spp. "correlate poorly" with genetic data, as shown by the clustering of the species formerly known as $K$. pelagica and $K$. spiralis among species of Trichodesmium (Lundgren et al., 2005). The different species of Trichodesmium are closely related. Sequences of the nitrogenase gene nifH, the heterocyst differentiation gene hetR, 16S rRNA, and the ITS region as well as fingerprinting of HIP1 show low genetic diversity among Trichodesmium (Ben-Porath et al., 1993; Orcutt et al., 2002; Lundgren et al., 2005). 
Table 1.1: Physical characteristics and colony morphologies of Trichodesmium spp.(Janson et al., 1995; Orcutt et al., 2002; Lundgren et al., 2005)

\begin{tabular}{lccccccl}
\hline Species & puff & tuft & bowtie & trichomes & $\begin{array}{c}\text { Cell width } \\
(\mu \mathrm{m})\end{array}$ & $\begin{array}{c}\text { Cell length } \\
(\mu \mathrm{m})\end{array}$ & Other \\
\hline T. aureum & & & & $\checkmark$ & $20-25$ & & gold \\
T. contortum & & & & $\checkmark$ & $30-40$ & & \\
T. erythraeum & & $\checkmark$ & & $\checkmark$ & $6-9$ & $4-10$ & red-brown \\
T. hildebrandtii & $\checkmark$ & & $\checkmark$ & $\checkmark$ & $14-17$ & $5-12$ & \\
T. pelagicum & & & & & & & straight trichome \\
$\quad$ K. pelagica & & & & $\checkmark$ & $15-35$ & & coiled trichome \\
$\quad$ K. spiralis & & & & $\checkmark$ & $12-23$ & $4-7$ & \\
T. tenue & & $\checkmark$ & $\checkmark$ & $\checkmark$ & $5-7$ & $10-19$ & \\
T. thiebautii & $\checkmark$ & $\checkmark$ & & $\checkmark$ & $7-9$ & $6-12$ & \\
\hline
\end{tabular}

The distribution of Trichodesmium is influenced by temperature. Temperature sets an overall upper limit on growth rate (Eppley, 1972). Increased temperature within a viable range will increase enzyme activity, hence increasing cellular functions such as respiration; photosynthesis, particularly the Calvin Cycle; and $\mathrm{N}_{2}$ fixation. In the eastern Atlantic, Trichodesmium is found at highest densities between $0-15^{\circ} \mathrm{N}$, with a complete absence south of $30^{\circ} \mathrm{S}$ (Tyrrell et al., 2003). Blooms are found in surface waters with temperatures $\geq 25^{\circ} \mathrm{C}$, but trichomes can be found in deeper waters with temperatures of $21-23^{\circ} \mathrm{C}(\mathrm{Karl}, 2002)$.

While often found concurrently, the species of Trichodesmium have different distributions. Table 1.2 gives an overview of the dominant species found in different ocean basins. While abundance varies in time and space, T. thiebautii is generally most common in the Northern Hemisphere while T. erythraeum is most common in the Great Barrier Reef (Table 1.2) (Orcutt and Gundersen, 2003; Bell et al., 2005). At the Bermuda Atlantic Time-series Station (BATS), T. thiebautii puffs are present year-round, but during the summer maximum of Trichodesmium abundance, T. thiebautii tufts are the predominant morphology, followed by T. thiebautii puffs and T. erythraeum tufts (Orcutt and Gundersen, 2003). In addition to temporal and horizontal spatial variation, colony morphologies show different vertical distributions. In the Gulf of Aqaba, Red Sea, tuft colonies were found near the surface while puff colonies were found in the lower half of the photic zone 
(Post et al., 2002). During a trans-Atlantic survey with a video plankton recorder (VPR), tufts were more abundant in the upper $50 \mathrm{~m}$ while puffs were abundant throughout the euphotic zone (Davis and McGillicuddy, 2006).

Table 1.2: Distribution of Trichodesmium spp.

\begin{tabular}{|c|c|c|c|c|}
\hline Region & $\begin{array}{l}\text { Dominant } \\
\text { Species }\end{array}$ & $\begin{array}{l}\text { Other Common } \\
\text { Species }\end{array}$ & Rare Species & Source \\
\hline N. Atlantic & T. thiebautii & T. erythraeum & & $1,2,3$ \\
\hline N. Pacific & $\begin{array}{l}\text { T. thiebautii, } \\
\text { T. erythraeum }\end{array}$ & T. contortum & & 4 \\
\hline $\begin{array}{l}\text { S. Pacific (Great Barrier } \\
\text { Reef) }\end{array}$ & T. erythraeum & & & 5 \\
\hline $\begin{array}{l}\text { S. Pacific (New Caledo- } \\
\text { nia) }\end{array}$ & $\begin{array}{l}\text { T. thiebautii, } \\
\text { T. tenue }\end{array}$ & T. erythraeum & & 6,7 \\
\hline S. Pacific (open ocean) & T. thiebautii & $\begin{array}{l}\text { T. erythraeum, } \\
\text { T. pelagicum }\end{array}$ & & 7 \\
\hline Red Sea & $\begin{array}{l}\text { T. thiebautii, } \\
\text { T. erythraeum }\end{array}$ & T. tепие & T. hildebrandtii & 8 \\
\hline Western Indian Ocean & T. erythraeum & $\begin{array}{l}\text { T. thiebautii, T. tenue, } \\
\text { Trichodesmium sp. }\end{array}$ & T. contortum & 9 \\
\hline
\end{tabular}

Within an ocean basin, there is high spatial and temporal variation in Trichodesmium densities (Carpenter et al., 2004; Capone et al., 2005). Mesoscale eddies can influence phytoplankton communities by upwelling or downwelling nutrients and affecting temperature. In the North Atlantic, diatoms and dinoflagellates bloom in upwelling mode-water eddies while cyanobacteria such as Synechococcus are prevalent in cyclones (Sweeney et al., 2003), and Trichodesmium are associated with warm, downwelling anti-cyclonic eddies (Davis and McGillicuddy, 2006). Trichodesmium are also associated with smaller physical features such as windrows and Langmuir cells (Carpenter and Price, 1977; Carpenter and Capone, 1992). Buoyant Trichodesmium colonies and trichomes accumulate at the surface in convergence zones of these features. 


\section{Competition theory}

According to resource competition theory, the species which is best able to utilize and deplete the limiting resource will exclude competing species in a steady-state environment (Titman, 1976). Species with different limiting resources could coexist (Tilman, 1977). An environment at steady-state is predicted to be able to hold only as many competing species as it has limiting resources. However, there are many more species of phytoplankton than there are limiting resources, resulting in Hutchinson's "plankton paradox" (Hutchinson, 1961). Processes which support coexistence of species, and hence address the plankton paradox, include spatial and temporal heterogeneity, differing mortality rates, and niche differentiation along physical gradients as well as resource gradients (Levins, 1979; Tilman et al., 1981).

Competition between two species can be illustrated with the Lotka-Volterra competition model:

$$
\begin{aligned}
\frac{1}{N_{1}} \frac{d N_{1}}{d t} & =\left(r_{1}-\alpha_{11} N_{1}-\alpha_{12} N_{2}\right) \\
\frac{1}{N_{2}} \frac{d N_{2}}{d t} & =\left(r_{2}-\alpha_{21} N_{1}-\alpha_{22} N_{2}\right),
\end{aligned}
$$

where $N_{i}$ is the population size of species $i, r_{i}$ is the maximal growth rate for species $i, \alpha_{i i}$ is the intraspecific competition coefficient $\left(\alpha_{i i}=\frac{1}{K_{i}}\right.$ where $K_{i}$ is the carrying capacity), and $\alpha_{i j}$ is the competition coefficient parameterizing the strength of effect of species $j$ on species $i$ (Kot, 2001). This model incorporates both inter- and intraspecific competition for resources. The success of one species over the other depends on the relative strengths of the competition coefficients $\alpha_{i j}$. Species 1 wins if $\frac{r_{1}}{\alpha_{11}}<\frac{r_{2}}{\alpha_{21}}$ and $\frac{r_{2}}{\alpha_{22}}>\frac{r_{1}}{\alpha_{12}}$. In this case, intraspecific competition of species 1 is relatively greater than the competitive effect of species 2 and the effect of species 1 on 2 is greater than intraspecific competition of species 2. Likewise, species 2 wins if $\frac{r_{1}}{\alpha_{11}}>\frac{r_{2}}{\alpha_{21}}$ and $\frac{r_{2}}{\alpha_{22}}<\frac{r_{1}}{\alpha_{12}}$. Coexistence occurs if $\frac{r_{1}}{\alpha_{11}}<\frac{r_{2}}{\alpha_{21}}$ and $\frac{r_{2}}{\alpha_{22}}<\frac{r_{1}}{\alpha_{12}}$, if intraspecific competition is greater than interspecific competi- 
tion. Either species dominates, depending on initial conditions if $\frac{r_{1}}{\alpha_{11}}>\frac{r_{2}}{\alpha_{21}}$ and $\frac{r_{2}}{\alpha_{22}}>\frac{r_{1}}{\alpha_{12}}$ (Shigesada and Kawasaki, 1997). The Lotka-Volterra model is descriptive and assumes an environment in equilibrium where $r_{i}$ and $\alpha_{i j}$ are fixed (Tilman et al., 1981). However, in dynamic environments such as the open ocean, coexistence may occur. Trichodesmium spp. $r_{i}$ and $\alpha_{i j}$ may vary depending on the physical and nutrient environments.

A more mechanistic model of competition uses the Monod equation, similar to Michaelis-Menton enzyme kinetics. Monod's equation relates the growth rate of a population to the amount of resource available in a continuous flow environment:

$$
\begin{aligned}
\frac{1}{N_{i}} \frac{d N_{i}}{d t} & =\min _{j}\left(\frac{r_{i} S_{j}}{k_{i j}+S_{j}}-D\right) \\
\frac{d S_{j}}{d t} & =D\left(S_{j 0}-S_{j}\right)-\sum_{i=1}^{n} \frac{N_{i} r_{i} S_{j}}{\left(k_{i j}+S_{j}\right) Y_{i j}}
\end{aligned}
$$

where $S_{j}$ is the external concentration of resource $j, k_{i j}$ is the half saturation constant for species $i$ limited by resource $j, D$ is the dilution rate for a continuous flow system and the steady-state growth rate, $S_{j 0}$ is the influent concentration of resource $j, n$ is the number of species, and $Y_{i j}$ is the yield of species $i$ limited by resource $j$ measured in cells per resource (Tilman, 1977). The growth of species $i$ is limited by the resource which gives the smallest per capita growth rate. The steady-state solution to the Monod model is analogous to the steady-state form of the Lotka-Volterra model, but the Monod model allows explicit examination of an environment which is not in steady-state (Tilman, 1977). Coexistence is possible if each species is limited by a different resource and two species limited by the same resource can coexist if $\frac{k_{11}}{r_{1}-D}=\frac{k_{21}}{r_{2}-D}$ (Tilman, 1977). For example, if one species of Trichodesmium is more limited by $\mathrm{P}$ while the other is more limited by light or Fe, they may be able to coexist.

A third model of resource competition is a variable internal stores model (Droop, 1974). This model also follows Michaelis-Menten kinetics but relates growth to internal 
stores of resources, or cell quotas:

$$
\begin{aligned}
\frac{1}{N_{i}} \frac{d N_{i}}{d t} & =\min _{j}\left(\frac{r_{i}\left(1-g_{i j}\right)}{Q_{i j}}-D\right) \\
\frac{d Q_{i j}}{d t} & =V_{i j}\left(\frac{S_{j}}{k_{i j}+S_{j}}\right)-r_{i}\left(Q_{i j}-g_{i j}\right), \\
\frac{d S_{j}}{d t} & =D\left(S_{j 0}-S_{j}\right)-\sum_{i=1}^{n} N_{i} V_{i j} \frac{S_{j}}{k_{i j}+S_{j}},
\end{aligned}
$$

where $q_{i j}$ is the minimum internal store of resource $\mathrm{j}$ by species $\mathrm{i}, Q_{i j}$ is the cell quotient of resource $\mathrm{j}$ in species $\mathrm{i}$, and $V_{i j}$ is the maximal uptake rate of resource $\mathrm{j}$ by species $\mathrm{i}$ (Tilman, 1977). The internal stores model is more complicated than the Monod model because it allows for luxury uptake of nutrients by phytoplankton. At equilibrium, the two models are equivalent, and although they showed different behavior under non-equilibrium conditions, the superior competitor in the Monod model was generally the superior competitor in the variable internal stores model (Grover, 1990). Variable stores predicts three outcomes depending on the flow rate $D$ and ratio of the two nutrients: both species limited by nutrient 1 , both species limited by nutrient 2 , or coexistence.

The above models examine up to two limiting resources. When models include three or more limiting resources, oscillations and chaos can result in a monod-type model (Huisman and Weissing, 1999). The internal dynamics are complex enough to generate non-equilibrium conditions, and several competing species can persist on just a few resources (Huisman and Weissing, 1999). A variable internal stores model also had oscillations and chaos that allowed more species than resources to coexist, and the oscillations were damped when compared to those of a Monod model (Revilla and Weissing, 2008). These chaotic oscillations have also been observed in long-term culture experiments with multiple trophic levels and constant physical conditions (Benincà et al., 2008). Physiology and life history also play a role in competition dynamics; incorporation of a trade-off between competitive abilities and a cyclic relation between competitive ability and resource content into a competiton model with three resources generated oscillations which 
enabled more than 100 species to coexist (Huisman et al., 2001). When resource limitation is combined with physical factors such as temperature, $\mathrm{pH}$, or salinity, competitive advantages can shift from one species to another (Tilman et al., 1981). Trichodesmium spp. compete with each other and with other phytoplankton for nutrients such as $\mathrm{P}$ and $\mathrm{Fe}$ and for light. The effects of pigmentation, temperature, and salinity can affect the kinetics of nutrient uptake as well as rates of $\mathrm{N}_{2}$ fixation, potentially differentiating niches for Trichodesmium.

In addition to the internal nonequilibrium dynamics of a competitive system, the ocean itself is rarely, if ever, in steady-state when considering the space and time scales of a phytoplankter. Phytoplankton are distributed discretely, so mass conservation models such as resource-based competition models may not always be valid (Siegel, 1998). Rates of competition increase with cell size and abundance, so in the oligotrophic ocean where abundance is low, competitive exclusion may take so many generations that other factors such as nutrient pulses or changes in physical properties become more important in population dynamics than competition (Siegel, 1998). Stochastic perturbations (noise) from the environment can cause shifts in model dynamics and decrease the predictability of a system (Bailey et al., 2004). Episodic events such as dust deposition, mesoscale eddies, and turbulent mixing can affect community dynamics of Trichodesmium. The spatial and temporal heterogeneity of the ocean as well as relevant scales must be considered when modeling competition.

\section{Niche differentiation in cyanobacteria}

A niche is the combination of the actions and relationships of an organism in its community (Hutchinson, 1957). In classical niche theory, overlapping niches compete strongly with each other and differentiation enables coexistence. Genomic and culture studies of unicellular cyanobacteria have shown evidence of niche differentiation. Two closelyrelated Synechococcus isolates from the Baltic Sea have different pigmentation, red and blue-green, which allow them to coexist by partitioning the light spectrum (Stomp et al., 
2004). Prochlorococcus has a high light adapted strain (MED4) that uses $\mathrm{NH}_{4}^{+}$as a $\mathrm{N}$ source and a low light adapted strain (MIT9313) that uses $\mathrm{NH}_{4}^{+}$and $\mathrm{NO}_{2}^{-}$as $\mathrm{N}$ sources (Rocap et al., 2003). Uncultured strains of Prochlorococcus in the North Pacific have been found to have assimilation genes for both $\mathrm{NO}_{3}^{-}$and $\mathrm{NO}_{2}^{-}$in both high and low light adapted ecotypes, so $\mathrm{N}$ sources do not directly correspond to light adaptation (Martiny et al., 2009). Differences in the vertical distribution of Prochlorococcus ecotypes were found between the western and eastern North Atlantic using qPCR, probe hybridization, and flow cytometry (Zinser et al., 2006). A north-south transect of the Altantic Ocean showed distributions of ecotypes of Prochlorococcus following patterns of temperature, light, and nutrients (Johnson et al., 2006). These biogeographical patterns have emerged in an ecosystem model of multiple functional groups with randomly generated parameters, including Prochlorococcus-like functional groups (Follows et al., 2007).

The ecology of field populations of Trichodesmium varies according to colony morphology and species. Puffs and tufts have different depth distributions (Post et al., 2002; Davis and McGillicuddy, 2006). Trichodesmium colony morphologies also respond differentially to physical forcing; at BATS, T. thiebautii puffs rather than tufts become the predominant form in surface waters when the sea surface temperature is greater than $26^{\circ} \mathrm{C}$ and winds maintain a turbulent mixed layer (Orcutt and Gundersen, 2003). Puffs, tufts and bowties also show varying activities in enzymes such as alkaline phosphatase, an enzyme which can cleave phosphate from organic phosphate and is expressed under P stress (Stihl et al., 2001; Webb et al., 2007; Hynes et al., 2009). $\mathrm{N}_{2}$ fixation rates can differ significantly between colony types locally (Webb et al., 2007). Grazing rates by copepods on Trichodesmium vary with species and colony morphology, presumably due to presence of a neurotoxin; M. gracilis and M. efferata had higher ingestion rates on T. erythraeum than on T. thiebautii and higher ingestion rates on puffs than tufts of T. thiebautii (O'Neil and Roman, 1994).

Cyanobacteria have chlorophyll $a(\mathrm{Chl} a)$ as the primary photosynthetic pigment with phycobiliproteins (PBP) including phycoerythrin (PE), phycocyanin (PC), and al- 
lophycocyanon (AP) as accessory light harvesting pigments (Waterbury et al., 1986). Species of Trichodesmium have different pigmentation and their colors range from dark red-brown (T. erythraeum IMS101) to light salmon (T. tenue tenue) to light green (Trichodesmium sp. H9-4). T. erythraeum has more PE than T. thiebautii (Carpenter et al., 1993). These observations of variable characteristics across colony morphologies and species of Trichodesmium point to possible niche differentiation.

\section{Overview of thesis chapters}

Trichodesmium contributes a significant amount of fixed $\mathrm{N}$ to marine ecosystems (Capone et al., 1997), and measurements of Trichodesmium have been used to parameterize $\mathrm{N}_{2}$ fixation in a variety of models (Moore et al., 2004; Coles et al., 2004). However, much is

unknown about its ecology and physiology. Mortality rates and causes of mortality are not well characterized, distributions are patchy, and potential differences among species of Trichodesmium have not been thoroughly studied and are often ignored. A vast majority of the culture experiments have used T. erythraeum IMS101, which belongs to a clade that branches separately from the numerically predominant species of T. thiebautii (Chaps. $2,3)$. This species is also used to parameterize $\mathrm{N}_{2}$ fixation in global ecosystem models (Moore et al., 2004). To understand the roles Trichodesmium plays in the ecosystem and the factors that control its distribution, it is important to understand the differences among the species of Trichodesmium and the potential for niche differentiation. I aimed to answer the questions:

- What species of Trichodesmium do we have in the Woods Hole culture collection?

- How are these cultured strains related to each other?

- Do the cultured strains have characteristics which might lead to niche differentiation?

- How are field populations of Trichodesmium distributed?

- What factors control the distributions of Trichodesmium spp.? 
In my PhD thesis, I characterized cultured strains of Trichodesmium, developed a molecular method to distinguish between the two clades of Trichodesmium, identified and enumerated Trichodesmium clades in the field, and investigated $\mathrm{P}$ stress and $\mathrm{N}_{2}$ fixation in field populations.

Chapter 2: Characterization. The Woods Hole Oceanographic Institution has an extensive culture collection of Trichodesmium spp., established and maintained over fifteen years through the work of Dr. John Waterbury. This collection gave me the unique opportunity to take measurements from a variety of isolates. The genome of T. erythraeum IMS101 is currently available, allowing for the development of primers to target genetic markers for identification such as hetR, 16S rDNA and ITS. Species of Trichodesmium vary by cell length, cell width, and colony morphology. Using phase contrast light microscopy and differential interference contrast (DIC) microscopy, I measured cell width and length and observed gas vesicles of cultured strains. I analyzed the PBP absorption spectra for the cultured strains of Trichodesmium and measured PUB:PEB and PE:PC ratios by the relative peak heights. I found that the cultured species of Trichodesmium clustered into two clades: one containing T. erythraeum and T. contortum, the other containing T. thiebautii, T. tenue, T. pelagicum, and T. hildebrandtii. Each clade has diverse cell morphology, but distinct PBP absorption spectra.

Chapter 3: qPCR. The hetR gene is putatively used in cyanobacterial heterocyst differentiation and may be used in diazocyte differentiation in Trichodesmium (ElShehawy et al., 2003). Using hetR sequences from Lundgren et al. (2005) and cultured strains (Chapt. 2), I developed clade-specific primers for approximately $140 \mathrm{bp}$ regions targeted for qPCR. The qPCR assay was optimized using known concentrations of cultured strains and standardized with cloned PCR products from each clade. I also tested a variety of DNA extraction methods to minimize PCR inhibition.

Chapter 4: Quantification of field populations. I quantified field samples of Trichodesmium from depth profiles along horizontal gradients of the equatorial Atlantic Ocean, the west Pacific warm pool, and the South Pacific Ocean. I collected water at var- 
ious depths including the surface and deep chlorophyll maximum (DCM) using a rosette of Niskin bottles. Trichodesmium clade I and II abundances and distributions were analyzed with ancillary data such as temperature, salinity, depth, and nutrient concentrations. Clade II was more numerous than clade I in both the Pacific and Atlantic Oceans. Clade I had deeper and colder distributions than clade II. There was a correlation between clade I and clade II concentrations in the Pacific but not the Atlantic. There were no patterns or Trichodesmium concentrations with respect to $\mathrm{P}$ or Fe.

Chapter 5: Phosphorus stress and $\mathbf{N}_{2}$ fixation. Phosphorus (P) can limit growth and $\mathrm{N}_{2}$ fixation rates of Tricodesmium. Under P stress, some phytoplankton use alkaline phosphatase (AP) to cleave phosphate from organic sources (Stihl et al., 2001). I analyzed $\mathrm{P}$ stress and $\mathrm{N}_{2}$ fixation rates of Trichodesmium populations from the Atlantic and $\mathrm{Pa}-$ cific Oceans. I collected colonies of Trichodesmium and analyzed them for endogenous AP activity using enzyme-labeled fluorescence (ELF) and for nitrogenase activity using acetylene reduction. Both $\mathrm{P}$ stress and $\mathrm{N}_{2}$ fixation rates were higher in the western North Atlantic than in the Pacific, indicating that factors other than $\mathrm{P}$ were constraining $\mathrm{N}_{2}$ fixation rates of Trichodesmium in the Pacific. 


\title{
CHAPTER 2
}

\section{Diversity of the $\mathbf{N}_{2}$-fixing Cyanobacterium}

\section{Trichodesmium: Characterization of the Woods Hole Culture Collection}

\begin{abstract}
:
The filamentous, colonial cyanobacterium Trichodesmium has six well-described species, but many more names. Traditional classification was based on morphological characteristics such as cell width and length, gas vesicle distribution, and colony morphology, which can lead to inconsistent results. I used the Woods Hole culture collection of Trichodesmium to identify cultured strains by species using cell morphology; phycobiliprotein absorption spectra; and sequences of $16 \mathrm{~S}$ rDNA, the 16S-23S internal transcribed spacer (ITS), and the heterocyst differentiation gene hetR. There were two major clades of Trichodesmium: clade I consisting of Trichodesmium erythraeum and Trichodesmium contortum, and clade II made up of Trichodesmium thiebautii, Trichodesmium tenue, Trichodesmium pelagicum, and Trichodesmium hildebrandtii. These clades were genetically coherent with similar phycobiliprotein composition, but morphologically diverse. In the continual revision of cyanobacterial taxonomy, genetic and biochemical information are useful and informative complements to morphology for informing classification schemes.
\end{abstract}




\section{Introduction}

Identification of Trichodesmium. Trichodesmium spp. (Cyanobacteriales, order Oscillatoriales) live in tropical and subtropical oceans. This filamentous phytoplankter exists as both single trichomes and colonies that are visible to the naked eye, earning the nickname "sea sawdust" (Cook, 1842). They occasionally form brown or red blooms that are visible from space and gave the Red Sea its name (Ehrenberg, 1830). In addition to contributing fixed carbon (C) to the ecosystem, Trichodesmium is a significant contributor of new nitrogen $(\mathrm{N})$ through $\mathrm{N}_{2}$ fixation in oligotrophic regions (Capone et al., 1997). While only a few grazers feed on Trichodesmium (O’Neil and Roman, 1994), colonies offer habitat by providing an oasis in the open ocean, harboring a community of heterotrophic bacteria and invertebrates (Sheridan et al., 2002; Hewson et al., 2009).

Classical identification of Trichodesmium was based on cell width and length, sheath characteristics, distribution of gas vesicles, and colony morphology. There are six well-described species of Trichodesmium: Trichodesmium contortum, Trichodesmium erythraeum, Trichodesmium hildebrandtii, Trichodesmium pelagicum (formerly Katagnymene spiralis and Katagnymene pelagica), Trichodesmium tenue, and Trichodesmium thiebautii. These species of Trichodesmium are genetically similar, but morphologically distinct. Classification by cell and colony morphologies can be confusing and misleading due to variable and overlapping characteristics (Anagnostidis and Komárek, 1988; Janson et al., 1995). Many genera in Oscillatoriaceae were identified by characteristics of the sheath, which can vary with environmental conditions and proved to be an unreliable criterion (Rippka et al., 1979; Hoffmann, 1988; Anagnostidis and Komárek, 1988). Trichodesmium colonies come in a variety of morphologies including spherical puffs, fusiform tufts, and bowties. Some species can have more than one morphology, for example, T. thiebautii forms both puff and tuft colonies, and different species can have the same colony morphology: T. thiebautii, T. hildebrandtii, and T. erythraeum all form tufts. Single colonies may also contain a variety of filaments, indicating that they are not 
clonal and suggesting that colonies may coalesce from single trichomes of several species (Hynes et al., 2009).

Even more perplexing is the list of names for species currently or formerly known as "Trichodesmium." Many species originally identified as Trichodesmium were brought under the umbrella of the genus Oscillatoria and then later were separated back to Trichodesmium (Geitler, 1932; Rippka et al., 1979; Anagnostidis and Komárek, 1988). Species of the genus Katagnymene were found to be genetically similar to T. thiebautii with respect to the nitrogenase gene nifH, the $16 \mathrm{~S}-23 \mathrm{~S}$ internal transcribed spacer (ITS), and the heterocyst differentiation gene hetR, so Katagnymene spp. have been included in the genus Trichodesmium (Lundgren et al., 2001; Orcutt et al., 2002; Lundgren et al., 2005). Due to the inconsistent nature of cell morphology, a large number of synonyms have been in use (Guiry and Guiry, 2008). A selection of synonyms is summarized in Table 2.1.

Previous studies have grouped Trichodesmium spp. into a variety of taxonomic schemes. The classification of species based on morphometric characteristics and cell structure resulted in two groups (Fig. 2-1A): (1) species with peripheral gas vesicles (T. tenue, a spherical-shaped colony from an undetermined Trichodesmium sp., and T. erythraeum); and (2) species with randomly dispersed gas vesicles (T. thiebautii, T. hildebrandtii, and T. contortum) (Janson et al., 1995). In phylogenetic trees of Trichodesmium, T. erythraeum branched off from all the other groups (Orcutt et al., 2002). Trees constructed from $16 \mathrm{~S}$ rRNA and hetR sequences of field and culture samples have shown four clades: (1) T. hildebrandii, T. thiebautii, and T. pelagicum; (2) a novel morphotype named Trichodesmium aureum; (3) T. erythraeum and a large, dark-pigmented trichome; and (4) T. contortum and T. tenue (Lundgren et al., 2005). This grouping is represented in the dendrogram in Fig. 2-1B.

Photosynthetic pigments. Members of the genus Trichodesmium have a photosynthetic system typical of other cyanobacteria. Their primary photosynthetic pigment is chlorophyll $a(\mathrm{Chl} a)$ with a light-harvesting phycobilisome made up of phycobiliproteins 
Table 2.1: List of species names for Trichodesmium, taken partly from Guiry and Guiry (2008).

\begin{tabular}{|c|c|c|}
\hline Species & Synonyms & Ref. \\
\hline Trichodesmium aureum & & 1 \\
\hline Trichodesmium clevei & Pelagothrix clevei J. Schmidt & 2,3 \\
\hline \multirow{2}{*}{ *Trichodesmium contortum } & Xanthotrichum contortum & 4 \\
\hline & Skujaella contorta & 5 \\
\hline \multirow[t]{4}{*}{ *Trichodesmium erythraeum } & Oscillaria erythraea (Ehrenberg) & 6 \\
\hline & Oscillatoria erythraea (Ehrenberg) & 7 \\
\hline & Trichodesmium ehrenbergii Montagne & 5 \\
\hline & Trichodesmium erythraeum Ehrenberg & 8 \\
\hline Trichodesmium flosaquae & & 5 \\
\hline Trichodesmium havanum & & 1 \\
\hline \multirow[t]{5}{*}{ *Trichodesmium hildebrandtii } & Oscillatoria hildenbrandtii & 7 \\
\hline & Skujaella hildebrandtii & 5 \\
\hline & Trichodesmium ehrenbergiif. indicum & 2 \\
\hline & Trichodesmium hildenbrandtii & 9 \\
\hline & Trichodesmium indicum & 5 \\
\hline Trichodesmium hindsii & & 7 \\
\hline Trichodesmium iwanoffianum Nygaard & Oscillatoria lacustris & 2 \\
\hline \multirow[t]{2}{*}{ Trichodesmium lacustre } & Oscillatoria lacustris & 2 \\
\hline & Skujaella lacustris & 5 \\
\hline Trichodesmium lenticulare & Haliarachne lenticularis & 7 \\
\hline Trichodesmium maccii & & 10 \\
\hline \multirow[t]{3}{*}{ *Trichodesmium pelagicum } & Katagnymene pelagica & 1,7 \\
\hline & Katagnymene spiralis & 7 \\
\hline & Trichodesmium spiralis & 11 \\
\hline Trichodesmium scoboideum & Skujaella scoboidea & 5 \\
\hline \multirow[t]{2}{*}{ *Trichodesmium tenue } & Trichodesmium radians & 2 \\
\hline & Heliotrichum radians & 12 \\
\hline \multirow[t]{3}{*}{ *Trichodesmium thiebautii } & Oscillatoria thiebautii & 7 \\
\hline & Skujaella thiebautii & 5 \\
\hline & Heliotrichum radians & 13 \\
\hline
\end{tabular}

*Species represented in the Woods Hole culture collection (past and present).

1 = Lundgren et al. (2005), 2 = Anagnostidis and Komárek (1988), 3 = Silva et al. (1996), $4=$ Schütt (1893), 5 = Drouet (1968), $6=$ Kützing (1845), $7=$ Geitler (1932), $8=$ Hallegraeff et al. (2003), $9=$ Gomont (1892), $10=$ Li et al. (1984), $11=$ Dyhrman et al. (2006), $12=$ Golubic (1977), $13=$ Wille (1904) 

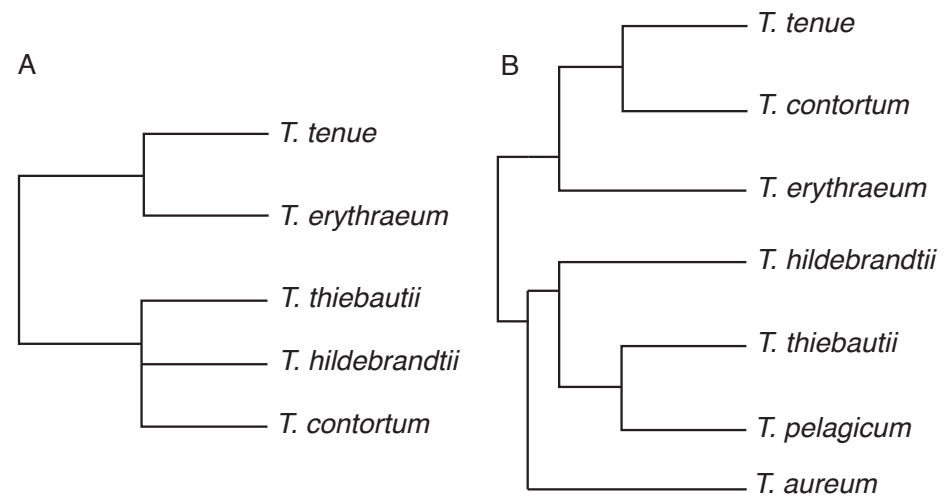

Figure 2-1: Dendrograms of classification groupings of Trichodesmium in previous studies. (A) Grouping based on morphological characteristics (Janson et al., 1995). (B) Grouping based on hetR sequences (Lundgren et al., 2005).

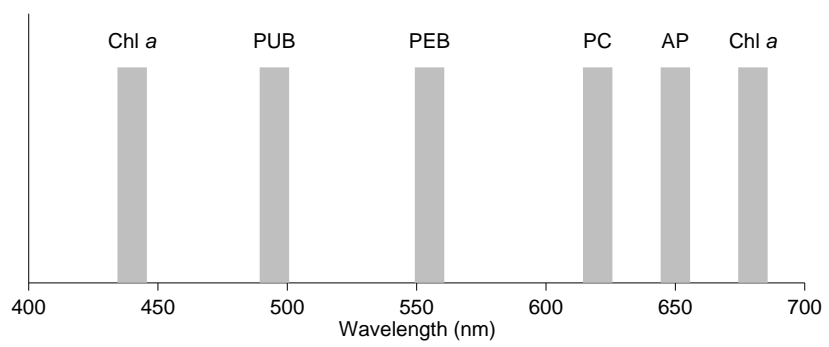

Figure 2-2: Typical absorption peak values for photosynthetic pigments in cyanobacteria: chlorophyll $a(\mathrm{Chl} a)$, phycourobilin (PUB), phycoerythrobilin (PEB), phycocyanin (PC), allophycocyanin (AP) (Rippka et al., 1974).

(Glazer, 1987). Trichodesmium phycobilisomes are dominated by the phycobiliprotein phycoerythrin (PE), which has absorbance peaks at 490 - $500 \mathrm{~nm}$ for its brown phycourobilin (PUB) component and at 545 - $565 \mathrm{~nm}$ for its red phycoerythrobilin (PEB) component. The phycobilisome also contains the blue-green phycocyanin (PC), which absorbs around $620 \mathrm{~nm}$, and the blue allophycocyanin (AP), which absorbs at $650 \mathrm{~nm}$ (Fig. 2-2). In contrast, Chla has absorption peaks at about $440 \mathrm{~nm}$ and $680 \mathrm{~nm}$ (Rippka et al., 1974).

Chromatic adaptation has been described in field populations and cultured Trichodesmium (Subramaniam et al., 1999; Bell and Fu, 2005). PUB:PEB ratios in Trichodesmium have been shown to increase at midday in the Caribbean Sea (Subrama- 
niam et al., 1999). Great Barrier Reef strain T. erythraeum GBRTRLI101 showed chromatic adaptation by changing its $\mathrm{PE}: \mathrm{Chl} a$ ratio as well as its $\mathrm{PE}: \mathrm{PC}$ ratio; $\mathrm{PE}$ increased under green light, PC increased under red light, and Chla increased under blue and red light (Bell and Fu, 2005). However, chromatic adaptation has not been found in other field studies (McCarthy and Carpenter, 1979; Neveux et al., 2006). Pigmentation of Trichodesmium in the field and in culture varies considerably with colors ranging from red to brown to green. This color variation is partly due to phycobiliprotein composition; Carpenter et al. (1993) noted that T. erythraeum had more phycoerythrin per colony and a higher PE:Chla than T. thiebautii. Color may also be influenced by growth state; senescent Trichodesmium cells appear more brown or green than healthy cells due to chlorosis.

Characterization of the Woods Hole culture collection. The Woods Hole Oceanographic Institution (WHOI) has an extensive collection of Trichodesmium cultures from all over the world (Table 2.2). To classify these strains and understand the relationships among the various species, I characterized the culture collection using cell morphology, phycobiliprotein spectra, and sequences of $16 \mathrm{~S}$ rDNA, ITS, and hetR. The results of this study showed that there are two major clades of Trichodesmium based on sequence analysis. Surprisingly, the clades were not morphologically homogeneous, but were consistent with respect to phycobiliprotein content. 


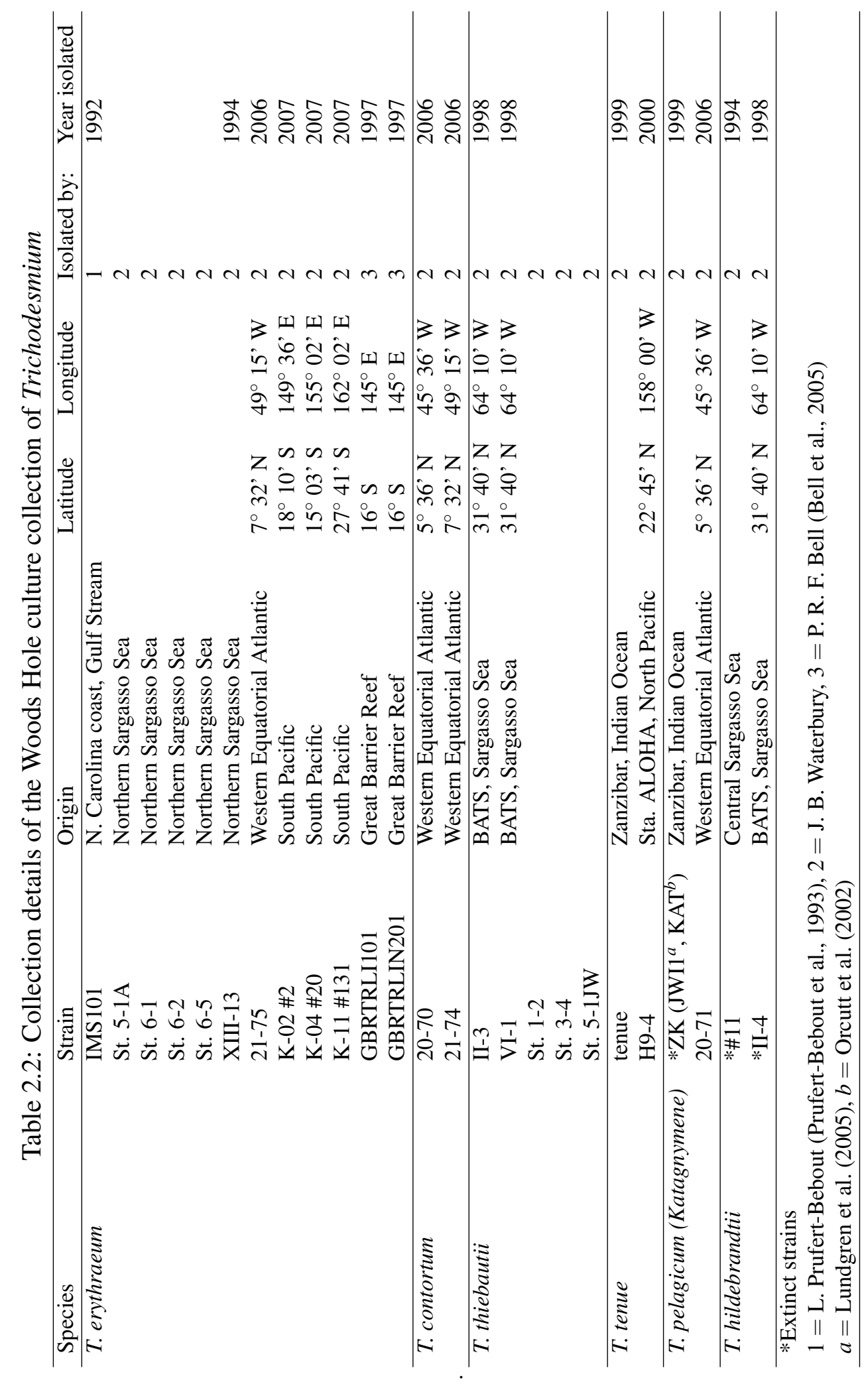




\section{Methods}

Culture conditions. Batch cultures were grown at $26^{\circ} \mathrm{C}, 50 \mu \mathrm{E} \mathrm{m}^{-2} \mathrm{~s}^{-1}$, under soft white fluorescent bulbs with a 14:10 light-dark cycle in RMP medium made from $75 \%$ sterile Sargasso Sea water amended with trace metals and vitamin $\mathrm{B}_{12}$, as described in Webb et al. (2001), and with $2 \times 10^{-6} \mathrm{M}$ EDTA, $15 \times 10^{-6} \mathrm{M}$ phosphoric acid, $1 \times 10^{-7}$ M Fe (ferric citrate). Cultures were maintained in Citranox- and acid-washed $250 \mathrm{~mL}$ polycarbonate baffled flasks closed with cotton stoppers or $75 \mathrm{~mL}$ square polycarbonate culture bottles (Nalgene).

Micrographs. Wet mounts of culture were examined using a Zeiss Axioplan 2 microscope using phase contrast with a Plan-Neofluar $100 \times$ objective lens and differential intereference contrast (DIC) using an $\alpha$ Plan-Fluar $100 \times$ objective lens. Micrographs were taken using a Zeiss Axiocam HRC digital camera and Axiovision 4.6.3 software. Images were measured, and cell metrics were determined using the MATLAB image processing toolbox.

Phycobiliprotein extraction and absorption spectra. Trichodesmium phycobiliprotein extracts were prepared as described in Rippka et al. (1974) with minor modifications. Cultures were filtered on $25 \mathrm{~mm}, 5 \mu \mathrm{m}$ polycarbonate filters and stored at $-20^{\circ} \mathrm{C}$. Two

$\mathrm{mL}$ of $\mathrm{pH} 7.0,5 \mathrm{mmol} \mathrm{L}^{-1}$ phosphate buffer containing $0.1 \mathrm{~mol} \mathrm{~L}^{-1} \mathrm{NaCl}$ were added to each sample and vortexed to remove cells from the filter. Cells were lysed at $4^{\circ} \mathrm{C}$ using a digital sonifier (Branson; $3 \times$ for $1 \mathrm{~min}$ at $65 \%$ with on/off cycles for $0.5 \mathrm{~s}$ each), a tissue homogenizer, or a small French pressure cell (Aminco). The extracts were centrifuged in a Beckman ultracentrifuge for 45 minutes at $112,000 \mathrm{rcf}$ and $4{ }^{\circ} \mathrm{C}$. The supernatant was pipetted into a $1.5 \mathrm{~mL}$ microcentrifuge tube and centrifuged in an Eppendorf 5810R centrifuge for 15 minutes at $20,000 \mathrm{rcf}$ and $4^{\circ} \mathrm{C}$. Seventy-five $\mu \mathrm{L}$ of supernatant was pipetted into a quartz microcuvette and the absorption spectra from 400 - $700 \mathrm{~nm}$ (0.5 nm step) was measured using a Shimadzu UV-1601 spectrophotometer. Blank spectra of the phos- 
phate buffer alone were subtracted from the absorption spectra of the samples. Replicate spectra were averaged and normalized to the maximum absorption.

Sequences and phylogeny. Cultured strains of Trichodesmium were filtered on $25 \mathrm{~mm}$, $5 \mu \mathrm{m}$ polycarbonate filters and stored in liquid $\mathrm{N}_{2}$ until extraction. DNA was extracted using the DNeasy Tissue Kit (Qiagen), following the manufacturer's instructions for animal tissue. Extracted DNA was stored at $-20^{\circ} \mathrm{C}$. All polymerase chain reactions (PCR) were performed on a Biorad iCycler with $25 \mu \mathrm{L}$ reactions. Primers are summarized in Table 2.3. Amplification of $16 \mathrm{~S}$ rDNA was performed using the high-fidelity iProof kit (Biorad) to get a $662 \mathrm{bp}$ fragment. Each reaction contained $1 \times$ HF Buffer, $200 \mu \mathrm{M}$ dNTPs, $0.5 \mu \mathrm{M}$ each of primers CYA-106F and CYA-781R(a) (Nübel et al., 1997), 0.02 U iProof DNA polymerase, and 4 ng of genomic DNA. Reactions were cycled using the temperature profile of $98^{\circ} \mathrm{C}$ for $30 \mathrm{~s} ; 30$ cycles of $98^{\circ} \mathrm{C}$ for $10 \mathrm{~s}, 65.5^{\circ} \mathrm{C}$ for $20 \mathrm{~s}$, and $72^{\circ} \mathrm{C}$ for $20 \mathrm{~s}$; and one cycle at $72^{\circ} \mathrm{C}$ for $7 \mathrm{~min}$. The ITS region was amplified using the MasterTaq Kit (Eppendorf, 5 Prime) to get a $1026 \mathrm{bp}$ fragment. Reactions consisted of $1 \times$ TaqMaster PCR Enhancer; $1 \times$ Taq Buffer; $3.5 \mathrm{mM} \mathrm{Mg}^{2+}$ (including $\mathrm{Mg}^{2+}$ in the Taq Buffer); $200 \mu \mathrm{M}$ dNTPs; $0.5 \mu \mathrm{M}$ each of primer tri16S-1247F (Orcutt et al., 2002) and primer tri-23SR, modified from 23S-241R (Rocap et al., 2002) to be specific to Trichodesmium based on the T. erythraeum IMS101 genome; $1.25 \mathrm{U}$ Taq DNA polymerase; and 4 ng genomic DNA. Thermal cycling conditions for ITS were $95^{\circ}$ for $2 \mathrm{~min}$; 30 cycles of $95^{\circ}$ for $1 \mathrm{~min}$, $55^{\circ}$ for $1 \mathrm{~min}$, and $72^{\circ}$ for $1 \mathrm{~min}$; and a final cycle of $72^{\circ}$ for $10 \mathrm{~min}$. Amplification of $h e t R$ was conducted using the iProof kit (Biorad) to get a $448 \mathrm{bp}$ fragment. The reaction mixture was made up of $1 \times$ HF Buffer, $200 \mu \mathrm{mol} \mathrm{L}^{-1} \mathrm{dNTP}, 0.5 \mu \mathrm{mol} \mathrm{L} \mathrm{L}^{-1}$ each of primers PH1 and PH2 (Lundgren et al., 2005), and $0.02 \mathrm{U}_{\mu \mathrm{L}^{-1}}$ iProof DNA polymerase, and 4 ng of genomic DNA. Cycling conditions for hetR were $98^{\circ}$ for $30 \mathrm{~s} ; 30$ cycles of $98^{\circ}$ for $10 \mathrm{~s}, 57.7^{\circ}$ for $20 \mathrm{~s}$, and $72^{\circ}$ for $20 \mathrm{~s}$; and one cycle of $72^{\circ}$ for $7 \mathrm{~min}$. 
Table 2.3: Summary of PCR primers.

\begin{tabular}{lllll}
\hline Gene & \multicolumn{2}{l}{ Primers } & Sequence (5' to 3') & Source \\
\hline $16 \mathrm{~S}$ & For & CYA-106F & CGGACGGGTGAGTAACGCGTGA & (Nübel et al., 1997) \\
& Rev & CYA-781R(a) & GACTACTGGGGTATCTAATCCCATT & (Nübel et al., 1997) \\
ITS (external) & For & tri16S-1247F & CGTACTACAATGGTTGGG & (Orcutt et al., 2002) \\
& Rev & tri-23SR & TTCGCTCACCGCTACA & This study \\
\multirow{2}{*}{ ITS (internal) } & For & AlaF & TWTAGCTCAGTTGGTAGAG & (Rocap et al., 2002) \\
& Rev & AlaR & CTCTACCAACTGAGCTAWA & (Rocap et al., 2002) \\
\multirow{2}{*}{ hetR } & For & PH1 & TGYGCKATTTAYATGACCTA & (Lundgren et al., 2005) \\
& Rev & PH2 & ATGAANGGTATKCCCCAA GGA & (Lundgren et al., 2005) \\
\hline
\end{tabular}

Five $\mu \mathrm{L}$ of each PCR product was separated on a $1 \%$ agarose gel, stained with ethidium bromide, and photographed using a Gel Logic 200 Imaging System and Molecular Imaging Software (Kodak). 16S, hetR, and ITS samples with only one band were purified using a QIAquick PCR Purification Kit (Qiagen). Samples of ITS that showed multiple bands were separated on 1\% agarose gels stained with ethidium bromide and purified using the QIAquick Gel Extraction Kit (Qiagen). Sequencing of purified PCR products in both directions was performed in duplicate or triplicate for each primer on a 3730XL capillary sequencer (Applied Biosystems) by the Keck Facility at the Josephine Bay Paul Center, Marine Biological Laboratory, Woods Hole. BigDye Terminator v3.1 chemistry was used in $\frac{1}{16}^{\text {th }}$ reactions: $6 \mu \mathrm{L}$ total volume with $1 \times$ Sequencing Buffer, 5 pmol primer (Table 2.3), 0.5 $\mu \mathrm{L}$ BigDyeTerminator v3.1, $0.1 \mu \mathrm{L}$ DMSO, and 10 - $30 \mathrm{ng}$ purified PCR product. Thermal cycling conditions were 60 cycles at $96^{\circ} \mathrm{C}$ for $10 \mathrm{~s}, 50^{\circ} \mathrm{C}$ for $5 \mathrm{~s}$, and $60^{\circ}$ for $4 \mathrm{~min}$. Sequencing reactions were precipitated with isopropanol and resuspended in $5 \mu \mathrm{L}$ Hi-Di formamide (Applied Biosystems). To get complete coverage of the ITS region, internal primers AlaF and AlaR, were also used (Rocap et al., 2002). A few of the 16S and hetR purified PCR products were sent to Northwoods DNA, Inc. for sequencing.

Sequences were edited using Sequencher 4.6 and aligned using either ClustalX or the MATLAB Bioinformatics Toolbox. Alignments were edited in either MacVector 8.0 or the MATLAB multiple alignment viewer and similarity matrices were calculated, rounding to the nearest integer. Sequences acquired from GenBank are listed be- 
low with accession numbers in parenthesis (Benson et al., 2005). The $16 \mathrm{~S}$ alignment was trimmed to $564 \mathrm{bp}$ and included field samples T. hildebrandtii (AF091322), T. contortum (AF013028), and T. tenue (AF013029) from Janson et al. (1999); the Woods Hole cultured strain T. pelagicum ZK (AF518769); field sample Trichodesmium havanum (AF518770) from Lundgren et al. (2005); and cultured strain Oscillatoria sancta PCC 7515 (AY768400). The ITS alignment was trimmed to exclude the 16S and 23S sequences (497 bp). Trichodesmium ITS includes the alanine and isoleucine tRNA gene sequences, which were identical among the strains and included in the phylogeny. The alignment included Woods Hole cultured strains T. pelagicum ZK (AF399652) and T. hildebrandtii II4 (AF399650) from Orcutt et al. (2002), and cultured strain O. sancta PCC 7515 (EF178272). The hetR alignment was trimmed to $447 \mathrm{bp}$ and included field samples T. pelagicum (AF490696), T. hildebrandtii (AF490679), Trichodesmium aureum (AF490680), and T. aureum (AF490682) from Lundgren et al. (2005); field samples T. contortum (AF013031), T. contortum (AF490685), and T. tenue (AF013033) from Janson et al. (1999); and Arthrospira platensis HZ01 (EU427544). 16S, ITS, and hetR sequences were concatenated to form a 1507 bp sequence (Gadagkar et al., 2005). The concatenated alignment included GenBank sequences for field samples of T. hildebrandtii (AF490679, AF399650, and AF490679) and the $16 \mathrm{~S}$ sequence for cultured strain T. pelagicum ZK (AF518769). Phylogenetic trees were created for each genetic marker and the concatenated alignment in Paup 4.0b10 using parsimony, neighbor-joining, and maximum likelihood methods and rooted using the sequences from the closely-related $O$. sancta or A. platensis (order Oscillatoriales). Each tree was bootstrapped 2000 times except for the ITS maximum likelihood tree, which proved to be computationally intensive. Trees were edited using Mesquite 2.6 (http://mesquiteproject.org).

\section{Results}

Genetic characterization of Trichodesmium. Two distinct clades of Trichodesmium could be distinguished using each of the three genetic markers: clade I containing T. ery- 
thraeum and T. contortum, and clade II containing T. thiebautii, T. tenue, T. pelagicum, and T. hildebrandtii (Figs. 2-3-2-14).

The partial 16S sequences were highly similar for all strains of Trichodesmium ( $\geq 97 \%$ ). Similarity to the outgroup $O$. sancta ranged from $95-97 \%$. For clade I, intraclade similarities were between 99 and $100 \%$. Subgroups of clade I found consistently in all three phylogenetic analyses were (1) T. contortum strains 20-70 and 21-74 and $T$. havanum; and (2) T. erythraeum strains XIII-13, St. 5-1A, St. 6-1, St. 6-2, and St. 6-5 (Figs. 2-3-2-5). The $16 \mathrm{~S}$ sequence for T. pelagicum 20-71 was not successful. Within clade II, sequence similarities were $100 \%$ with the exception of T. hildebrandtii, which was $99 \%$ similar to all the other sequences in the clade. Trichodesmium sp. H9-4, T. tепие tenue, and T. pelagicum ZK formed a consistent subgroup of clade II and had one basepair difference from the other sequences in the clade (Figs. 2-3-2-5). Field samples of $T$. contortum and T. tenue from Janson et al. (1999) formed their own clade (Figs. 2-3 - 2-5).

The ITS region is $\geq 89 \%$ identical among all strains of Trichodesmium and similarity to the outgroup $O$. sancta was $80-81 \%$. Similarities between the two major clades of Trichodesmium were $89-90 \%$. The three phylogenetic analyses showed slightly different topologies. The clade I sequences were $\geq 98 \%$ identical. The neighbor-joining and parsimony analyses had a subgroup of clade I containing T. erythraeum strains St. 5-1A, St. 6-1, St. 6-2, and St. 6-5 (Figs. 2-6, 2-7), but the maximum likelihood analysis did not (Fig. 2-8). The maximum likelihood analysis yielded a clade I subgroup containing $T$. erythraeum strains IMS101, 21-75, K-02 \#2, GBRTRLI101, and GBRTRLIN201 (Fig. $2-8)$. Sequences within clade II were 98 - 100\% identical. All three trees showed a subgroup of clade II containing T. thiebautii strains II-3, VI-1 and St. 3-4 (Figs. 2-6 - 2-8). Only the neighbor-joining analysis contained a clade II subgroup of Trichodesmium sp. H9-4, T. tenue tenue, and T. pelagicum ZK (Fig. 2-6).

Trichodesmium hetR sequences were $\geq 90 \%$ identical and were $74-75 \%$ similar to the outgroup Arthrospira platensis. Inter-clade similarity between clades I and II was $90-91 \%$. Members of clade I were 100\% identical except for T. erythraeum 21-75, 
which was $99 \%$ similar to the other strains. All three phylogenetic analyses clustered $T$. erythraeum strains K-11 \#131, St. 5-1A, St. 6-1, St. 6-2, and St. 6-5 together as a subgroup of clade I (Figs. 2-9-2-11). Intra-clade similarity in clade II was 97 - 100\%. All three trees had subgroups of clade II consisting of (1) T. thiebautii strains St. 3-4 and VI1, and (2) T. thiebautii strains St. 1-2 and St. 5-1JW and T. hildebrandtii (Figs. 2-9-2-11). The neighbor-joining tree also showed a clade II subgroup containing T. thiebautii II-3, T. tenue tenue, and Trichodesmium H9-4 (Fig. 2-9). The Janson et al. (1999) field samples of $T$. contortum and T. tenue formed their own clade as did the Lundgren et al. (2005) field samples of T. aureum.

The concatenated Trichodesmium 16S-ITS-hetR sequences were $\geq 93 \%$ identical. Inter-clade similarities were $93 \%$ and intra-clade similarities were 99 - 100\%. In clade I, all three phylogenetic analyses showed subgroups of (1) T. erythraeum strains St. 5-1A, St. 6-1, St. 6-2 and St. 6-5; and (2) T. contortum strains 20-70 and 21-74 (Figs. 2-122-14). Within clade II, the subgroups (1) T. tenue tenue and Trichodesmium sp. H9-4, and (2) Trichodesmium strains St. 1-2 and St. 5-1JW and T. hildebrandtii were consistent across all three trees (Figs. 2-12-2-14). The neighbor-joining and parsimony analyses also yielded a clade II subgroup containing T. thiebautii strains VI-1 and St. 3-4 (Figs. 2-12-2-13). 


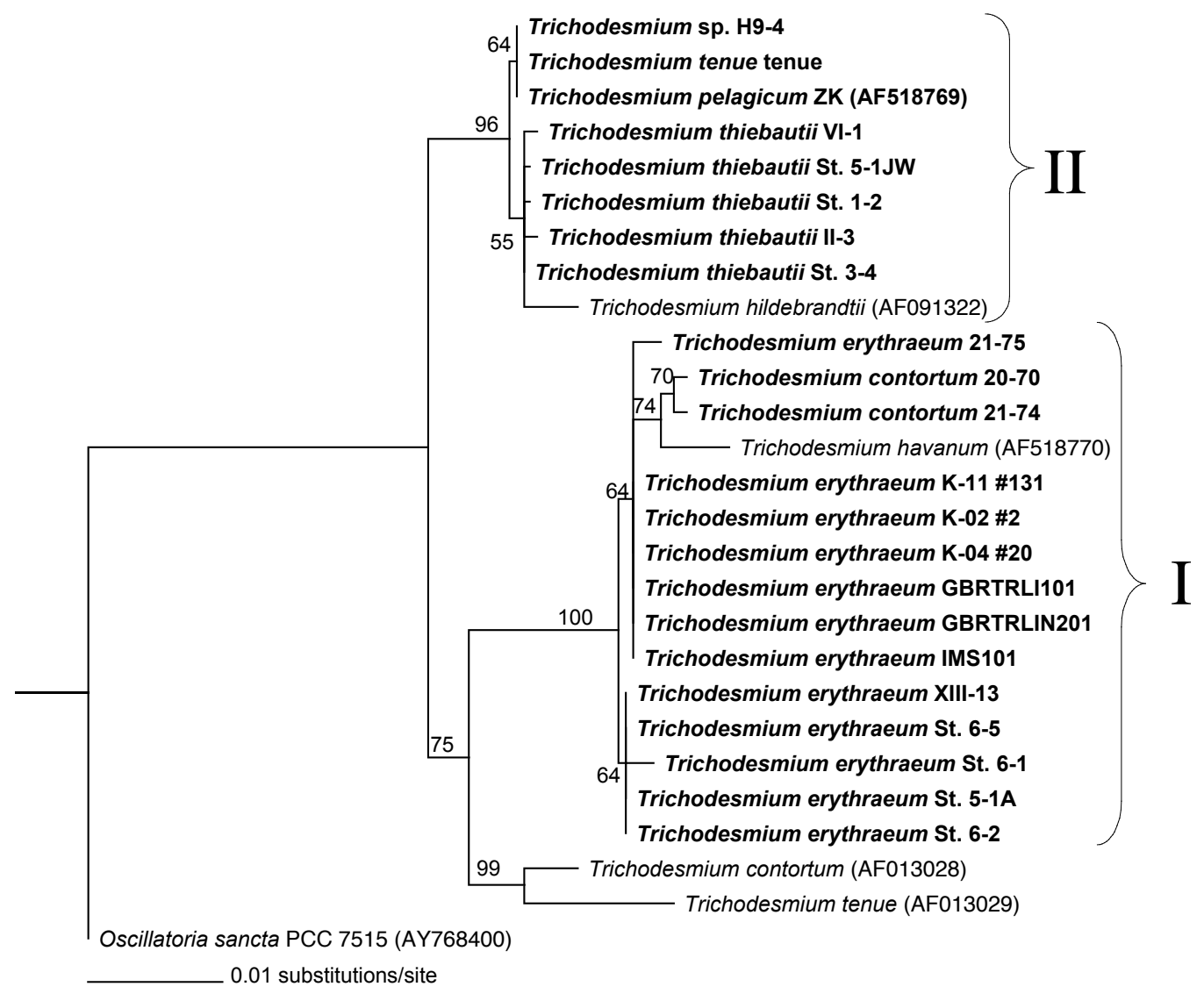

Figure 2-3: $16 \mathrm{~S}$ rDNA tree analyzed by neighbor-joining. The tree was bootstrapped 2000 times and Oscillatoria sancta PCC 7515 was used as an outgroup to root the tree. Numbers at nodes reflect percent bootstrap consensus $>50 \%$. Boldfaced text is used to indicate sequences from the Woods Hole culture collection. Sequences from GenBank are indicated by accession numbers in parentheses. 


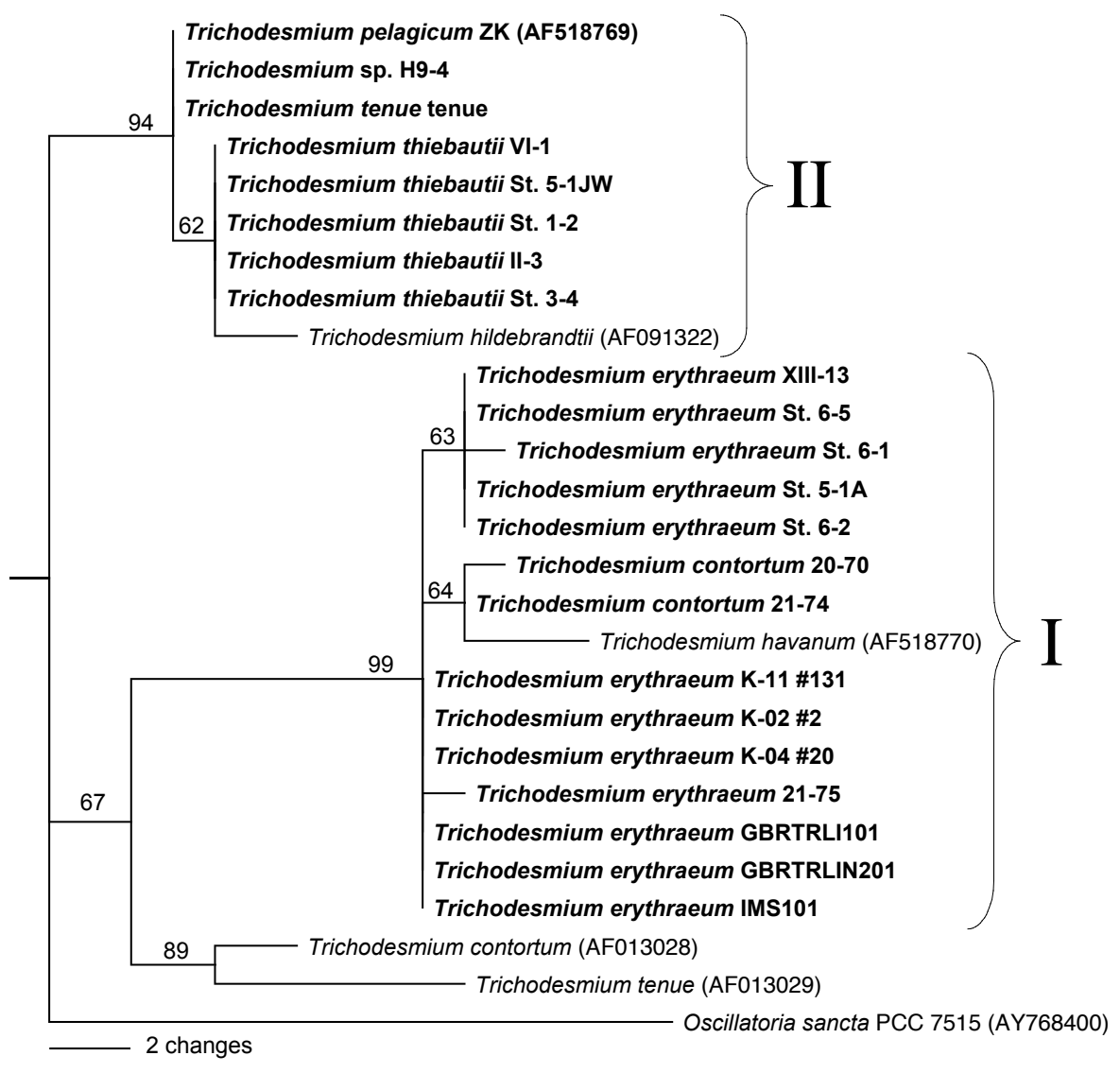

Figure 2-4: 16S rDNA tree based on parsimony. The tree was bootstrapped 2000 times and Oscillatoria sancta PCC 7515 was used as an outgroup to root the tree. Numbers at nodes reflect percent bootstrap consensus $>50 \%$. Boldfaced text is used to indicate sequences from the Woods Hole culture collection. Sequences from GenBank are indicated by accession numbers in parentheses. 


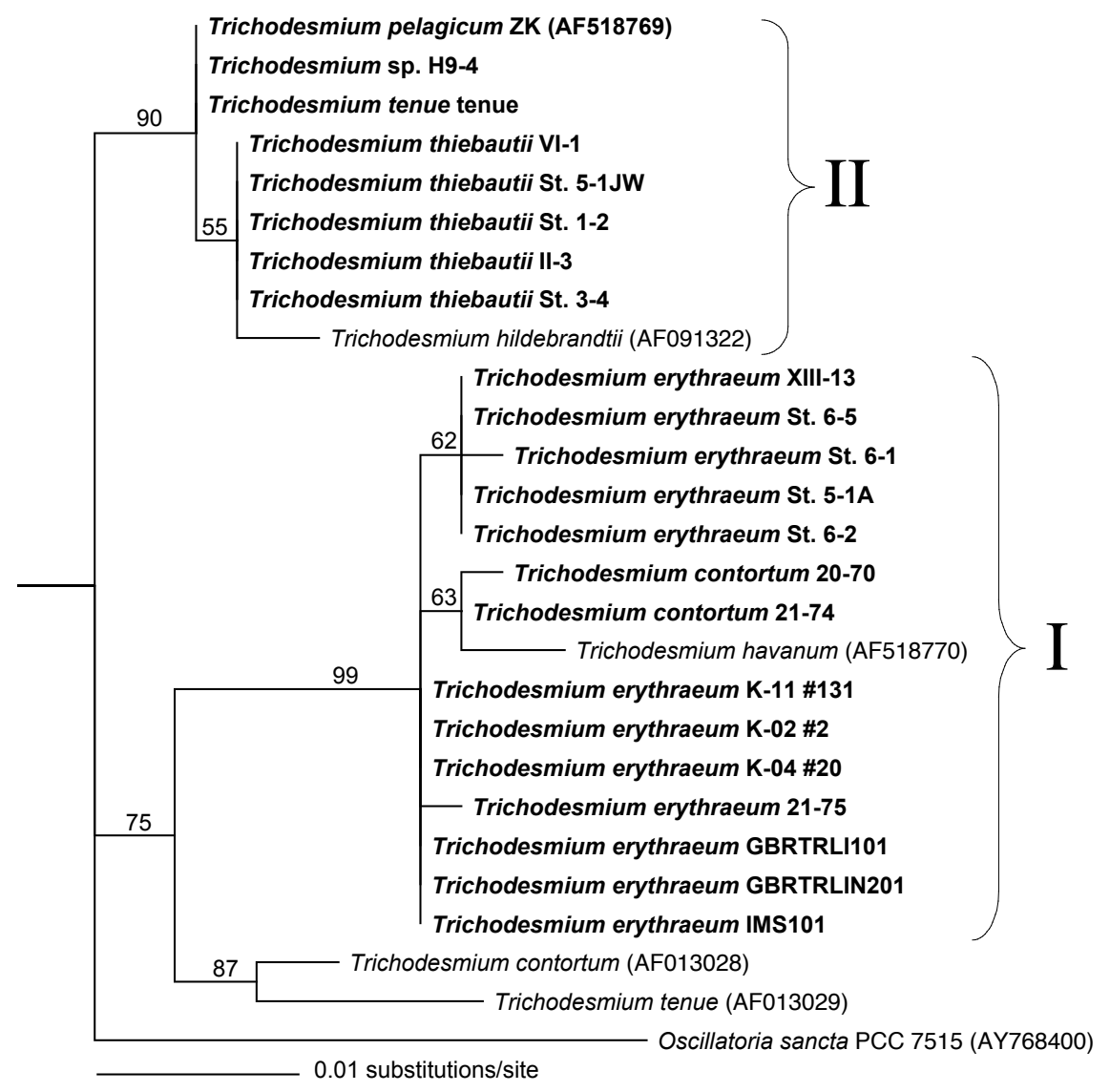

Figure 2-5: 16S rDNA tree based on maximum likelihood. The tree was bootstrapped 2000 times and Oscillatoria sancta PCC 7515 was used as an outgroup to root the tree. Numbers at nodes reflect percent bootstrap consensus $>50 \%$. Boldfaced text is used to indicate sequences from the Woods Hole culture collection. Sequences from GenBank are indicated by accession numbers in parentheses. 


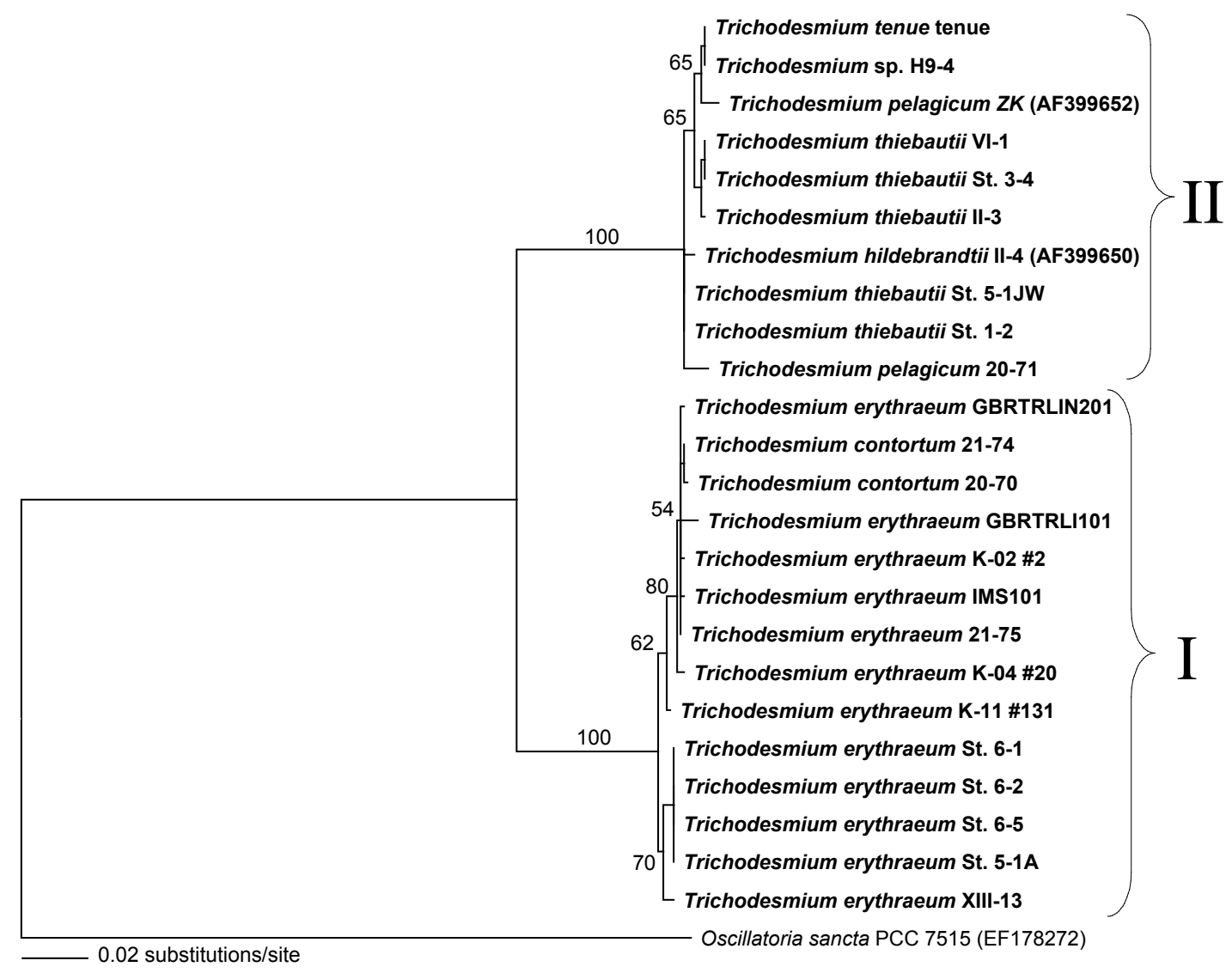

Figure 2-6: ITS tree analyzed by neighbor-joining. The tree was bootstrapped 2000x and Oscillatoria sancta PCC 7515 was used as an outgroup to root the tree. Bootstrap values $>50 \%$ are indicated at the nodes as space allowed. The bootstrap value for the T. tenue tenue and Trichodesmium sp. H9-4 node is 91; the value for the T. thiebautii VI-1, T. thiebautii St. 3-4, and T. thiebautii II-3 node is 64; the value for the T. thiebautii VI-1, T. thiebautii St. 3-4 node is 67; the value for the T. contortum 21-74 and T. contortum 20-70 node is 77; and the value for the T. erythraeum St. 6-1, T. erythraeum St. 6-2, T. erythraeum St. 6-5, and T. erythraeum St. 5-1A is 99. Boldfaced text is used to indicate sequences from the Woods Hole culture collection. Sequences from GenBank are indicated by accession numbers in parentheses. 


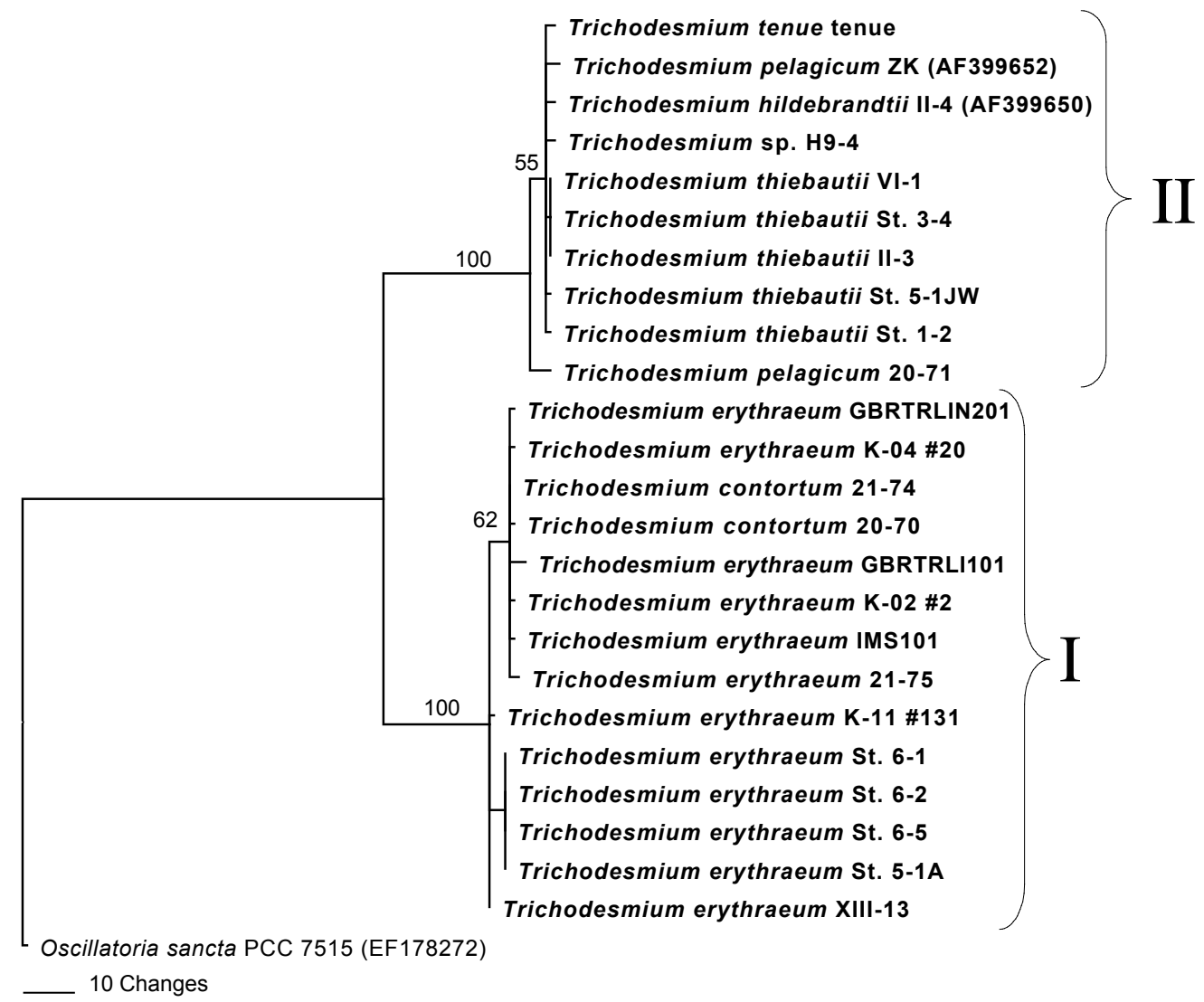

Figure 2-7: ITS tree analyzed by parsimony tree. The tree was bootstrapped 2000 times and rooted using Oscillatoria sancta PCC 7515. Bootstrap values $>50 \%$ are indicated at the nodes as space allowed. The bootstrap value for the T. thiebautii VI-1, T. thiebautii St. 3-4, and T. thiebautii II-3 node is 62 and the value for the T. erythraeum St. 6-1, T. erythraeum St. 6-2, T. erythraeum St. 6-5, and T. erythraeum St. 5-1A is 73. Boldfaced text is used to indicate sequences from the Woods Hole culture collection. Sequences from GenBank are indicated by accession numbers in parentheses. 


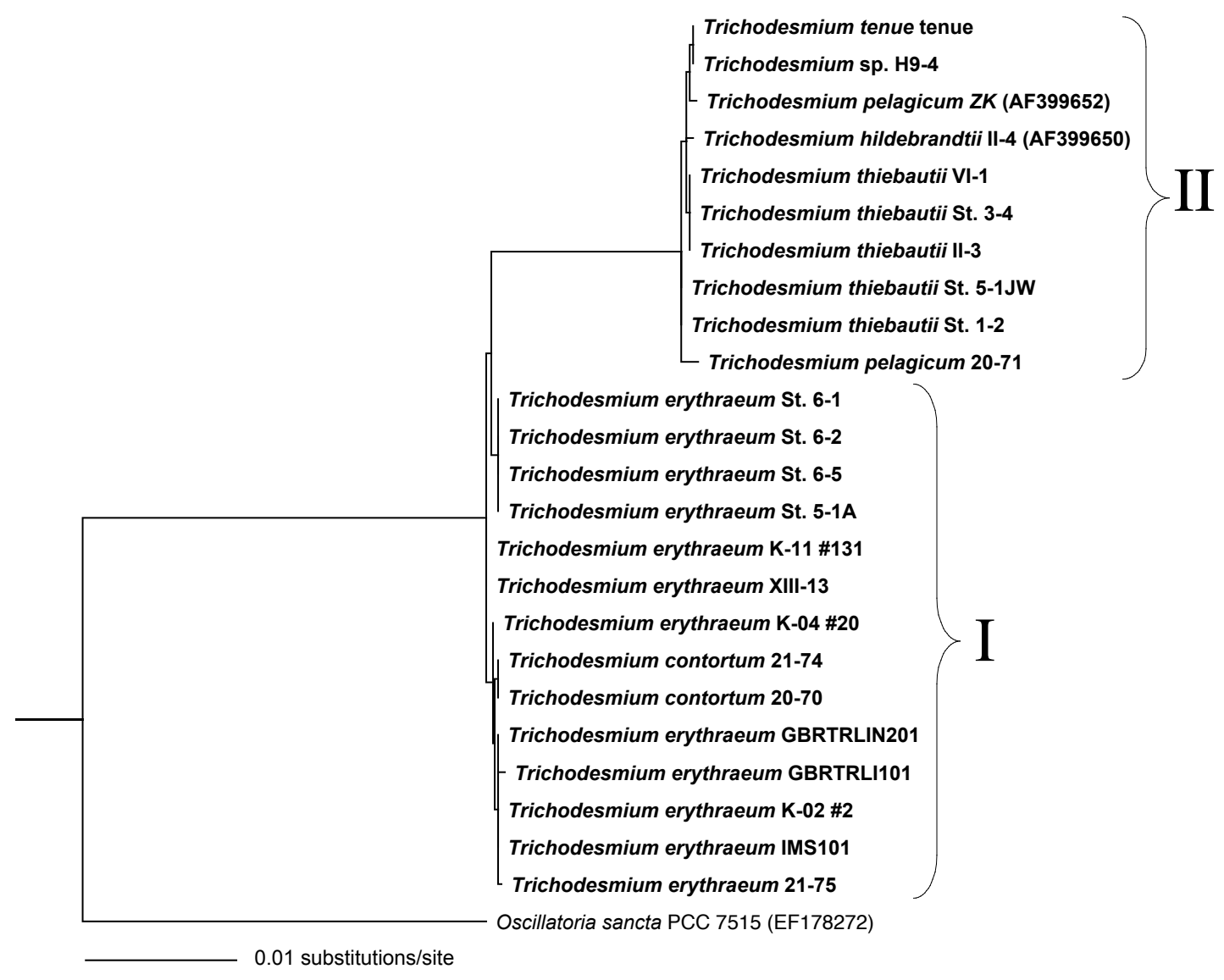

Figure 2-8: ITS tree analyzed by maximum likelihood. Oscillatoria sancta PCC 7515 was used as an outgroup to root the tree. Boldfaced text is used to indicate sequences from the Woods Hole culture collection. Sequences from GenBank are indicated by accession numbers in parentheses. This tree was not bootstrapped. 


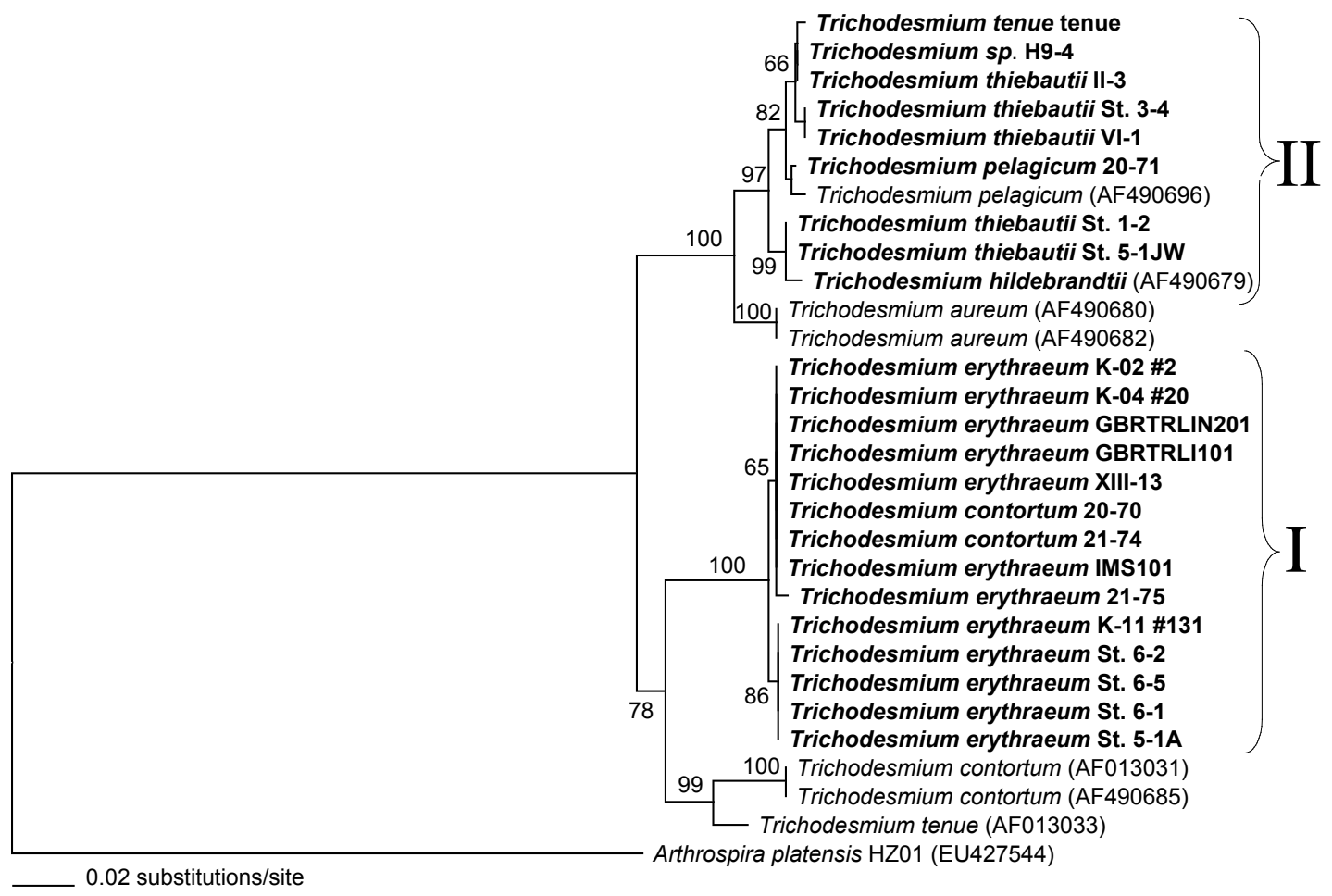

Figure 2-9: hetR tree based on neighbor-joining. The tree was bootstrapped 2000 times and rooted using Arthrospira platensis HZ01. Bootstrap values $>50 \%$ are indicated at the nodes as space allowed. The bootstrap value for the Trichodesmium tenue tenue, Trichodesmium sp. H9-4, and T. thiebautii II-3 node is 85; the value for the Trichodesmium sp. H9-4 and T. thiebautii II-3 node is 68; and the value for the T. thiebautii St. 3-4 and VI-1 node is 88 . Boldfaced text is used to indicate sequences from the Woods Hole culture collection. Sequences from GenBank are indicated by accession numbers in parentheses. 


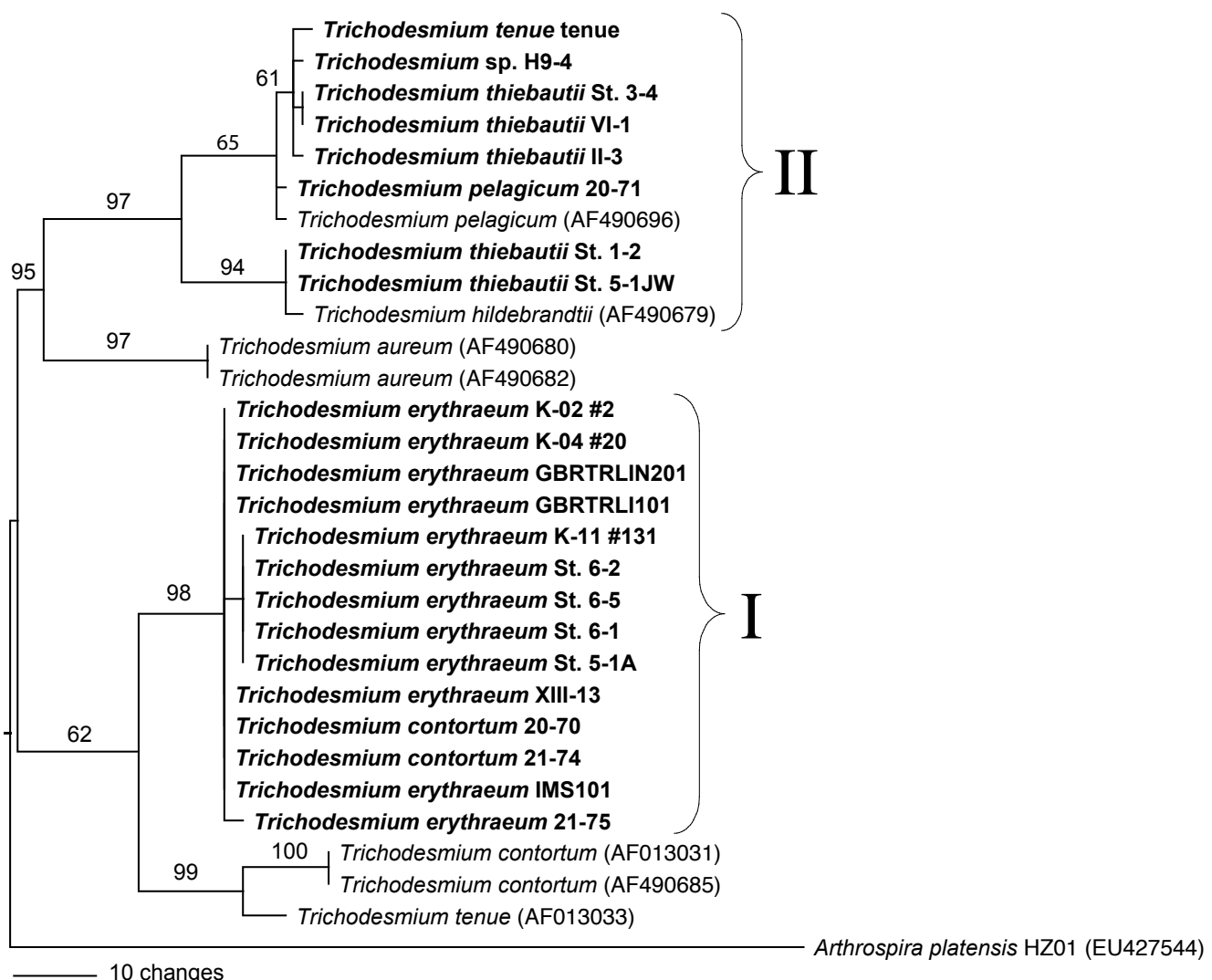

Figure 2-10: hetR tree based on parsimony. The tree was bootstrapped 2000 times and rooted using Arthrospira platensis HZ01. Bootstrap values $>50 \%$ are indicated at the nodes as space allowed. The bootstrap value for the T. thiebautii St. 3-4 and VI-1 node is 63 and the value for the T. erythraeum K-11 \#131, St. 6-2, St. 6-5, St. 6-1, and St. 5-1A node is 78. Boldfaced text is used to indicate sequences from the Woods Hole culture collection. Sequences from GenBank are indicated by accession numbers in parentheses. 


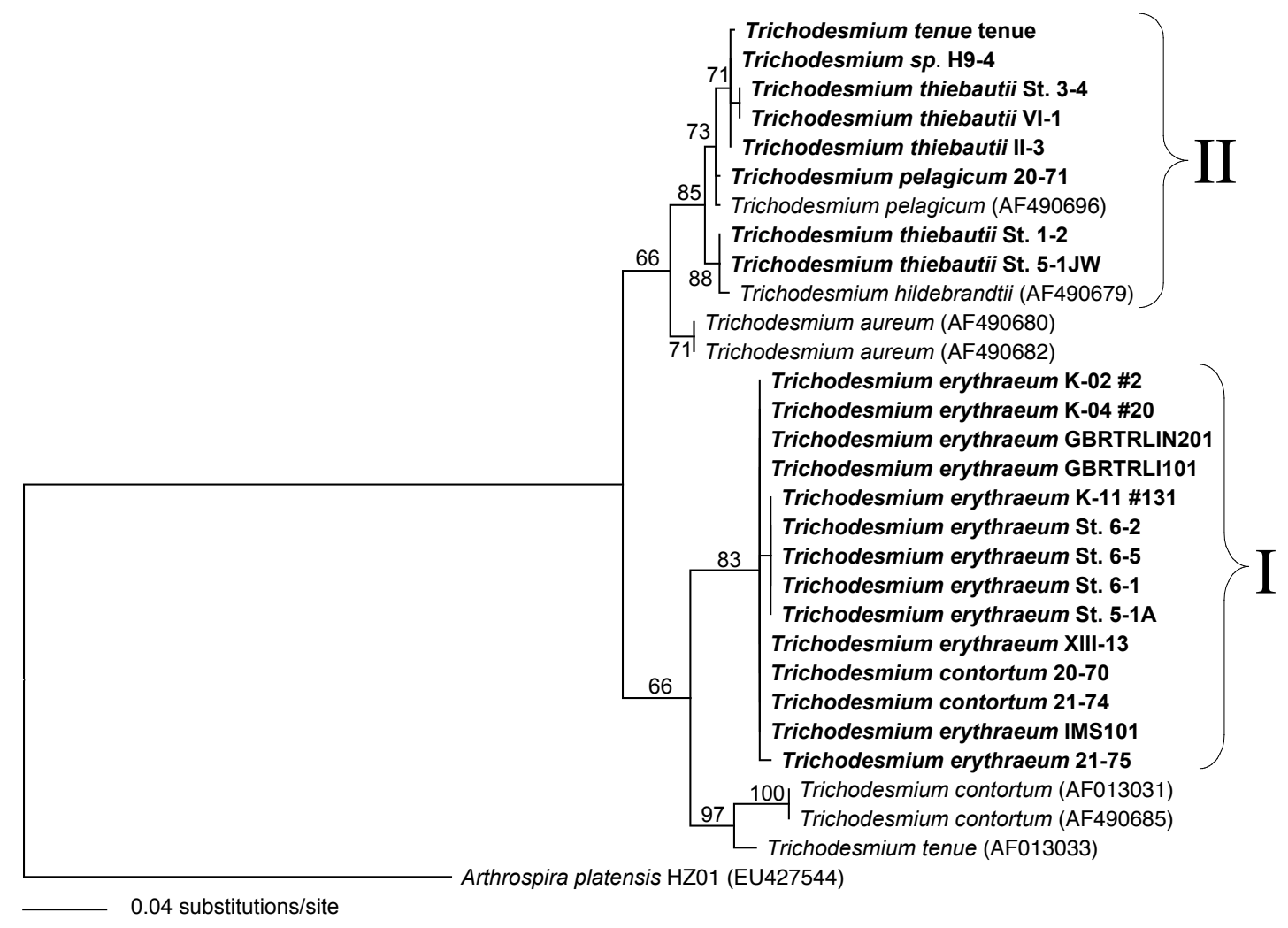

Figure 2-11: hetR tree based on maximum likelihood. The tree was bootstrapped 2000 times and rooted using Arthrospira platensis HZ01. Bootstrap values $>50 \%$ are indicated at the nodes as space allowed. The bootstrap value for the T. thiebautii St. 3-4 and VI-1 node is 63 and the value for the T. erythraeum K-11 \#131, St. 6-2, St. 6-5, St. 6-1, and St. 5-1A node is 63. Boldfaced text is used to indicate sequences from the Woods Hole culture collection. Sequences from GenBank are indicated by accession numbers in parentheses. 


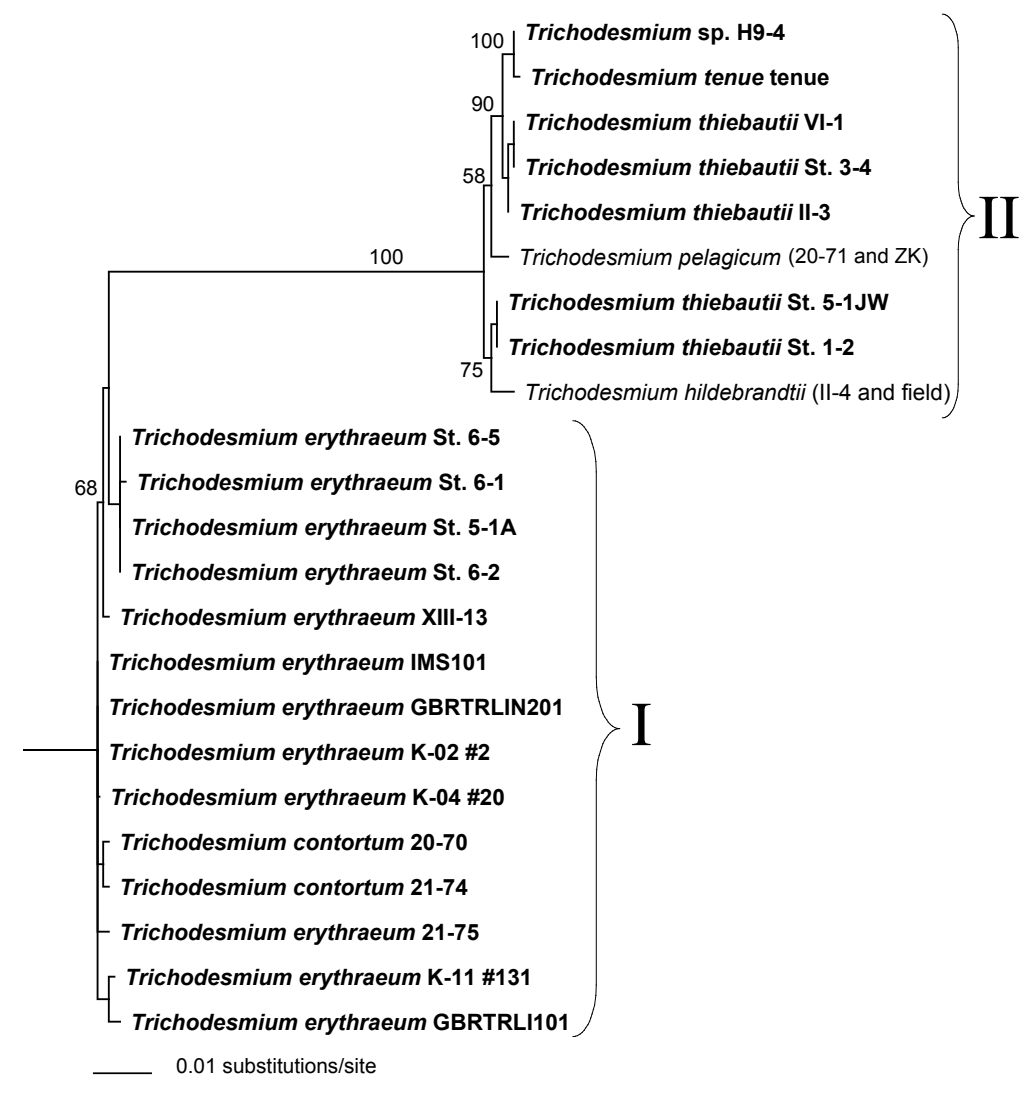

Figure 2-12: Tree of concatenated sequences of $16 \mathrm{~S}$, ITS and het $R$ analyzed by neighborjoining. The tree was bootstrapped 2000 times and percent bootstrap consensus values $>$ $50 \%$ are indicated at the nodes as space allowed. The bootstrap value for the T. thiebautii VI-1, T. thiebautii St. 3-4, and T. thiebautii II-3 node is 65; the value for the T. thiebautii VI-1 and T. thiebautii St. 3-4 node is 96; the value for the T. thiebautii St. 5-1JW and T. thiebautii St. 1-2 is 76; and the value for the T. erythraeum St. 6-5, T. erythraeum St. 6-1, T. erythraeum St. 5-1A, and T. erythraeum St. 6-2 is 96. Sequences which originated completely from this study are in boldface. Sequences which contain sequences from GenBank are indicated by normal text. 


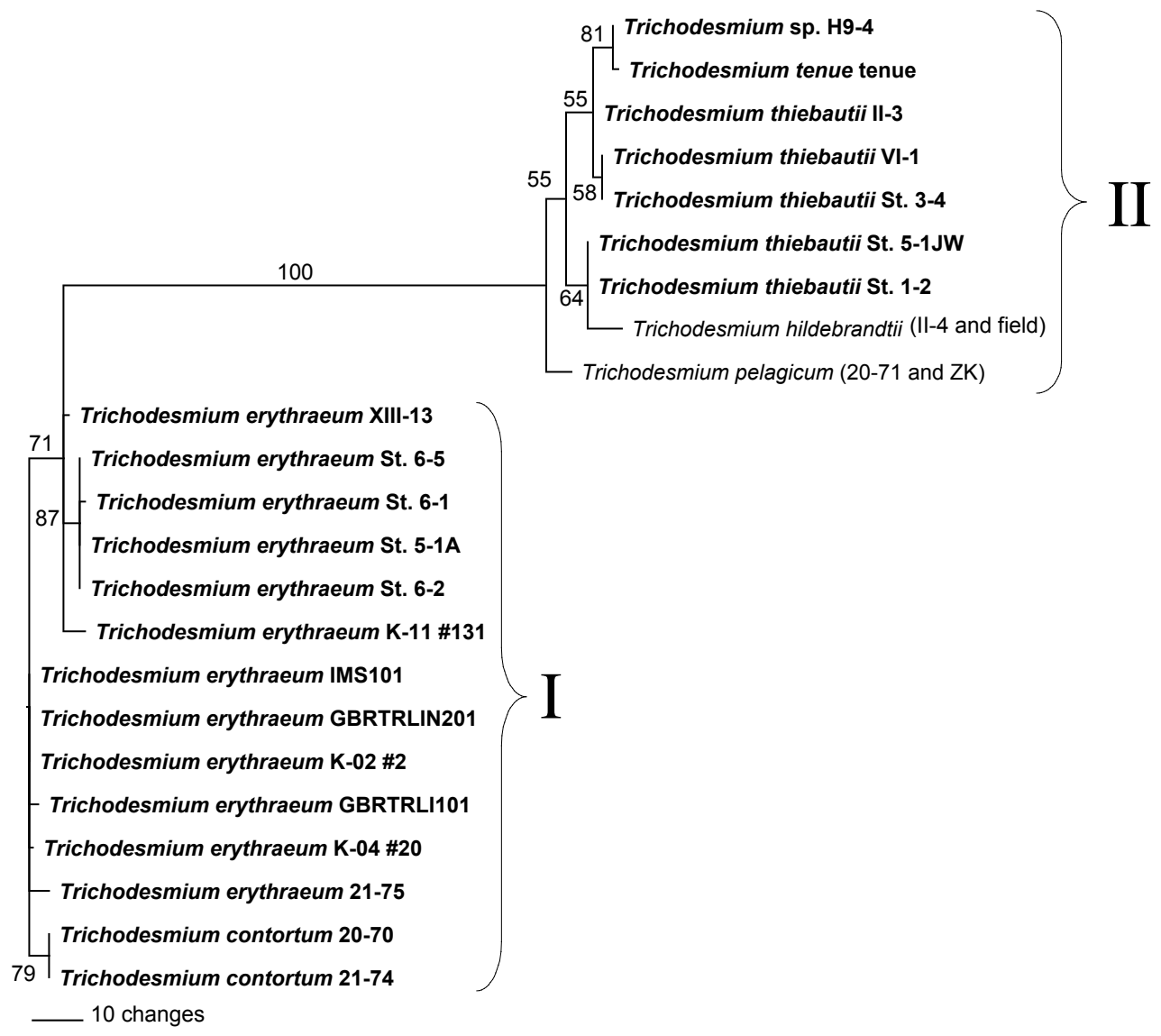

Figure 2-13: Tree of concatenated sequences of $16 \mathrm{~S}$, ITS and het $R$ analyzed by parsimony tree. The tree was bootstrapped 2000 times and percent bootstrap consensus values $>$ $50 \%$ are indicated at the nodes. Sequences which originated completely from this study are in boldface. Sequences which contain sequences from GenBank are indicated by normal text. 


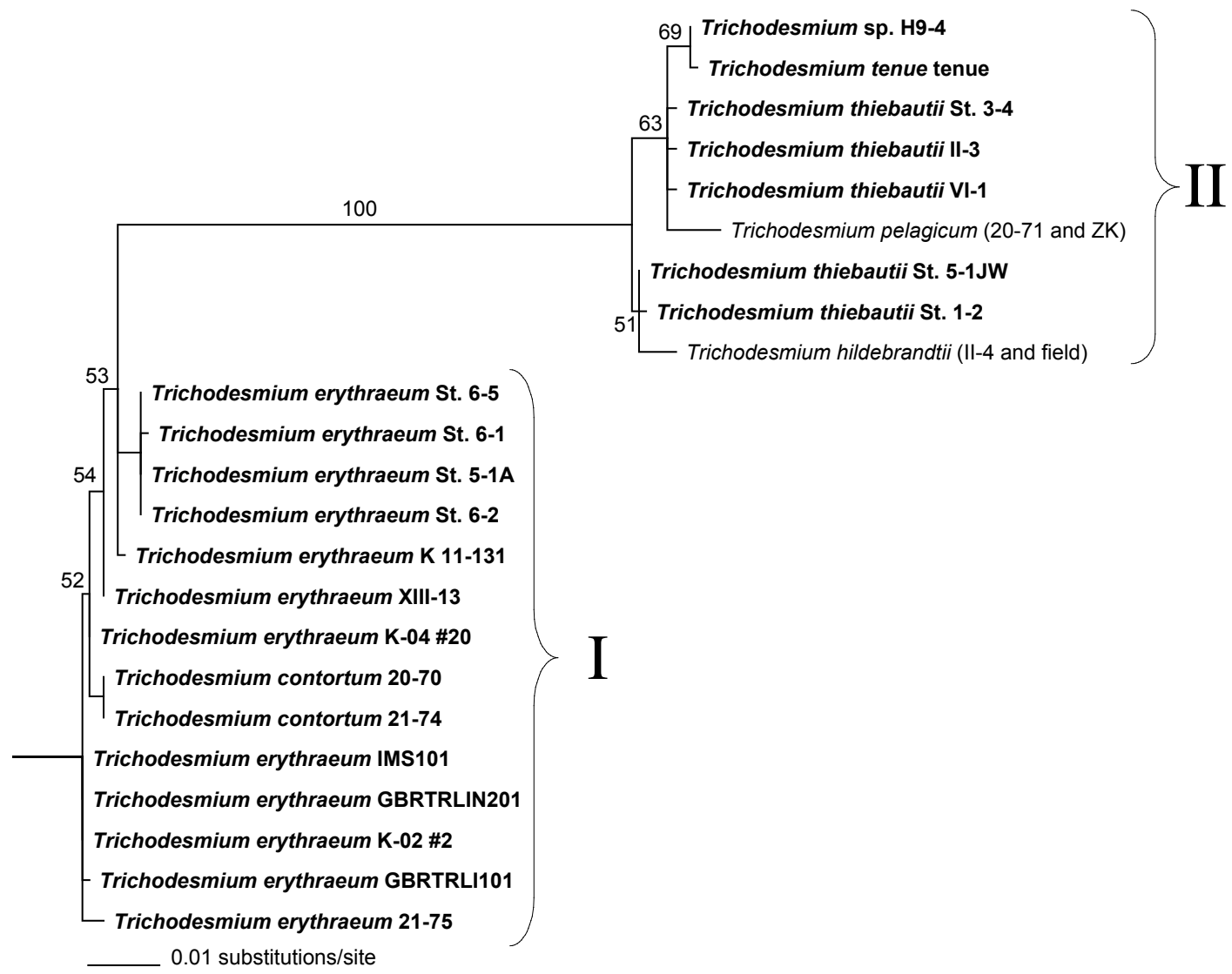

Figure 2-14: Tree of concatenated sequences of 16S, ITS and hetR analyzed by maximum likelihood. The tree was bootstrapped 2000 times and percent bootstrap consensus values $>50 \%$ are indicated at the nodes as space allowed. The bootstrap value for the T. erythraeum St. 6-5, T. erythraeum St. 6-1, T. erythraeum St. 5-1A, and T. erythraeum St. 6-2 node is 70 and the value for the T. contortum 20-70 and T. contortum 21-74 node is 76 . Sequences which originated completely from this study are in boldface. Sequences which contain sequences from GenBank are indicated by normal text. 
Cell morphology. Both clades of Trichodesmium exhibited wide diversity in cell morphology. In clade I, the cell shape of all strains of T. erythraeum ranged from square to wider than long (Table 2.4, Fig. 2-15). Widths ranged from 5.3 - $13.6 \mu \mathrm{m}$ while lengths ranged from 3.9 - $12.2 \mu \mathrm{m}$. Clade I T. contortum cells were all disc-shaped with widths ranging from 19.5 - $34.5 \mu \mathrm{m}$ and lengths ranging from 2.0 - $4.3 \mu \mathrm{m}$ (Table 2.4, Fig. 2-15). Clade II cell shapes ranged from being slightly wider than long to being longer than wide (Table 2.4, Fig. 2-16). Cell widths in T. thiebautii strains ranged from $6.0-11.8 \mu \mathrm{m}$ and lengths ranged from $5.6-22.4 \mu \mathrm{m}$. T. tenue tenue was longer than wide (7.9 - $10.3 \mu \mathrm{m}$ compared to 5.0 - $7.9 \mu \mathrm{m}$, respectively), while Trichodesmium sp. H9-4 was shorter than wide (4.4 - $8.6 \mu \mathrm{m}$ compared to 5.7 - $8.9 \mu \mathrm{m}$, respectively). T. pelagicum 20-71 was the largest of the clade at 12.1 - $18.4 \mu \mathrm{m}$ wide and 5.2 - $9.6 \mu \mathrm{m}$ long (Table 2.4, Fig. 2-16). Gas vesicles can be seen as bright regions under differential interference contrast (DIC) (Figs. 2-15, 2-16). Gas vesicle distribution within the clade I varied from random distribution in strains IMS101, 20-70, and 21-74 to peripheral distribution in all the other strains in the clade. Clade II gas vesicle distribution ranged from random distribution in strains St. 1-2, St. 5-1JW, tenue, and 20-71 to peripheral distribution in strains II-3, VI-1, St. 3-4, and H9-4 (Fig. 2-16). 


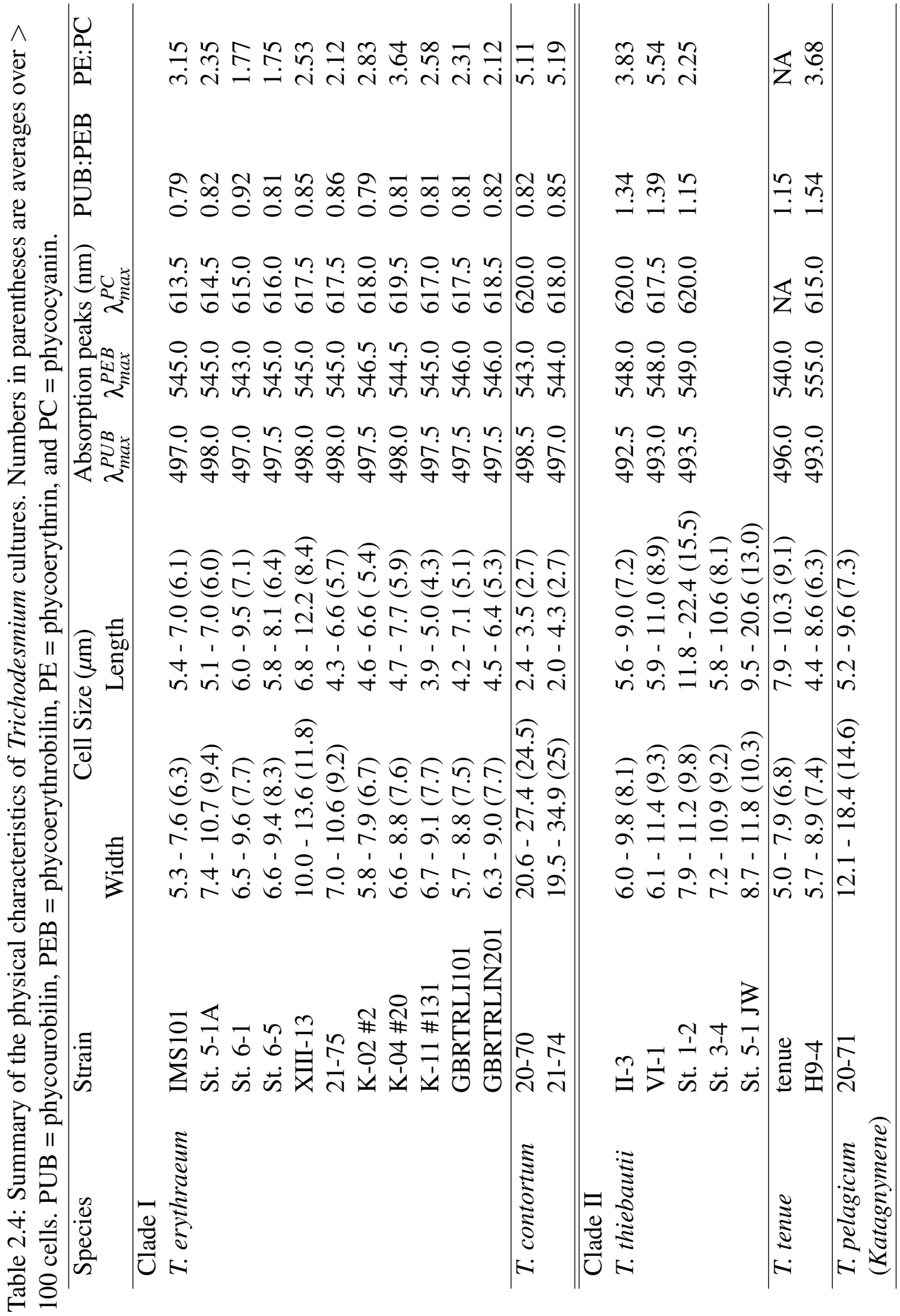




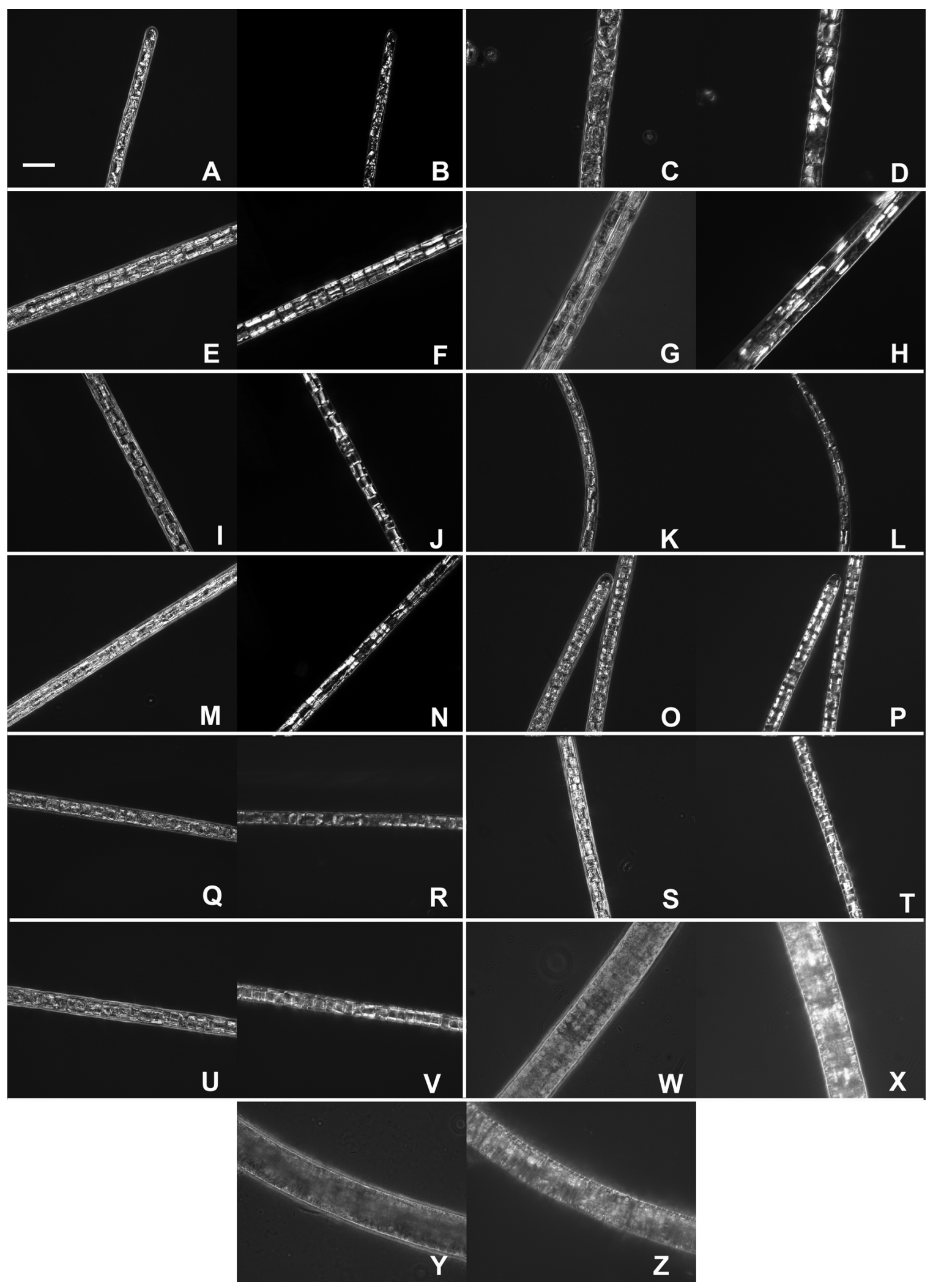


Figure 2-15: Trichodesmium clade I micrographs taken using phase contrast and differential interference contrast (DIC): T. erythraeum (A - V) and T. contortum (W - Z). The white bar in (A) represents $20 \mu \mathrm{m}$. (A) IMS101, phase contrast; (B) IMS101, DIC; (C) XIII-13, phase contrast; (D) XIII-13, DIC; (E) St. 5-1A, phase contrast; (F) St. 5-1A, DIC; (G) St. 6-1, phase contrast; (H) St. 6-1, DIC; (I) St. 6-5, phase contrast; (J) St. 6-5, phase contrast; (K) K-02 \#2, phase contrast; (L) K-02 \#2, DIC; (M) K-04 \#20, phase contrast; (N) K-04 \#20, DIC; (O) K-11 \#131, phase contrast; (P) K-11 \#131, DIC; (Q) GBRTRLI101, phase contrast; (R) GBRTRLI101, DIC; (S) GBRTRLIN201, phase contrast; (T) GBRTRLIN201, DIC; (U) 21-75, phase contrast; (V) 21-75, DIC; (W) 20-70, phase contrast; (X) 20-70, DIC; (Y) 21-74, phase contrast; (Z) 21-74, DIC. 


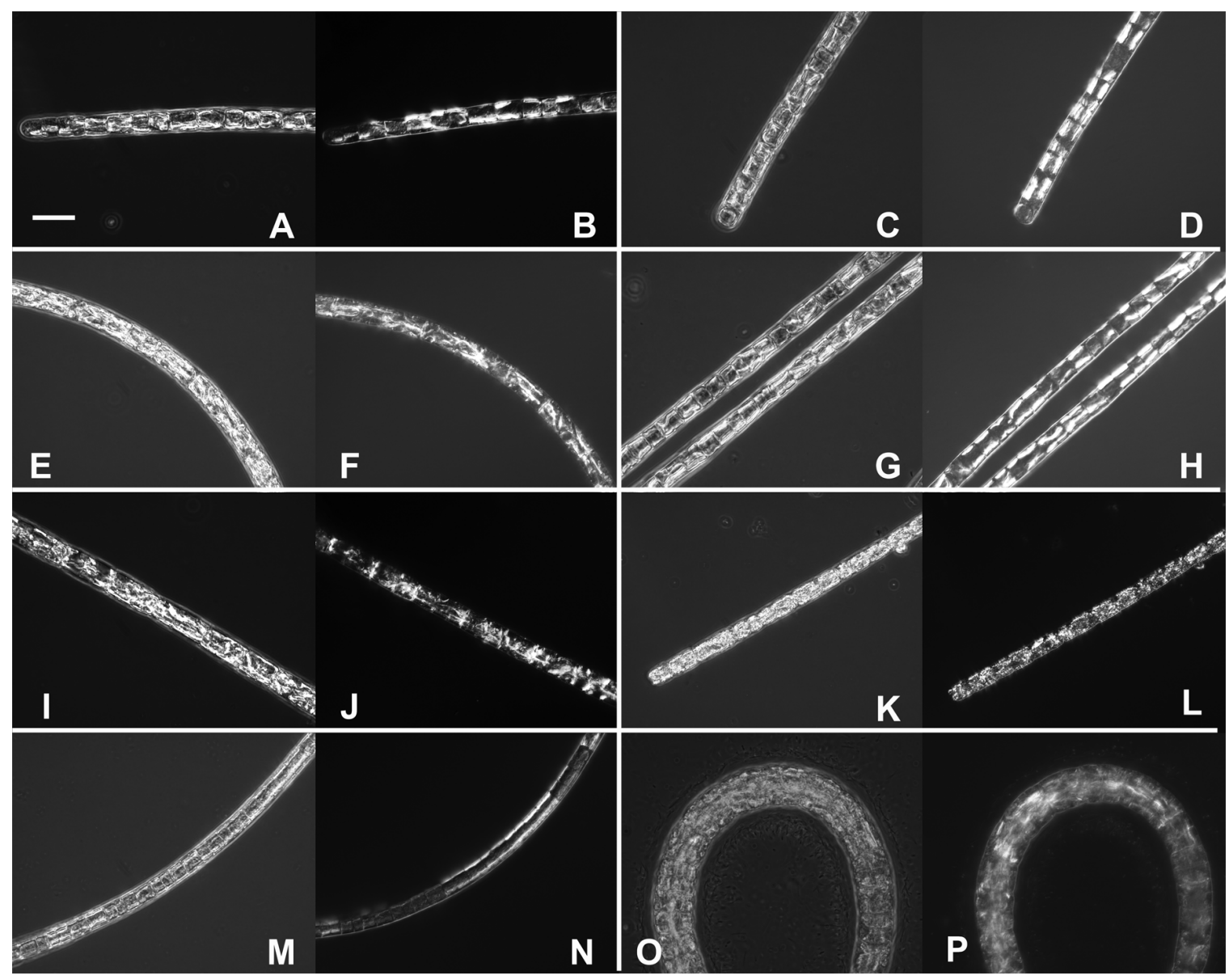

Figure 2-16: Trichodesmium clade II micrographs taken using phase contrast and differential interference contrast (DIC): T. thiebautii (A - J), T. tenue $(\mathrm{K}-\mathrm{N})$, and T. pelagicum (O - P). The white bar in (A) represents $20 \mu \mathrm{m}$. (A) II-3, phase contrast; (B) II-3, DIC; (C) VI-1, phase contrast; (D) VI-1, DIC; (E) St. 1-2, phase contrast; (F) St. 1-2, DIC; (G) St. 3-4, phase contrast; (H) St. 3-4, DIC; (I) St. 5-1JW, phase contrast; (J) St. 5-1JW, DIC; (K) tenue, phase contrast; (L) tenue, DIC; (M) H9-4, phase contrast; (N) H9-4, DIC; (O) 20-71, phase contrast; (P) 20-71, DIC. 
Absorption spectra of phycobiliproteins. The major phycobiliprotein in all the measured strains of Trichodesmium was PE with the ratio of PUB:PEB varying between species clusters. All strains had a PE:PC ratio > 1 and the AP peak was not detectable (Table 2.4, Fig. 2-17).

In clade I, all strains of T. erythraeum and T. contortum had a PUB:PEB ratio < 1 (0.79 - 0.92). PE:PC ratios ranged from 1.75 - 3.64 for T. erythraeum and 5.11 - 5.19 for T. contortum (Table 2.4). In this clade, the wavelength of maximum absortion $\left(\lambda_{\max }\right)$ for PUB was 497.0 - $498.0 \mathrm{~nm}, \lambda_{\max }$ for PEB was 543.0 - $546.5 \mathrm{~nm}$, and $\lambda_{\max }$ for PC was 613.5 - $620.0 \mathrm{~nm}$ (Table 2.4, Fig. 2-17 A). Some strains in this clade showed a shoulder on the PEB peak around 555 nm: T. erythraeum strains IMS101, K-02 \#2, K-04 \#20, XIII-13, St. 5-1A, GBRTRLI101, GBRTRLIN201, and 21-75 and T. contortum 20-70 (Fig 2-17 A). T. erythraeum strains GBRTRLI101 and GBRTRLIN201 had absorption peaks at 436.0 and $671.0 \mathrm{~nm}$, indicating small amounts of chlorophyll contamination (Fig. 2-17 A).

In clade II, all strains of T. thiebautii and T. tenue had a PUB:PEB ratio $>1(1.15$ - 1.54), PE:PC ratios ranged from 2.25 - 5.54, and T. tenue tenue did not have a discernible PC peak (Table 2.4, Fig. 2-17 B). In this clade, $\lambda_{\text {max }}$ for PUB ranged from 492.5 - 496.0 $\mathrm{nm}, \lambda_{\max }$ for PEB ranged from 540.0 - $555.0 \mathrm{~nm}$, and $\lambda_{\max }$ for PC ranged from 615.0 $620.0 \mathrm{~nm}$ (Table 2.4, Fig. 2-17 B).

\section{Discussion}

The phylogenetic trees show two distinct clades of cultured Trichodesmium, and within each clade are consistent subgroups. In clade I, T. erythraeum strains St. 5-1A, St. 6-1, St. 6-2, and St. 6-5 group together and T. contortum strains 20-70 and 21-74 group together. In clade II, strains tenue and H9-4 cluster together and T. thiebautii strains St. 1-2 and St. 5-1JW cluster with $T$. hildebrandtii. In general, the subgroups are morphologically similar to each other in size and gas vesicle distribution. The exception to this is the tenue/H9-4 subgroup. 

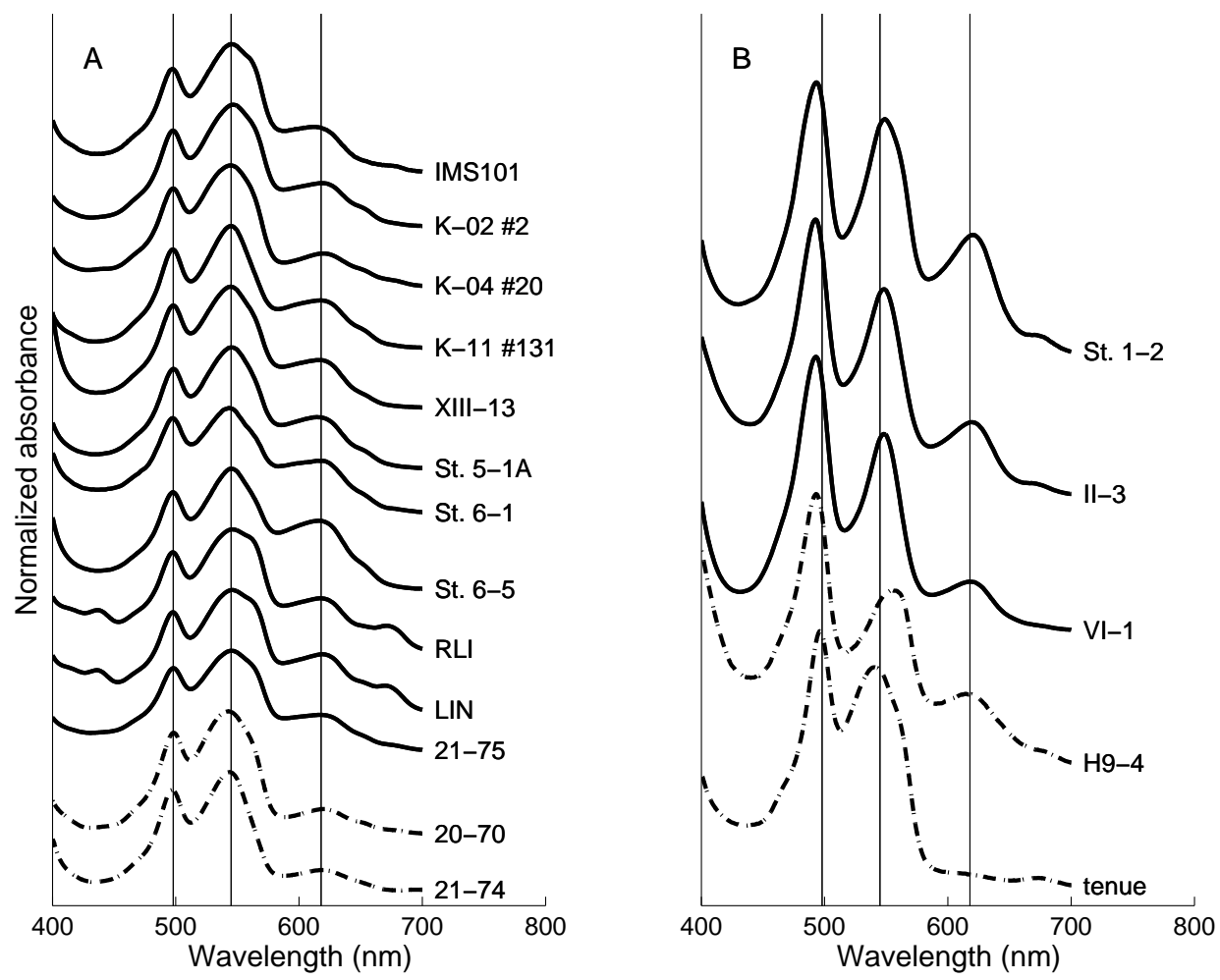

Figure 2-17: Absorption spectra of phycobiliproteins from cultured strains of Trichodesmium. Absorbance is normalized to the maximum absorbance. Graphs are offset vertically to show details. (A) Clade I spectra with PUB:PEB $<1$. Solid lines indicate $T$. erythraeum and dashed lines indicate T. contortum. (B) Clade II spectra with PUB:PEB $>1$. Solid lines indicate T. thiebautii and dashed lines indicate T. tenue. Vertical lines denote typical wavelengths of absorption peaks for PUB (498 nm), PEB (545 nm), and PC (618 nm).

Genotypes of Trichodesmium are circumtropical. The North Atlantic strain T. erythraeum IMS101 is more similar to strains from the South Pacific such as GBRTRLIN201 and K-02 \#2 than it is to other North Atlantic strains such as St. 6-1. Likewise, the Indian Ocean strain T. tenue tenue is most similar to the North Pacific strain H9-4.

The genetic grouping of species differs greatly from classical groupings based on morphology, in which T. tenue was clustered with T. erythraeum, and T. contortum was clustered with T. thiebautii (Janson et al., 1995). Our culture collection does not encom- 
pass the full range of Trichodesmium diversity. Genotypes identified as T. contortum and T. tenue in Janson et al. (1999) and T. aureum and T. havanum (Lundgren et al., 2005) are not represented among our cultures. The Janson T. contortum and T. tenue formed a clade separate from our cultured strains. The contradiction in genotypes of T. contortum and $T$. tenue found between our study and Janson et al. (1999) may be due to different species being identified with the same name. T. contortum specimens in Janson et al. (1999) occurred in colonies and were "a pale, straw-like color." Ours was bright red, indicating high PEB content, and occurred as single filaments. The T. tenue colonies in Janson et al. (1999) were larger and darker red than usually seen in T. tenue and had peripheral gas vesicles. Our T. tenue tenue has dispersed gas vesicles. Culturing efforts must continue to incorporate this diversity, and classification systems must be updated and revised as new data is available (Anagnostidis and Komárek, 1988).

Morphological characteristics can aid in the identification of Trichodesmium spp., but they are not infallible. Gas vesicles are variable and may not be a good characteristic for identification. Cells of the same species may display both random distribution and peripheral arrangement of gas vesicles. Cell morphometrics have a broad range within a clade and overlap between clades. Even IMS101, the most widely-used cultural representative of T. erythraeum, was initially identified as T. thiebautii (Prufert-Bebout et al., 1993). Although T. contortum is genetically very similar to T. erythraeum, they look vastly different. Similarly, T. pelagicum is so morphologically different from T. thiebautii that until genetic work was introduced, it was considered a separate genus, Katagnymene (Lundgren et al., 2001; Orcutt et al., 2002; Lundgren et al., 2005).

Phycobiliprotein composition was consistent with the phylogeny of the two major clades of Trichodesmium. This result is unexpected since phycobiliproteins have not been reliable taxonomic markers. For example, PUB:PEB ratios in closely-related strains of Synechococcus ranged from 0.4 - 2.0 while unrelated strains had similar PUB:PEB ratios (Toledo et al., 1999). Trichodesmium strains in clade I were all brick red in color due to the prevalence of PEB (PUB:PEB $=0.79$ - 0.92). The spectra in this clade were 
highly similar. Some of the strains displayed a shoulder on the red side of the PEB peak. The two T. contortum strains were similar in that they had much less PC than the T. erythraeum strains, shown by the PE:PC ratio: $5.11-5.19$ compared to $1.75-3.64$ in $T$. erythraeum. The two T. contortum strains differed in that 20-70 had the PEB shoulder while 21-74 did not. Clade II varied in color and $\lambda_{\max }$ of the PE subunits, but they all had PUB as the dominant subunit of PE. With increasing PUB:PEB, cells become greener: T. tenue tenue is salmon pink (PUB:PEB $=1.15$ ), . thiebautii strains tend to be brown $(\mathrm{PUB}: \mathrm{PEB}=1.15-1.39)$, and Trichodesmium sp. H9-4 is sage green (PUB:PEB = 1.54). The $\lambda_{\max }$ for PUB (493.0 - $496.0 \mathrm{~nm}$ ) is blue-shifted for this clade compared to that of clade I (497.0 - $498.0 \mathrm{~nm})$. In clade II, the $\lambda_{\max }$ for PEB was red-shifted compared to that of clade I (548.0 - $549.0 \mathrm{~nm}$ compared to 543.0 - $546.5 \mathrm{~nm}$, respectively). Trichodesmium sp. H9-4 had the most red-shifted $\lambda_{\max }$ for PEB at $555 \mathrm{~nm}$ and T. tenue tenue had the most blue-shifted $\lambda_{\max }$ for PEB at $540.0 \mathrm{~nm}$, the shortest wavelength for all strains regardless of species.

The consistency of absorption spectra within monophyletic groups of Trichodesmium indicated that pigment composition may be used to aid in species identification where cell morphology may be ambiguous. In South Pacific field populations of Trichodesmium, the spectra of field T. erythraeum matched the absorption spectra presented here for clade I, and the spectrum of a $T$. thiebautii-like green colony resembled the spectra for clade II (Neveux et al., 2006). However, South Pacific T. thiebautii and T. pelagicum (Katagnymene) samples had lower PUB:PEB ratios than clade II cultured samples: 0.98 - 1.00 and 0.68, respectively, compared to 1.15 - 1.54 (Neveux et al., 2006). A Caribbean Sea population identified as a mixture of T. erythraeum and T. thiebautii had absorption spectra similar to that of clade II (Subramaniam et al., 1999). This study indicates that Trichodesmium with low PUB:PEB ratios belong to clade I while those with high PUB:PEB ratios belong to clade II, but further studies are required to understand the phenotypic plasticity within each clade.

Diverse PUB:PEB ratios point to the possibility of niche differentiation in Tri- 
chodesmium. In the South Pacific, green colonies of Trichodesmium with high PUB were found at 50 - 120m, deeper than the high PEB Trichodesmium collected (Neveux et al., 2006). PUB absorbs shorter wavelengths of light than PEB and hence is adapted for greater depths (Neveux et al., 2006). Conversely, Subramaniam et al. (1999) found that PUB:PEB increased from 1.7 at night to 2.1 at midmorning for Caribbean Trichodesmium specimens at $15 \mathrm{~m}$. The increase in PUB may downregulate the energy supply to photosystem II to prevent damage to the reaction center at high light (Subramaniam et al., 1999). Interpretation of the role of PUB in Trichodesmium ecology requires environmental and physiological context.

The green field colonies described in Neveux et al. (2006) may be closely related to our cultured strain H9-4. Orcutt et al. (2008) reported that the DNA fingerprint of the green colonies was identical to that of T. thiebautii. While the green color has been suggested to be an indication of senescence (Orcutt et al., 2008), the green field colonies were viable (Neveux and Tenório, 2008), and green appears to be the native color of H9-4.

H9-4 is a taxonomic enigma. Its cell morphology is most similar to that of $T$. thiebautii; its genetic markers most resemble T. tenue; it forms single trichomes, puffs, and tufts all in the same flask; and its color is like nothing else: green with a long $\lambda_{\max }$ for PEB $(555.0 \mathrm{~nm})$. The morphological characteristics of H9-4 and T. tenue tenue are divergent. H9-4 usually has peripheral gas vesicles while tenue's are randomly distributed, H9-4 cells are mostly square-shaped while tenue cells are much longer than they are wide, H9-4 cells are green while tenue cells are pink, and H9-4 has different PUB and PEB $\lambda_{\max }$ 's than tenue: $493.0 \mathrm{~nm}$ compared to $496.0 \mathrm{~nm}$ and $555.0 \mathrm{~nm}$ compared to $540.0 \mathrm{~nm}$, respectively. In spite of the morphological and pigmentation differences, I have classified H9-4 as T. tenue based on its genetic similarity to T. tenue tenue.

Using sequence data, I have been able to show that there are two distinct clades of Trichodesmium represented in our culture collection. Studying the metagenome of these two clades could help us to understand potentially important differences between 
them and to fine-tune the phylogenetic relationships of Trichodesmium. Finding a genetic basis for cell size and shape could help to shed light on the morphological diversity found within each clade. Future studies examining the temperature optima and chromatic adaptions of cultured strains could illuminate the possibilities for niche differentiation in Trichodesmium. The culture collection does not incorporate the full diversity of Trichodesmium found in field studies, so culturing efforts should continue.

Acknowledgements. I would like to thank J. B. Waterbury for providing the vast majority of cultured strains in this work and his advice on microscopy, F. X. Fu for providing the Great Barrier Reef strains via E. A. Webb, F. Valois and J. Confalone for their assistance in the phycobiliprotein (PBP) analysis, S. C. Doney for his advice on data presentation, E. Webb for his advice on extracting Trichodesmium DNA and polymerase chain reaction (PCR), E. Lorch for her assistance in doing a few of the PCR's, the T. Shank lab especially R. Waller for her advice and for the use of their space and thermocycler for the sequencing reactions, the Keck facility at the Marine Biological Laboratory and Northwoods DNA, Inc. for sequence data, P. D. Chappell for her advice on analyzing sequence data, and B. G. Hall for his guide on phylogenetic trees (Hall, 2004). This research was supported by the Woods Hole Oceanographic Institution (WHOI) Ocean Life Institute (OLI) grant to J. Waterbury and the Center for Microbial Oceanography Research and Education (C-MORE), an NSF Science and Technology Center (EF-0424599). 


\title{
CHAPTER 3
}

\section{Development of a quantitative polymerase chain reaction (qPCR) assay to distinguish the two clades of Trichodesmium}

\begin{abstract}
To understand the role Trichodesmium has in the global nitrogen cycle, we must understand the distribution of Trichodesmium spp. Previous applications of real-time quantitative polymerase chain reaction (qPCR) to Trichodesmium populations have been at the genus level. Investigating ecological differences among species of Trichodesmium requires an assay with higher resolution, but designing a species-specific assay is difficult due to the high degree of genetic similarity among Trichodesmium spp. There are two distinct clades of Trichodesmium; clade I contains Trichodesmium erythraeum and Trichodesmium contortum, and clade II contains Trichodesmium thiebautii, Trichodesmium tenue, Trichodesmium hildebrandtii, and Trichodesmium pelagicum. In this study, I developed a qPCR method that is able to distinguish between the two major clades of Trichodesmium, targeting the heterocyst differentiation gene hetR. Trichodesmium spp. produce compounds which can inhibit PCR, making quantitative assays troublesome, so I tested a variety of DNA extraction methods to minimize inhibition. This method was tested in pure and mixed cultures of Trichodesmium for specificity and efficiency, and can be applied to field populations to identify and enumerate the two clades.
\end{abstract}




\section{Introduction}

Cyanobacteria of the genus Trichodesmium contribute significant amounts of nitrogen $(\mathrm{N})$ to tropical and subtropical oligotrophic oceans through $\mathrm{N}_{2}$ fixation (Capone et al., 1997). In order to constrain inputs of $\mathrm{N}$ to marine ecosystems, the spatial and temporal distributions of Trichodesmium spp. must be understood. Cell counts, specifically trichome (filament) and colony counts, can be effective (Letelier and Karl, 1996; Post et al., 2002; Tyrrell et al., 2003). In the Gulf of Aqaba, Red Sea, fusiform "tuft" colonies were found near the surface while spherical "puff" colonies were found in the lower half of the photic zone (Post et al., 2002). In the eastern Atlantic, Trichodesmium was found at highest trichome and colony densities between $0-15^{\circ} \mathrm{N}$, with a complete absence south of $30^{\circ} \mathrm{S}$ (Tyrrell et al., 2003). Trichomes and colonies vary in size and their counts may not represent true Trichodesmium biomass. Cell counts can also be laborious, and identifying trichomes to species based on morphology can be confounding (Chapt. 2).

Trichodesmium biomass was long-considered to be concentrated in the upper 50 m of the water column (Letelier and Karl, 1996; Carpenter et al., 2004). Collection of Trichodesmium via net tows is limited to surface regions, as long hauls can damage the fragile trichomes. A non-invasive study of Trichodesmium distributions using a video plankton recorder (VPR) reported high concentrations of Trichodesmium colonies as deep as $130 \mathrm{~m}$ (Davis and McGillicuddy, 2006). The VPR also revealed disparate depth distributions between colony morphologies; tufts were concentrated near the surface while puffs were distributed throughout the upper water column (Davis and McGillicuddy, 2006). The VPR provides an important alternative to net tows and cell counts. However, the VPR is unable to distinguish species of Trichodesmium and does not detect single trichomes, which can make up the majority of biomass (Letelier and Karl, 1996).

Real-time quantitative polymerase chain reaction (qPCR) has been used to investigate population dynamics of marine cyanobacteria on spatial and temporal scales. Ecotypes of Prochlorococcus have been resolved using sequences of the $16 \mathrm{~S}-23 \mathrm{~S}$ internal 
transcribed spacer (ITS) (Ahlgren et al., 2006; Zinser et al., 2006; Johnson et al., 2006). Diazotrophs including heterocystous Richelia spp., unicellular Crocosphaera spp, unicellular "group A," and non-heterocystous Trichodesmium spp. have been enumerated by targeting nifH, one of the genes encoding the $\mathrm{N}_{2}$-fixing enzyme nitrogenase (Church et al., 2005; Foster et al., 2007; Goebel et al., 2007).

The nifH qPCR assay targets the genus Trichodesmium as a whole. Species of Trichodesmium are highly similar genetically, making species-specific primers difficult to design. The species of Trichodesmium fall into two clades. Clade I includes Trichodesmium erythraeum and Trichodesmium contortum, and clade II includes Trichodesmium thiebautii, Trichodesmium tenue, Trichodesmium pelagicum (formerly Katagnymene spp.), and Trichodesmium hildebrandtii (Chapt. 2). In order to elucidate important ecological differences between the clades, a qPCR assay must target each clade individually.

The purpose of this study was to develop a SYBR green qPCR method to enumerate and identify the two major clades of Trichodesmium. SYBR green fluoresces only when bound to double-stranded DNA. As PCR progresses, the amplicon increases exponentially, and the SYBR green signal increases. Artifacts such as primer-dimers, secondary structures, and non-specific products can increase the SYBR green signal and overestimate the quantity of the region of interest. Melting temperature $\left(\mathrm{T}_{m}\right)$ of a PCR product is affected by its length as well as its sequence, so non-specific products can be detected using a melt curve analysis. For an effective assay, amplicons should be $<200$ bp, primers should not bind to themselves or each other to form dimers, the amplicon should not have secondary structures such as hairpins, and the primers should not amplify non-specific products (Templeton and Claas, 2003). I used sequences of the heterocyst differentiation protein gene hetR from Chapt. 2 to design primers targeting each clade of Trichodesmium. I tested the qPCR primers with serial dilutions and mixtures of Trichodesmium cultures using a variety of DNA extraction methods to minimize PCR inhibition. The method is able to accurately enumerate and identify the two clades of Trichodesmium in mixed cultures. 
Table 3.1: Sequences of clade-specific primers used in qPCR

\begin{tabular}{lll}
\hline Clade & Primer & Sequence (5' to 3') \\
\hline I & hetR-TeryF & GCAGGAAGTAGAAGAAGCAC \\
& hetR-TeryR & TTACCAGGAAGGCGAGAAC \\
\hline II & hetR-ThiebF & TTCCTGGTGGTAATCTGACTAC \\
& hetR-ThiebR & CTTCCTGAGAACGCCTATGG \\
\hline
\end{tabular}

\section{Methods}

Primer design and preparation of cloned qPCR standards. Sequences of the heterocyst differentiation gene hetR from cultured strains of Trichodesmium (Chapt. 2) were used to design primers for SYBR green using AlleleID software (PREMIER Biosoft International). Primers targeted different hetR sequences within each clade of Trichodesmium while excluding the other clade as well as marine filamentous cyanobacteria Richelia (GenBank accession numbers DQ366915 and DQ366914) and Calothrix (GenBank accession number DQ366916) (Benson et al., 2005; Foster and Zehr, 2006). Forward and reverse primers for each clade are listed in Table 3.1 and amplify different but overlapping regions of hetR for each clade. AlleleID was also used to design SYBR green primers targeting ITS. The primer pairs were tested in silico using MacVector on sequences from all six species of Trichodesmium.

All PCR optimization was performed on an iCycler thermal cycler (BioRad) with $25 \mu \mathrm{L}$ reactions. The primers were optimized using a temperature gradient to find an annealing temperature that was low enough for efficient PCR in the targeted clade but high enough to exclude the non-targeted clade. PCR was conducted using the MasterTaq Kit (Eppendorf, 5 Prime) to get a $140 \mathrm{bp}$ fragment for Clade I and a $138 \mathrm{bp}$ fragment for Clade II. Reactions consisted of $1 \times$ Taq Master PCR Enhancer, $1 \times$ Taq Buffer, $200 \mu \mathrm{M}$ dNTPs, $0.5 \mu \mathrm{M}$ each of primers hetR-TeryF and hetR-TeryR for clade I and primers hetRThiebF and hetR-ThiebR for clade II (Table 3.1), 1.25 U Taq DNA polymerase, and $4 \mathrm{ng}$ genomic DNA from cultured strains. Cycling conditions were $94^{\circ} \mathrm{C}$ for $2 \mathrm{~min} ; 30$ cycles 
of $94^{\circ} \mathrm{C}$ for $30 \mathrm{~s}, 62^{\circ} \mathrm{C}$ (clade I) or $61^{\circ} \mathrm{C}$ (clade II) for $30 \mathrm{~s}$, and $72^{\circ} \mathrm{C}$ for $30 \mathrm{~s}$; and one cycle of $72^{\circ} \mathrm{C}$ for $5 \mathrm{~min}$. Both primer sets were tested using DNA from T. erythraeum strains IMS101, St. 5-1A, St. 6-1, XII-13, and K-04 \#20; T. contortum strains 21-74 and 20-70; T. thiebautii strains II-3, VI-1, St. 1-2, St. 3-4, and St. 5-1JW; T. tenue strains tenue and H9-4; and T. pelagicum 20-71 (Chapt. 2). Five $\mu \mathrm{L}$ of each PCR product was separated on a $2 \%$ agarose gel, stained with ethidium bromide, and photographed using a Gel Logic 200 Imaging System and Molecular Imaging Software (Kodak). The clade I assay was optimized to amplify only strains of clade I, and the clade II assay was optimized to amplify only strains of clade II.

Standards for qPCR were derived from cloned PCR products for each clade. To provide PCR products for cloning, T. erythraeum IMS101 was amplified using the clade I protocol and T. thiebautii II-3 was amplified using the clade II protocol as described above with the modification of 25 cycles instead of 30 . Products were cloned and linearized as described in Zinser et al. (2006). Briefly, the products were cloned directly into the pCR4-TOPO vector and introduced into Escherichia coli TOP10 cells using the TOPO-TA cloning kit for sequencing (Invitrogen) following the manufacturer's directions. Clones were screened using LBKan50 (50 $\mu \mathrm{g} \mathrm{mL}^{-1}$ kanamycin in Luria-Bertani medium). Plasmids from the E. coli cells were purified using the QIAprep Spin Miniprep kit (Qiagen) following manufacturer's instructions and stored at $-80^{\circ} \mathrm{C}$. To linearize the purified plasmids, digestion was performed using a PstI restriction enzyme kit (New England Biolabs) in $30 \mu \mathrm{L}$ reactions with $1 \times$ buffer $3,1 \times$ bovine serum albumin (BSA), 2 $\mu \mathrm{L}$ PstI, and $3 \mu \mathrm{L}$ plasmid. Reactions were incubated at $37^{\circ} \mathrm{C}$ for $3 \mathrm{~h}$ and products were visualized on a $1 \%$ agarose gel to confirm a single restriction site. Linearized plasmids were either sequenced as described in Chapt. 2 by the Keck Facility at the Josephine Bay Paul Center, Marine Biological Laboratory, Woods Hole, MA, or purified plasmids were sent to Agencourt Bioscience Corporation, Beverly, MA. Plasmids whose sequences were identical to those of T. erythraeum IMS101 and T. thiebautii II-3 were serially diluted (10-fold), quantitated using the Quant-iT PicoGreen kit (Molecular Probes/Invitrogen) 
following manufacturer's instructions, and stored to be used as qPCR standards. Copies of $h e t R$ volume ${ }^{-1}$ for each standard was calculated using the molecular weight of the plasmid plus the insert. All products were stored at $-80^{\circ} \mathrm{C}$.

Serial dilutions and cell counts of Trichodesmium cultures. Cultures of T. erythraeum IMS101, T. contortum 21-74, T. thiebautii II-3, T. tenue tenue, and T. tenue H9-4 were grown as described in Chapt. 2. For dilution series, cultures were diluted 10-fold up to 10,000-fold. For qPCR, 5 - $100 \mathrm{~mL}$ of diluted cultures were vacuum-filtered on $47 \mathrm{~mm}$, $5 \mu \mathrm{m}$ polycarbonate filters (Millipore) using a glass funnel and fritted base (Millipore) and filters were stored in liquid $\mathrm{N}_{2}$ in $1.8 \mathrm{~mL}$ cryo tubes (Nunc). Dilutions of T. erythraeum IMS101 were mixed with dilutions of T. tenue tenue and dilutions of $T$. contortum were mixed with dilutions of $T$. thiebautii II-3 to test the ability of the qPCR assay to distinguish between the two clades. For cell counts, 1 - $300 \mathrm{~mL}$ diluted cultures were vacuum-filtered on $25 \mathrm{~mm}, 5 \mu \mathrm{m}$ polycarbonate filters (Millipore) using a glass funnel and fritted base (Millipore) and fixed with $1 \mathrm{~mL} \mathrm{10 \%} \mathrm{buffered} \mathrm{formalin} \mathrm{(Surgipath)}$ for $2 \mathrm{~min}$. Fixed filters were mounted on a glass microscope slide using immersion oil, covered with a $25 \mathrm{~mm}$ square glass cover slip, and frozen at $-20^{\circ} \mathrm{C}$ until they could be viewed. Slides were viewed using epifluorescence (rhodamine filter) with a Zeiss Axioplan 2 microscope under either a $5 \times$ or a $10 \times$ Plan-Neoflaur objective lens (Fig. 31A). At least 20 micrographs per slide were taken using a Zeiss Axiocam HRC digital camera and Axiovision 4.6.3 software. Cells were counted by taking the total filament length and dividing by an average cell length. Total filament lengths were measured using an automated image-processing script in MATLAB (MathWorks) using scripts from Kovesi (1999). In the automated image-processing, micrographs were filtered to reduce noise using a threshold value, phase congruency, or a fast Fourier transform (FFT), and then the edges were detected by hysteresis thresholding (Kovesi, 1999). The result is a binary image (Fig. 3-1B). The area of the filaments was calculated as the total number of white pixels in the binary image and total filament length was calculated by di- 


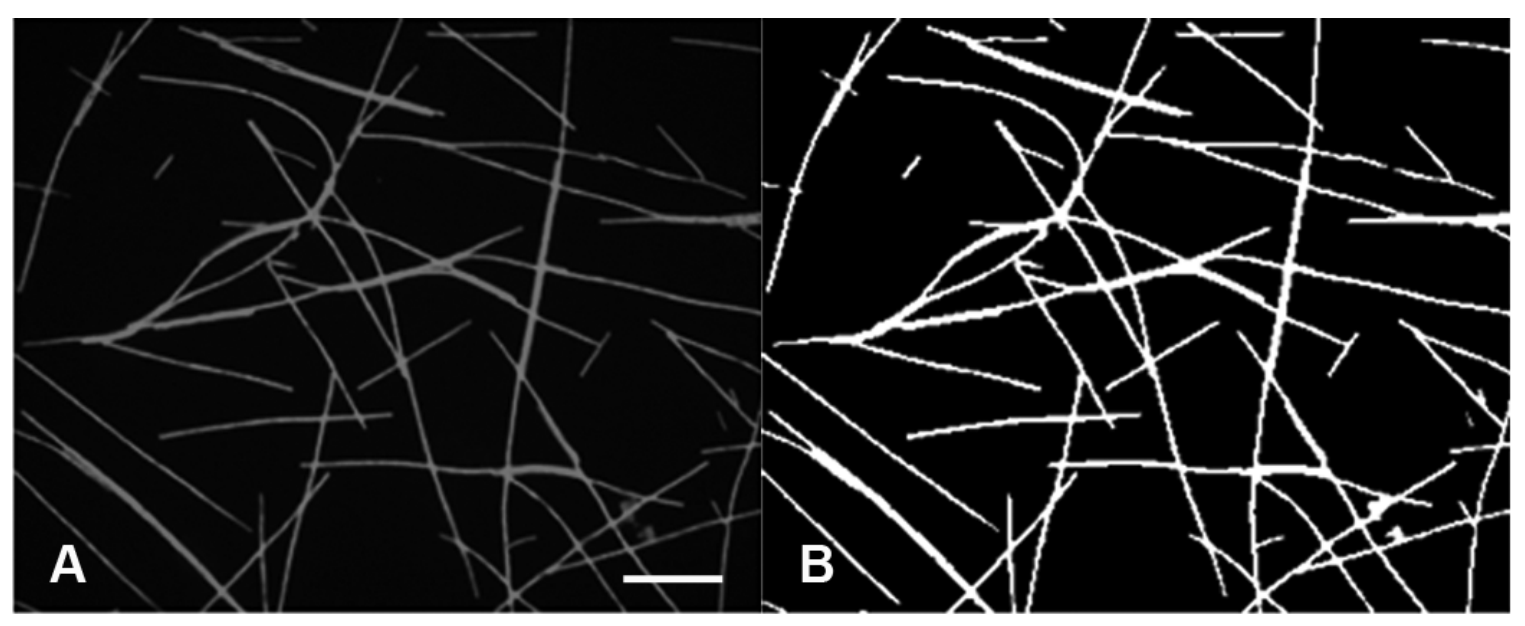

Figure 3-1: Image processing for automated cell counts. (A) Greyscale epifluorescence image of T. thiebautii II-3. (B) Filtered, binary image of (A). Cell counts are calculated from the white area in the processed image. White bar in (A) represents $200 \mu \mathrm{m}$.

viding the area by the width of a filament in the binary image. The automated counting method was validated by manually measuring filament lengths in 98 micrographs from $T$. erythraeum IMS101 and T. thiebautii II-3 using ImageJ software (National Institutes of Health, http://rsb.info.nih.gov/ij/).

DNA extraction. Due to problems with PCR inhibition, a variety of DNA extraction protocols were tested. Filters were aseptically transfered to a $2 \mathrm{~mL}$ bead beating tube (BioSpecs or MoBio) with two glass $6.4 \mathrm{~mm}$ beads (BioSpecs), four stainless steel beads (MoBio), or garnet beats (MoBio). To shred the filter and homogenize the samples, lysis buffer was added as described below and tubes were beat on high using a Mini-Beadbeater8 (BioSpecs) for two min, iced for one min, beat for two min, iced for one min, and beat for one min. All extracts and bead tubes with filters and lysates were stored at $-20^{\circ} \mathrm{C}$.

Hot tris method. Samples were extracted according to Zinser et al. (2006) with some modifications. Filters were transfered to bead tubes with glass beads and $650 \mu \mathrm{L}$ $\mathrm{pH}$ 8.0, $10 \mathrm{mM}$ Tris- $\mathrm{HCl}$ and beat as described above. Avoiding the filter, $250 \mu \mathrm{L}$ were transfered to $1.5 \mathrm{~mL}$ microcentrifuge tubes and incubated at $95^{\circ} \mathrm{C}$ for $15 \mathrm{~min}$. 
Qiagen tissue method. Samples were extracted using the DNeasy Blood \& Tissue Kit (Qiagen) following the protocol for animal tissue. Filters were transfered to bead tubes with glass beads and $650 \mu \mathrm{L}$ buffer ATL, and beat as described above. $250 \mu \mathrm{L}$ lysate were transfered to a new $1.5 \mathrm{~mL}$ microcentrifuge tube and extracted following the manufacturer's instructions. DNA was eluted from the Mini Spin column sequentially with 150 $\mu \mathrm{L}$ and $100 \mu \mathrm{L}$ buffer $\mathrm{AE}$, combining the eluates.

Qiagen plant methods. Samples were extracted using the DNeasy Plant Mini Kit and the high-throughput DNeasy 96 Plant Kit (Qiagen). Filters were transfered to bead tubes with glass beads and $650 \mu \mathrm{L}$ buffer AP1, and beat as described above. $250 \mu \mathrm{L}$ lysate were transfered to a new $1.5 \mathrm{~mL}$ microcentrifuge tube or the DNeasy microtubes and extracted following the manufacturer's instructions. DNA was eluted from the Mini Spin column with $150 \mu \mathrm{L}$ and $100 \mu \mathrm{L}$ buffer AE or from the DNeasy 96 plate twice with 100 $\mu \mathrm{L}$, combining the eluates.

MoBio plant method. Samples were extracted using the Power Plant Kit (MoBio). Filters were transfered to bead tubes with stainless steel beads and $650 \mu \mathrm{L}$ Power Plant Bead Solution, and beat as described above. $400 \mu \mathrm{L}$ lysate were transfered to a new 2.0 $\mathrm{mL}$ microcentrifuge tube and extracted following the manufacturer's instructions. DNA was eluted twice from the spin filter with $50 \mu \mathrm{L}$ buffer PB6, combining the eluates.

MoBio soil method. Samples were extracted using the Soil Kit (MoBio). Filters were transfered to bead tubes with garnet beads and $750 \mu \mathrm{L}$ lysis buffer, and beat as described above. $250 \mu \mathrm{L}$ lysate were transfered to a new $2.0 \mathrm{~mL}$ microcentrifuge tube and extracted following the manufacturer's instructions. DNA was eluted twice from the spin filter with $50 \mu \mathrm{L}$ buffer $\mathrm{C} 6$, combining the eluates.

Xanthogenate method. Samples were extracted using the xanthogenate-SDS method described by Tillett and Neilan (2000). Filters were transfered to bead tubes with glass beads and $650 \mu \mathrm{L}$ TER, beat as described above, and extracted (Tillett and Neilan, 2000). DNA pellets were washed three times with $75 \%$ ethanol, air-dried, and re-suspended in $200 \mu \mathrm{L}$ sterile $18.2 \mathrm{M} \Omega$ water. 


\section{Quantitative polymerase chain reaction (qPCR).}

iCycler method. Reactions were performed in an iCycler (BioRad) with $25 \mu \mathrm{L}$ reactions either in 8-strip 0.2 mL PCR tubes (Fisher) with optically clear flat cap strips (BioRad) or white 96-well plates (ABgene) with optically clear Microseal 'B' film (BioRad). Each reaction contained $1 \times$ iQ SYBR Green Supermix (BioRad), $0.5 \mu \mathrm{M}$ each of either primers hetR-TeryF and hetR-TeryR for clade I and primers hetR-ThiebF and hetR-ThiebR for clade II (Table 3.1), and $10 \mu \mathrm{L}$ template. Cycling conditions were one cycle at $95^{\circ} \mathrm{C}$ for 3 min, 40 cycles of $95^{\circ} \mathrm{C}$ for $30 \mathrm{~s}, 62^{\circ} \mathrm{C}$ for 30 s for clade I or $63^{\circ} \mathrm{C}$ for 30 s for clade II, and an additional step for clade II of $78^{\circ} \mathrm{C}$ for $15 \mathrm{~s}$ to melt primer dimers; followed by a melt curve analysis. Samples were run in triplicate and cloned standards were run in duplicate. The threshold cycle $\left(\mathrm{C}_{t}\right)$ was determined by the cycle at which the amplification curve crossed a threshold fluorescence value, calculated by the best fit to the standard curve of diluted cloned standards by the iCycler software. Sample concentration was calculated using the sample $\mathrm{C}_{t}$ and the standard curve. PCR efficiency $(E)$ was calculated as:

$$
E=\left(10^{-\frac{1}{m}}-1\right) \times 100 \%
$$

where $m$ is the slope of the linear regression of $\mathrm{C}_{t}$ to $\log _{10}$ (standard concentration).

LightCycler method. For a more high-throughput method, reactions were performed in a LightCycler-480 thermal cycler (Roche) with $20 \mu \mathrm{L}$ reactions in white 384-well plates sealed with optically clear sealing foil (Roche). Each reaction contained $1 \times$ LightCycler SYBR Green I Master (Roche), $0.25 \mu \mathrm{M}$ each of HPLC-purified primers hetR-TeryF and hetR-TeryR for clade I and primers hetR-ThiebF and hetR-ThiebR for clade II, and $2 \mu \mathrm{L}$ template. Master mix and DNA templates were loaded on the 384-well plate using a Precision 2000 liquid handler (Biotek Instruments). Cycling conditions were $95^{\circ} \mathrm{C}$ for 15 $\min ; 45$ cycles of $95^{\circ} \mathrm{C}$ for $20 \mathrm{~s}, 63^{\circ} \mathrm{C}$ for $30,72^{\circ} \mathrm{C}$ for $5 \mathrm{~s}$, and a primer dimer-melting step for clade II of $76^{\circ} \mathrm{C}$ for $10 \mathrm{~s}$; followed by a melt curve analysis. Samples and stan- 
dards were run in triplicate. Crossing point $\left(\mathrm{C}_{p}\right)$ was determined by the maximum second derivative method by the LightCycler software. Sample concentration was calculated using the sample $\mathrm{C}_{p}$ and the standard curve. PCR efficiency was calculated as in Equation 3.1 , where $m$ is the slope of the linear regression of $C_{p}$ to $\log _{10}$ (standard concentration).

\section{Results}

qPCR assay development. Cell counts done using the automated method agreed with counts done manually (Fig. 3-2). The slope of the linear fit of manually counted total filament length versus automated total filament length was $1.15 \pm 0.03$. The slope for the $\log _{10}-\log _{10}$ transformation was $0.98 \pm 0.02$, close enough to 1 to not transform the automated numbers before using them.
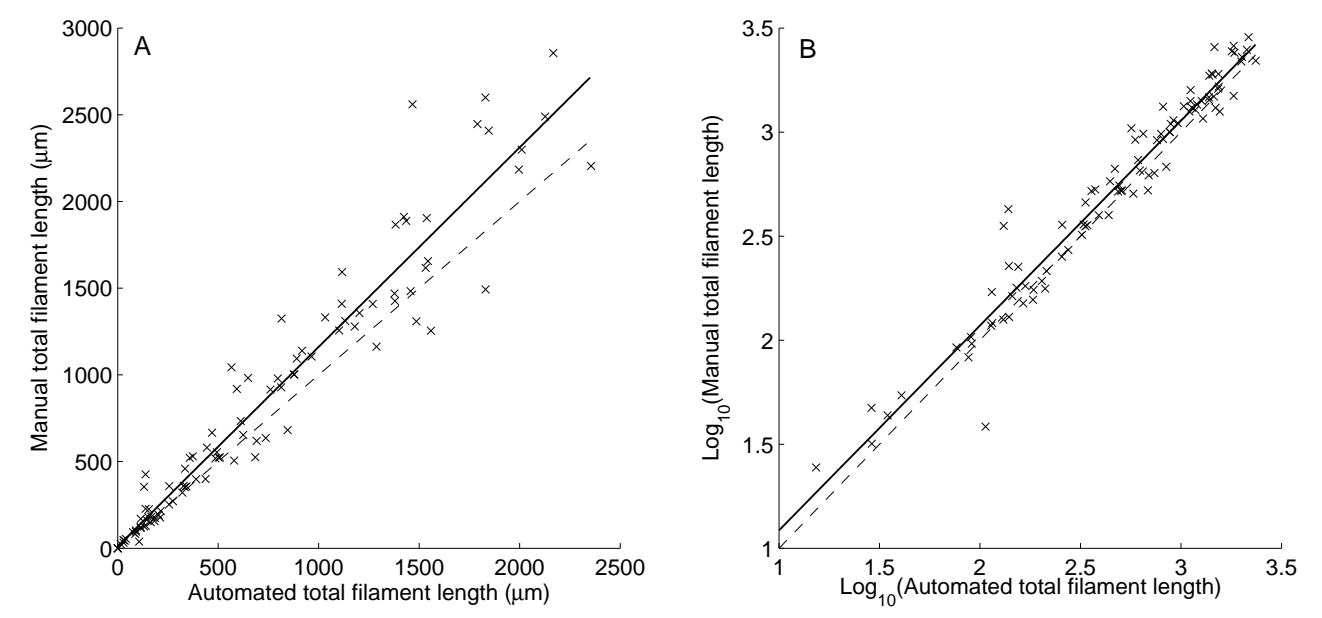

Figure 3-2: Comparison of the performance of the automated cell count method to the manually counted method. Solid line indicates a linear fit to the data. Dashed line indicates a 1:1 relationship. (A) Linear fit of manual total filament length versus automated filament lengths: $y=(1.15 \pm 0.03) \times x+10.9 \pm 20.5\left(\mathrm{R}^{2}=0.9263\right)$. (B) Linear fit of the $\log _{10}$ transformed data: $\log _{10}(y)=(0.98 \pm 0.02) \times \log _{10}(x)+0.10 \pm 0.01\left(\mathrm{R}^{2}=0.9501\right)$.

Representative standard curves for the qPCR assays are shown in Fig. 3-3. PCR efficiencies for the qPCR assays ranged from 93 - $102 \%$. Melting temperatures $\left(\mathrm{T}_{m}\right)$ were slightly higher for the iCycler method than for the LightCycler method (Fig. 3-4). 
$\mathrm{T}_{m}$ for the iCycler (LightCycler) clade I assay were $80.5^{\circ} \mathrm{C}\left(77.9^{\circ} \mathrm{C}\right)$ for the standards, $80.8^{\circ} \mathrm{C}\left(78.1^{\circ} \mathrm{C}\right)$ for T. erythraeum $\mathrm{IMS} 101$, and $79.9^{\circ} \mathrm{C}\left(78.3^{\circ} \mathrm{C}\right)$ for T. contortum 21 74 (Fig. 3-4A, C). The $\mathrm{T}_{m}$ for T. contortum 21-74 in the iCycler method was slightly lower than that of the standard while both of the cultured melt curves were nearly identical to the standard in the LightCycler method (Fig. 3-4A, C). For the clade II assay, iCycler (LightCycler) $\mathrm{T}_{m}$ 's were $79.6^{\circ} \mathrm{C}\left(77.04^{\circ} \mathrm{C}\right)$ for the standards, $79.6^{\circ} \mathrm{C}\left(77.3^{\circ} \mathrm{C}\right)$ for $T$. thiebautii II-3, $79.0^{\circ} \mathrm{C}\left(77.7^{\circ} \mathrm{C}\right)$ for $T$. tenue tenue, and $79.6^{\circ} \mathrm{C}\left(77.4^{\circ} \mathrm{C}\right)$ for T. tenue $\mathrm{H} 9-4$ (Fig. 3-4B, D). The $\mathrm{T}_{m}$ for T. tenue tenue was lower than the standard $\mathrm{T}_{m}$ for the iCycler method and higher than the standard for the LightCycler method while the melt curves for T. thiebautii II-3 and T. tenue H9-4 were identical to each other and nearly identical to the standard (Fig. 3-4B, D). The melt curves for the cultures in clade II showed evidence of primer dimers in the LightCycler method (Fig. 3-4D).

DNA extractions and serial dilutions. Serial dilutions of T. erythraeum IMS101 amplified well when extracted using the hot tris method described in Zinser et al. (2006) (data not shown). However, extracts of T. tenue H9-4 showed PCR inhibition (Fig. 3-5A). Alternative methods of DNA extraction were tested to determine a suitable method that did not show significant inhibition. The MoBio plant method yielded the highest amplification (Fig. 3-5). For high concentrations of T. tenue H9-4, the hot tris and Qiagen Tissue methods did not yield a PCR product (Fig. 3-5A). At all concentrations of H9-4, extractions from the Qiagen plant methods did not amplify as well as extractions from the MoBio plant method. The xanthogenate and MoBio soil extractions did not amplify as well as the MoBio plant extractions did for T. tenue H9-4 and T. erythraeum IMS101 (Fig. 35B).

Serial dilutions of Trichodesmium cultures extracted with the MoBio plant method did not result in a consistent relationship between cell counts and copies of hetR as measured by the qPCR assay (Fig. 3-6A-B). Linear fits of $\log _{10}$-transformed copies of hetR $\mathrm{mL}^{-1}$ to $\log _{10}$-transformed cells $\mathrm{mL}^{-1}$ were for $\operatorname{IMS101,} \log _{10}(y)=(1.40 \pm 0.02) \times$ 

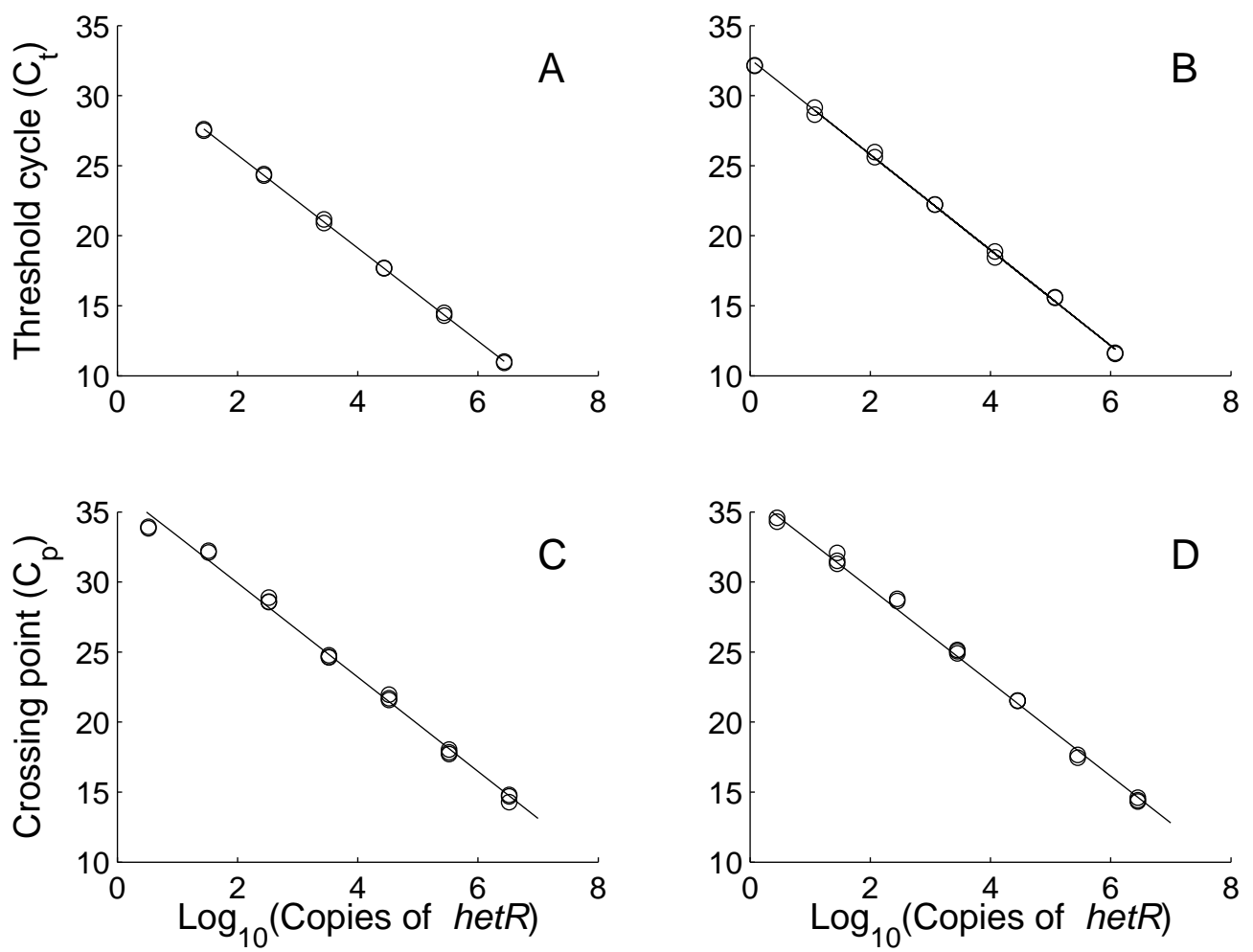

Figure 3-3: Standard curves from the qPCR assays. The y-axis represents either threshold cycle (iCycler method) or crossing point (LightCycler method) and the $\mathrm{x}$-axis represents $\log _{10}$ (copies of hetR). (A) Clade I, iCycler method $\left(E=100.1 \%, \mathrm{R}^{2}=1.000\right)$; (B) clade II, iCycler method $\left(E=96.6 \%, \mathrm{R}^{2}=0.999\right)$; $(\mathrm{C})$ clade I, LightCycler method $(E=$ $\left.98.5 \%, \mathrm{R}^{2}=0.994\right)$; (D) clade II, LightCycler method $\left(E=98.9 \%, \mathrm{R}^{2}=0.995\right)$.

$\log _{10}(x)-0.66 \pm 0.02\left(\mathrm{R}^{2}=0.9998\right.$, Fig. 3-6A); for 21-74, $\log _{10}(y)=(1.84 \pm 0.18) \times$ $\log _{10}(x)-1.44 \pm 0.15\left(\mathrm{R}^{2}=0.9907\right.$, Fig. 3-6A); for II-3, $\log _{10}(y)=(1.68 \pm 0.23) \times$ $\log _{10}(x)-1.48 \pm 0.18\left(\mathrm{R}^{2}=0.9823\right.$, Fig. 3-6B); for tenue, $\log _{10}(y)=(1.21 \pm 0.48) \times$ $\log _{10}(x)-0.00 \pm 0.39\left(\mathrm{R}^{2}=0.8627\right.$, Fig. 3-6B); and for H9-4, $\log _{10}(y)=(0.86 \pm 0.10) \times$ $\log _{10}(x)+1.11 \pm 0.08\left(\mathrm{R}^{2}=0.9873\right.$, Fig. 3-6B)

In mixed culture samples, the qPCR method was able to accurately measure the components of the mixture. Dilutions of T. erythraeum IMS101 were mixed with dilutions of T. tenue tenue and T. contortum 21-74 was mixed with T. thiebautii II-3. Fig. 3-7 

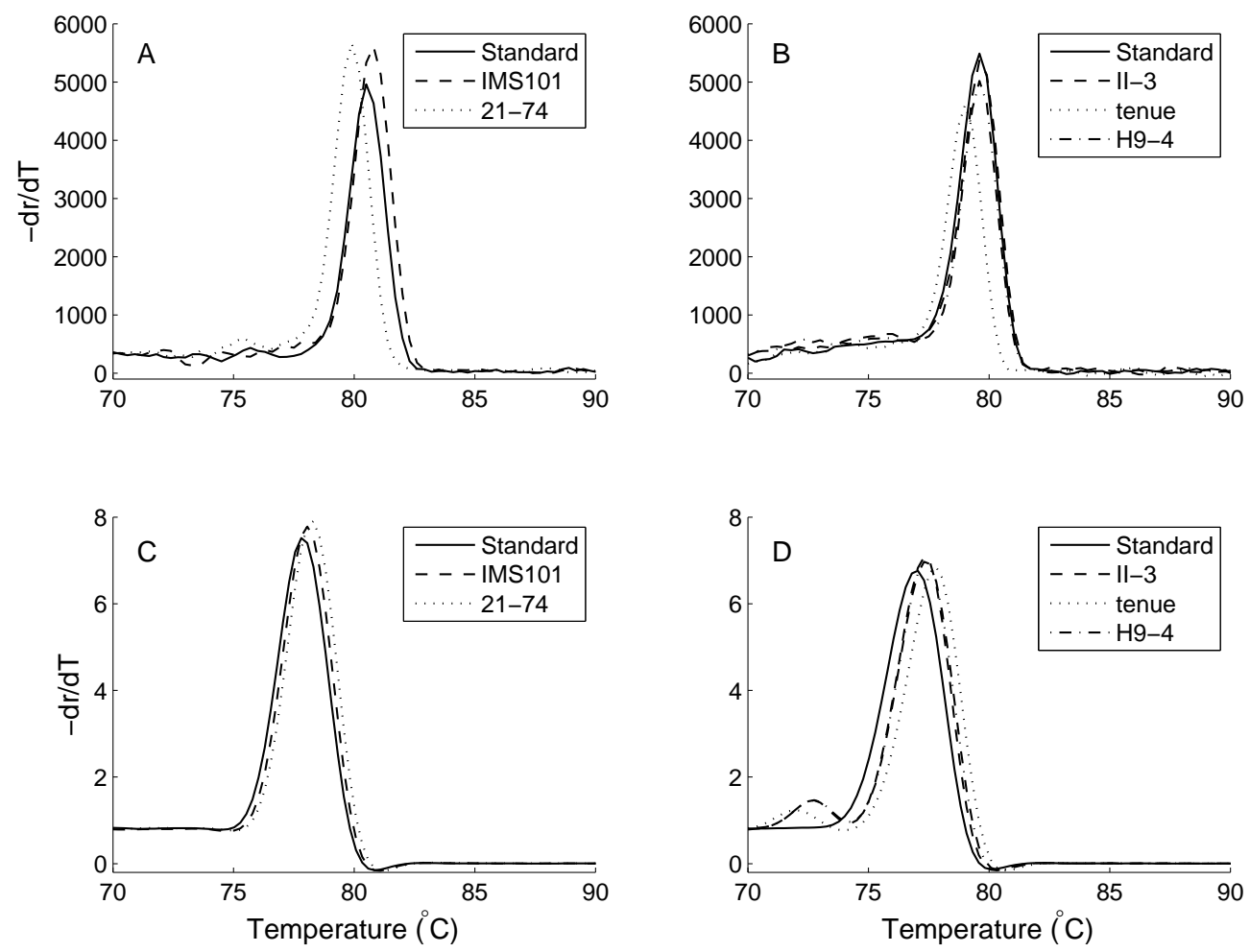

Figure 3-4: Melting curves from the qPCR assays. The y-axis represents the negative of the derivative of relative fluorescence unit with respect to temperature and the $\mathrm{x}$-axis represents temperature $\left({ }^{\circ} \mathrm{C}\right)$. (A) Clade I, iCycler method; (B) clade II, iCycler method; (C) clade I, LightCycler method; (D) clade II, LightCycler method.

shows a linear fit of the $\log _{10}$ transformed data of measured qPCR results versus expected qPCR results based on measurements of monocultures. The slope of the fit has a $95 \%$ confidence interval of $0.70-1.00\left(\mathrm{R}^{2}=0.9956\right)$, indicating a one-to-one relationship. 

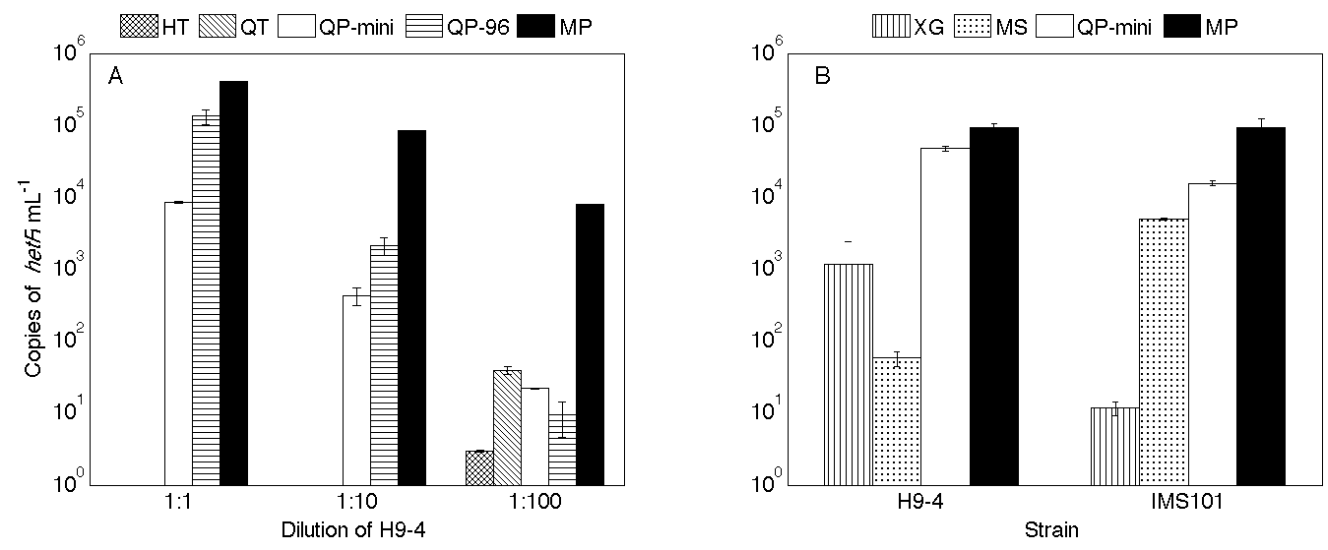

Figure 3-5: Comparison of DNA extraction methods for (A) serial dilutions of T. tenue H9-4, and for (B) replicate samples of T. tenue H9-4 and T. erythraeum IMS101. "HT" = hot tris (cross-hatched), "QT" = Qiagen DNeasy Tissue kit (diagonal stripes), "QP-mini" = Qiagen DNeasy Plant mini kit (white), "QP-96" = Qiagen DNeasy Plant-96 kit (horizontal stripes), "MP" = MoBio Plant kit (black), "XG" = xanthogenate (vertical stripes), and "MS" = MoBio Soil kit (dotted). Error bars represent the standard deviation of 2-3 replicates. 

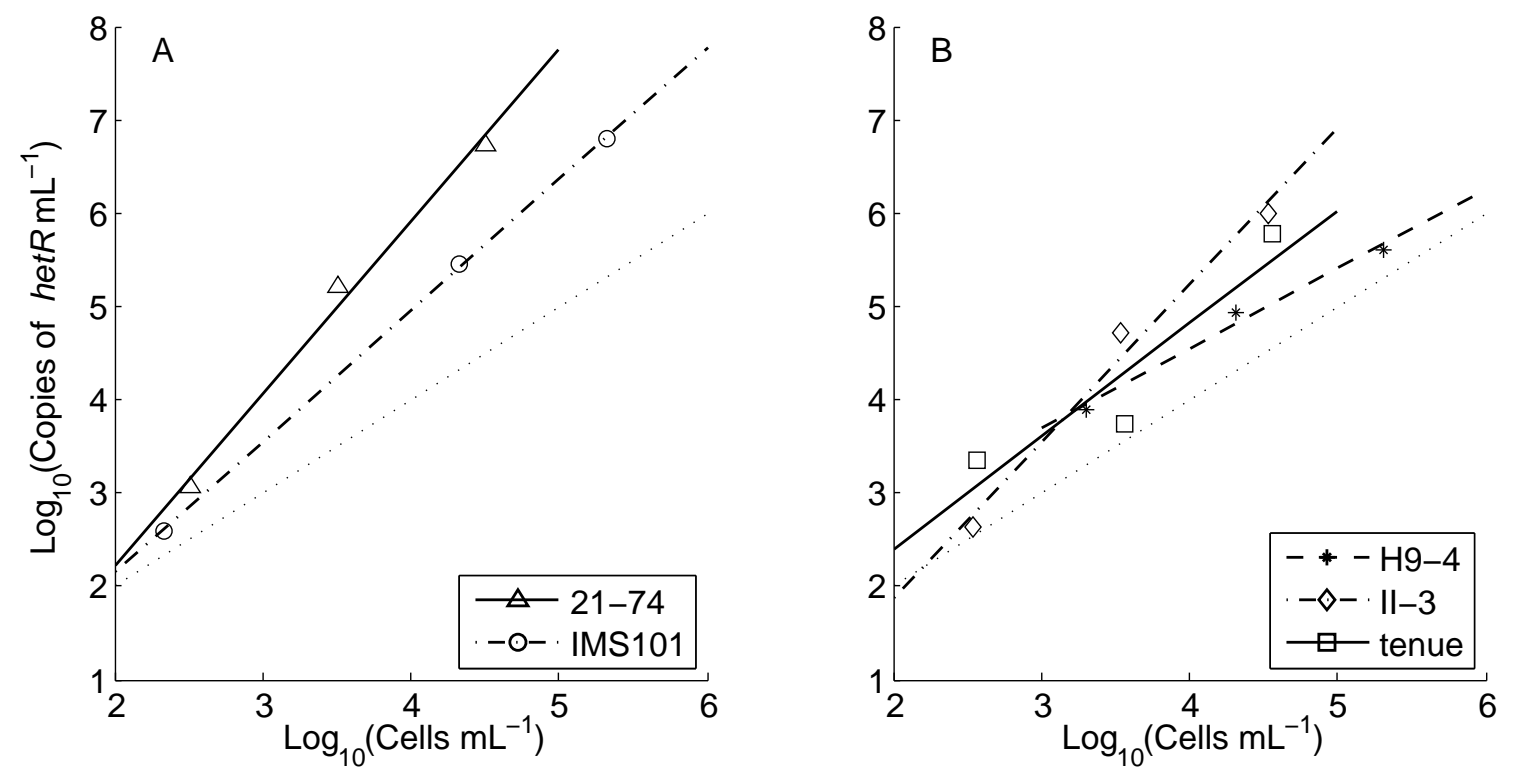

Figure 3-6: Linear fit of qPCR results from serial dilutions of Trichodesmium cultures using the LightCycler method in $\log _{10}$ (copies of het $R \mathrm{~mL}^{-1}$ ) versus $\log _{10}\left(\right.$ cells $\mathrm{mL}^{-1}$ ). Dotted lines indicate a 1:1 relationship. (A) Clade I strains: T. contortum 21-74 and $T$. erythraeum IMS101. (B) Clade II strains: T. tenue H9-4, T. thiebautii II-3, and T. tenue tenue.

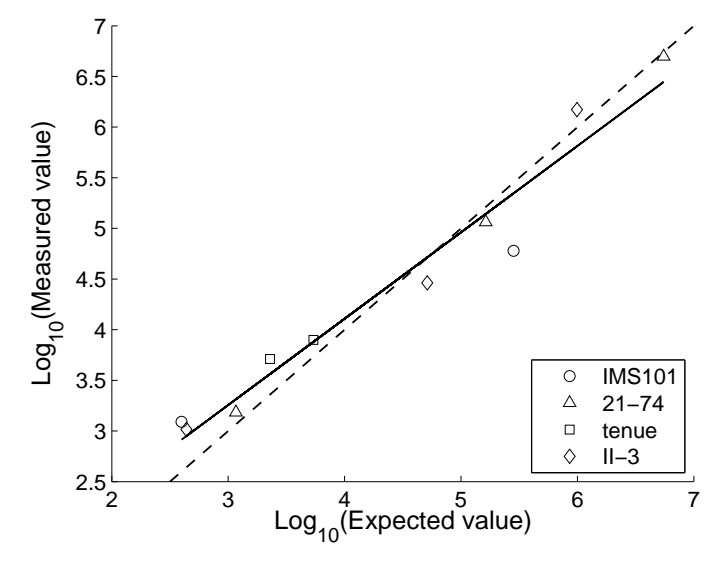

Figure 3-7: Mixed culture qPCR results. Measured results $\left(\log _{10}\right.$ (copies of hetR $\left.\mathrm{mL}^{-1}\right)$ ) were plotted against the expected results based on serial dilutions of monocultures $\left(\log _{10}\left(\right.\right.$ copies of het $\left.\left.R \mathrm{~mL}^{-1}\right)\right)$. Dilutions of T. contortum 21-74 were mixed with dilutions of T. thiebautii II-3, and dilutions of T. erythraeum IMS101 were mixed with dilutions of T. tenue tenue. The solid line is the linear regression fit through the $\log _{10}$ - transformed data: $\log _{10}(y)=$ $(0.85 \pm 0.07) \times \log _{10}(x)+0.70 \pm 0.09\left(\mathrm{R}^{2}=\right.$ $0.9556)$. The dashed line represents a 1:1 relationship. 


\section{Discussion}

Previous qPCR methods have targeted Trichodesmium at the genus level. In this study, I developed a qPCR assay that can distinguish the two major clades of Trichodesmium by targeting the heterocyst differentiation gene hetR. HetR has been widely studied in Trichodesmium (Janson et al., 1999; El-Shehawy et al., 2003; Lundgren et al., 2005). HetR is more variable than highly conserved regions such as $16 \mathrm{~S}$ rDNA, making it a better candidate for distinguishing between clades of Trichodesmium with qPCR. Species of Trichodesmium are $\geq 97 \%$ identical in the $16 \mathrm{~S}$ rDNA region while the inter-clade similarity for hetR was 90 - 91\% (Chapt. 2). Sequences of hetR in Trichodesmium are also more variable than nifH sequences, which were 93 - $94 \%$ similar between clades (Lundgren et al., 2001). ITS has been used as a target for qPCR in cyanobacteria (Ahlgren et al., 2006). ITS and hetR have similar inter- and intra-clade percent identities in Trichodesmium (89 - 90\% and 97 - 100\%, respectively). However, clade-specific primers designed for ITS by AlleleID were rated "poor" by the software and were incapable of producing a PCR product when tested in silico by MacVector. T. erythraeum IMS101 genome has two copies of ITS, complicating enumeration. HetR is specific to filamentous, diazotrophic cyanobacteria, reducing the chances of amplifying non-Trichodesmium DNA in the qPCR assay compared to ITS or nifH. The initial objective of this study was to target the four groups of Trichodesmium found in Janson et al. (1999) and Lundgren et al. (2005). However, in Chapt. 2, we found that the diversity of our cultured representatives spanned only two major clades, and species within each clade were too genetically similar to design a species-specific assay.

I developed an effective qPCR assay which was able to distinguish between the two clades of Trichodesmium. PCR efficiency was high, cross-amplification was low, and melting temperatures of cultured templates were reasonably close to the melting temperatures of standards. The primers for clade II occasionally formed primer dimers. To reduce the dimers, I used HPLC-purified primers and changed to a hot-start method (LightCycler 
method) to use with the liquid handler since reaction mixtures may stay at room temperatures for an extended period of time. To ameliorate the effect of the dimers on quantitation, I added a melting step to the clade II assay. Reducing the effects of primer dimers ensures that the SYBR green qPCR assay is accurate.

The relationships between cell counts and copies of hetR for cultured strains of Trichodesmium were not consistent. There were multiple copies of hetR per cell. Slopes varied from $0.86 \pm 0.10$ to $1.84 \pm 0.18$, so in several strains, copies of het $R$ cell $^{-1}$ increased as cell counts increased. There are several possible sources of the disparity between gene copy and cell counts. (1) Cyanobacteria often have multiple copies of their genome, and up to 20 copies per cell have been observed in Anabaena sp. strain PCC 7120 (Herdman et al., 1979; Cohen and Gurevitz, 2006). Trichodesmium spp. may also contain several chromosomes per cell. (2) The cells in the variety of Trichodesmium cultures sampled may have been in different cell cycle stages. While Trichodesmium cell division peaks early in the dark period, cells divide throughout the light-dark cycle (Sandh et al., 2009). (3) T. tепиe H9-4 had colonies, and counting cells within colonies using microscopy can be erroneous. (4) DNA extraction at low cell concentrations may be inefficient due to interaction of the trichomes with the filter and the beads. (5) Cell count slides were prepared using a $25 \mathrm{~mm}$ glass filter tower. The meniscus from this small tower interacted with trichomes and colonies, pulling them toward the glass. In spite of efforts to distribute cells evenly throughout the filter, cells tended to be more concentrated around the outside of the field than in the middle. Cell counts were underestimated, and this imbalance of distribution can increase with increasing cell numbers. In the future, the meniscus effect can be reduced by using a $47 \mathrm{~mm}$ filter tower. Due to variability in relationships between cells and gene copy, results from qPCR will be reported in terms of copies of hetR rather than cells.

Some Trichodesmium cultures showed evidence of PCR inhibition, so I tested a variety of DNA extraction methods to determine which method minimized inhibition. Cyanobacteria produce polysaccharides and phenolics that can inhibit PCR (Tillett and 
Neilan, 2000; Bessetti, 2007; Radström et al., 2008). The xanthogenate method was designed to extract DNA from cyanobacteria (Tillett and Neilan, 2000), but has been shown to inhibit qPCR (Foster et al., 2007). The MoBio soil method, which specializes in removing compounds that inhibit PCR, also resulted in low amplification. Low amplification may be due to a combination of DNA extraction efficiency and PCR inhibition. The MoBio plant method gave the best results in our qPCR assay when compared to all the other methods tried and will be used in future field studies.

Acknowledgements. I would like to thank R. Gast and her lab for the use of the Mini-Beadbeater 8, H. Sosik for her advice in image-processing, P. Kovesi for making his MATLAB image-processing scripts available, and the Keck facility at the Marine Biological Laboratory and Agencourt Bioscience Corporation for sequence data. I would like to thank J. Waterbury and S. Doney for their advice in interpreting the data for this chapter, E. Webb and E. DeLong for their advice on designing qPCR primers and standards, T. Mincer for his help in troubleshooting the qPCR methods, and E. Zinser and Z. Johnson for their advice on extracting DNA from large filters. Mahalo to the scientists who shared their expertise in dealing with DNA extractions, PCR inhibition, and the disparity between gene copy and cell counts: M. Church, E. DeLong, R. Foster, T. Mincer, M. Rappé, G. Steward, and J. Zehr. I am grateful to the Chisholm and DeLong labs at MIT for the use of their LightCycler-480 and liquid handler, especially to R. Malmstrom who graciously trained me to use the machines. I would like to express my gratitude to P. D. Chappell for her comments on drafts of this manuscript. This research was supported by the Woods Hole Oceanographic Institution (WHOI) Ocean Life Institute (OLI) grant to J. Waterbury and the Center for Microbial Oceanography Research and Education (CMORE), an NSF Science and Technology Center (EF-0424599). 


\title{
CHAPTER 4
}

\section{The Distribution of Trichodesmium field populations}

\begin{abstract}
A real-time quantitative polymerase chain reaction (qPCR) assay targeting two different clades of Trichodesmium was applied to depth profiles in the Atlantic and Pacific Oceans. Clade II (Trichodesmium thiebautii, Trichodesmium tenue, Trichodesmium hildebrandtii, and Trichodesmium pelagicum) was typically more numerous in the open ocean than clade I (Trichodesmium erythraeum and Trichodesmium contortum). Concentrations of both clades were higher in the Atlantic than in the Pacific during the timeframe of this study. Neither clade correlated with surface phosphorus $(\mathrm{P})$ or iron $(\mathrm{Fe})$ concentrations, but clade I had deeper and colder distributions than clade II. Some sequences of qPCR products matched varieties of Trichodesmium previously found in the field but not in culture. Numerical models parameterizing $\mathrm{N}_{2}$ fixation are often based on clade I, while clade II was more numerically dominant in this study. Model results and our understanding of Trichodesmium and global $\mathrm{N}_{2}$ fixation may be distorted if there are important ecological differences between clades I and II.
\end{abstract}




\section{Introduction}

Cyanobacteria of the genus Trichodesmium are responsible for a large fraction of the $\mathrm{N}_{2}$ fixation in oligotrophic oceans (Capone et al., 1997). There are two major clades of Trichodesmium: clade I comprising of Trichodesmium erythraeum and Trichodesmium contortum, and clade II comprising of Trichodesmium thiebautii, Trichodesmium tenue, Trichodesmium hildebrandtii, and Trichodesmium pelagicum (Chapt. 2).

The distribution of Trichodesmium spp. can be limited by a variety of physical, chemical, and biological factors. Trichodesmium thrives in stratified, warm water with high light (Capone et al., 1997; LaRoche and Breitbarth, 2005). Nutrients such as phosphorus (P) and iron (Fe) affect Trichodesmium growth and $\mathrm{N}_{2}$ fixation rates (Wu et al., 2000; Moore et al., 2004). Factors affecting the mortality of Trichodesmium are not as well understood. Grazing on Trichodesmium is considered to be low (Carpenter et al., 1987), and mortality due to viral lysis may be important but is not well-constrained (Hewson et al., 2004). Evidence for apoptosis has been found in both culture and field populations of Trichodesmium in response to nutrient stress and high light (Berman-Frank et al., $2004,2007)$, but the function of this method of mortality and its extent in field populations are not well-understood.

Trichodesmium spp. often form extensive "blooms" that are visible from space (Capone et al., 1997). These regions of high density occur in warm water $\left(>26^{\circ} \mathrm{C}\right)$ with low windspeed (Carpenter and Capone, 1992). Rather than being a product of high growth and low mortality, these surface slicks of Trichodesmium arise from the accumulation of biomass due to periods of low mixing followed by Langmuir circulation. Trichodesmium cells are viable during the early stages of the slick (Carpenter and Capone, 1992), but become inactive, possibly due to photooxidative stress and autolysis (Berman-Frank et al., 2004).

Field populations of Trichodesmium have previously been enumerated using cell counts (Letelier and Karl, 1996; Post et al., 2002; Tyrrell et al., 2003), the video plankton 
recorder (VPR) (Davis and McGillicuddy, 2006), and real-time quantitative polymerase chain reaction (qPCR) targeting the nitrogenase enzyme gene nifH (Church et al., 2005; Foster et al., 2007; Goebel et al., 2007). In a depth profile measured with qPCR at Sta. ALOHA, Church et al. (2005) found that Trichodesmium spp. abundance was high at the surface with a subsurface peak near the mixed layer depth (MLD). During a 2-year study at Sta. ALOHA coupling qPCR and modeling, Goebel et al. (2007) found that Trichodesmium spp. dominated $\mathrm{N}_{2}$ fixation in the summer and autumn, and that abundance was highly variable. Foster et al. (2007) found that Trichodesmium spp. abundances in the western tropical Atlantic Ocean were high in regions of low surface nutrients, high salinity, and high temperatures. All of these previous qPCR studies have targeted the genus Trichodesmium as a whole using nifH. I developed a method to distinguish the two major clades of Trichodesmium by targeting the gene for the heterocystous differentiation protein hetR (Chapt. 3).

In this study, I used the qPCR assay developed in Chapt. 3 to enumerate the two clades of Trichodesmium in field samples collected from depth profiles in the west Pacific warm pool from Hawai'i to Australia (Fig. 4-1A), the Equatorial Atlantic Ocean from Cape Verde to Barbados (Fig. 4-1B), and the South Pacific Ocean between Australia and New Caledonia (Fig. 4-1C). I found that clade II dominates open ocean populations and that the two clades have different depth and temperature distributions.

\section{Methods}

Sample collection, storage, and extraction. Field samples were collected during an east-west transect of the Equatorial Atlantic Ocean in July, 2006 on the R/V SewardJohnson (SJ0609), on a north-south transect of the western Pacific Ocean in JanuaryFebruary, 2007 as part of the West Pacific Warm Pool cruise (WP ${ }^{2}$, KM0701) on the R/V Kilo Moana, and in the South Pacific Ocean east of Australia in March, 2007 on the R/V

Kilo Moana (KM0703). Sea water was collected at specific depths from 12 L Niskin bottles mounted on a rosette equipped with a conductivity, temperature, and depth sensor 

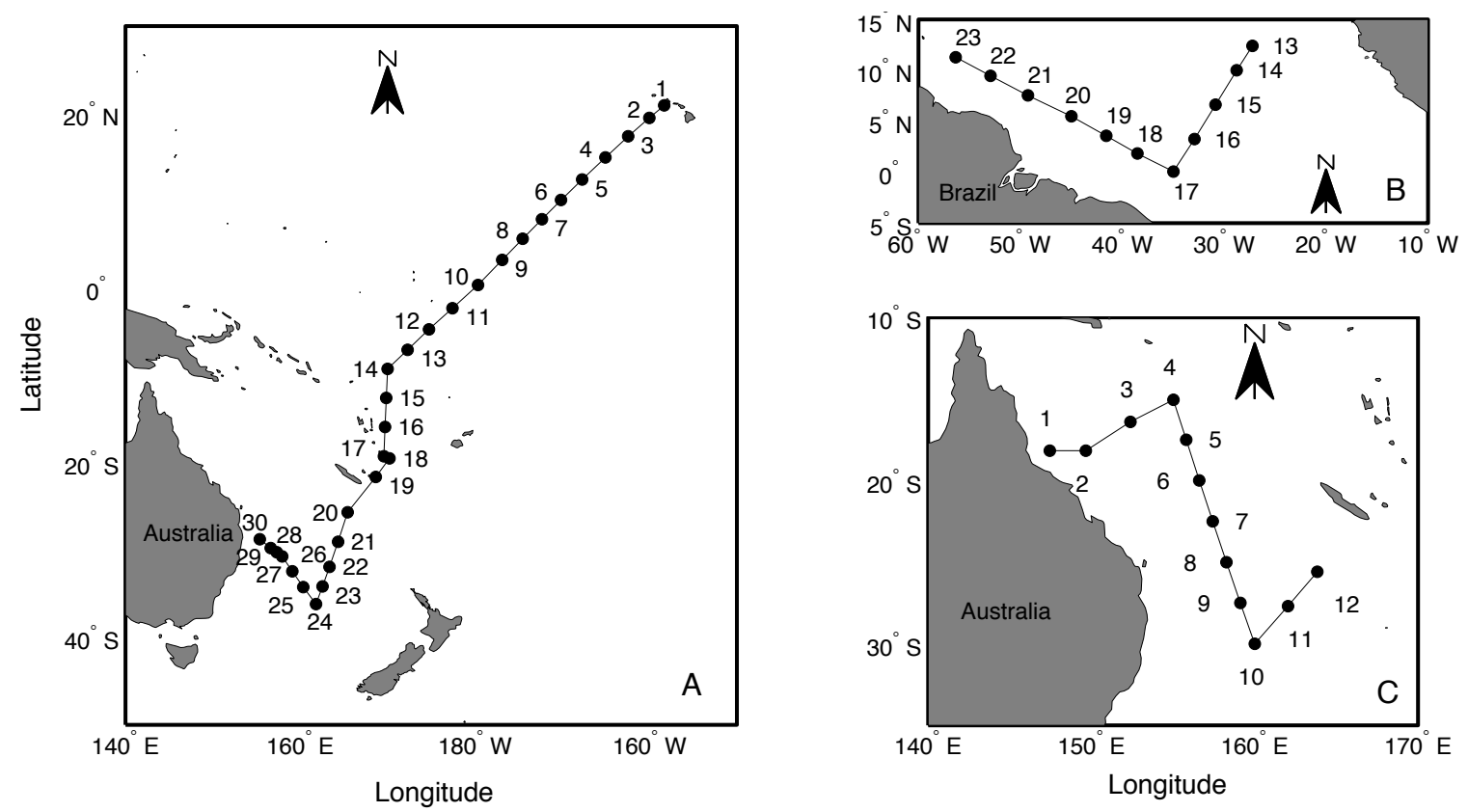

Figure 4-1: Map of cruise tracks for (A) west Pacific warm pool cruise KM0701 (January - February, 2007), (B) Equatorial Atlantic cruise SJ0609 (July, 2006), and (C) South Pacific cruise KM0703 (March, 2007).

(CTD). Mixed layer depth (MLD) for each profile was determined using potential density. Samples from four depths down to the deep chlorophyll maximum (DCM) were collected for SJ0609 and KM0703. Six depths down to $150 \mathrm{~m}$ and including the DCM were collected from profiles of KM0701. During a Trichodesmium bloom between Stas. KM0701-16 and 17, a sample was taken from $2 \mathrm{~m}$ using a hand-deployed Niskin bottle. At the center of the warm pool (Sta. KM0701-14), depth profiles were taken every $4 \mathrm{~h}$ for $24 \mathrm{~h}$ during an Eulerian diel station. Eight to twelve L of sea water was gravity-filtered through a $47 \mathrm{~mm}, 5 \mu \mathrm{m}$ polycarbonate filter (Millipore). The filters were contained in polypropylene in-line filter holders (Advantec MFS) and silicone tubing that were washed with $10 \%$ bleach and rinsed with distilled water between each deployment. The filtrate was measured and the filters were placed in $1.8 \mathrm{~mL}$ cryo tubes (Nunc) and frozen in liquid $\mathrm{N}_{2}$. Samples were homogenized by beadbeating and DNA was extracted with the PowerPlant DNA Isolation Kit (MoBio) using the methods described in Chapt. 3. In addition to 
the extraction, $50 \mu \mathrm{L}$ of eluate was used in the optional clean-up protocol following manufacturer's instructions (MoBio), and the final pellet was resuspended in $50 \mu \mathrm{L}$ of buffer PB6 and $50 \mu \mathrm{L}$ of molecular-grade water (5Prime). West Pacific warm pool samples from Stas. KM0701-2 - 14 were first extracted using the DNeasy 96 Plant Kit (Qiagen) as described in Chapt. 3, and then stored lysates were extracted using the MoBio plant method. All extractions and bead tubes containing filters and lysates were stored at $-20^{\circ} \mathrm{C}$.

During the Atlantic cruise, $10 \mathrm{~L}$ were gravity-filtered on $47 \mathrm{~mm}, 5 \mu \mathrm{m}$ polycarbonate filters (Millipore), fixed with formalin, mounted on slides, and stored at $-20^{\circ} \mathrm{C}$ for cell counts. Slides were generously provided by the Zehr laboratory (University of CaliforniaSanta Cruz). Slides were viewed using epifluorescence (CY3LP filter) with a Zeiss Axioplan 2 microscope under a $10 \times$ Plan-Neoflaur objective lens. Eighty micrographs per slide were taken over the full area of the filter using a Zeiss Axiocam HRC digital camera and Axiovision 4.6.3 software. Cells were counted as described in Chapt. 3 either manually using ImageJ software (http://rsbweb.nih.gov/ij) or using the automated method if there was little epifluorescence from other cyanobacteria.

Nutrient analyses from SJ0709 and KM0701 were sent to the College of Oceanic and Atmospheric Sciences, Oregon State University, and dissolved inorganic phosphorus (DIP) was analyzed using a Technicon AutoAnalyzer II by J. Jennings with a detection level of $6 \mathrm{nmol} \mathrm{L}{ }^{-1}$. KM0703 nutrients were analyzed by R. Horak at Georgia Tech using an AutoAnalyzer. Dissolved nutrient concentrations from SJ0609 were provided by P. D. Chappell (Chappell, 2009), from KM0701 were provided by E. Webb (Hynes et al., 2009), and from KM0703 were provided by R. Horak. Dissolved Fe concentrations for SJ0609 and KM0701 were provided by P. D. Chappell (Chappell, 2009).

Quantitative polymerase chain reaction (qPCR). Reactions were performed in triplicate as described in Chapt. 3 in a LightCycler-480 (Roche) with $20 \mu \mathrm{L}$ reactions in white 384-well plates sealed with optically clear sealing foil and the LightCycler SYBR Green Master (Roche). Primers targeting hetR were clade-specific and standard curves were de- 
rived from cloned standards (Chapt. 3). PCR efficiency of each sample was determined as described by Short et al. (2004) and Foster et al. (2009), using a fourth replicate of samples spiked with 0.07 pg of cloned standard. Samples with efficiency $<95 \%$ were considered to be inhibited and the compensated concentration was calculated. Selected qPCR products were purified using the QIAquick PCR Purification Kit (Qiagen) and sent to Northwoods DNA, Inc. for sequencing. Linear correlations between qPCR results and ancillary data were tested for significance using a t-test on the correlation coefficient $\mathrm{R}(\alpha$ $=0.05)$.

\section{Results}

Transect hydrography, nutrients, and qPCR. Open ocean Trichodesmium populations were higher in the Atlantic than in the Pacific Ocean and generally dominated by clade II (Figs. 4-2 -4-4). Stations with populations made up almost exclusively of clade II were Stas. SJ0609-18 (Fig. 4-2A, B) and KM0703-6 and 12 (Fig. 4-4A, B). Notable exceptions to clade II dominance were Stas. SJ0609-14 (Fig. 4-2A, B), KM0701-5 - 12 and $21-23$ (Fig. 4-3A, B), and the bloom station during KM0701, where concentrations of clade I were higher than clade II. Cell counts for the Atlantic stations resulted in a linear fit of $\log _{10}$ (total copies hetR $\left.\mathrm{L}^{-1}\right)$ to $\log _{10}\left(\right.$ total cells $\left.\mathrm{L}^{-1}\right)$ of $\log _{10}(y)=(0.86 \pm 0.28) \times$ $\log _{10}(x)+1.66 \pm 0.14\left(\mathrm{R}^{2}=0.4104\right)($ Fig. 4-5)

Maximum transect concentrations of Trichodesmium for clade I and clade II in the Equatorial Atlantic were $8.1 \times 10^{4}$ and $1.5 \times 10^{6}$ copies hetR $\mathrm{L}^{-1}$, respectively (Fig. 4-2A, B). Ratios of clade II:clade I non-zero concentrations ranged from 0.007 - 427.9 with only one ratio $<1$. The MLD of the Equatorial Atlantic cruise varied from 17 - 85 $m$ with shallower depths at the east and west ends of the transect (Fig. 4-2C-E). Surface temperature ranged from $26.8-29.1{ }^{\circ} \mathrm{C}$ (Fig. 4-2C), and surface salinity ranged from 13.2 - 36.2 PSU with a lens of fresher surface water at the west end of the transect (Fig. 4-2D). Surface DIP concentrations ranged from 0.014 - $0.080 \mu \mathrm{mol} \mathrm{L}^{-1}$ (Fig. 4-2E), and surface dissolved Fe concentrations ranged from 0.09 - 1.89 nmol L ${ }^{-1}$ (Chappell, 2009). 
During the west Pacific warm pool cruise, maximum transect concentrations for clade I and II were $4.0 \times 10^{4}$ and $6.4 \times 10^{5}$ copies $h e t R \mathrm{~L}^{-1}$, respectively (Fig. 4-3A, B). At a surface slick of Trichodesmium between Stas. KM0701-16 and 17, surface concentrations were $8.0 \times 10^{6}$ copies het $R \mathrm{~L}^{-1}$ for clade I and $1.9 \times 10^{6}$ copies het $R \mathrm{~L}^{-1}$ for clade II. Ratios of non-zero clade II:clade I concentrations ranged from 0.06 to 392.2, and a majority of the ratios were $>1$. The MLD ranged from $11-118 \mathrm{~m}$, becoming more shallow as the transect moved south (Fig. 4-3C, D). Surface temperature ranged from 20.8 $30.8^{\circ} \mathrm{C}$ with the warmest temperature at Sta. KM0701-14 and the coolest temperature at Sta. KM0701-24, the southernmost station and turning point (Fig. 4-3C). Surface salinity ranged from 33.9 - 35.9 PSU with regions of high salinity near the Equator and south of New Caledonia (Fig. 4-3D). Surface DIP concentrations ranged from 0.050 - $0.324 \mu \mathrm{mol}$ $\mathrm{L}^{-1}$ with maximum [DIP] at the Equator (Fig. 4-3E) (Hynes et al., 2009), and surface dissolved Fe concentrations ranged from 0.09 - $1.40 \mathrm{nmol} \mathrm{L}^{-1}$ (Chappell, 2009).

Maximum Trichodesmium concentrations for the South Pacific cruise were $3.1 \times 10^{3}$ and $5.2 \times 10^{4}$ copies het $R \mathrm{~L}^{-1}$ for clade I and clade II, respectively (Fig. 4-4A, B). Ratios of clade II:clade I where concentrations of both clades were non-zero ranged from 0.2 125.6. For the majority of samples, clade II:clade I was $>1$. MLD spanned 21 - $61 \mathrm{~m}$, the deepest mixed layers occurring at the southeastern end of the transect (Fig. 4-4C-E). Surface temperature ranged from $24.2-29.2^{\circ} \mathrm{C}$ (Fig. 4-2C), and surface salinity ranged from 34.4 - 35.9 PSU (Fig. 4-2D). There was a region of relatively cool, salty water off the east coast of Australia at the northern end of the transect. Surface DIP concentrations ranged from below detection level (BDL) - $0.554 \mu \mathrm{mol} \mathrm{L}^{-1}$ (Fig. 4-2E) (R. Horak et al, unpublished data).

Across all three cruises, there was a low but significant correlation between the concentrations of clade I and clade II (Fig. 4-6). A linear fit through the $\log _{10}-\log _{10}$ transformed non-zero data of clade II (copies het $R \mathrm{~L}^{-1}$ ) versus clade I (copies het $\mathrm{L}^{-1}$ ) of the combined data had the form $\log _{10}(y)=0.75 \times \log _{10}(x)+1.70\left(\mathrm{R}^{2}=0.27, p=\right.$ $1.3 \times 10^{-7}$ ). This relationship was not consistent for each cruise. The Equatorial Atlantic 


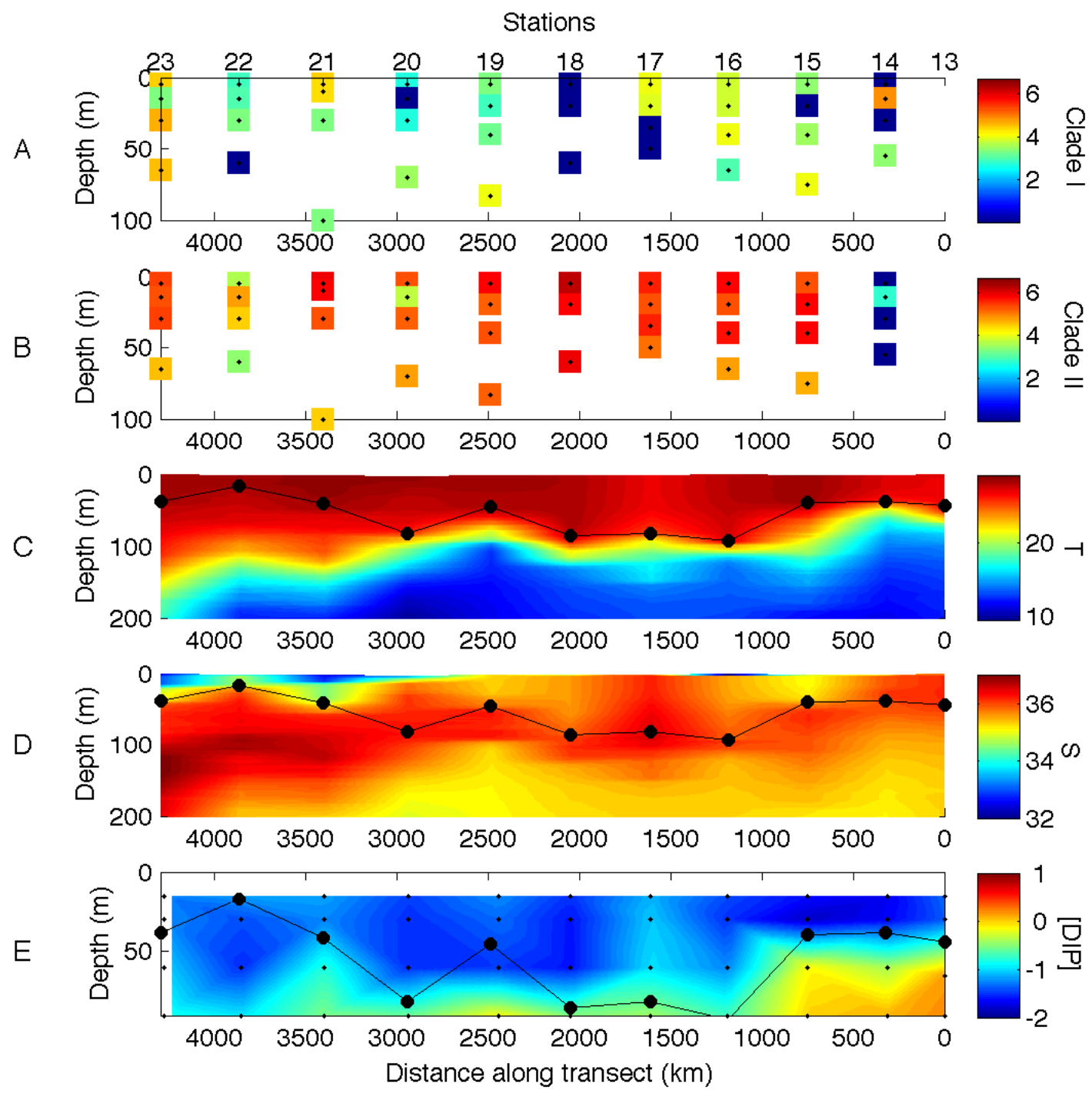

Figure 4-2: Data from the Equatorial Atlantic cruise SJ0609. The X-axes represent distance along the transect in $\mathrm{km}$ and have been reversed to show the data in a west-east orientation. Station numbers are indicated at the top. The y-axes represent depth (m). Black circles connected by a black line indicate mixed layer depth (MLD). (A) Plot of qPCR data for Trichodesmium clade I. Colored squares represent $\log _{10}$ (copies hetR $\mathrm{mL}^{-1}$ ). Black dots indicate the location of the qPCR samples. (B) Plot as described in (A) for Trichodesmium clade II. (C) Potential temperature in ${ }^{\circ}$ C. (D) Salinity in PSU. (E) $\log _{10}\left([\mathrm{DIP}]\right.$ in $\left.\mu \mathrm{mol} \mathrm{L}{ }^{-1}\right)$. Black dots indicate location of samples taken. 


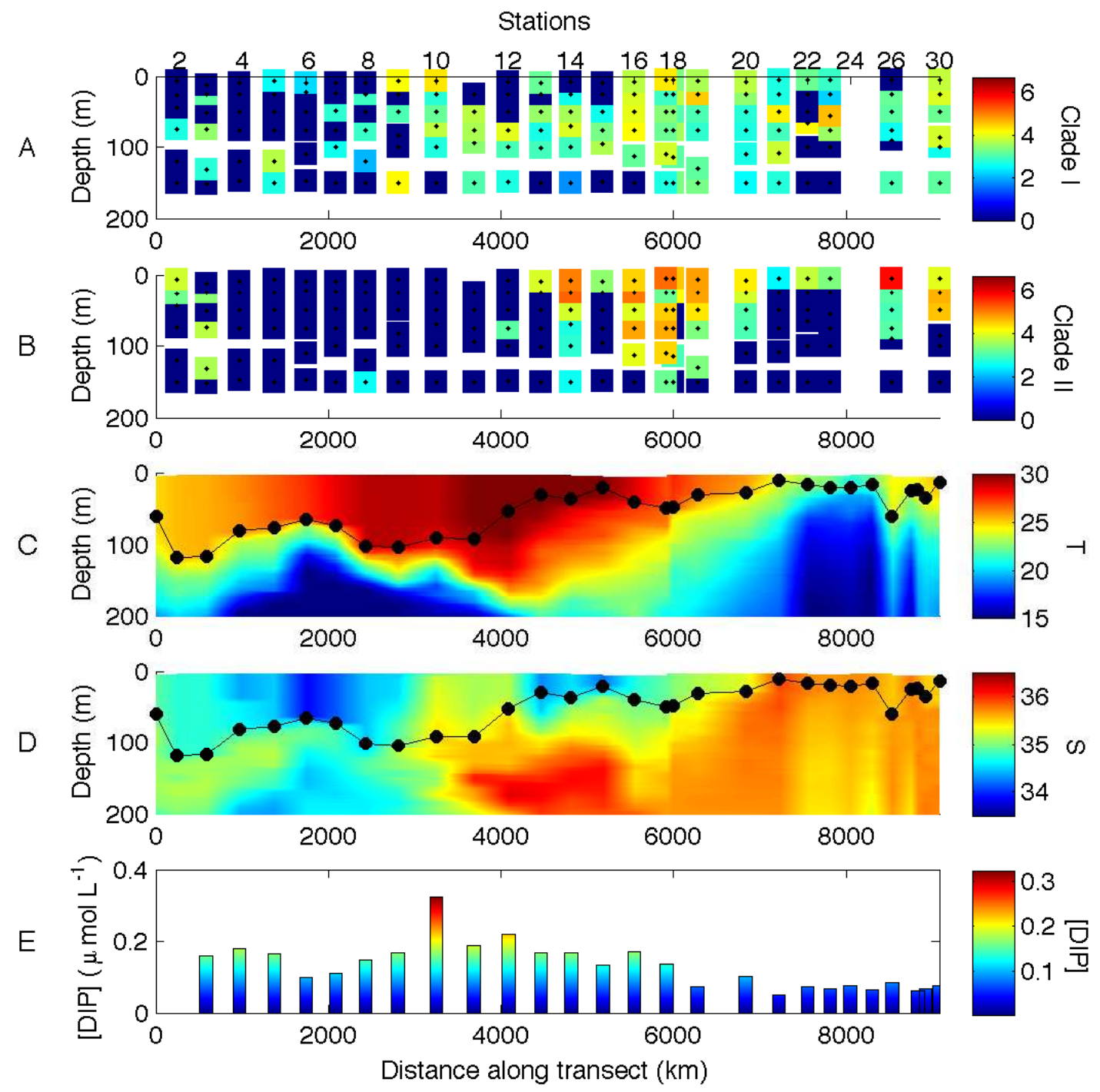

Figure 4-3: Data from the west Pacific warm pool cruise KM0701. The x-axes represent distance along the transect in $\mathrm{km}$. Station numbers are indicated at the top. The y-axes in A - D represent depth $(\mathrm{m})$. Black circles connected by a black line indicate mixed layer depth (MLD). (A) Plot of qPCR data for Trichodesmium clade I. Colored squares represent $\log _{10}$ (copies het $\mathrm{mL}^{-1}$ ). Black dots indicate the location of the qPCR samples. (B) Plot as described in (A) for Trichodesmium clade II. (C) Potential temperature in ${ }^{\circ} \mathrm{C}$. (D)

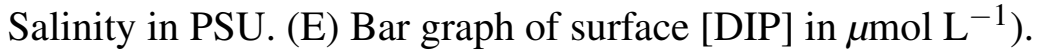



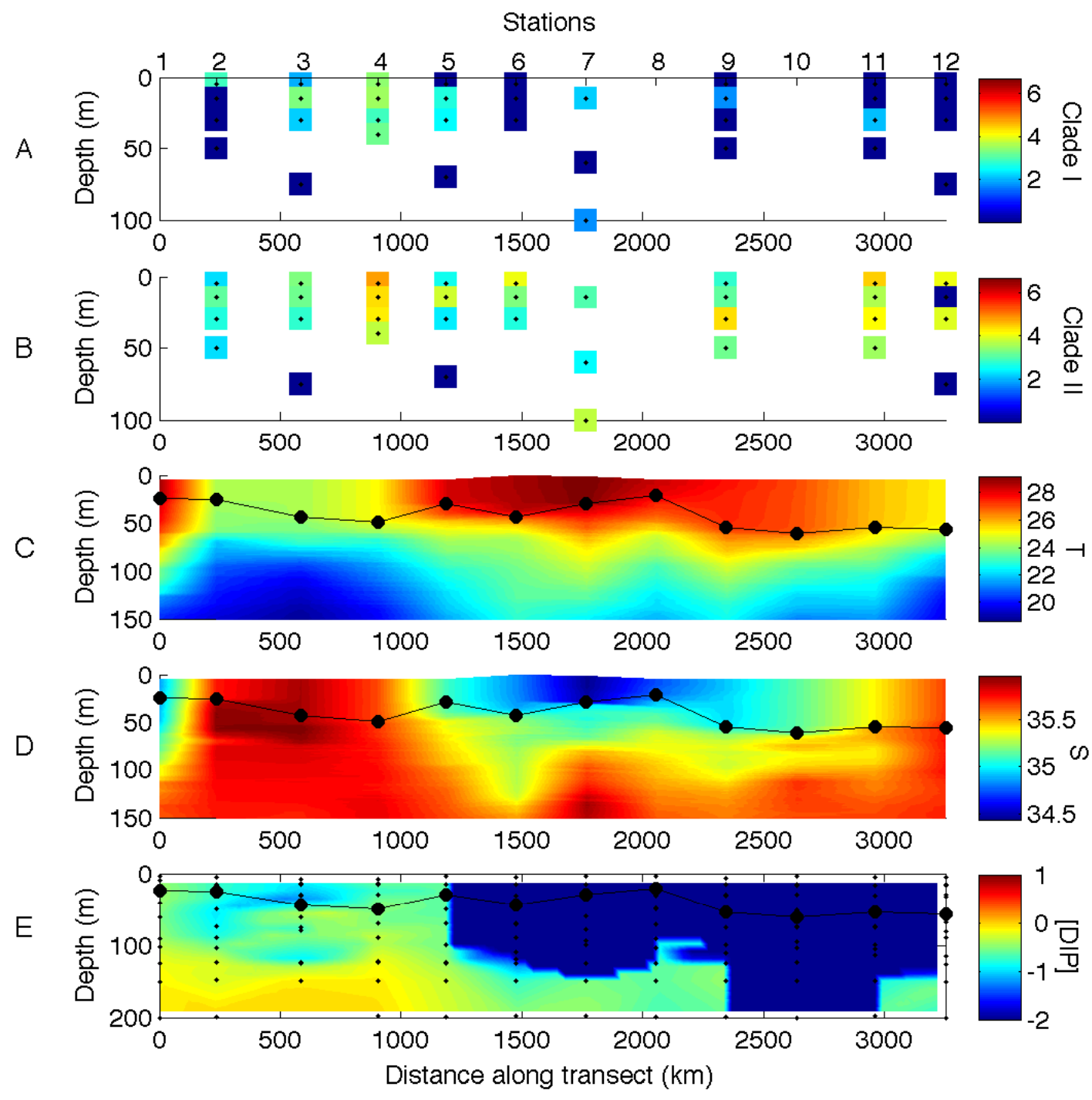

Figure 4-4: Data from the South Pacific cruise KM0703. The x-axes represent distance along the transect in $\mathrm{km}$ and station numbers are indicated at the top. The y-axes represent depth $(\mathrm{m})$. Black circles connected by a black line indicate mixed layer depth (MLD). (A) Plot of qPCR data for Trichodesmium clade I. Colored squares represent $\log _{10}$ (copies het $R \mathrm{~mL}^{-1}$ ). Black dots indicate the location of the qPCR samples. (B) as described in (A) for Trichodesmium clade II. (C) Potential temperature in ${ }^{\circ}$ C. (D) Salinity in PSU. (E) $\log _{10}\left([\mathrm{DIP}] \mu \mathrm{mol} \mathrm{L}{ }^{-1}\right)$. Black dots indicate location of samples taken. 

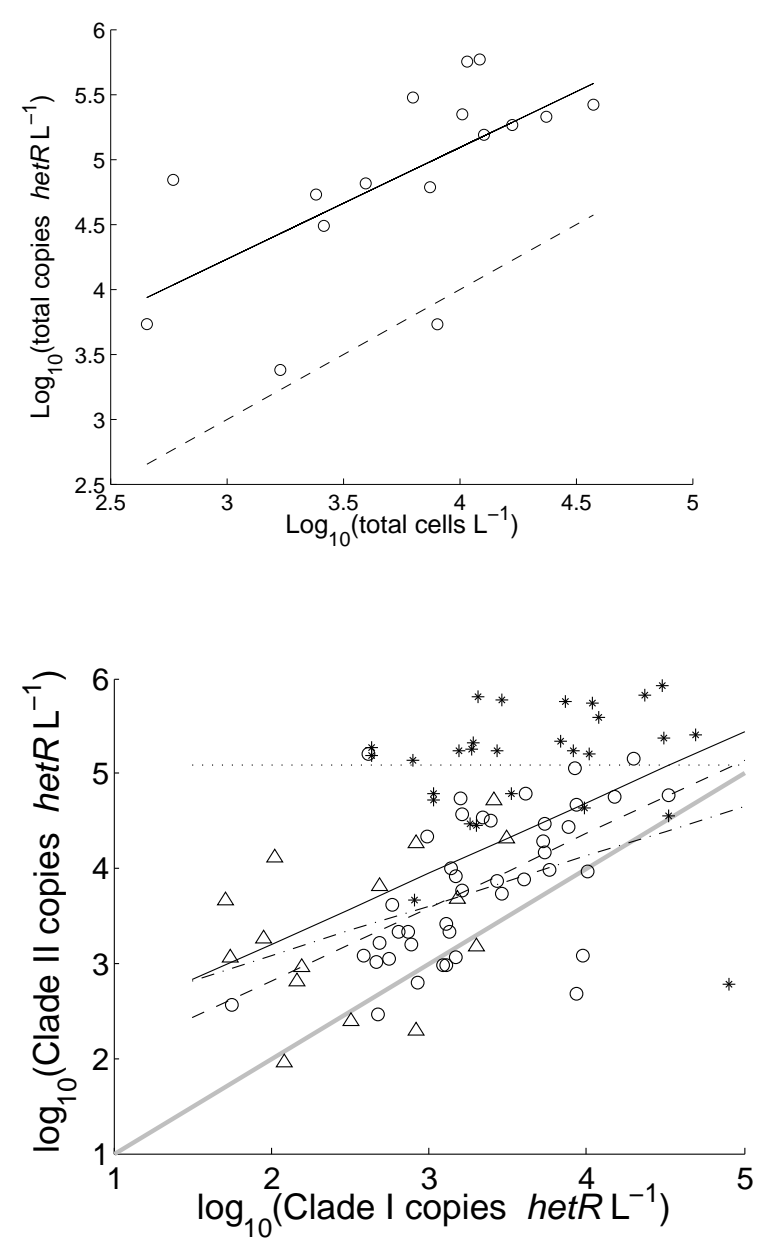

Figure 4-5: Total qPCR results versus cell counts of SJ0609. The y-axis represents $\log _{10}$ (total copies of hetR $\mathrm{L}^{-1}$ ) and the $\mathrm{x}$ axis represents $\log _{10}\left(\right.$ total cells $\left.\mathrm{L}^{-1}\right)$. Linear fit of the $\log _{10}$-transformed data is represented by the solid line. The dashed line represents a 1:1 relationship.

Figure 4-6: Comparison of concentrations of the two Trichodesmium clades. The $\mathrm{x}$-axis represents $\log _{10}$ (copies het $R \mathrm{~L}^{-1}$ ) for clade I. The $y$-axis represents $\log _{10}$ (copies het $R$ $\mathrm{L}^{-1}$ ) for clade II. The grey line indicates a one-to-one relationship. Black lines represent linear fits of the $\log _{10}-\log _{10}$ transformed data. The solid line was the fit through the points for all three cruises. The dotted line was fit through SJ0609 (asterisks), the dashed line was fit through KM0701 (circles), and the dash-dot line was fit through KM0703 (triangles).

cruise did not have a significant relationship between the concentrations of the two clades: $\log _{10}(y)=0.004 \times \log _{10}(x)+5.07\left(\mathrm{R}^{2}=1.49 \times 10^{-5}, p=0.492\right)$. The correlation between the two clades was significant in the west Pacific warm pool: $\log _{10}(y)=0.77 \times$ $\log _{10}(x)+1.27\left(\mathrm{R}^{2}=0.32, p=4.2 \times 10^{-5}\right)$, but the fit through the South Pacific cruise data was not significant at a $95 \%$ confidence level: $\log _{10}(y)=0.52 \times \log _{10}(x)+2.03$ $\left(\mathrm{R}^{2}=0.16, p=0.07\right)$. 

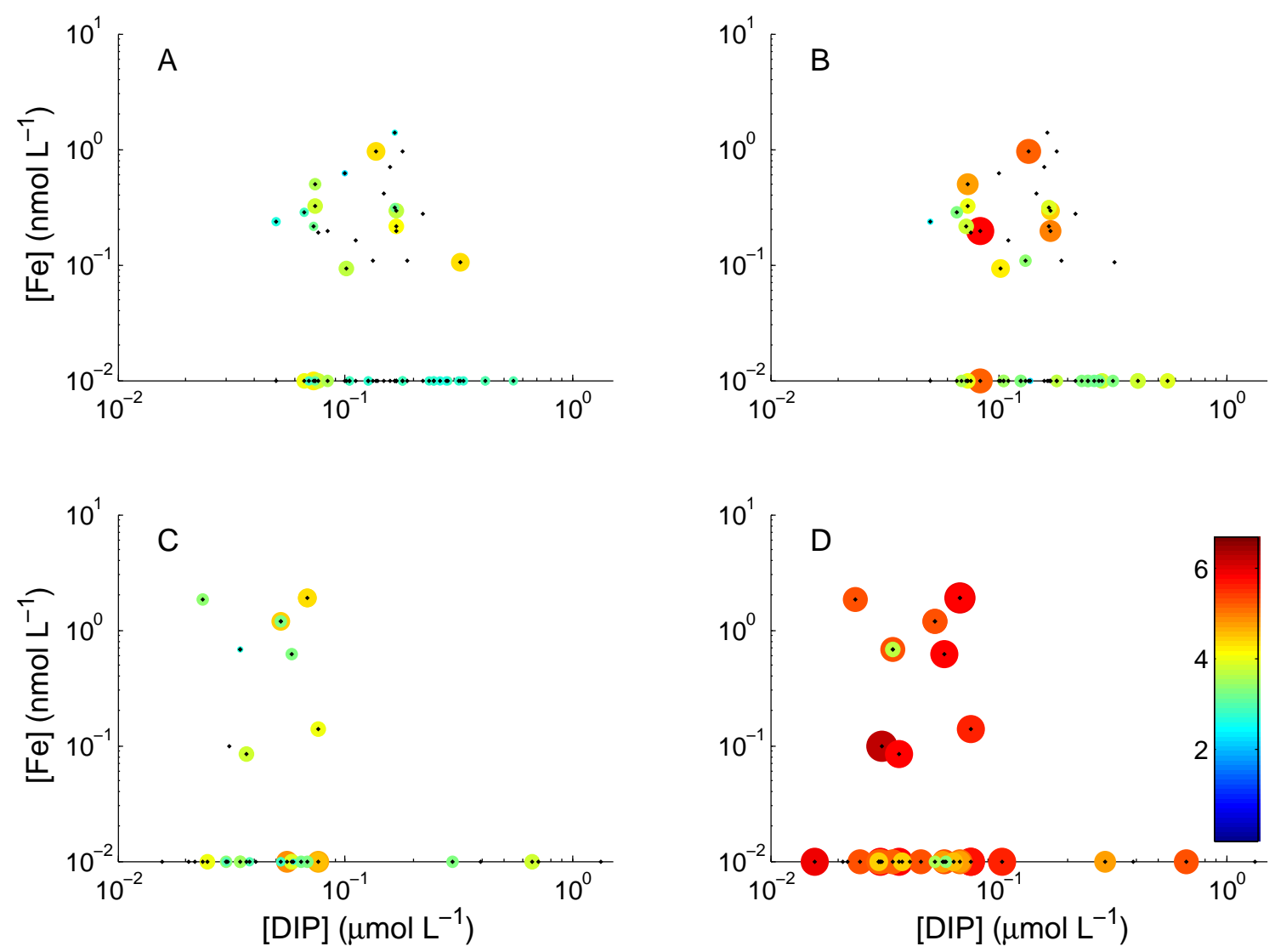

Figure 4-7: Plot of Fe (nmol L $\left.{ }^{-1}\right)$ versus DIP $\left(\mu \mathrm{mol} \mathrm{L}^{-1}\right)$ represented by black dots. Circle color and size represent Trichodesmium concentrations $\left(\log _{10}\left(\operatorname{copies~hetR~} \mathrm{L}^{-1}\right)\right)$. Colored circles along the $\mathrm{X}$-axis represent samples for which $\mathrm{Fe}$ concentrations were not available. (A) Clade I, Pacific samples; (B) clade II, Pacific samples; (C) clade I, Atlantic samples; (D) clade II, Atlantic samples.

Nutrients and Trichodesmium. There were no distinct correlations between concentrations of either clade of Trichodesmium and DIP or Fe (Fig. 4-7). Each ocean basin had similar ranges of Fe, but the Pacific samples (Fig. 4-7A-B) had higher concentrations of DIP than the Atlantic (Fig. 4-7C-D). Ratios of clade II:clade I were lower and more variable in the Pacific (Fig. 4-8A) than in the Atlantic (Fig. 4-8B). There was no correlation between Trichodesmium clade ratios and Fe and high clade II:clade I ratios appear to be associated with low DIP (Fig. 4-8A-B). 

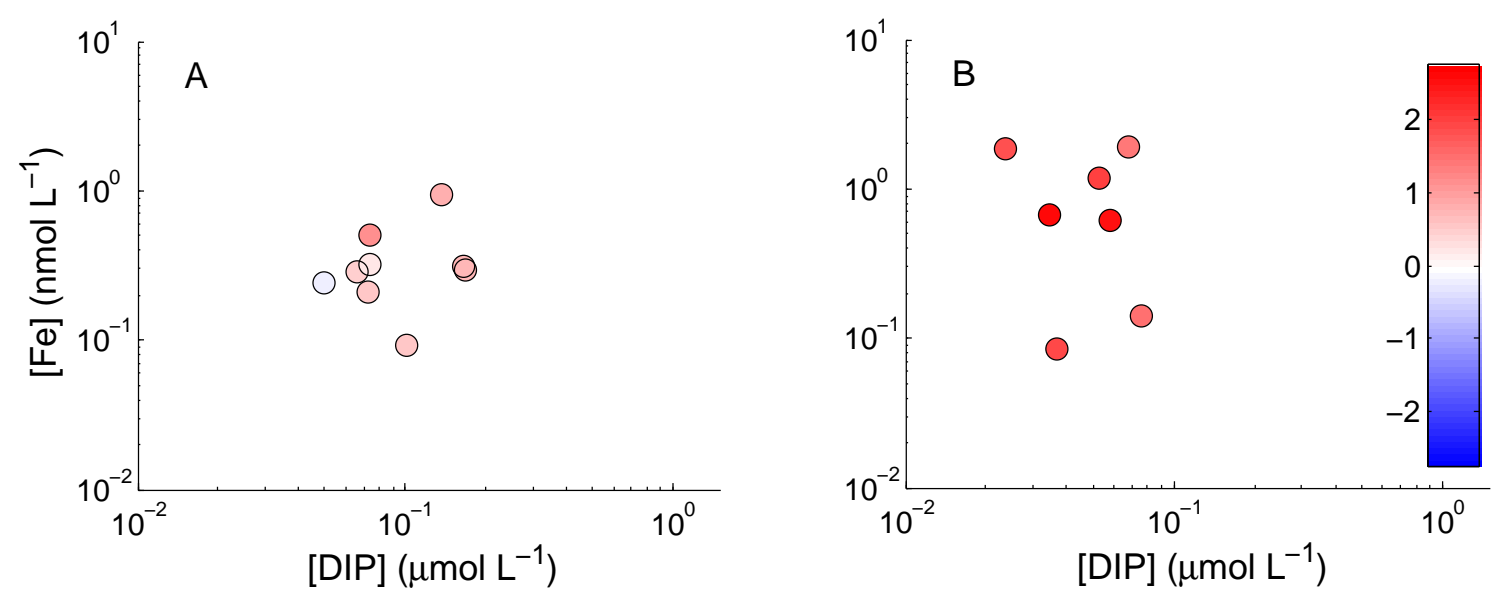

Figure 4-8: Ratio of clade II:clade I concentrations in nutrient space. Y-axis represents Fe concentration (nmol L $\left.{ }^{-1}\right)$ and $\mathrm{x}$-axis represents DIP concentration $\left(\mu \mathrm{mol} \mathrm{L}{ }^{-1}\right)$. Circle color represents $\log _{10}$-transformed ratios of clade II concentrations (copies het $R \mathrm{~L}^{-1}$ ) to clade I concentrations (copies hetR $\mathrm{L}^{-1}$ ). (A) Pacific samples, (B) Atlantic samples.

Temperature, depth, and Trichodesmium. Clade I had broader temperature and depth distributions than clade II from all three cruises (Fig. 4-9-4-10). Neither clade amplified in the few samples collected at temperatures $<16^{\circ} \mathrm{C}$ (Fig. 4-9A-B, Fig. 4-10A, C, E). Clade I amplified in colder and deeper areas in the Pacific than clade II (Fig. 4-9A-B). A vast majority of the Atlantic samples were taken from the mixed layer, so the ranges of temperature and depth were limited (Fig. 4-9C-D). High concentrations of clade I were found throughout the sample space for both ocean basins (Fig. 4-9A, C), while concentrations of clade II were higher in shallow, warm water (Fig. 4-9B, D). For the samples from $16-18^{\circ} \mathrm{C}, 18-20^{\circ} \mathrm{C}$, and $20-22^{\circ} \mathrm{C}$ the proportions of clade I samples amplifying were $0.33,0.80$, and 0.85 , respectively (Fig. 4-10C). In comparison, the proportions of clade II samples amplifying at the above temperatures were $0.17,0$, and 0.15 , respectively (Fig. 4-10E). As depth increased, the proportion of clade I samples that amplified remained above 0.5 while the proportion of clade II samples that amplified decreased below 0.5 when depth $\geq 30 \mathrm{~m}$ (Fig. 4-10D, F). Ratios of clade II: clade I concentrations were lower in the Pacific than the Atlantic (Fig. 4-11A-B). In both basins, clade II:clade I ratios were low at temperatures less than $25^{\circ} \mathrm{C}$ and depths greater than $50 \mathrm{~m}$ (Fig. 4-11A-B). 

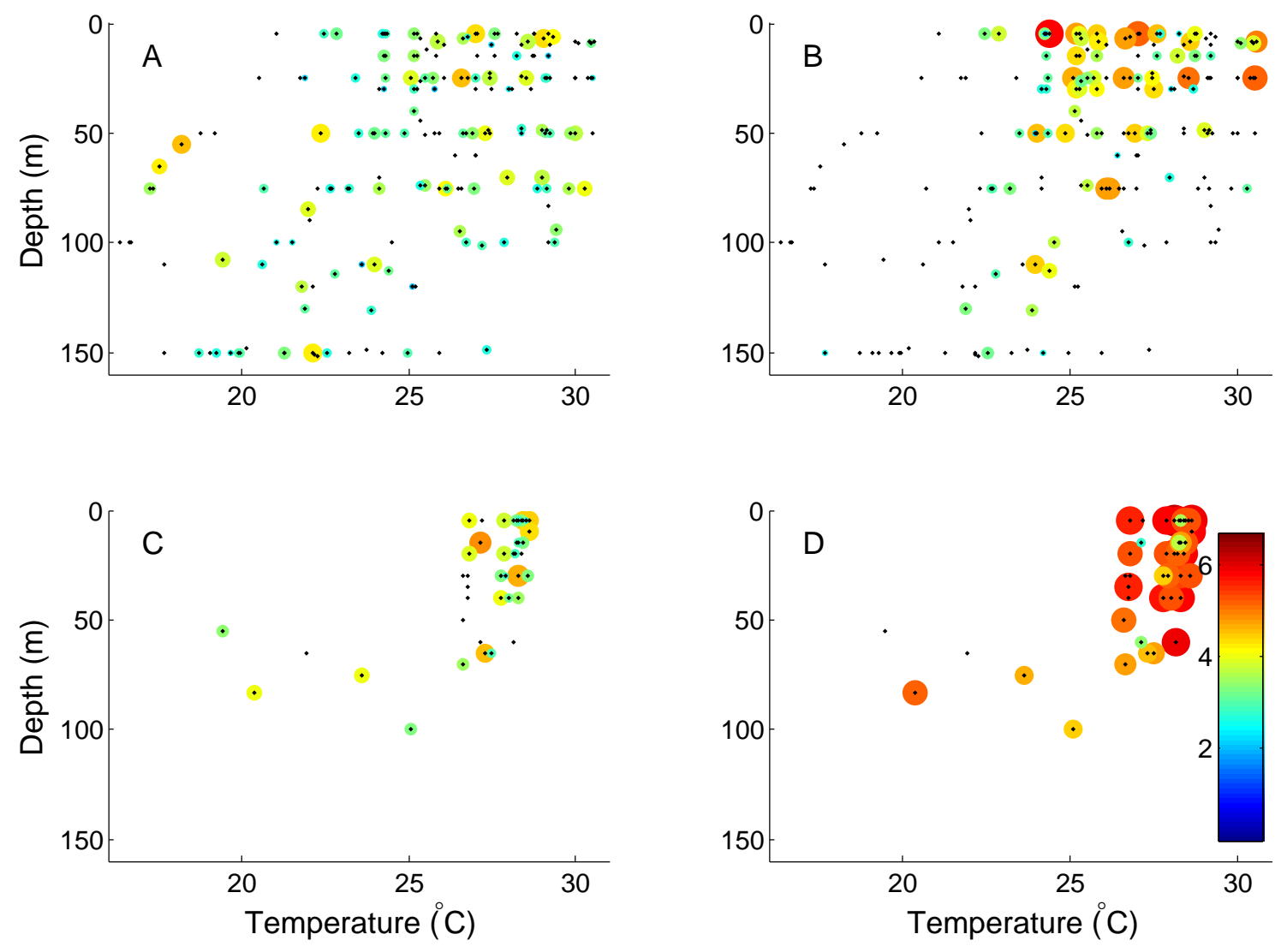

Figure 4-9: Plot of depth $(\mathrm{m})$ versus temperature $\left({ }^{\circ} \mathrm{C}\right)$ represented by black dots. Circle color and size represent Trichodesmium concentrations ( $\left.\log _{10}\left(\operatorname{copies} h e t R \mathrm{~L}^{-1}\right)\right)$. (A) Clade I, Pacific samples; (B) clade II, Pacific samples; (C) clade I, Atlantic samples; (D) clade II, Atlantic samples. 

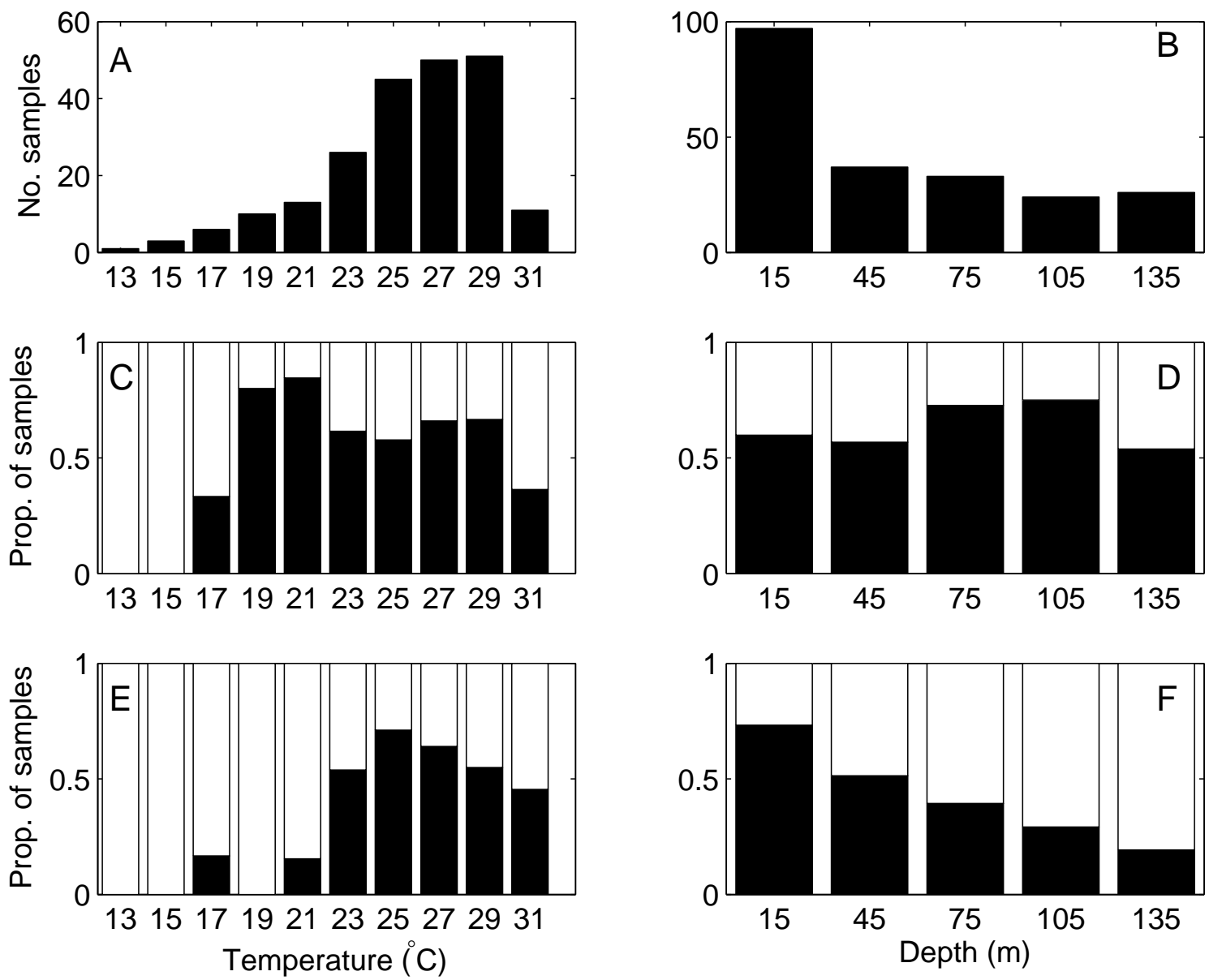

Figure 4-10: Histograms of temperatures (A, C, E) and depths (B, D, F) of qPCR samples from all three cruises. (A) Histogram of potential temperature $\left({ }^{\circ} \mathrm{C}\right)$ for all qPCR samples. (B) Histogram of depths (m) for all qPCR samples. (C) Clade I stacked bar graph of proportions of samples at each temperature for positive qPCR results (black) and negative qPCR results (white). (D) Clade I stacked bar graph of proportions of samples at each depth for positive qPCR results (black) and negative qPCR results (white). (E) Clade II stacked bar graph of proportions of samples at each temperature for positive qPCR results (black) and negative qPCR results (white). (F) Clade II stacked bar graph of proportions of samples at each depth for positive qPCR results (black) and negative qPCR results (white). 

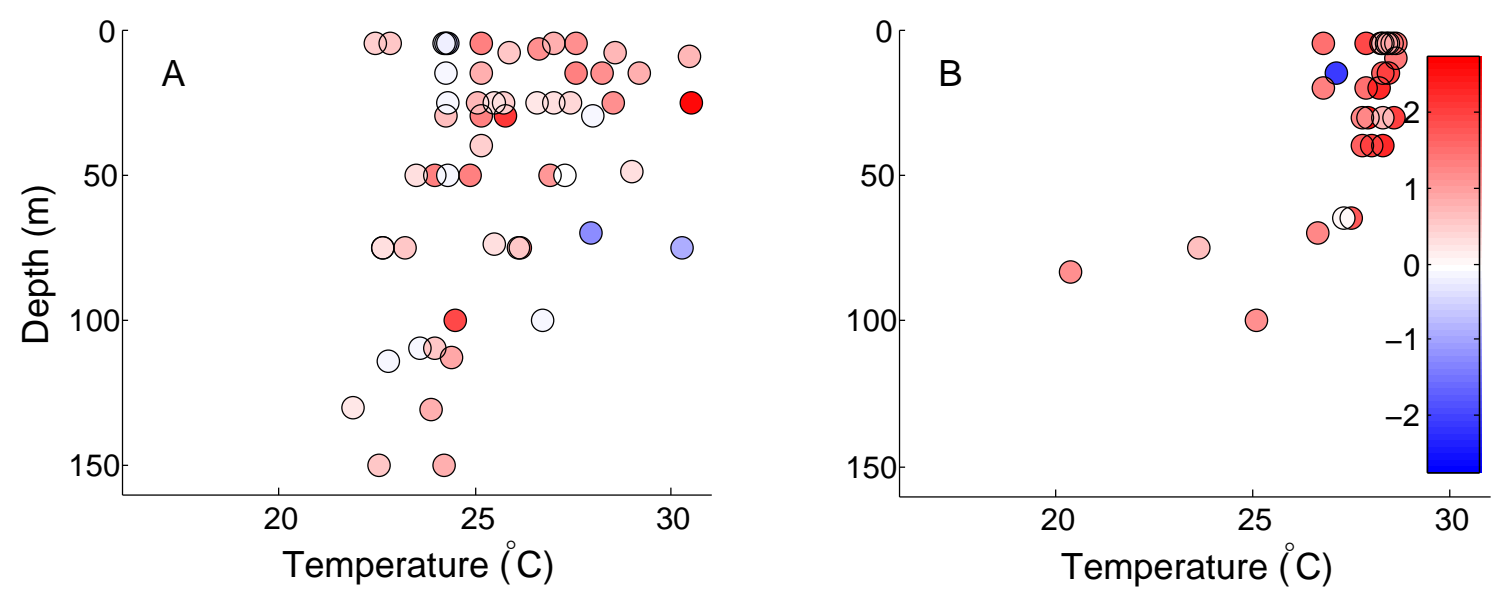

Figure 4-11: Ratio of clade II:clade I concentrations in temperature-depth space. Y-axis represents depth $(\mathrm{m})$ and $\mathrm{x}$-axis represents temperature $\left({ }^{\circ} \mathrm{C}\right)$. Circle color represents $\log _{10}$-transformed ratios of clade II concentrations (copies het $\mathrm{L}^{-1}$ ) to clade I concentrations (copies hetR $\mathrm{L}^{-1}$ ). (A) Pacific samples, (B) Atlantic samples.

The depths of maximum concentration $\left(\mathrm{z}_{\max }\right)$ for clade I were often found deeper as well as shallower than the MLD (Fig. 4-12A, C), while $\mathrm{z}_{\text {max }}$ 's for clade II were typically shallower than the MLD (Fig. 4-12B, D). During the west Pacific Warm pool cruise, the $\mathrm{z}_{\max }$ for all Trichodesmium was mostly in the upper $30 \mathrm{~m}$; however, clade I had more profiles with $\mathrm{z}_{\max }>30 \mathrm{~m}$ than clade II (Fig. 4-13). Several profiles had double Trichodesmium local maxima. For clade I, double maxima were observed in Stas. SJ0609-14, 15, and 23; KM0701-3, 5, 7-10, 13, 18, 19, 21 - 23, and 30; and KM0703-4 (Figs. 4-2A - 4-4A). Double maxima for clade II were observed in Stas. SJ0609-16, 17, and 20; KM0701-3, 16, and 18; and KM0703-11 and 12 (Figs. 4-2B - 4-4B). 

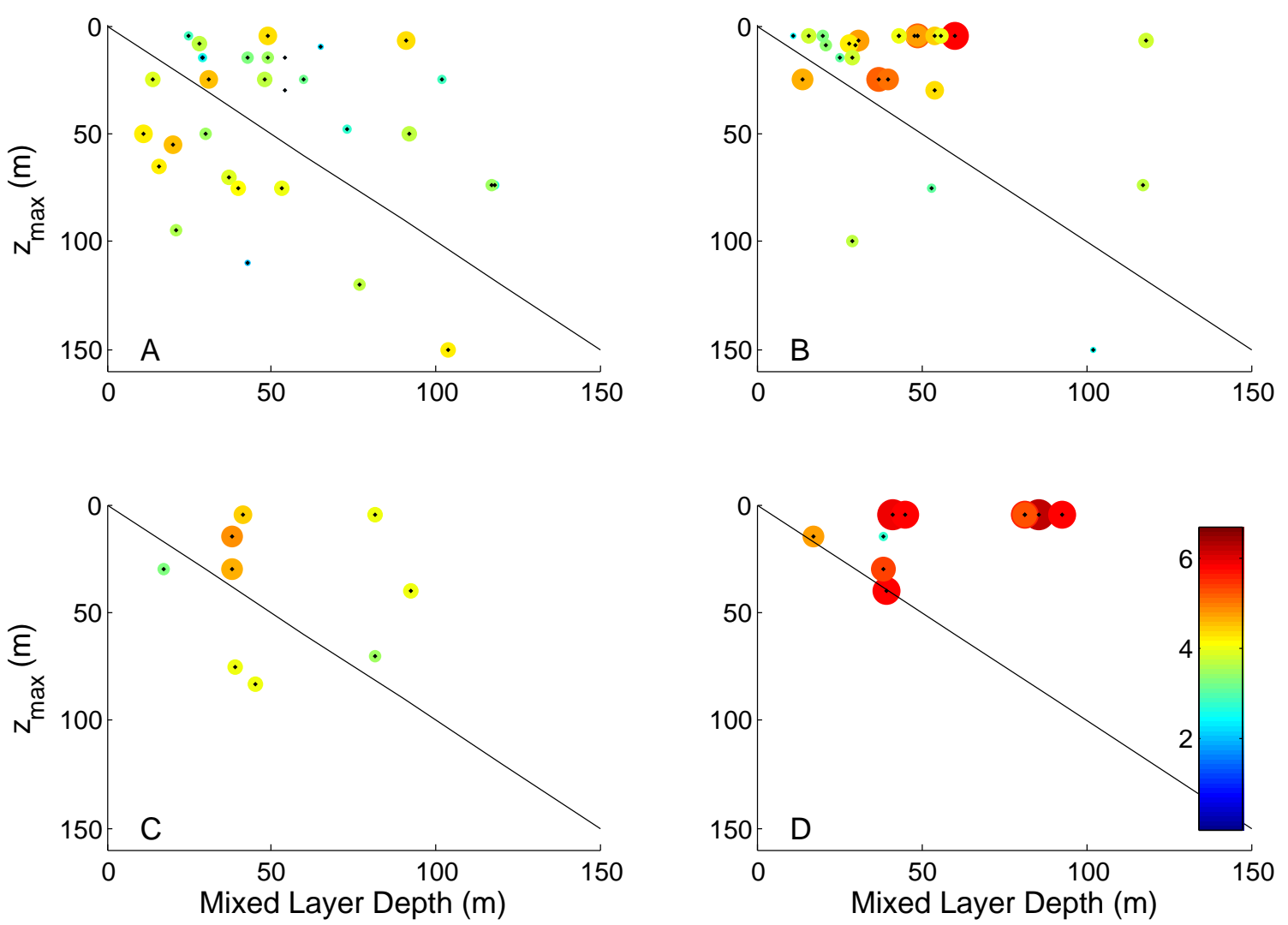

Figure 4-12: Plot of depth of maximum concentration $\left(\mathrm{z}_{\max }\right)$ versus mixed layer depth (MLD), represented by black dots. Circle color and size represent maximum Trichodesmium concentrations for each profile $\left(\log _{10}\left(\operatorname{copies~hetR~} \mathrm{L}^{-1}\right)\right)$. Black line demarcates $\mathrm{Z}_{\text {max }}=$ MLD. (A) Clade I, Pacific samples; (B) clade II, Pacific samples; (C) clade I, Atlantic samples; (D) clade II, Atlantic samples. 

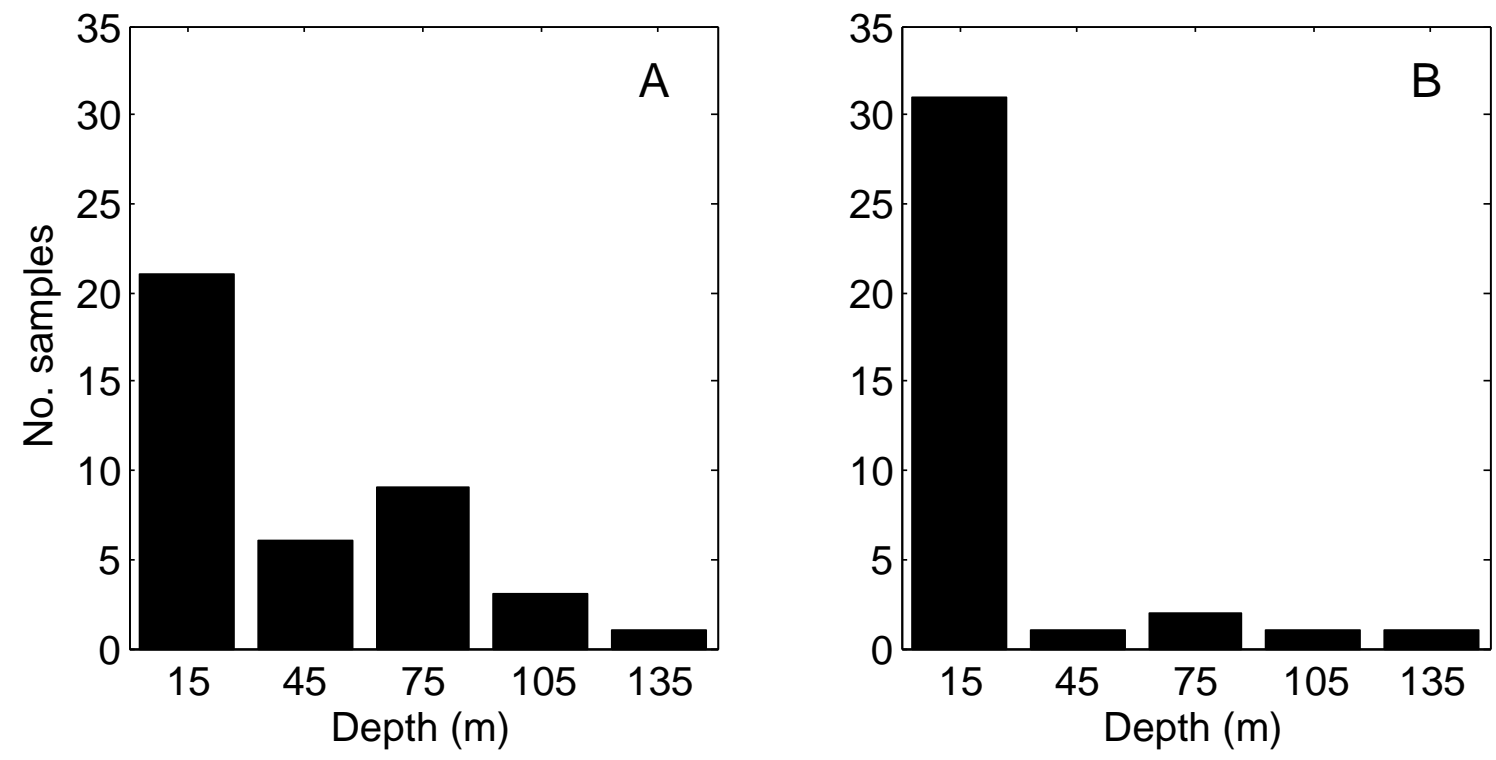

Figure 4-13: Histograms of depth of maximum concentration $\left(\mathrm{z}_{\max }\right)$ for depth profiles from all three transects. (A) Clade I $\mathrm{z}_{\max }$. (B) Clade II $\mathrm{z}_{\max }$. 

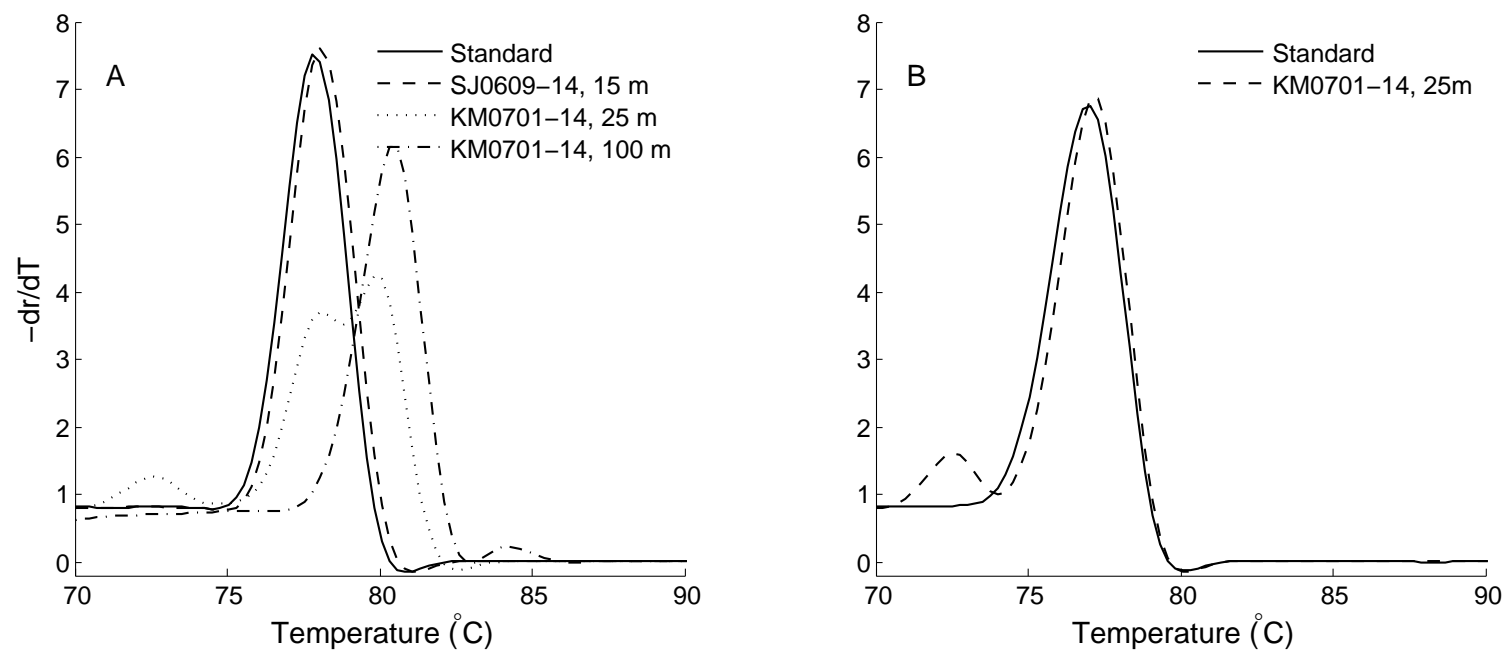

Figure 4-14: Melt curves of qPCR products. (A) Clade I melt curve of a cloned standard and selected field samples. (B) Clade II melt curve of standard and selected field sample.

Melting types, temperature, and depth. Melt curve analysis of qPCR products revealed a variety of melting temperatures $\left(\mathrm{T}_{m}\right)$ of clade I products (Fig. 4-14A). Cloned standards had $\mathrm{T}_{m} \approx 78^{\circ} \mathrm{C}$. Many field samples matched the standard $\mathrm{T}_{m}$ (I-78), but many had double peaks or a single peak with $\mathrm{T}_{m} \approx 80^{\circ} \mathrm{C}$ (I-80). All Clade II field products had melt curves resembling cloned standards with $\mathrm{T}_{m} \approx 78^{\circ} \mathrm{C}$, although many had primer dimers as indicated by a $\mathrm{T}_{m} \approx 73^{\circ} \mathrm{C}$ (Fig. 4-14B). I-78 sequences closely resembled $T$. erythraeum IMS101 while sequences of I-80 closely resembled the T. contortum-T. tenue clade in Janson et al. (1999) (Fig. 4-15A). Some clade II product sequences closely resembled the field sample T. aureum reported in Lundgren et al. (2005) (Fig. 4-15B). I-80 products from the west Pacific warm pool were found in almost the full range of temperature (Fig 4-16C) and were more common in deep than in shallow samples (Fig. 4-16D). 
A
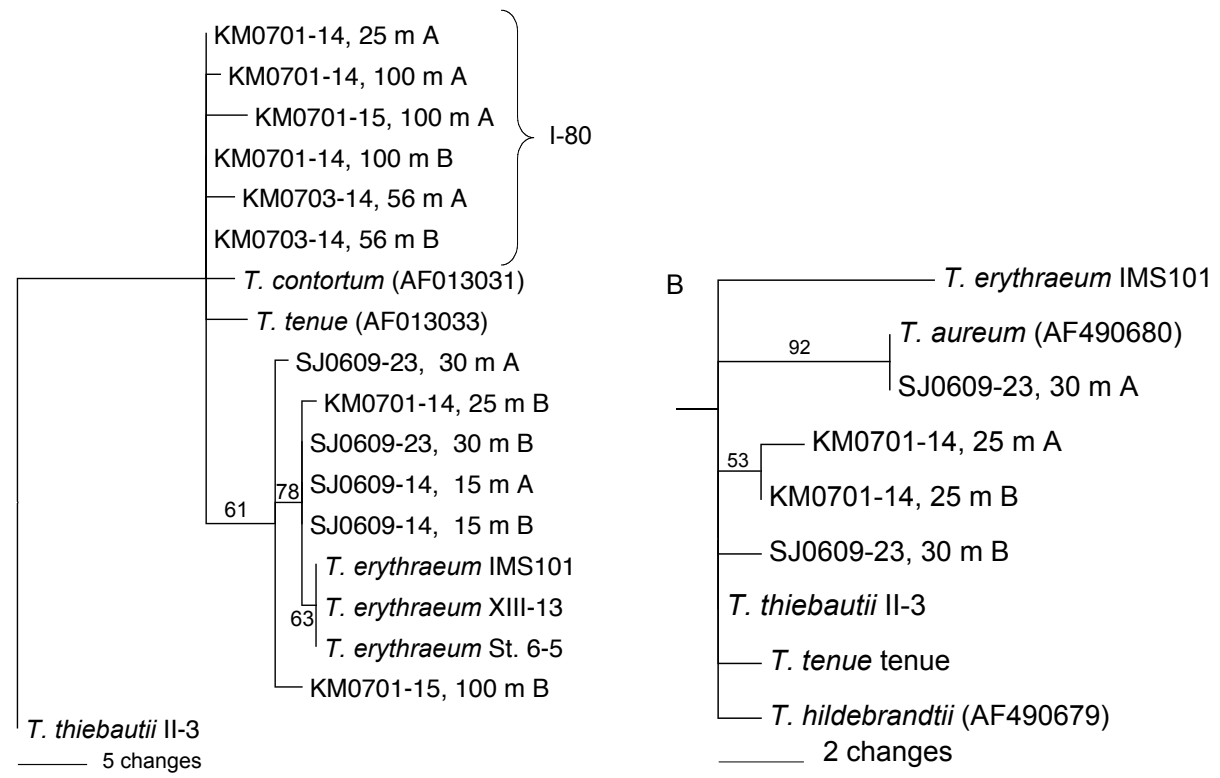

Figure 4-15: Parsimony tree of qPCR products from select field samples, cultured Trichodesmium strains, and published field samples. Each tree was bootstrapped $2000 \times$. (A) Clade I qPCR products, cultured T. erythraeum strains, and field samples from Janson et al. (1999), indicated by GenBank numbers in parentheses. The tree was rooted with $T$. thiebautii II-3. (B) Clade II qPCR products, cultured T. thiebautii strains, and field samples from Lundgren et al. (2005), indicated by GenBank numbers in parentheses. The tree was rooted with T. erythraeum IMS101. 

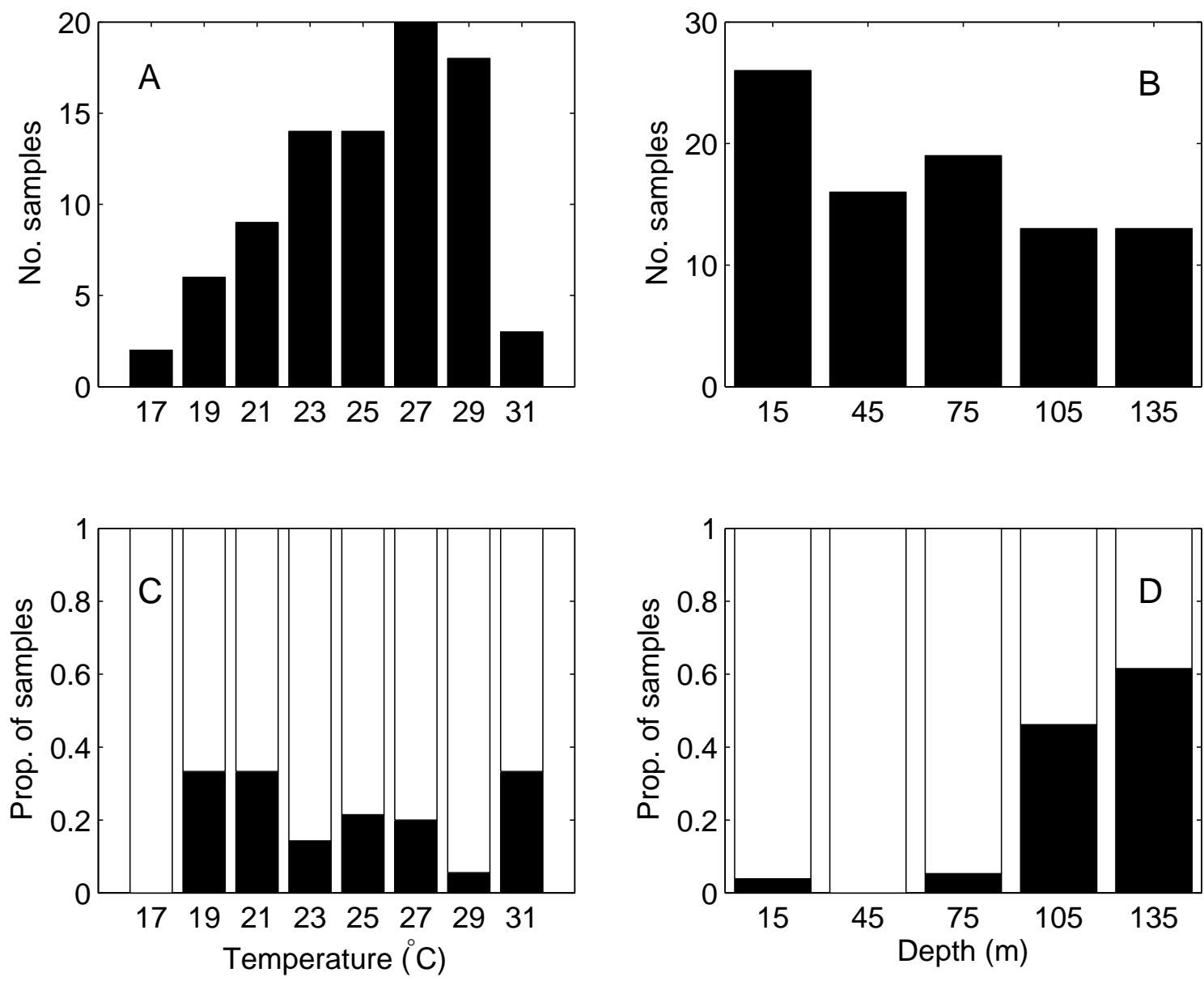

Figure 4-16: Histograms of temperature (A, C) and depth (B, D) for clade I qPCR products of the west Pacific warm pool cruise KM0701, separated by melting temperature $\left(\mathrm{T}_{m}\right)$. (A) Histogram of potential temperature $\left({ }^{\circ} \mathrm{C}\right)$ for qPCR samples with positive results for clade I. (B) Histogram of depth (m) for qPCR samples with positive results for clade I. (C) Stacked bar graph of proportions of samples at each temperature for $\mathrm{T}_{m} \approx 80^{\circ} \mathrm{C}$ (I-80, black) and $\mathrm{T}_{m} \approx 78^{\circ} \mathrm{C}$ (I-78, white). (D) Stacked bar graph of proportions of samples at each depth for I-80 (black) and I-78 (white). I-80 sequences resemble the T. contortum-T. tenue clade of Janson et al. (1999) and I-78 sequences resemble clade I). 
West Pacific warm pool diel cycle. The diel station in the center of the west Pacific warm pool (Sta KM0701-14) was an Eulerian station, fixed in space. The maximum concentration for clade I was $5.4 \times 10^{4}$ copies $h e t R \mathrm{~L}^{-1}$ and for clade II was $4.8 \times 10^{5}$ copies het $\mathrm{L}^{-1}$ (Fig. 4-17A, B). Ratios of non-zero clade II:clade I concentrations ranged from 0.005 to 243.9 with ratios $>1$ near the surface and ratios $<1$ at depth. MLD ranged from 28 - $41 \mathrm{~m}$ with the maximum MLD occurring at about 18:30 (Fig. 4-17C, D). Temperature and salinity remained relatively constant throughout the cycle (Fig. 4-17C, D). Photosynthetically active radiation (PAR) increased after 7:00 $\mathrm{h}$ and decreased after 18:00 h (Fig. 4-17E). During the light period, concentrations of clade II at $100 \mathrm{~m}$ and $150 \mathrm{~m}$ decreased, and increased during the dark period (Fig. 4-17B). This pattern was not as distinct for concentrations of clade I (Fig. 4-17A). Clade I had two populations during the day at $5 \mathrm{~m}$ and $75 \mathrm{~m}$, and one population at $75 \mathrm{~m}$ at night (Fig. 4-17A). As PAR increased, the $\mathrm{z}_{\text {max }}$ for both clades shallowed and then deepened again as PAR decreased (Fig. 4-17E). 


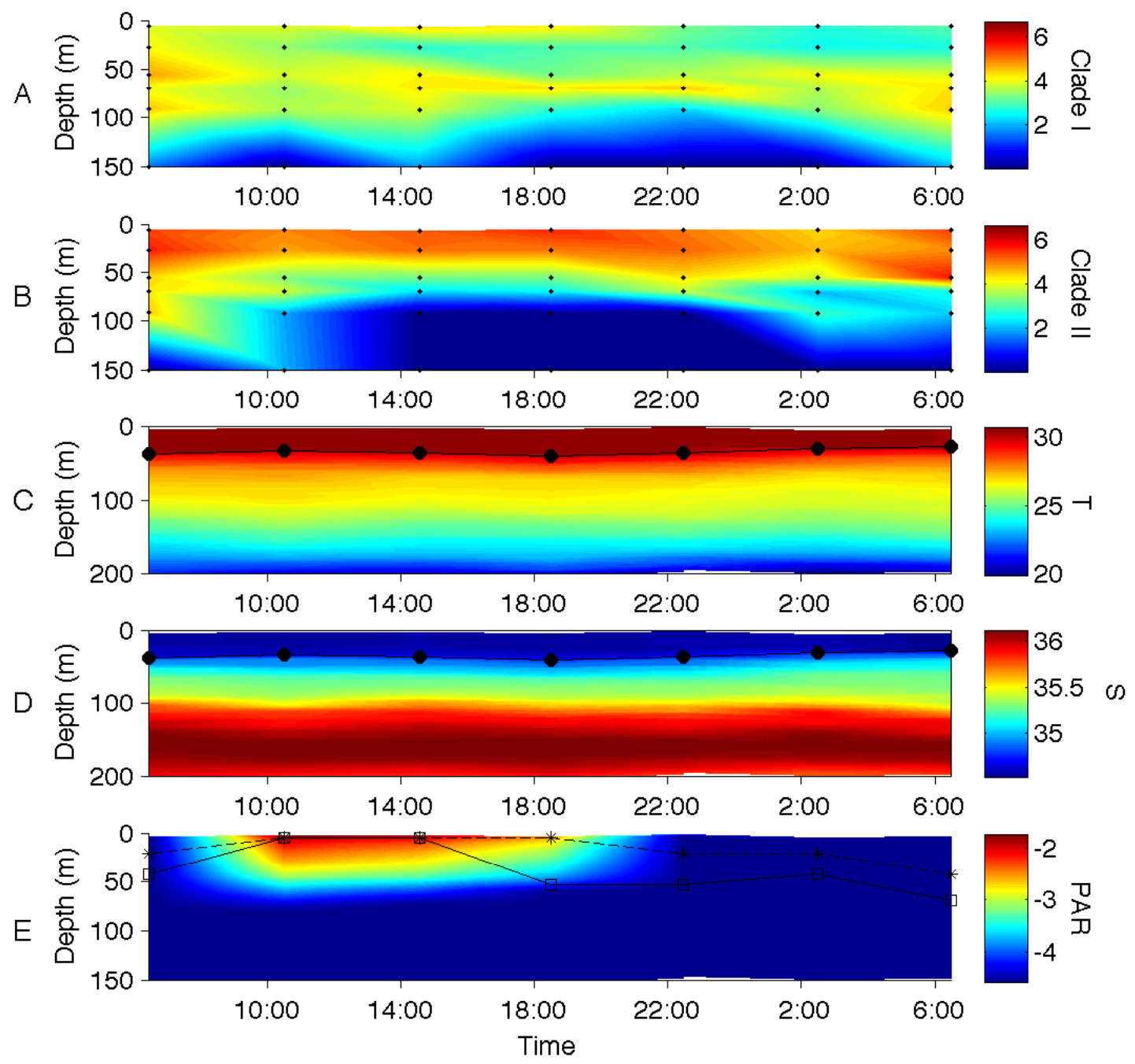

Figure 4-17: Data from the diel cycle at Sta 14 of cruise KM0701. The X-axes represent local time. The y-axes represent depth $(\mathrm{m})$. Black circles connected by a black line indicate mixed layer depth (MLD). (A) Pseudocolor plot of qPCR data for Trichodesmium clade I. Colors represent $\log _{10}$ (copies het $\mathrm{mL}^{-1}$ ). Black dots indicate the location of the qPCR samples. (B) Pseudocolor plot as described in (A) for Trichodesmium clade II. (C) Potential temperature in ${ }^{\circ}$ C. (D) Salinity in PSU. (E) $\log _{10}$ (PAR in $\mu$ mol photons $\mathrm{m}^{-2}$ $\mathrm{s}^{-1}$ ). Clade $\mathrm{I} \mathrm{z}_{\max }$ is indicated by squares connected by a solid line and clade II $\mathrm{z}_{\text {max }}$ is indicated by asterisks and a dashed line. 


\section{Discussion}

This study is the first attempt at quantifying different clades of Trichodesmium using qPCR. Concentrations of the two clades were measured across horizontal and vertical gradients in the Atlantic and Pacific Oceans. The Pacific Ocean has been under-sampled with respect to Trichodesmium (LaRoche and Breitbarth, 2005), and this study provided a snapshot of field populations in the Pacific along a large horizontal gradient. Concentrations of clade II were higher than those of clade I in all three transects, and both clades reached higher concentrations in the Atlantic than in the Pacific. Where both clades were found, clade II was up to two orders of magnitude greater than clade I. On the rare occasion that clade I was more dominant, it was up to two orders of magnitude greater than clade II. The correlation found between clade I and clade II concentrations was driven by samples from the west Pacific warm pool. Concentrations of the two clades were correlated in the Pacific Ocean, indicating that they may be limited by the same factors where they coexist in the Pacific Ocean. In contrast, the two clades were not correlated in the Equatorial Atlantic. The disparate distributions in the Atlantic indicate that the two clades were limited by different factors.

The relationship between cell counts and qPCR results from the Equatorial Atlantic was not one-to-one, but the linear fit of the $\log _{10}$ transformed data was within the range found in cultured strains (Chapt. 3). Cyanobacteria are known to contain several genome copies per cell (Cohen and Gurevitz, 2006). For example, the filamentous, heterocystous cyanobacteria Anabaena spp. have been estimated to have from five to eight copies per cell in Anabaena variabilis (Chapman and Meeks, 1987) and up to 10 - 20 copies per cell in Anabaena sp. PCC 7120 (Herdman et al., 1979; Sakr et al., 2006). Estimating cell numbers in Trichodesmium colonies is not trivial given the diversity of colony diameter and filament density within a colony. Cell counts within dense colonies can be greatly underestimated using microscopy. Multiple genome copies per cell and underestimation of cells within colonies can explain the 1-2 orders of magnitude difference 
between cell counts and copies of hetR per volume.

Trichodesmium spp. are able to fulfill their need for $\mathrm{N}$ through $\mathrm{N}_{2}$ fixation, so they tend to be limited by other nutrients such as $\mathrm{P}$ and Fe. I expected to see a correlation between nutrients and Trichodesmium concentrations. However, there was no correlation found between concentrations of Trichodesmium and DIP or Fe, for neither individual transects nor the combined data. In the case of $\mathrm{P}$, Trichodesmium spp. are able to access dissolved organic phosphorus (DOP) such as phosphomonoesters (Sohm and Capone, 2006; Mather et al., 2008) and phosphonates (Dyhrman et al., 2006). While populations may show P stress, cells can alleviate P limitation by the use of DOP instead of DIP. While DIP concentrations were lower in the Atlantic than in the Pacific in the study, populations of Trichodesmium in the Atlantic may be obtaining their P from organic sources. Measurements of Fe had the same range in both the Atlantic and the Pacific (Chappell, 2009), but we don't know the flux of Fe from sources to each region or the amount of Fe storage in Trichodesmium cells. Aeolian input of Fe from dust is greater in the Atlantic than the Pacific Ocean (Wu et al., 2000; Moore and Braucher, 2008), but input of Fe from continental margins is important in both regions (Moore and Braucher, 2008). Stas. KM0701-17 and 18 were close to the Vanuatu Islands, and Sta. KM0701-19 was close to New Caledonia. Chappell (2009) found that Sta. KM0701-17 had high dissolved Fe, possibly due to advection of Fe from Aniwa Island of the Vanuatu archipelago. The potential of these islands as sources of nutrients and their influence on Trichodesmium populations cannot be ignored. Nutrient history and cell quotas may be more important than environmental nutrient concentrations to the growth of Trichodesmium cells, as ambient nutrient concentrations may not reflect the nutrient status of the cells.

Nutrient limitation can define phytoplankton niches. According to classic resource competition theory, species with different limiting resources can coexist (Tilman, 1977). Coexistence of the two clades of Trichodesmium could be possible through differential limitation by $\mathrm{P}$ and Fe. The highest clade II:clade I ratios were found in areas of low DIP, which is consistent with the hypothesis that clade I may be more limited by P while clade 
II may be more limited by Fe. For example, Pacific Sta. KM0701-10 had the highest [DIP] of the transect, high clade I concentration, and no amplification of clade II. However, the majority of the low DIP stations were in the Atlantic, and Atlantic concentrations of clade I as well as clade II exceeded those of the Pacific. Factors that differ between the Atlantic and the Pacific other than nutrients might be influencing Trichodesmium distributions. Niche differentiation of Trichodesmium spp. is not as simple as differential nutrient limitation, but further investigations are required to understand the influence $\mathrm{P}$ and $\mathrm{Fe}$ have on the growth and distributions of different Trichodesmium spp.

While there was not a distinct spatial separation between the clades of Trichodesmium, clade I populations had colder and deeper ranges than clade II. The presence of Trichodesmium cells in deep or cold waters does not necessarily indicate growth under those conditions; cells may have been advected there. However, these temperature and depth ranges may indicate a higher tolerance for low light and cold conditions in clade I than in clade II. Chappell (2009) found that while cultured strains of clade I had higher optimum temperatures $\left(28^{\circ} \mathrm{C}\right)$ than a clade II strain $\left(26^{\circ} \mathrm{C}\right)$, the clade I strains had higher growth rates across all temperatures measured. Clade I had both surface and deep populations, discernible by $\mathrm{T}_{m}$ as I-78 and I-80. I-80 may be the $T$. contortum - $T$. tenue group sequenced in Janson et al. (1999). Clade II products also had sequences similar to T. aureum described in Lundgren et al. (2005). The range of depth for clade I may be driven by the I-80 population, which was the prominent melting type of clade I at depth. The T. tenue in Janson et al. (1999) was collected from the central Atlantic Ocean at $75 \mathrm{~m}$, while our culturing efforts have been mostly focused at the surface. Culturing efforts must extend deeper in order to capture the full diversity of Trichodesmium spp. Having cultured representatives of these deep clades would enable the development of more specific qPCR primers. The I-80 population did not have a particularly colder range than I-78, so the depth distribution of I-80 may be driven by light rather than temperature. Depth analysis of I-80 could be done only on the KM0701 profiles, which were sampled consistently to $150 \mathrm{~m}$. Even at $150 \mathrm{~m}$, the bottom of the Trichodesmium layer was not always reached. 
In addition, distributions of Trichodesmium populations are patchy. Future studies should aim for higher resolution and deeper samples than this or previous studies, for example using the VPR in concert with qPCR.

The deep range of clade I was surprising. Cultured strains of clade I tend to be highly buoyant. In field populations, tuft colonies, which are typically associated with $T$. erythraeum, were found to be concentrated at the surface while puff colonies, presumably T. thiebautii, were distributed at depth (Post et al., 2002; Davis and McGillicuddy, 2006). With the exception of Sta. KM0701-10, the majority of colonies during the Equatorial Atlantic and west Pacific warm pool cruises, both puffs and tufts, belonged to clade II (Chappell, 2009). Most of the biomass of clade I during this study was probably in the form of single trichomes, which would not have the same buoyancy as colonies and would not be detected using the VPR (Davis and McGillicuddy, 2006). Composition of the photosynthetic accessory pigment phycoerythrin (PE) of the two strains also predicts clade I to be a surface-dweller and clade II to be depth-adapted. Cultured strains of clade I had high amounts of the PE subcomponent phycoerythrobilin (PEB), and strains of clade II had high amounts of the subcomponent phycourobilin (PUB) (Chapt. 2). PEB absorbs green wavelengths of light (around $545 \mathrm{~nm}$ ) while PUB absorbs blue-green light (around $495 \mathrm{~nm}$ ). Since blue wavelengths penetrate deeper in the water column than green wavelengths, PUB should be the preferred pigment at depth and clade II should be more depthadapted. However, clade I cells have higher PE content than clade II (Carpenter et al., 1993), which might be an adaptation to low light. The T. contortum described by Janson et al. (1999) was straw-colored while the T. tenue described was deeply pigmented, so the pigmentation of the deep I- 80 population of clade I might have been diverse. Clade II distributions indicate a high light requirement or at least a preference for shallow waters. The $\mathrm{z}_{\max }$ 's for clade II were consistently shallower than the MLD. During the west Pacific warm pool cruise, clade II was not found in the warm but deep mixed layers of stations north of the Equator, but was found in the cool but shallow mixed layer of the southernmost end of the transect. 
The difference in Trichodesmium clade concentrations might be due to differential mortality. Clade II is known to produce more toxins than clade I, and clade I is preferentially grazed by the harpacticoid copepod Macrosetella gracilis (O'Neil and Roman, 1994). If grazing mortality is significant, standing stocks of clade I could be lower than clade II even if clade I growth is higher. Virus-like particles (VLP) found in clade II field samples from the North Pacific were larger than the VLP found in clade I (Hewson et al., 2004), but rates of viral lysis for the two clades are not known. Autolysis in the two clades might be triggered by different stressors or ranges of stressors such as light or nutrient levels.

During the diel cycle at KM0701-14, depth distributions of Trichodesmium were more shallow at daytime than nighttime. The effect was much more noticeable in clade II populations than in clade I. This was an Eulerian station, so the populations sampled were not from the same water mass, and we cannot ignore the possibility that the nighttime shallowing pattern observed may be from the advection of a water mass with different properties and populations rather than a response to a diel cycle. However, the possible movement of Trichodesmium from the surface to below the phosphocline at night would support the P mining hypothesis (Karl et al., 1992; White et al., 2006b).

Most of the information available about the physiology of Trichodesmium is based on clade I, particularly T. erythraeum IMS101. Culture studies investigating Trichodesmium physiology in response to nutrients (Mulholland et al., 2002; Holl and Montoya, 2005), trace metals (Tuit et al., 2004), $\mathrm{pCO}_{2}$ (Hutchins et al., 2007), light (Bell and Fu, 2005), and temperature (Breitbarth et al., 2007) have all been based on cultures of T. erythraeum. Consequently, models investigating $\mathrm{N}_{2}$ fixation incorporate parameters based on T. erythraeum (Moore et al., 2004; Rabouille et al., 2006; Goebel et al., 2007). In this study, clade I, which includes T. erythraeum, has been shown to be less prominent than clade II. If the physiology of clade I and clade II are significantly different, our understanding of global $\mathrm{N}_{2}$ fixation based on parameters from clade I may be skewed. This study has taken a step toward examining niche differentiation in Trichodesmium and getting a better 
understanding of the global distribution of phylotypes of this important $\mathrm{N}_{2}$ fixer.

Acknowledgements: I would like to thank R. Gast and her laboratory for allowing me to use their beadbeater; the S. Chisholm and E. DeLong laboratories for allowing me to use their LightCycler 480 and liquid handler, particularly R. Malmstrom for being generous with his time and teaching me how to use them; the J. Zehr laboratory, notably K. Achilles, I. Hewson, and R. Paerl for providing me with the cell count slides from SJ0609; R. Horak for providing nutrient data from KM0703; E. Orchard for helping me to acquire the KM0703 data; P. D. Chappell for graciously sharing her Fe data, E. Webb and P. D. Chappell for providing me with nutrient data from SJ0609 and KM0701; W. Krey and E. Webb for acquiring qPCR samples for me during the second leg of KM0701; J. Waterbury for acquiring qPCR samples for me from SJ0609 and KM0703; and S. Cooley, S. Doney, and D. Glover for their advice on plotting transect data. I would like to thank T. Mincer for his advice on overcoming qPCR inhibition, and S. Doney and J. Waterbury for their insight in analyzing this data. I would like to express my gratitude to the captain, crews, and named chief scientists of the following cruises: Z. Johnson and E. Zinser of the R/V Kilo Moana cruise KM0701, and J. Montoya and J. Zehr of the R/V Seward Johnson cruise SJ0609 and R/V Kilo Moana cruise KM0703. This work was supported by the Woods Hole Oceanographic Institution (WHOI) Ocean Life Institute (OLI) grant to J. Waterbury and the Center for Microbial Oceanography Research and Education (CMORE), an NSF Science and Technology Center (EF-0424599). 


\section{CHAPTER 5}

\section{Cross-basin comparison of phosphorus stress and nitrogen fixation in Trichodesmium}

This manuscript has been published in Limnology and Oceanography as:

Annette M. Hynes, P. Dreux Chappell, Sonya T. Dyhrman, Scott C. Doney, and Eric A.

Webb. 2009. Cross-basin comparison of phosphorus stress and nitrogen fixation in Trichodesmium, Limnol. Oceanogr. 54(5):1438 - 1448.

Copyright (2009) by the American Society of Limnology and Oceanography, Inc. Figures and text are reprinted here with permission.

\section{ABSTRACT:}

We investigated the phosphorus (P) status and $\mathrm{N}_{2}$ fixation rates of Trichodesmium populations from the North Pacific, western South Pacific, and western North Atlantic. Colonies of Trichodesmium were collected and analyzed for endogenous alkaline phosphatase (AP) activity using enzyme-labeled fluorescence (ELF) and for nitrogenase activity using acetylene reduction. AP hydrolyzes dissolved inorganic phosphate (DIP) from dissolved organic phosphorus (DOP) and is active in Trichodesmium colonies experiencing P stress. Across multiple stations in the subtropical North and South Pacific, there was low to moderate ELF labeling in Trichodesmium, although labeling was present in other taxa. In contrast, Trichodesmium ELF labeling in the North Atlantic ranged from 
low to high. Low ELF labeling corresponded with high DIP concentrations while high ELF labeling occurred only at North Atlantic stations with DIP concentrations $\leq 40 \mathrm{nmol}$ $\mathrm{L}^{-1}$, indicating that Trichodesmium was not experiencing dramatic $\mathrm{P}$ stress in the Pacific Ocean while P stress was evident in the western North Atlantic. However, nitrogenase activity was significantly higher in the P-stressed western North Atlantic than in the Pacific Ocean (0.40 - 1.30 compared to $0.01-0.46 \mathrm{nmol} \mathrm{C}_{2} \mathrm{H}_{4} \mathrm{~h}^{-1}$ colony ${ }^{-1}$ ). These data underscore the differential basin-level importance of $\mathrm{P}$ availability to Trichodesmium and suggest that factors other than $\mathrm{P}$ are constraining their $\mathrm{N}_{2}$ fixation rates in the Pacific.

\section{Introduction}

New nitrogen. Fixed nitrogen $(\mathrm{N})$ is considered to be the proximal limiting nutrient in ocean ecosystems. For a system in steady-state, new production is the excess production available for export and is a function of new $\mathrm{N}$ entering the system and stimulating production (Eppley and Peterson, 1979). New N comes from eddy diffusion processes, seasonal deep mixing, atmospheric deposition, lateral advection, and $\mathrm{N}_{2}$ fixation by diazotrophs (Dugdale and Goering, 1967). Upwelled water from the deep ocean contains both elevated dissolved inorganic nitrogen (DIN) and dissolved inorganic carbon (DIC), so export production from upwelled DIN does not generally result in a significant net removal of DIC from surface water. However, DIN from $\mathrm{N}_{2}$ fixation is not related to the DIC supply from deep water and can drive uptake of $\mathrm{CO}_{2}$ and export production (Hood et al., 2000). In global marine models, the presence of $\mathrm{N}_{2}$-fixers increased primary production by diatoms and small phytoplankton through the excretion of dissolved organic nitrogen (DON) in N-limited areas (Moore et al., 2004). In the subtropical Atlantic, model results show that $\mathrm{N}_{2}$ fixation increases DIN, phytoplankton biomass, primary production, and export flux (Coles et al., 2004).

The cyanobacterium Trichodesmium is an important source of new $\mathrm{N}$ in tropical and subtropical oceans (Capone et al., 1997). In the North Atlantic, $\mathrm{N}_{2}$ fixation rates of Trichodesmium can equal or exceed the vertical flux of nitrate into surface waters (Capone 
et al., 2005), while in the North Pacific, $\mathrm{N}_{2}$ fixation is the source of up to half of the new N (Karl et al., 1997). Global ecosystem models use parameters from Trichodesmium to model $\mathrm{N}_{2}$ fixation (Coles et al., 2004; Moore et al., 2004; Moore and Doney, 2007). Elucidating the factors limiting the growth and $\mathrm{N}_{2}$ fixation rates of Trichodesmium is vital to understanding the $\mathrm{N}$ cycle and its link to the carbon cycle.

Phosphorus and iron. $\mathrm{N}_{2}$ fixation by Trichodesmium can be limited by a variety of factors including light, temperature, mixed layer depth, dissolved oxygen, and nutrients such as phosphorus (P) and iron (Fe) (Wu et al., 2000; Moore et al., 2004). Like N, new P can reach the surface by mixing, upwelling from deep waters, lateral advection, or deposition; however, there is no process analogous to $\mathrm{N}_{2}$ fixation to act as a source for bioavailable $\mathrm{P}$ (Karl, 2002). Nitrogenase, the enzyme responsible for $\mathrm{N}_{2}$ fixation, requires multiple Fe atoms per molecule. Sources of Fe to open-ocean environments are dust deposition, advection from continental margins, and upwelling (Fung et al., 2000). Due to these obligate requirements and low oceanic concentrations, $\mathrm{P}$ and $\mathrm{Fe}$ bioavailability may influence the growth and $\mathrm{N}_{2}$ fixation rates of Trichodesmium field populations (Berman-Frank et al., 2001; Sañudo-Wilhelmy et al., 2001). In the central Atlantic, $\mathrm{N}_{2}$ fixation positively correlated to the $\mathrm{P}$ content of Trichodesmium (Sañudo-Wilhelmy et al., 2001) while in the eastern tropical Atlantic, $\mathrm{N}_{2}$ fixation was co-limited by $\mathrm{P}$ and Fe (Mills et al., 2004). In the North Pacific Subtropical Gyre (NPSG), P supply may be the ultimate control on new and export production at Sta. ALOHA (Karl et al., 1997), but Fe rather than P is likely to limit $\mathrm{N}_{2}$ fixation (Wu et al., 2000).

While subtropical gyres of both the North Pacific and the North Atlantic are oligotrophic, the NPSG has higher dissolved inorganic phosphate (DIP) concentrations: 3.0 - $191.3 \mathrm{nmol} \mathrm{L}{ }^{-1}$ in the NPSG compared to $0.2-1.0 \mathrm{nmol} \mathrm{L}^{-1}$ in the North Atlantic (Hawaii Ocean Time-series (HOT), http://hahana.soest.hawaii.edu/hot/hot_jgofs.html; (Wu et al., 2000). Dissolved organic and particulate pools of $\mathrm{P}$ are also higher in the NPSG than the North Atlantic (Ammerman et al., 2003). In the western South Pacific, 
DIP concentrations ranged from 80 to $218 \mathrm{nmol} \mathrm{L}^{-1}$ in oceanic regions (Campbell et al., 2005) and from < 30 to 63 nmol L ${ }^{-1}$ near New Caledonia (Van Den Broeck et al., 2004). P limitation may be related to inputs of Fe (Wu et al., 2000). The NPSG has lower aeolian Fe input than the subtropical North Atlantic: $0.08-0.16 \mu \mathrm{mol} \mathrm{Fe} \mathrm{m}{ }^{-2} \mathrm{~d}^{-1}$ compared to $0.2-0.8 \mu \mathrm{mol} \mathrm{Fe} \mathrm{m}^{-2} \mathrm{~d}^{-1}$, respectively (Wu et al., 2000). Open ocean dissolved Fe concentrations in the upper $100 \mathrm{~m}$ averaged $720 \mathrm{pmol} \mathrm{L}^{-1}$ in the North Atlantic, 200 pmol L ${ }^{-1}$ in the North Pacific, and $110 \mathrm{pmol} \mathrm{L}^{-1}$ in the Equatorial Pacific (Moore and Braucher, 2008). During a cruise in the western South Pacific, open ocean dissolved Fe concentrations ranged from $<60$ to $760 \mathrm{pmol} \mathrm{L}^{-1}$ while coastal dissolved Fe concentrations ranged from $<60$ to $1000 \mathrm{pmol} \mathrm{L}^{-1}$ (Campbell et al., 2005). In addition to low concentrations, bioavailability of $\mathrm{Fe}$ and $\mathrm{P}$ can limit abundance and activity of Trichodesmium, and differences in the availability of these nutrients across ocean basins create a variety of limitation scenarios.

Alkaline phosphatase. Herein we define nutrient stress as the exposure of a cell to low inorganic nutrient conditions which stimulates a response from the cell. Phytoplankton can launch a physiological response under conditions of $\mathrm{P}$ stress to ameliorate potential limitation. DIP is the most readily available form of phosphorus. The utilization of dissolved organic phosphorus (DOP) can prevent limitation of Trichodesmium growth and $\mathrm{N}_{2}$ fixation rates in low DIP environments. However, accessing the DOP pool requires the cell to invest energetic, carbon, and nitrogen resources in enzymes such as alkaline phosphatase (AP). We define this as distinct from $\mathrm{P}$ limitation, which is the reduction of either growth or $\mathrm{N}_{2}$ fixation rates as a result of persistent stress. AP hydrolyzes DIP from DOP and is commonly induced in phytoplankton species experiencing P stress (Stihl et al., 2001; Dyhrman et al., 2002). DOP is likely a significant source of P for Trichodesmium spp. (Sohm and Capone, 2006; Mather et al., 2008), and the presence of AP activity can indicate Trichodesmium field populations responding to low levels of DIP (Stihl et al., 2001). AP activity can be measured using colorimetric and fluorometric assays or de- 
tected through molecular diagnostic tools. Enzyme-labeled fluorescence (ELF) is a commercially available, cell-specific molecular diagnostic tool that detects AP activity. The phosphatase substrate precipitates when cleaved by AP and fluoresces green under ultraviolet light (González-Gil et al., 1998). In the laboratory, P-depleted Trichodesmium erythraeum IMS101 batch cultures were ELF-labeled while replete cultures were not (Dyhrman et al., 2002). In ship-board incubations, addition of DIP to ELF-positive populations of Trichodesmium reduced ELF labeling within $48 \mathrm{~h}$ (Webb et al., 2007). We believe that ELF labeling of P-stressed Trichodesmium gives valuable insight into the in situ physiological status of the cells.

In this study we compare Trichodesmium $\mathrm{P}$ stress and nitrogenase activity among tropical and subtropical regions of the western North Atlantic, the North Pacific, and the western South Pacific (Fig. 5-1). We collected colonies of Trichodesmium from four cruises and analyzed endogenous AP activity using ELF, measured nitrogenase activity using acetylene reduction, and sampled surface water nutrients to assess the P status of Trichodesmium populations. Our results show that populations in the western North Atlantic were more $\mathrm{P}$ stressed but had higher nitrogenase activities than populations in the North and South Pacific.

\section{Methods}

Hydrological context. Samples were collected along a north-south transect of the Pacific Ocean as part of the Western Pacific Warm Pool cruise $\left(\mathrm{WP}^{2}\right.$, KM0701) on the R/V Kilo Moana in January-February 2007 (Fig. 5-1A). As part of the Marine Nitrogen Transformation (MANTRA) Biocomplexity Project, samples were taken from south of Hawaii during MP09 in August 2003, on the R/V Revelle (Fig. 5-1B). North Atlantic field samples were collected near the Bermuda Atlantic Time-series Station (BATS) in June and October 2001, aboard the R/V Endeavor (EN355 and EN361, respectively) (Fig. 5-1C). Temperature, salinity, pressure, and density data from each cruise was obtained from a CTD system. Mixed layer depths (MLD) were estimated from potential density profiles. 

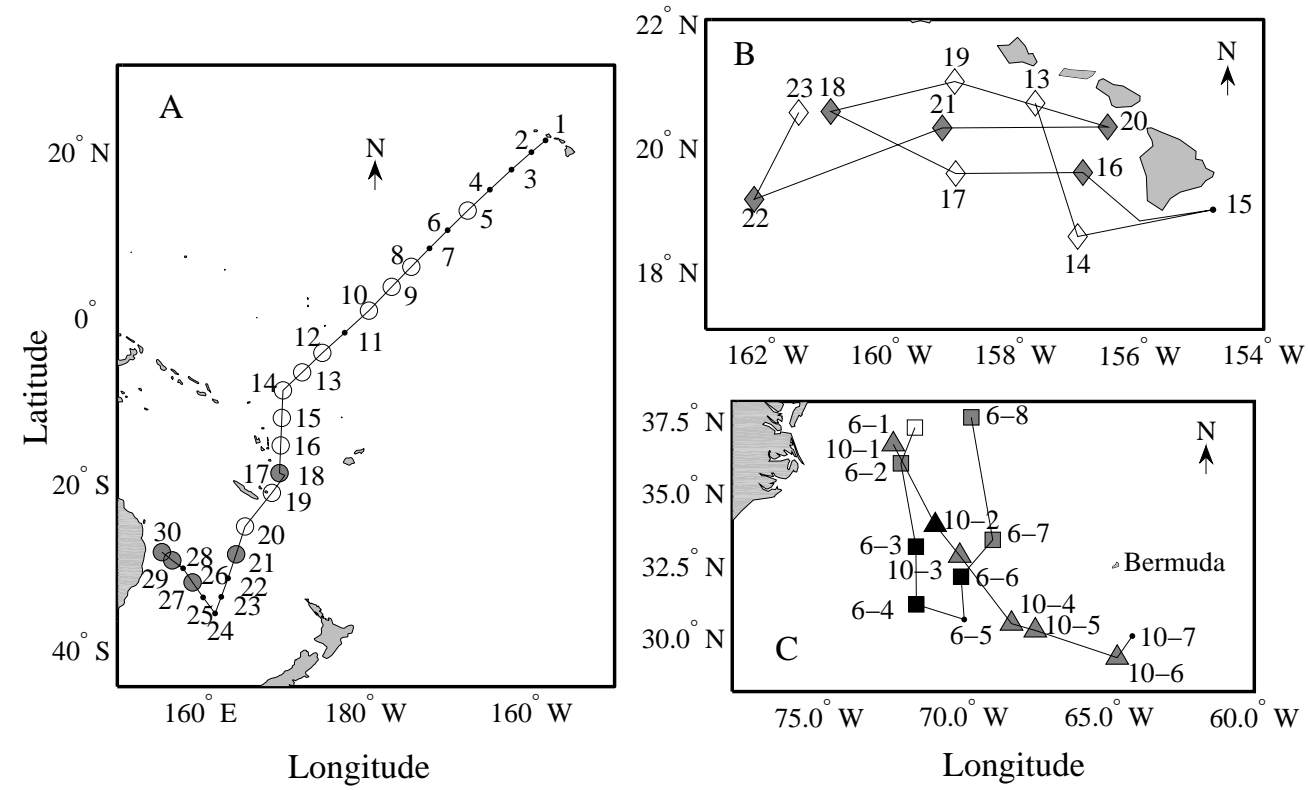

Figure 5-1: Maps of cruise tracks: (A) KM0701 (circles) during January and February 2007, across the west Pacific warm pool; (B) MP09 (diamonds) during August 2003, in the North Pacific; (C) EN355 (squares) during June 2001, and EN361 (triangles) during October 2001, in the western North Atlantic. The highest level of ELF labeling for all colony types at each station is indicated by color: black symbols $=$ ' ++ ', gray symbols $=$ '+ -', open symbols = '- -', small dots = no ELF data.

Sea surface temperature (SST) and salinity were obtained from the ships' underway data.

Collection of colonies. A $0.5 \mathrm{~m}$ diameter, 130.0- $\mu \mathrm{m}$ mesh phytoplankton net with a 30$\mathrm{m}$ line was hand towed for 10 - 20 minutes. Tows for ELF were typically done in the morning while tows for nitrogenase activity were done around noon, the peak of $\mathrm{N}_{2}$ fixation in Trichodesmium, targeting local times of 11:00 h, 12:00 h, and 13:00 h. Specimens were immediately shaded and taken into an air-conditioned laboratory. Colonies of Trichodesmium spp. were collected with polyethylene bulb pipets or plastic bacteriological transfer loops (Fisher Scientific); separated by colony morphology into spherical 'puffs,' fusiform 'tufts,' and occasionally 'bowties'; and washed four times in sterilized seawa- 
ter $(0.2-0.4 \mu \mathrm{m}$-filtered and microwaved to a rolling boil). The colonies were processed immediately for AP or nitrogenase activity. Stations with no data reported had too little Trichodesmium biomass to run the assays.

Enzyme-labeled fluorescence (ELF). The presence of AP activity was determined using the ELF 97 Endogenous Phosphatase Detection Kit (Molecular Probes-Invitrogen) following the improved protocol described in Dyhrman et al. (2002) for two colonies of each morphology. The slides were stored damp and in the dark at $4^{\circ} \mathrm{C}$. ELF slides were viewed with epifluorescent microscopy using a 4, 6-diamidino-2-phenyl-indole (DAPI) long-pass filter set $(360 \mathrm{~nm})$. The entire area of the slide was examined. Digital pictures of Trichodesmium from MP09 and the Atlantic cruises were taken at 200X using a Zeiss Axioplan2 microscope equipped with a Zeiss Axiocam digital camera and Openlab software (Improvision). Digital pictures from KM0701 were taken at 400X using a Zeiss Axiostar microscope equipped with a MRC5 Axiocam and Axiovision software (Zeiss). Samples were rated '- -' for 'no labeling,' ‘+ -' for 'some labeling' if many filaments showed moderate labeling but were not wholly covered or if there was mixture of labeled and unlabeled filaments, and ' ++ ' for 'high labeling' if many filaments showed dense labeling and/or were wholly covered. For the North Atlantic samples, multiple slides were made for each colony type so one rating was given based on all the slides. Filament color was noted as 'normal' if the orange color of the phycoerythrin autofluorescence was visible or 'dim' if the filaments appeared abnormally dark, green or blue. Digital pictures of pennate diatoms associated with Trichodesmium colonies were taken at 400X with the DAPI long-pass filter and a chlorophyll filter (660 nm) using AxioVision 4.2 software.

Nutrient Analysis. For the North and South Pacific (KM0701) and the North Atlantic (EN355 and EN361), surface water was collected using either niskin bottles from a rosette or Go-Flo bottles, filtered through $0.4 \mu \mathrm{m}$ acid-washed polycarbonate filters, and frozen. Samples for KM0701 were sent to the College of Oceanic and Atmospheric Sciences, Oregon State University and DIP was analyzed using a Technicon AutoAnalyzer II by 
J. Jennings with a detection level of $6 \mathrm{nmol} \mathrm{L}^{-1}$. North Atlantic samples were sent to the Ocean Data Facility of the Scripps Institution of Oceanography (http://sts.ucsd.edu/sts/chem) for analysis by a Skalar San Plus autoanalyzer with a detection level of $3 \mathrm{nmol} \mathrm{L}^{-1}$. DIP concentrations for the North Pacific during MP09 were provided by K. Björkman (pers. comm.). Two-way analysis of variance (ANOVA) Tukey-Kramer tests were used to determine significant differences in DIP concentrations among ELF ratings and oceans for stations with both DIP concentrations and ELF samples $(\alpha=0.05)$. DIP concentrations below detection level (BDL) were considered to be 0 in the calculations.

Nitrogenase activity. Nitrogenase activity was assayed during KM0701 and the Atlantic cruises by the acetylene reduction assay described by Capone and Montoya (2001). Five to twenty colonies were placed in $20-30 \mathrm{~mL}$ of $0.2 \mu \mathrm{m}$-filtered sea water in $75 \mathrm{~mL}$ square polycarbonate culturing bottles with silicone septa. Two to three replicate bottles were used for each incubation. Acetylene was produced on board by mixing $50 \mathrm{~mL}$ double distilled water with $15 \mathrm{~g}$ calcium carbide and stored in a bladder. Six mL of acetylene were injected into the bottles. The bottles were incubated in a Percival incubator at ambient surface seawater temperatures under soft white fluorescent bulbs at $50 \mu \mathrm{mol}$ quanta $\mathrm{m}^{-2} \mathrm{~s}^{-1}$ light. Zero times were targeted at 11:00 h, 12:00 h, and 13:00 h, local time. At times $0,1 \mathrm{~h}$, and $2 \mathrm{~h}$ after injection, duplicate $0.2-0.3 \mathrm{~mL}$ samples were removed from the headspace of the bottles and injected into a Shimadzu GC-8A gas chromatograph and integrated by a Shimadzu CR8A Chromatopac to measure the ethylene peaks. Standards of $9.1 \mu \mathrm{L} \mathrm{L}^{-1}$ ethylene were used to calibrate the peak heights. Ethylene formed was calculated according to Capone and Montoya (2001) with Bunsen coefficients calculated from surface salinity and temperature according to Breitbarth et al. (2004). Nitrogenase activity was calculated as the average rate of ethylene production per colony $\left(\mathrm{nmol} \mathrm{C}_{2} \mathrm{H}_{4}\right.$ $\mathrm{h}^{-1}$ colony $^{-1}$ ). Acetylene reduction rates for MP09 were provided by D. Capone (pers. comm.). One-way ANOVA Tukey-Kramer tests were used to determine statistically different rates between colony morphologies and among the cruise means $(\alpha=0.05)$. 


\section{Results}

Physical conditions. The North Atlantic upper ocean was stratified with late spring SST ranging from $23^{\circ} \mathrm{C}$ to $25^{\circ} \mathrm{C}$ and MLD ranging from 4 to $37 \mathrm{~m}$ during $\mathrm{EN} 355$ and autumn SST ranging from $25^{\circ} \mathrm{C}$ to $28^{\circ} \mathrm{C}$ and MLD ranging from $70 \mathrm{~m}$ in the northern part of the transect to $26 \mathrm{~m}$ in the south during EN361. During MP09, the summer North Pacific upper ocean was highly stratified with SST ranging from $26.5^{\circ} \mathrm{C}$ to $27.5^{\circ} \mathrm{C}$ and MLD ranging from $25 \mathrm{~m}$ to $58 \mathrm{~m}$. KM0701 cut across the west Pacific warm pool, with early winter deep mixing up to $118 \mathrm{~m}$ in the north, early summer stratification as shallow as $11 \mathrm{~m}$ in the south, and a maximum SST of over $30^{\circ} \mathrm{C}$ just south of the Equator (Fig. 5-2). As the MLD shoaled in the southern end of the west Pacific transect, DIP concentrations decreased (Fig. 5-2).

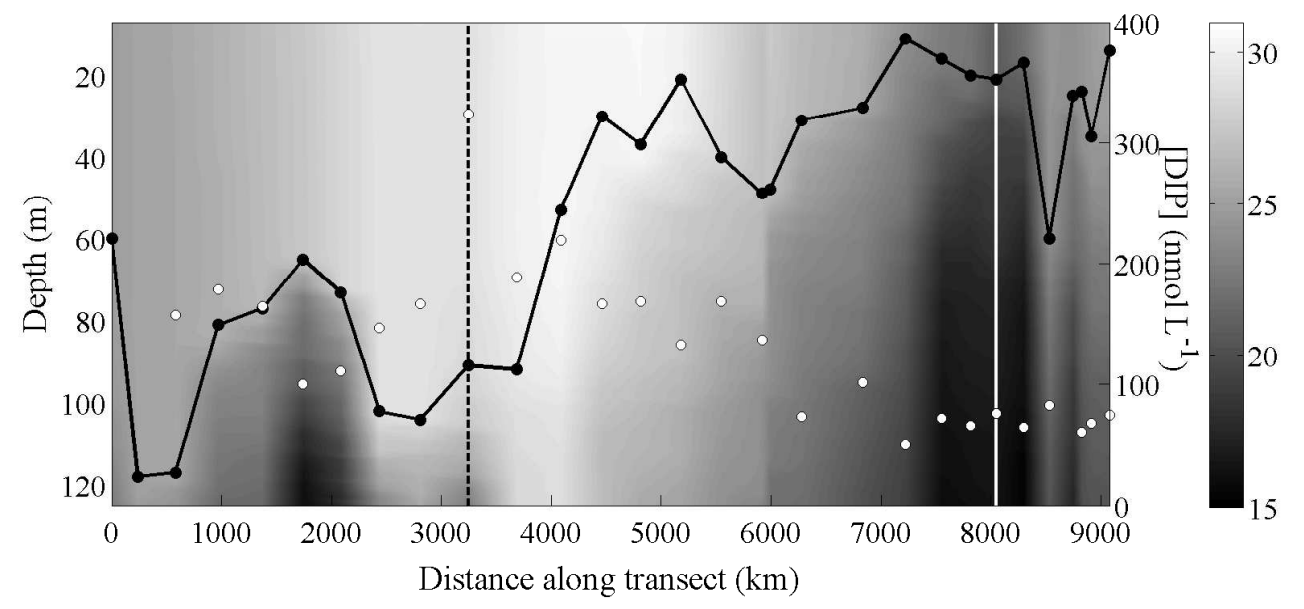

Figure 5-2: Hydrographic conditions of KM0701. The grayscale pseudocolor plot is potential temperature $\left({ }^{\circ} \mathrm{C}\right)$ as a function of depth $(\mathrm{m})$ vs. distance along the transect $(\mathrm{km})$ starting from Hawaii. The connected black circles are mixed layer depth $(\mathrm{m})$ while the white circles indicate DIP concentrations $\left(\mathrm{nmol} \mathrm{L}{ }^{-1}\right)$. The vertical dashed black line represents the Equator and the vertical solid white line denotes a turning point in the transect. 
ELF. North Atlantic samples consistently showed more ELF labeling than Pacific samples. A spatial representation of the maximum ELF labeling at each station is shown along the cruise tracks in Fig. 5-1. During the timeframe of this study, North and South Pacific Trichodesmium field samples did not show high ELF labeling. All samples were rated '+ -' or '- -' , indicating that there was low to no AP activity (Table 5.1; Figs. 5-1 A, B; 5-3 A-H). Labeling of South Pacific Trichodesmium colonies was highest on the shelf near Australia at '+ - ' (Fig. 5-1 A). In contrast, North Atlantic ELF samples ranged from '- -' to ' ++ ' (Table 5.1; Figs. 5-1 C, 5-3 I-P). Labeling of the two colony morphologies was not always at the same level, as seen at Sta. 16, 18, and 20 of MP09 (Table 5.1, Fig. 5-3 A, B) and Sta. 14, 26, and 29 of KM0701 (Table 5.1, Fig. 5-3 G, H). Labeling of colonies at the same station on different days could vary, as seen at MP09 Sta. 17 and 22 (Table 5.1, Fig. 5-3 C, D) and KM0701 Sta. 14 (Table 5.1). Two colonies on the same slide could also differ. For example, Sta. 7 and 8 from EN355 had some highly labeled filaments among unlabeled filaments, giving the slides a rating of '+ -' (Fig. 5-3 J-L). Although Trichodesmium colonies collected were identified by morphology, two to three different filament sizes were observed in over half of the samples (Fig. 5-3 L). Color from the autofluorescence of phycoerythrin in the filaments varied, ranging from the usual orange color (Table 5.1; Fig. 5-3 A, B, E-H) to a dim green or blue (Table 5.1; Fig. 5-3 C, $\mathrm{D}, \mathrm{J})$.

In spite of efforts to wash the colonies for the ELF assay, a variety of microbes were found in association with Trichodesmium colonies including diatoms, heterotrophic bacteria, and the filamentous $\mathrm{N}_{2}$-fixing cyanobacteria Plectonema sp. and Calothrix sp. (Fig. 5-4 A-E). As in Dyhrman et al. (2002), some of these organisms were found to be ELF-labeled even when Trichodesmium was not, indicating AP activity in these other taxa: diatoms (North Pacific MP09-21, North Atlantic EN361-1; Fig. 5-4 A-B), heterotrophic bacteria (over half of the stations on all four cruises; Fig. 5-4 C), and Plectonema sp. (North Pacific MP09-19, North Atlantic EN361-1, 2, 3; Fig. 5-4 D). Calothrix sp. was found in association with Trichodesmium near Hawaii during MP09 and was not 
Table 5.1: Data summary for stations with ELF data. For stations with multiple colony morphologies, data are separated by ' $\%$. NA $=$ not available.

\begin{tabular}{|c|c|c|c|c|c|c|}
\hline Date & Station & Latitute & Longitude & Colony & ELF & Color \\
\hline 01 Jun 01 & EN355-1 & $37^{\circ} 6^{\prime} \mathrm{N}$ & $71^{\circ} 42^{\prime} \mathrm{W}$ & puff/tuft & $--/--$ & $\operatorname{dim} / \operatorname{dim}$ \\
\hline 02 Jun 01 & EN355-2 & $35^{\circ} 52^{\prime} \mathrm{N}$ & $71^{\circ} 43^{\prime} \mathrm{W}$ & puff & +- & $\operatorname{dim}$ \\
\hline 03 Jun 01 & EN355-3 & $33^{\circ} 0^{\prime} \mathrm{N}$ & $71^{\circ} 40^{\prime} \mathrm{W}$ & puff & ++ & $\operatorname{dim}$ \\
\hline 04 Jun 01 & EN355-4 & $31^{\circ} 33^{\prime} \mathrm{N}$ & $71^{\circ} 39^{\prime} \mathrm{W}$ & puff/tuft & $++/+-$ & $\operatorname{dim} / \operatorname{dim}$ \\
\hline 05 Jun 01 & EN355-5 & $30^{\circ} 29^{\prime} \mathrm{N}$ & $70^{\circ} 0.0^{\prime} \mathrm{W}$ & puff/bowtie & NA/NA & NA/NA \\
\hline 07 Jun 01 & EN355-6 & $32^{\circ} 2.4^{\prime} \mathrm{N}$ & $70^{\circ} 19.51^{\prime} \mathrm{W}$ & NA & ++ & dim-normal \\
\hline 09 Jun 01 & EN355-7 & $33^{\circ} 16.67^{\prime} \mathrm{N}$ & $68^{\circ} 59.32^{\prime} \mathrm{W}$ & NA & +- & dim-normal \\
\hline 11 Jun 01 & EN355-8 & $37^{\circ} 27.32^{\prime} \mathrm{N}$ & $69^{\circ} 45.28^{\prime} \mathrm{W}$ & NA & +- & dim-normal \\
\hline 04 Oct 01 & EN361-1 & $36^{\circ} 31.53^{\prime} \mathrm{N}$ & $72^{\circ} 26.78^{\prime} \mathrm{W}$ & puff/tuft & $+-/+-$ & dim-normal/normal \\
\hline 05 Oct 01 & EN361-2 & $33^{\circ} 44.52^{\prime} \mathrm{N}$ & $70^{\circ} 59.79^{\prime} \mathrm{W}$ & puff/tuft & $--/+-$ & dim-norma/dim-normall \\
\hline 05 Oct 01 & EN361-3 & $32^{\circ} 40.89^{\prime} \mathrm{N}$ & $70^{\circ} 9.3^{\prime} \mathrm{W}$ & puff/tuft & $+-/+-$ & dim-normal/dim-normal \\
\hline 06 Oct 01 & EN361-4 & $30^{\circ} 20.35^{\prime} \mathrm{N}$ & $68^{\circ} 22.25^{\prime} \mathrm{W}$ & puff/tuft/bowtie & $+-/+-/+-$ & dim-normal/dim-normal/dim-normal \\
\hline 06 Oct 01 & EN361-5 & $30^{\circ} 6.42^{\prime} \mathrm{N}$ & $67^{\circ} 32.88^{\prime} \mathrm{W}$ & puff/tuft & $+-/+-$ & normal/dim-normal \\
\hline 07 Oct 01 & EN361-6 & $29^{\circ} 10.21^{\prime} \mathrm{N}$ & $64^{\circ} 43.528^{\prime} \mathrm{W}$ & puff/tuft & $+-/+-$ & dim-normal/dim-normal \\
\hline 06 Aug 03 & MP09-13 & $20^{\circ} 29.59^{\prime} \mathrm{N}$ & $157^{\circ} 41.32^{\prime} \mathrm{W}$ & puff/tuft & -- & normal/normal \\
\hline 07 Aug 03 & MP09-14 & $18^{\circ} 30.00^{\prime} \mathrm{N}$ & $157^{\circ} 00.00^{\prime} \mathrm{W}$ & puff & - & normal \\
\hline 10 Aug 03 & MP09-16 & $19^{\circ} 32.49^{\prime} \mathrm{N}$ & $156^{\circ} 55.74^{\prime} \mathrm{W}$ & puff/tuft & $+-/--$ & normal/normal \\
\hline 11 Aug 03 & MP09-16 & $19^{\circ} 30.55^{\prime} \mathrm{N}$ & $156^{\circ} 55.74^{\prime} \mathrm{W}$ & puff/tuft & $+-/--$ & normal/normal \\
\hline 12 Aug 03 & MP09-17 & $19^{\circ} 31.23^{\prime} \mathrm{N}$ & $158^{\circ} 58.75^{\prime} \mathrm{W}$ & puff/tuft & $--/--$ & normal/normal \\
\hline 13 Aug 03 & MP09-17 & $19^{\circ} 30.10^{\prime} \mathrm{N}$ & $158^{\circ} 59.94^{\prime} \mathrm{W}$ & puff & +- & normal \\
\hline 14 Aug 03 & MP09-18 & $20^{\circ} 31.84^{\prime} \mathrm{N}$ & $160^{\circ} 59.81^{\prime} \mathrm{W}$ & puff/tuft & $--/+-$ & $\operatorname{dim} / \operatorname{dim}$ \\
\hline 15 Aug 03 & MP09-19 & $21^{\circ} 00.79^{\prime} \mathrm{N}$ & $158^{\circ} 59.70^{\prime} \mathrm{W}$ & puff/tuft & -- & $\operatorname{dim} /$ normal \\
\hline 16 Aug 03 & MP09-19 & $21^{\circ} 00.18^{\prime} \mathrm{N}$ & $158^{\circ} 59.69^{\prime} \mathrm{W}$ & puff/tuft & $--/--$ & dim/normal \\
\hline 17 Aug 03 & MP09-20 & $20^{\circ} 16.09^{\prime} \mathrm{N}$ & $156^{\circ} 31.72^{\prime} \mathrm{W}$ & puff/tuft & $+-/--$ & normal/normal \\
\hline 18 Aug 03 & MP09-21 & $20^{\circ} 15.61^{\prime} \mathrm{N}$ & $159^{\circ} 11.21^{\prime} \mathrm{W}$ & puff/tuft & $+-/+-$ & normal/dim \\
\hline 19 Aug 03 & MP09-22 & $19^{\circ} 06.67^{\prime} \mathrm{N}$ & $162^{\circ} 13.05^{\prime} \mathrm{W}$ & puff/tuft & $+-/+-$ & normal/dim \\
\hline 20 Aug 03 & MP09-22 & $19^{\circ} 06.29^{\prime} \mathrm{N}$ & $162^{\circ} 13.60^{\prime} \mathrm{W}$ & puff/tuft & $--/--$ & $\operatorname{dim} / \operatorname{dim}$ \\
\hline $21 \mathrm{Aug} 03$ & MP09-23 & $20^{\circ} 30.68^{\prime} \mathrm{N}$ & $161^{\circ} 30.27^{\prime} \mathrm{W}$ & puff/tuft & $--/--$ & normal/dim \\
\hline 07 Jan 07 & KM0701-5 & $12^{\circ} 26.11^{\prime} \mathrm{N}$ & $167^{\circ} 43.75^{\prime} \mathrm{W}$ & puff/tuft & $--/--$ & normal/dim-normal \\
\hline 10 Jan 07 & KM0701-8 & $5^{\circ} 38.86^{\prime} \mathrm{N}$ & $174^{\circ} 32.18^{\prime} \mathrm{W}$ & tuft & - & dim-normal \\
\hline 11 Jan 07 & KM0701-9 & $3^{\circ} 14.40^{\prime} \mathrm{N}$ & $176^{\circ} 53.04^{\prime} \mathrm{W}$ & puff & -- & normal \\
\hline 12 Jan 07 & KM0701-10 & $0^{\circ} 22.02^{\prime} \mathrm{N}$ & $179^{\circ} 38.60^{\prime} \mathrm{W}$ & tuft & -- & normal \\
\hline 15 Jan 07 & KM0701-12 & $4^{\circ} 43.34^{\prime} \mathrm{S}$ & $174^{\circ} 43.85^{\prime} \mathrm{E}$ & puff & -- & normal \\
\hline 16 Jan 07 & KM0701-13 & $7^{\circ} 3.64^{\prime} \mathrm{S}$ & $172^{\circ} 18.98^{\prime} \mathrm{E}$ & tuft & -- & normal \\
\hline 17 Jan 07 & KM0701-14 & $9^{\circ} 15.03^{\prime} \mathrm{S}$ & $169^{\circ} 59.96^{\prime} \mathrm{E}$ & puff/tuft/bowtie & $--/--/--$ & dim-normal/normal/normal \\
\hline 19 Jan 07 & KM0701-14 & $9^{\circ} 15.01^{\prime} \mathrm{S}$ & $170^{\circ} 0.01^{\prime} \mathrm{E}$ & puff/tuft & $--/+-$ & normal/dim-normal \\
\hline 20 Jan 07 & KM0701-15 & $12^{\circ} 34.55^{\prime} \mathrm{S}$ & $169^{\circ} 51.55^{\prime} \mathrm{E}$ & tuft & - & normal \\
\hline 21 Jan 07 & KM0701-16 & $15^{\circ} 53.63^{\prime} \mathrm{S}$ & $169^{\circ} 43.02^{\prime} \mathrm{E}$ & puff & - & normal \\
\hline 22 Jan 07 & KM0701-17 & $19^{\circ} 13.95^{\prime} \mathrm{S}$ & $169^{\circ} 34.63^{\prime} \mathrm{E}$ & puff/tuft & $+-/+-$ & dim-normal/normal \\
\hline 23 Jan 07 & KM0701-19 & $21^{\circ} 37.42^{\prime} \mathrm{S}$ & $168^{\circ} 39.49^{\prime} \mathrm{E}$ & puff/tuft & $--/--$ & normal/normal \\
\hline 26 Jan 07 & KM0701-20 & $25^{\circ} 40.30^{\prime} \mathrm{S}$ & $165^{\circ} 25.03^{\prime} \mathrm{E}$ & NA/puff/tuft & - -/NA/NA & normal/NA/NA \\
\hline 27 Jan 07 & KM0701-21 & $29^{\circ} 2.43^{\prime} \mathrm{S}$ & $164^{\circ} 20.26^{\prime} \mathrm{E}$ & puff/tuft & $--/+-$ & normal/normal \\
\hline 03 Feb 07 & KM0701-26 & $32^{\circ} 25.29^{\prime} \mathrm{S}$ & $159^{\circ} 5.33^{\prime} \mathrm{E}$ & puff/tuft & $--/+-$ & normal/dim-normal \\
\hline 07 Feb 07 & KM0701-28 & $30^{\circ} 15.51^{\prime} \mathrm{S}$ & $157^{\circ} 18.19^{\prime} \mathrm{E}$ & puff/tuft & $+-/+-$ & normal/dim-normal \\
\hline 08 Feb 07 & KM0701-29 & $29^{\circ} 45.56^{\prime} \mathrm{S}$ & $156^{\circ} 37.43^{\prime} \mathrm{E}$ & puff/tuft & $--/+-$ & normal/normal \\
\hline 09 Feb 07 & KM0701-30 & $28^{\circ} 45.68^{\prime} \mathrm{S}$ & $155^{\circ} 22.16^{\prime} \mathrm{E}$ & puff/tuft & $+-/ \mathrm{NA}$ & normal/NA \\
\hline
\end{tabular}




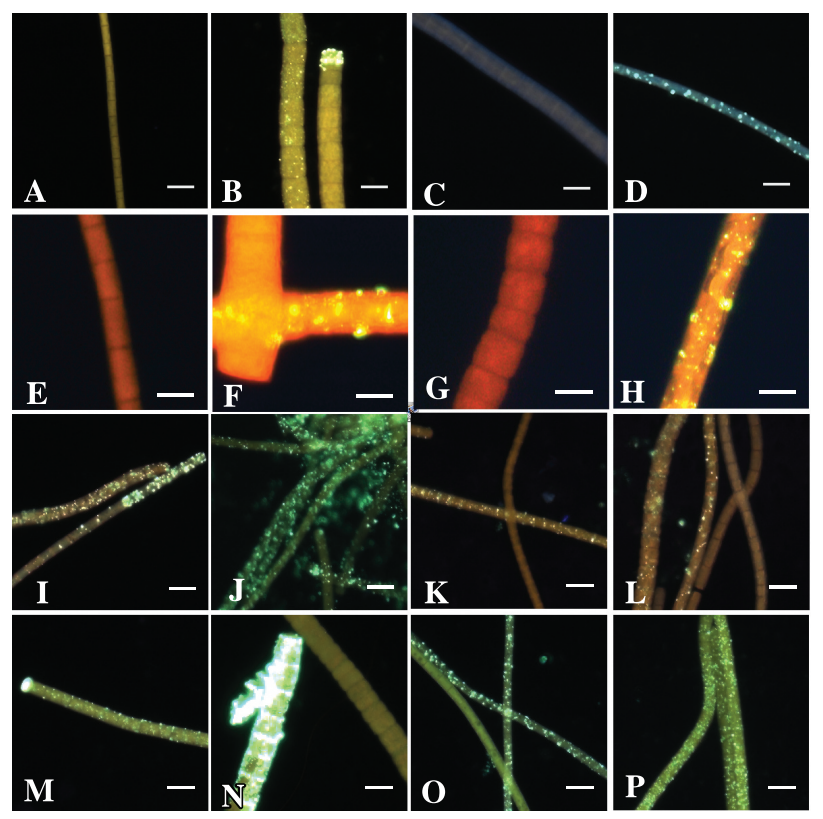

Figure 5-3: Selected digital micrographs of ELF-assayed Trichodesmium from the four cruises: (A-D) MP09 during August 2003, in the North Pacific; (E-H) KM0701 during January and February 2007, across the west Pacific warm pool; and (I-L) EN355 during June 2001, and (M-P) EN361 during October 2001, in the western North Atlantic. White scale bars correspond to $10 \mu \mathrm{m}$. (A) Sta. MP09-16, 10 Aug 03, tuft, '- -'; (B) Sta. MP0916, 10 Aug 03, puff, ‘+ -’; (C) Sta. MP09-22, 20 Aug 03, puff, '- -'; (D) Sta. MP09-22, 19 Aug 03, tuft, ‘+ -'; (E) Sta. KM0701-14, 17 Jan 07, puff, ‘- - '; (F) Sta. KM0701-17, 22 Jan 07, puff, ‘+ -'; (G)Sta. KM0701-26, 03 Feb 07, puff, '- - '; (H) Sta. KM0701-26, 03 Feb 07, tuft, ‘+ -’; (I) Sta. 6-6, 07 Jun 01, ‘++ '; (J) Sta. 6-7, 09 Jun 01, ‘+ -’; (K) Sta. 6-7, 09 Jun 01, ‘+ -’; (L) Sta. 6-8, 11 Jun 01, ‘+ -’; (M) Sta. 10-1, 04 Oct 01, puff, ‘+ -’; (N) Sta. 10-2, 05 Oct 01, tuft, '++'; (O) Sta. 10-3, 05 Oct 01, puff, '+ -'; (P) Sta. 10-5, 06 Oct 01 , puff, '+ -'. 

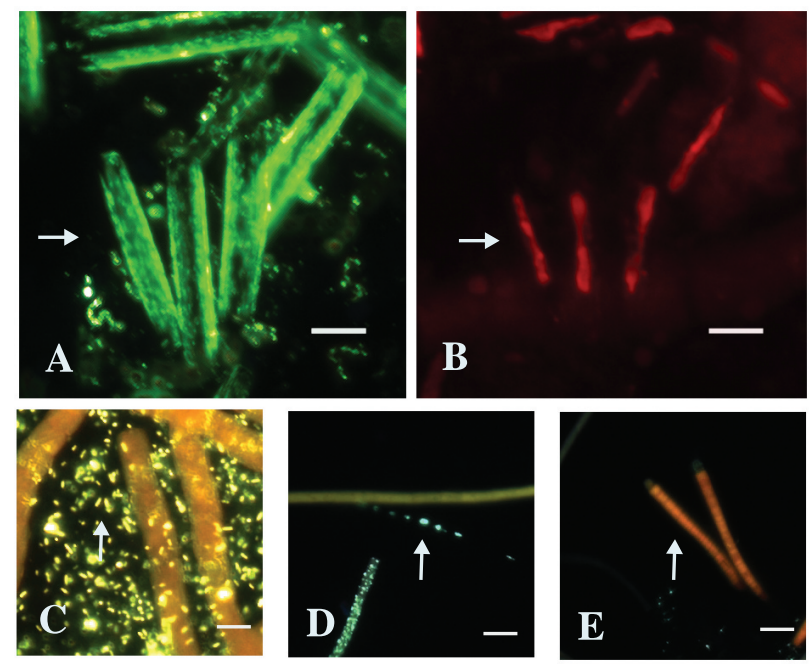

Figure 5-4: Organisms associated with Trichodesmium colonies. White arrows indicate the organisms and white scale bars correspond to $10 \mu \mathrm{m}$. (A) ELFlabeled pennate diatoms (Sta. MP0917); (B) the same pennate diatoms in (A) viewed under a chlorophyll filter; (C) ELF-labeled heterotrophic bacteria (Sta. MP09-16, 11 Aug 03, puff); (D) ELF-labeled cyanobacterium Plectonema sp. (Sta. MP09-19, 15 Aug 03, puff); (E) $\mathrm{N}_{2}$-fixing cyanobacterium Calothrix sp. (Sta. MP09-23, 21 Aug 03, tuft).

ELF-labeled (Sta. 13, 17, 18, 19, 21, 23; Fig. 5-4E).

DIP and ELF. ELF labeling varied with ocean basin and corresponded negatively with DIP concentrations. North Atlantic stations had significantly lower DIP concentrations than the North and South Pacific stations in a 2-way ANOVA ( $p=0.0001)$ (Table 5.2). DIP concentrations ranged from below detection level (BDL) to $25 \mathrm{nmol} \mathrm{L}{ }^{-1}$ in the North Atlantic, 30 to $320 \mathrm{nmol} \mathrm{L}^{-1}$ in the North Pacific, and 50 to $220 \mathrm{nmol} \mathrm{L}^{-1}$ in the South Pacific. Stacked histograms of DIP concentrations were plotted for each ELF label rating (Fig. 5-5). Trichodesmium colonies with little or no ELF labeling were found in stations from all cruises, spanning the full range of DIP concentrations (Fig. 5-5 A). Samples with moderate levels of labeling spanned a moderate range of DIP: BDL - $169 \mathrm{nmol}$ $\mathrm{L}^{-1}$ (Fig. 5-5 B). High levels of labeling were found only in samples from North Atlantic stations with DIP concentrations less than or equal to $40 \mathrm{nmol} \mathrm{L}^{-1}$ (Fig. 5-5 C). In a 2way ANOVA, DIP concentrations for stations with an ELF rating of '- -' were greater than DIP concentrations for stations with an ELF rating of ' + -' or ' ++ ' , significant at the 94\% confidence level ( $p=0.0554)$ (Table 5.2). 
Table 5.2: 2-way ANOVA on DIP concentrations among ELF ratings and ocean basins

\begin{tabular}{lrrl}
\hline Factor & $F$ & $p$ & Groupings \\
\hline ELF rating & 3.04 & 0.0554 & '++' and '+ -'; '- -' \\
Ocean basin & 11.55 & 0.0001 & North Atlantic; North and South Pacific \\
\hline
\end{tabular}
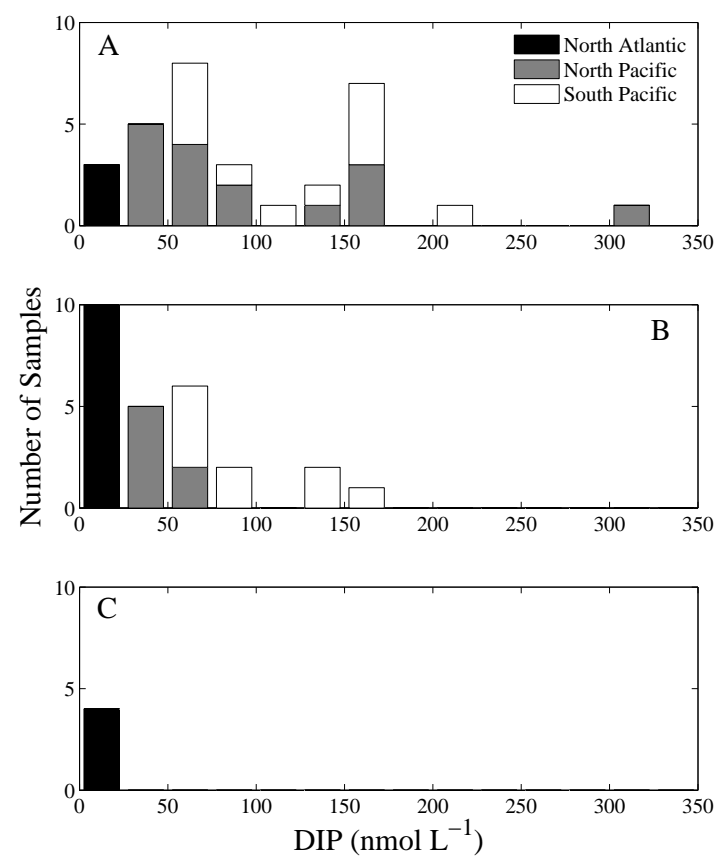

Figure 5-5: Histogram of binned surface DIP concentrations (nmol L ${ }^{-1}$ ) separated by ELF label ratings: (A) '- -,' (B) ‘+ -,' (C) ‘++.' Colors represent ocean basin: North Atlantic (black), North Pacific (gray), and South Pacific (white). Bin width is $25 \mathrm{nmol}$ $\mathrm{L}^{-1}$.

Nitrogenase activity. Nitrogenase activity varied significantly between the basins (Table 5.3, Fig. 5-6). In the P-stressed western North Atlantic, mean nitrogenase activities measured during EN355 and EN361 were significantly higher than rates in both the North and South Pacific $\left(p=5.35 \times 10^{-8}\right): 0.80 \pm 0.22$ and $0.79 \pm 0.14 \mathrm{nmol} \mathrm{C}_{2} \mathrm{H}_{4} \mathrm{~h}^{-1}$ colony $^{-1}$ compared to $0.26 \pm 0.03 \mathrm{nmol} \mathrm{C}_{2} \mathrm{H}_{4} \mathrm{~h}^{-1}$ colony $^{-1}$ (D. Capone pers. comm.) and $0.09 \pm$ $0.02 \mathrm{nmol} \mathrm{C}_{2} \mathrm{H}_{4} \mathrm{~h}^{-1}$ colony $^{-1}$, respectively (Fig. 5-6). For stations with nitrogenase ac- 
tivity measurements for both puff and tuft colonies, rates were generally greater for tuft colonies than puff colonies although the rates were not significantly different between colony morphs according to one-way ANOVA Tukey-Kramer tests ( $p \geq 0.1355)$. There were two exceptions to this pattern. At Sta. KM0701-14, puff nitrogenase activity was greater than that of tufts but not significantly $(p=0.2379)$, and at Sta. KM0701-30 near Australia, the rate for tuft colonies was significantly greater than that of puff colonies $(p=0.0022)$. 


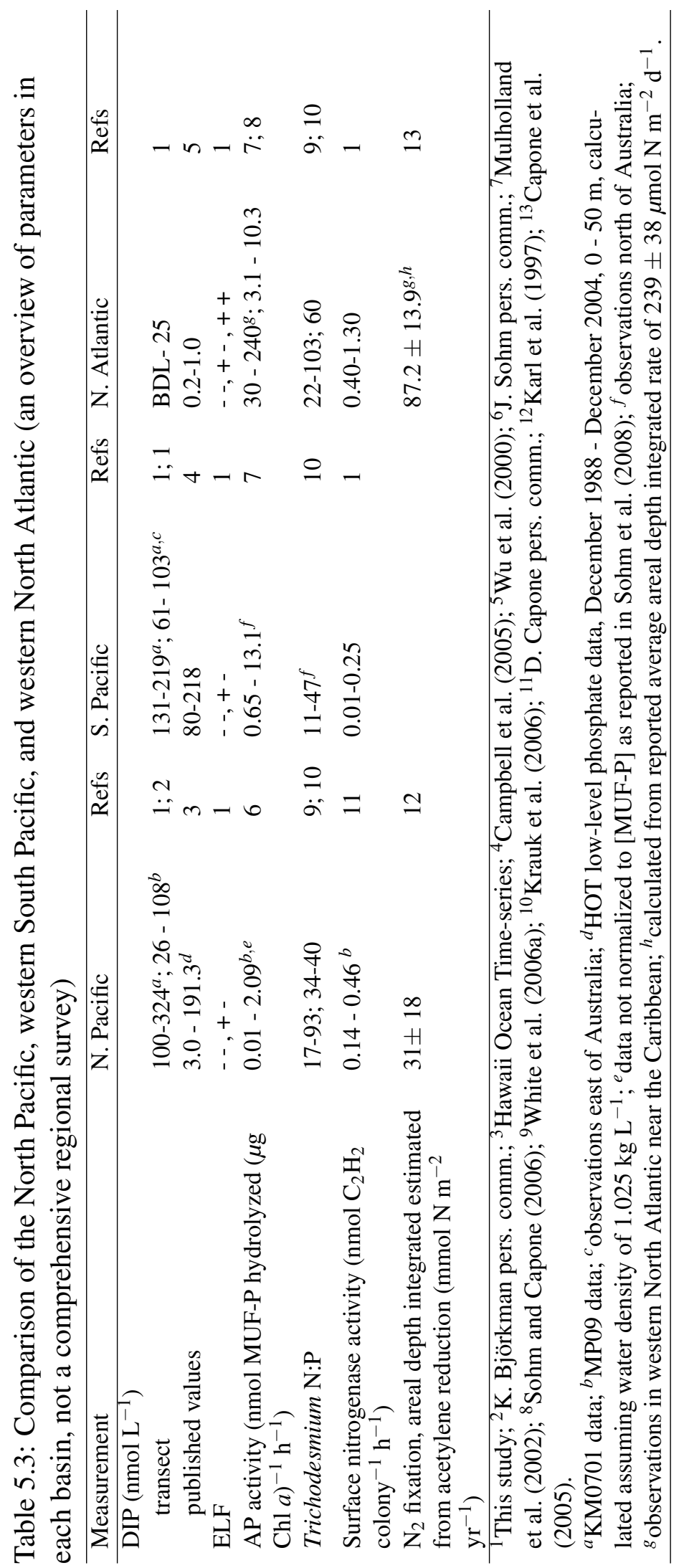




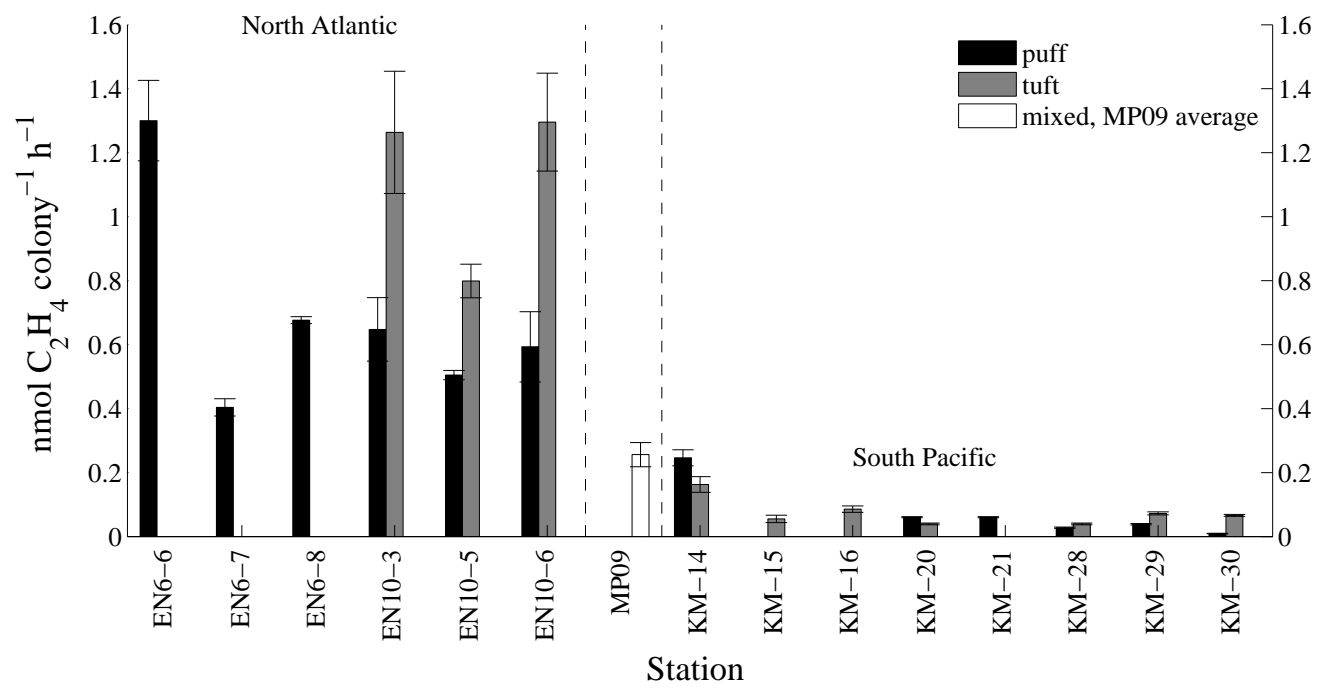

Figure 5-6: Bar plot of nitrogenase activity (nmol $\mathrm{C}_{2} \mathrm{H}_{4}$ produced colony ${ }^{-1} \mathrm{~h}^{-1}$ ) for each station. Error bars show standard error of the mean. Data is separated by puffs (black), tufts (gray), or mixed puffs and tufts (white). The North Pacific is represented by the average rates for MP09 Stas. 14-22 (D. Capone, pers. comm.). Ocean basins are separated by vertical dotted lines.

\section{Discussion}

Trichodesmium is a source of new $\mathrm{N}$ in tropical and subtropical oceans around the world, but the factors limiting $\mathrm{N}_{2}$ fixation in Trichodesmium are not completely understood. The South Pacific in particular is an undersampled region (Campbell et al., 2005). Our study investigated $\mathrm{P}$ stress and $\mathrm{N}_{2}$ fixation in Trichodesmium in three major ocean regions: the western North Atlantic, the North Pacific, and the western South Pacific. Our results show patterns of both higher $\mathrm{P}$ stress and higher $\mathrm{N}_{2}$ fixation rates in the western North Atlantic than in the Pacific.

During the 2001 western North Atlantic cruises, colonies of Trichodesmium showed more frequent and more complete ELF labeling than the Pacific samples in this study. This difference in ELF labeling is a qualitative indication of higher AP activity in the North Atlantic. The samples near Australia also showed more labeling than other regions 
of the South Pacific. In this region the MLD was shallower and the DIP concentrations were lower. Trichodesmium thrives in warm, stratified waters, and blooms are found in oligotrophic waters where the sea surface temperature (SST) $\geq 25^{\circ} \mathrm{C}$ (Karl et al., 2002). However, supply of P to surface waters can be cut off by warming and stratification of surface layers. ELF is not quantitative, so finding a direct parametric correlation with DIP concentrations is not possible. However, by examining the range of DIP concentrations associated with the different levels of ELF label, it is apparent that higher labeling is associated with lower DIP concentrations. This is in contrast to Webb et al. (2007), where they found no significant relationship between ELF labeling and DIP during a transect in the western Equatorial to western South Atlantic. In our study, we had more data points of ELF and a broader range of DIP than (Webb et al., 2007) (5-320 $\mathrm{nmol} \mathrm{L}^{-1}$ compared to 10-120 nmol L ${ }^{-1}$ ), and this breadth of data may have enabled us to find a significant relationship between DIP and ELF labeling. Also in contrast to (Webb et al., 2007), we did not see consistent differences in labeling between puff and tuft colonies. In a study of the North and South Atlantic subtropical gyres, surface water column AP activity was higher and DIP was lower in the North than the South Atlantic (Mather et al., 2008). AP activity in the NPSG measured during cruise MP09 was 4.4 times lower than AP activity in the North Atlantic (Sohm et al., 2008) which is consistent with our ELF-based observations. The difference in AP activity observed by ELF labeling and hydrolytic rates among these regions suggests that the Atlantic populations were generally more P-stressed than those in the Pacific.

In addition to AP activity, indicators used to determine $\mathrm{P}$ stress or potential limitation include DIP concentrations, maximum uptake rate of $\mathrm{PO}_{4}^{3-}\left(\mathrm{V}_{\max }\right)$, cellular $\mathrm{N}: \mathrm{P}$ ratios, and turnover times of DIP. Results from these metrics used in the tropical and subtropical Atlantic and Pacific Oceans are consistent with our observations of AP activity. The critical concentration of DIP for Trichodesmium growth has been determined to be 9 nmol L ${ }^{-1}$ (Moutin et al., 2005). While there is some overlap, Atlantic DIP concentrations are typically lower than this critical value while Pacific concentrations are typically higher 
(Table 5.3) (Wu et al., 2000). The $\mathrm{V}_{\text {max }}$ of $\mathrm{PO}_{4}^{3-}$ uptake by Trichodesmium colonies measured during a cruise in the North Atlantic was four times more than that of the North Pacific during cruise MP09 (Sohm et al., 2008). Concentrations of DIP in the Sargasso Sea region of the Atlantic are 1-2 orders of magnitude lower than those in the subtropical North Pacific (Wu et al., 2000). Molar ratios of cellular N:P may indicate nutrient limitation and/or storage. The reference Redfield ratio of $\mathrm{N}: \mathrm{P}$ is 16 . The $\mathrm{P}$ limitation of growth in Trichodesmium begins at N:P of 40-50 (Kustka et al., 2003), and in P-restricted cultures, N:P can reach $\geq 90$ (White et al., 2006a). Trichodesmium N:P ratios averaged $40.1 \pm 2.53$ during the North Pacific MP09 cruise while they ranged from 60 in the west to 30 in the east in the subtropical North Atlantic in 2000, indicating more P limitation in the western North Atlantic (Krauk et al., 2006). Turnover time is calculated as the inventory of a nutrient divided by its uptake rate and can be considered as the period it would take to deplete a nutrient if input was halted (Ammerman et al., 2003). Short turnover times of DIP $\left(\tau_{D I P}\right)$ result from low inventory and/or high uptake rates and can indicate low DIP availability. At the Climax station in the North Pacific, $\tau_{D I P}$ was $48 \mathrm{~h}$ in the summer (Björkman et al., 2000). In contrast, Sargasso Sea $\tau_{D I P}$ was measued to be around 9 h (Cotner et al., 1997). Turnover times of DIP in the South Pacific near New Caledonia varied widely and decreased from $468 \mathrm{~h}$ in the winter to $4 \mathrm{~h}$ in the early fall, indicating $\mathrm{P}$ depletion after the stratification of the water column in the summer (Van Den Broeck et al., 2004). These $\mathrm{P}$ stress indicators are consistent with higher potential for $\mathrm{P}$ limitation in the subtropical western North Atlantic than the subtropical North and South Pacific with a region of summer P limitation in the South Pacific near New Caledonia.

Colonies used in the ELF assay were chosen in an attempt to represent the community, but a number of issues arise because of the small sample size. Field populations of Trichodesmium have a range of characteristics including nutrient history, species, and degree of senescence which might not be reflected in two colonies per morphology per station. ELF labeling varies within a sample, a colony, or even along a filament. While colonies of the same morphology were targeted, filaments observed on the ELF slides 
were from a variety of Trichodesmium species based on cell width and length. Samples also displayed a range of phycobiliprotein autofluorescence. The dim filaments observed may be due to senescent colonies, bleaching, or fading. In some cases, labeling on a slide was higher on dim filaments than normal filaments, which may indicate elevated AP activity in senescent colonies or in heterotrophic bacteria associated with these colonies in some cases. While the colonies picked for the ELF assay may not capture the diversity of field populations, they do give useful snapshots of the state of the community.

A variety of organisms including bacteria, fungi, diatoms, dinoflagellates, chrysophytes, ciliates, amoebae, hydroids, and crustacean larvae can be enriched by $2-5$ orders of magnitude in association with Trichodesmium colonies (Sheridan et al., 2002). Hydrolysis rates measured on Trichodesmium colonies may reflect the activity of the whole community rather than Trichodesmium-specific activity (Dyhrman et al., 2002; Webb et al., 2007)). The activity of AP from associated organisms such as pennate diatoms, heterotrophic bacteria, and Plectonema may result in an indirect source of DIP that can be utilized by Trichodesmium. The presence of AP in these microorganisms may also result in the overestimation of Trichodesmium AP activity measured through bulk assays. With ELF, AP activity can be visually localized to specific cells.

In addition to AP measurements, $\mathrm{N}_{2}$ fixation rates attributed to Trichodesmium may be influenced by the diazotrophs tentatively identified as Plectonema sp. and Calothrix sp. found in close association with Trichodesmium colonies. Both of these cyanobacteria occupy a wide variety of habitats, including marine. The long, thin filaments of nonheterocystous Plectonema are found in Trichodesmium colonies around the world (Siddiqui et al., 1992). Calothrix is identified by its terminal heterocyst and tapering trichome (Waterbury, 2006), and is found in the upper intertidal zone (Whitton and Potts, 1982) and as an epiphyte of the pelagic diatom Chaetoceros (Foster and Zehr, 2006). While Plectonema is known to fix $\mathrm{N}_{2}$ only in lowered oxygen tension (Rippka et al., 1979), both Plectonema and Calothrix may be contributing to $\mathrm{N}_{2}$ fixation rates measured from Trichodesmium colonies. 
During the four cruises analyzed in this study, Trichodesmium colony-based $\mathrm{N}_{2}$ fixation rates were significantly higher in the subtropical North Atlantic than in the tropical and sub-tropical North and South Pacific. North Atlantic values averaged $0.80 \pm 0.12$ nmol $\mathrm{C}_{2} \mathrm{H}_{4} \mathrm{~h}^{-1}$ colony $^{-1}$ while Pacific values averaged $0.13 \pm 0.02 \mathrm{nmol} \mathrm{C}_{2} \mathrm{H}_{4} \mathrm{~h}^{-1}$ colony $^{-1}$. As in Webb et al. (2007), tuft colonies generally had higher per colony rates than puffs, however the difference between colony morphologies were not significant in our study with one exception. For comparison, literature values of $\mathrm{N}_{2}$ fixation rates measured by acetylene reduction averaged $87 \pm 14 \mathrm{mmol} \mathrm{N} \mathrm{m}^{-2} \mathrm{yr}^{-1}$ in the western North Atlantic from 1994-2003 $\left(1.6 \times 10^{12} \mathrm{~mol} \mathrm{~N} \mathrm{yr}^{-1}\right.$ over $17.8 \times 10^{6} \mathrm{~km}^{2}$; (Capone et al., 2005)), while the North Pacific averaged $31 \pm 18 \mathrm{mmol} \mathrm{N} \mathrm{m}^{-2} \mathrm{yr}^{-1}$ from 1990-1992 (Karl et al., 1997). Biogeochemical estimates of the annual input of new $\mathrm{N}$ by $\mathrm{N}_{2}$ fixation were $2.0 \times 10^{12} \mathrm{~mol} \mathrm{~N} \mathrm{yr}^{-1}$, or $72 \mathrm{mmol} \mathrm{N} \mathrm{m}^{-2} \mathrm{yr}^{-1}$ in the subtropical North Atlantic (Gruber and Sarmiento, 1997) and $4 \pm 1 \times 10^{12} \mathrm{~mol} \mathrm{~N} \mathrm{yr}^{-1}$, or $39 \pm 9 \mathrm{mmol} \mathrm{N} \mathrm{m}^{-2} \mathrm{yr}^{-1}$ in the tropical and subtropical Pacific (Deutsch et al., 2001). In a global biogeochemical elemental cycling ocean model, $\mathrm{N}_{2}$ fixation rates averaged $18 \mathrm{mmol} \mathrm{N} \mathrm{m}^{-2} \mathrm{yr}^{-1}$ in the tropical and subtropical Atlanic and $11 \mathrm{mmol} \mathrm{N} \mathrm{m}^{-2} \mathrm{yr}^{-1}$ in the tropical and subtropical Pacific (Moore and Doney, 2007). Our nitrogenase activity measurements are in congruence with the pattern found in these estimates; $\mathrm{N}_{2}$ fixation rates are higher in the tropical and subtropical North Atlantic than in the tropical and subtropical Pacific.

The difference in $\mathrm{N}_{2}$ fixation rates between the Atlantic and Pacific Oceans may be due to disparate nutrient limitation regimes. $\mathrm{N}_{2}$ fixation in the western North Atlantic is more P-limited than Fe-limited (Wu et al., 2000; Sañudo-Wilhelmy et al., 2001; Moore et al., 2004). When Trichodesmium cells have met their Fe cell quota, $\mathrm{N}_{2}$ fixation correlates with cellular P quotas (Sañudo-Wilhelmy et al., 2001). In the western Equatorial and South Atlantic, $\mathrm{N}_{2}$ fixation in shipboard incubations was higher when P stress was relieved by the addition of DIP than in control or Fe addition incubations (Webb et al., 2007). Trichodesmium AP activity is higher in the North Atlantic than in the Pacific Ocean (Table 5.3) (Mulholland et al., 2002; Sohm and Capone, 2006; Sohm et al., 2008), 
and this difference is reflected by the visualization of AP activity through ELF (Table 5.3, Figs. 5-1, 5-3).

In contrast, $\mathrm{N}_{2}$ fixation in the North Pacific may be limited by factors other than P. The North Pacific receives less Fe-rich dust input than the North Atlantic (Fung et al., 2000), and Fe is necessary for Trichodesmium growth and the enzyme nitrogenase used in $\mathrm{N}_{2}$ fixation. In the South Pacific, regions near New Caledonia and Australia receive Fe from continental margins and Australian dust (Moore and Braucher, 2008). Fe stimulates the growth of diazotrophs, drawing down P (Karl, 2002; Mather et al., 2008), so Fe-rich areas such as the western North Atlantic and western South Pacific near Australia are more likely to be P-limited. In a coupled biogeochemistry ecosystem circulation model, diazotrophs were limited by $\mathrm{P}$ in the Atlantic and by Fe in the Pacific, and increasing Fe inputs in the model increased $\mathrm{N}_{2}$ fixation in the tropics and subtropics (Moore et al., 2004). These data suggest that $\mathrm{N}_{2}$ fixation rates of Trichodesmium in the western North Atlantic and in the South Pacific near Australia are limited by P while the Pacific subtropical gyres may be limited by Fe or co-limited by Fe and P.

While P limitation may be alleviated by the use of DOP by Atlantic Trichodesmium, Fe limitation in Pacific populations cannot be alleviated by the use of an alternative pool (Webb et al., 2007; Mather et al., 2008). In a comparison of the North and South Atlantic, DIP released from AP activity in the P-stressed North Atlantic supported up to $30 \%$ of primary production and carbon fixation rates there were comparable to rates in the $\mathrm{P}$ replete South Atlantic (Mather et al., 2008). Fe may be the proximal limiting nutrient according to Leibig's law while P may be the ultimate limiting nutrient, controlling productivity over long timescales (Tyrrell, 1999). Additional assessments of Trichodesmium $\mathrm{P}$ and Fe physiology in open ocean gyres are necessary to elucidate nutrient controls on $\mathrm{N}_{2}$ fixation, but our study supports previous observations that the North Atlantic is P-limited while the Pacific is likely to be Fe-limited. This study underscores the contrasting importance of $\mathrm{P}$ (and likely Fe) to the activity of the key diazotroph Trichodesmium in different ocean basins. 
Acknowledgements. We thank J. Sohm and K. Björkman for MP09 data and comments on drafts for this manuscript, J. Waterbury for assistance at sea for EN355 and EN361, D. Masten and S. Becker for dissolved inorganic phosphorus (DIP) analysis for EN355 and EN361, and J. Jennings for DIP analysis for KM0701. Special thanks to V. Starczyk for assistance in the statistical analysis of the enzyme-labeled fluorescence (ELF) data. We wish to acknowledge two anonymous reviewers whose contributions improved this manuscript. We also express our gratitude to the captain, crews, and named chief scientists of the following cruises: Z. Johnson and E. Zinser of the R/V Kilo Moana cruise KM0701, D. Capone of the R/V Revelle cruise MP09 (as well as our collaborators involved in the Marine Nitrogen Transformation (MANTRA) project), and H. Sosik and R. Olson of the R/V Endeavor cruises EN355 and EN361. This work was supported from the National Science Foundation (NSF) Biocomplexity Program Grant (OCE-0323332); the NSF Biological Oceanography Program (OCE-0220945); NSF grants to EAW (OCEBO-0220945 and OCE-CO-0623499); Woods Hole Oceanographic Institution (WHOI) fellowship to EAW, National Defense Science and Engineering Graduate (NDSEG) fellowship to PDC; and the Center for Microbial Oceanography Research and Education (C-MORE), an NSF Science and Technology Center (EF-0424599). 


\section{CHAPTER 6}

\section{Concluding remarks}

\section{Thesis summary}

My thesis investigated the potential for niche differentiation in the filamentous diazotroph Trichodesmium. In Chapt. 2, I found that there were two clades of Trichodesmium among our cultured strains. Clade I was comprised of Trichodesmium erythraeum and Trichodesmium contortum, and clade II consisted of Trichodesmium thiebautii, Trichodesmium tenue, Trichodesmium hildebrandtii, and Trichodesmium pelagicum. There was a surprising amount of diversity in cell morphology within each clade. The makeup of accessory pigments (phycobiliproteins) within each clade was consistent, indicating that Trichodesmium niches may be partially defined by light quality. Using sequences of hetR from Chapt. 2, I developed a real-time quantitative polymerase chain reaction ( $\mathrm{qPCR}$ ) protocol that was capable of distinguishing between the two clades in mixture (Chapt. 3). The qPCR protocol was applied to depth profiles across transects of the Equatorial Atlantic Ocean, the west Pacific warm pool, and the South Pacific Ocean (Chapt. 4). I found that concentrations of clade II were generally higher than those of clade I, and both clades reached higher concentration in the Atlantic than in the Pacific. Concentrations of the two clades were correlated in the Pacific Ocean, indicating that similar factors were controlling their distribution. However, the two clades were not correlated in the Altantic Ocean, indicating that different factors were limiting the distribution of each clade. Clade I had deeper and 
cooler ranges than clade II, suggesting that light and/or temperature may define the niches of different types of Trichodesmium. Concentrations of Trichodesmium did not correlate with dissolved inorganic phosphorus (DIP) or iron (Fe) concentrations, but ratios of the clades (clade II:clade I) were consistently high in stations with low dissolved DIP, notably the Atlantic. Differential competition for resources may allow the two clades of Trichodesmium to coexist, but more studies are required to understand how light and nutrients limit the growth of different species of Trichodesmium. In Chapt. 5, I investigated phosphorus (P) stress and $\mathrm{N}_{2}$ fixation in populations in the western North Atlantic, North Pacific, and the west Pacific warm pool (Hynes et al., 2009). Atlantic populations had more $\mathrm{P}$ stress and higher $\mathrm{N}_{2}$ fixation rates than Pacific populations, indicating that factors other than $\mathrm{P}$ were limiting Trichodesmium $\mathrm{N}_{2}$ fixation.

\section{Evolution of Trichodesmium}

There is diversity within the genus Trichodesmium, but where does this diversity come from? There are two major clades of Trichodesmium, even if one includes the field samples of T. contortum, T. tenue, and Trichodesmium aureum not represented in culture (Janson et al., 1999; Lundgren et al., 2005). These two clades are circumtropical, existing in tropical and subtropical waters around the globe. This implies that there was a divergence early in the evolution of Trichodesmium before the spread of this genus around the globe. Within each clade, species are similar genetically but have vastly different phenotypes (Chapt. 2). Consider clade I; T. erythraeum has mostly square cells about $7 \mu \mathrm{m}$ wide while T. contortum has disc-shaped cells about $25 \mu \mathrm{m}$ wide and $3 \mu \mathrm{m}$ long. Given the high genetic similarity of these two species based on 16S, ITS, and hetR, their disparate phenotypes may be due to differences in gene regulation or post-translational controls.

Nitrogen fixation is probably the most important ecological role for Trichodesmium. Based on nifH sequences, Trichodesmium diverged from other diazotrophs early in evolution (Capone et al., 1997). While Trichodesmium diverged from other groups, nifH 
sequences among species of Trichodesmium are highly similar (Lundgren et al., 2001), conserving the structure of the $\mathrm{N}_{2}$-fixing enzyme nitrogenase. Regulation of $\mathrm{N}_{2}$ fixation in Trichodesmium may be carried out in part by hetR (El-Shehawy et al., 2003). Purifying selection on hetR was stronger for T. erythraeum than for other lineages of Trichodesmium (Mes and Stal, 2005). While the structure of nitrogenase in Trichodesmium is conserved, its regulation might vary between clades.

The copepod Macrosetella gracilis is one of the few known grazers of Trichodesmium (O’Neil and Roman, 1994; O'Neil, 1998), but grazing pressure is considered to be low (Carpenter et al., 1987; Eberl and Carpenter, 2007). T. thiebautii is known to produce a neurotoxin, which has not been reported in T. erythraeum, and copepods graze preferentially on T. erythraeum (O'Neil and Roman, 1994). Differential grazing pressure may have led to the production of neurotoxins in clade II, but not clade I.

In Chapt. 4, I found that clade II was numerically more dominant in both the Pacific and Atlantic Oceans, but clade I had a deeper range than clade II in field populations. The dominance of clade II and the wider physical range of clade I are reminiscent of the classic intertidal study of barnacles by Connell (1961). Cthamalus stellatus was out-competed by Semibalanus balanoides in lower reaches of the intertidal zone, but $C$. stellatus was better able to withstand dessication in the upper intertidal zone than S. balanoides. Two characteristics that could affect the ability of Trichodesmium spp. to thrive at depth include pigment composition and gas vesicle strength. In Chapt 2, I found that clade I strains had high phycoerythrobilin (PEB), which absorbs light with wavelengths of about $545 \mathrm{~nm}$ (green) and clade II strains had high phycourobilin (PUB), which absorbs at $495 \mathrm{~nm}$ (blue-green) (Chapt. 2). Blue wavelengths penetrate deeper in the water column than green wavelengths, so it would seem that PUB, and hence clade II, would be better adapted for life at depth. Unexpectedly, we found clade I at depth. T. erythraeum cultures have been shown to alter pigment ratios in response to light quality and quantity (Bell and Fu, 2005). Deep clade I populations might have higher PUB:PEB ratios than what we measured in cullture. Subramaniam et al. (1999) has suggested that high PUB 
in T. thiebautii field populations at midday might be a mechanism to reduce photooxidative stress. The relationship between pigmentation of Trichodesmium and light utilization is a subject of further study. Gas vesicle strength is also variable among species of Trichodesmium. Gas vesicles in Trichodesmium spp. are much stronger than those in other cyanobacteria, a characteristic which may be the result of natural selection for the ability to withstand pressure and maintain buoyancy at depth (Walsby, 1992). Critical pressures for gas vesicles in T. thiebautii (3.7 MPa) and T. contortum (3.4 MPa) were higher than that of T. erythraeum (1.2 MPa) (Walsby, 1992). This variation in gas vesicle strength does not fall along phylogenetic lines, so either there is variability within clade I, or due to the overlapping physical characteristics of T. erythraeum and T. thiebautii, they were misidentified. It is difficult to resolve species identification in the absence of genetic data. The interpretation of pigment and gas vesicle data is unclear, but inter-clade competition may have resulted in the selection for characteristics which would enable clade I species to survive at greater depths than clade II.

\section{Future studies}

Understanding the factors that influence the distributions of Trichodesmium spp. enables us to predict the contributions they make to the global marine nitrogen $(\mathrm{N})$ and carbon (C) cycles. Current ecosystem models incorporating $\mathrm{N}_{2}$ fixation are often based on Trichodesmium, particularly T. erythraeum (clade I). However, the dominant phylotype in the open ocean is clade II. If these two types of Trichodesmium are significantly different in their niche spaces, the models may not accurately represent the ecologically important species of Trichodesmium. Future studies should aim to define the differences among species of Trichodesmium and identify the mechanisms which enable them to coexist. Physiological experiments should be extended to include T. contortum and representatives of clade II. The responses of the two clades to nutrient limitation is under investigation: P. D. Chappell has been investigating Fe (Chappell, 2009), and E. D. Orchard has been investigating P. Previous experiments that focused on T. erythraeum should be extended 
to include more species: chromatic adaptation in response to light quality (Bell and $\mathrm{Fu}$, 2005), growth and $\mathrm{N}_{2}$ fixation rates in response to temperature (Breitbarth et al., 2007), and the possible effects of increasing atmospheric $\mathrm{CO}_{2}$ (Hutchins et al., 2007). Ecosystem models should incorporate the diversity of Trichodesmium and test whether the differences between the two clades are ecologically important.

To be able to define the niche space for each species of Trichodesmium and understand the mechanisms which enable them to coexist, culturing efforts must be extended to encompass the full diversity of Trichodesmium spp. The I-80 melting type of clade I found in Chapt. 4 had a deeper range than the rest of clade I. It also resembled sequences of Trichodesmium field samples found at $75 \mathrm{~m}$ (Janson et al., 1999). We should target deep Trichodesmium for culturing. Higher diversity within the culture collection leads to the possibility of developing a species-specific method for enumerating Trichodesmium.

My thesis has been a step toward investigating Trichodesmium at a higher resolution, both spatially and taxonomically. While we are far from resolving Hutchinson's "plankton paradox," we are beginning to understand that there is important diversity within the genus Trichodesmium. 


\section{Bibliography}

Ahlgren, N. A., G. Rocap, and S. W. Chisholm, 2006, Measurement of Prochlorococcus ecotypes using real-time polymerase chain reaction reveals different abundances of genotypes with similar light physiologies. Environ. Microbiol, , 8:441-454, doi: 10.1111/j.1462-2920.2005.00910.x [73, 86]

Ammerman, J. W., R. R. Hood, D. A. Case, and J. B. Cotner, 2003, Phosphorus deficiency in the Atlantic: an emerging paradigm in oceanography. EOS, 84:165-170 [121, 137]

Anagnostidis, K. and J. Komárek, 1988, Modern approach to the classification system of Cyanophytes 3-Oscilatoriales. Arch. Hydrobiol. Suppl., 80:327-472 [36, 37, 38, 67]

Bailey, B. A., S. C. Doney, and I. D. Lima, 2004, Quantifying the effects of dynamical noise on the predictability of a simple ecosystem model. Environmetrics, 15:337-355, doi:10.1002/env.645 [29]

Bell, P. R. F. and F. X. Fu, 2005, Effect of light on growth, pigmentation and $\mathrm{N}_{2}$ fixation of cultured Trichodesmium sp. from the Great Barrier Reef lagoon. Hydrobiologia, 543:25-35, doi:10.1007/s10750-004-5713-2 [25, 39, 40, 116, 145, 147]

Bell, P. R. F., P. J. R. Uwins, I. Elmetri, J. A. Phillips, F. X. Fu, and A. J. E. Yago, 2005, Laboratory culture studies of Trichodesmium isolated from the Great Barrier Reef Lagoon, Australia. Hydrobiologia, 532:9-21 [24, 41]

Ben-Porath, J., E. J. Carpenter, and J. P. Zehr, 1993, Genotypic relationships in Trichodesmium (Cyanophyceae) based on nifH sequence comparisons. J. Phycol, 29:806810 [23]

Benincà, E., J. Huisman, R. Heerkloss, K. D. Jöhnk, P. Branco, E. H. Van Nes, M. Scheffer, and S. P. Ellner, 2008, Chaos in a long-term experiment with a plankton community. Nature, 451:822-826, doi:10.1038/nature06512 [28]

Benson, D. A., I. Karsch-Mizrachi, D. J. Lipman, J. Ostell, and D. L. Wheeler, 2005, Genbank. Nucleic Acids Res., 33:D34-D38, doi:10.1093/nar/gki063 [45, 74] 
Berman-Frank, I., K. D. Bidle, L. Haramaty, and P. G. Falkowski, 2004, The demise of the marine cyanobacterium, Trichodesmium spp., via an autocatalyzed cell death pathway. Limnol. Oceanogr., 49:997-1005 [90]

Berman-Frank, I., J. T. Cullen, Y. Shaked, R. M. Sherrell, and P. G. Falkowski, 2001, Iron availability, cellular iron quotas, and nitrogen fixation in Trichodesmium. Limnol. Oceanogr., 46:1249-1260 [121]

Berman-Frank, I., G. Rosenberg, O. Levitan, L. Haramaty, and X. Mari, 2007, Coupling between autocatalytic cell death and transparent exopolymeric particle production in the marine cyanobacterium Trichodesmium. Environ. Microbiol., 9:1415-1422, doi: 10.1111/j.1462-2920.2007.01257.x [90]

Bessetti, J., 2007, An introduction to PCR inhibitors [88]

Björkman, K. A., L. Thomson-Bulldis, and D. M. Karl, 2000, Phosphorus dynamics in the North Pacific subtropical gyre. Aquat. Microb. Ecol., 22:185-198 [137]

Breitbarth, E., M. M. Mills, G. Friedrichs, and J. LaRoche, 2004, The Bunsen gas solubility coefficient of ethylene as a function of temperature and salinity and its importance for nitrogen fixation assays. Limnol. Oceanogr.: Methods, 2:282-288 [126]

Breitbarth, E., A. Oschlies, and J. LaRoche, 2007, Physiological constraints on the global distribution of Trichodesmium-effect of temperature on diazotrophy. Biogeosciences, 4:53-61 [116, 147]

Campbell, L., E. J. Carpenter, J. P. Montoya, A. B. Kustka, and D. G. Capone, 2005, Picoplankton community structure within and outside a Trichodesmium bloom in the southwestern Pacific Ocean. Vie Milieu, 55:185-195 [122, 134, 135]

Capone, D. G., J. A. Burns, J. P. Montoya, A. Subramaniam, C. Mahaffey, T. Gunderson, A. F. Michaels, and E. J. Carpenter, 2005, Nitrogen fixation by Trichodesmium spp.: An important source of new nitrogen to the tropical and subtropical North Atlantic Ocean. Global Biogeochem. Cycles, 19:GB2024, doi:10.1029/2004GB002331 [21, $25,120,134,139]$

Capone, D. G. and J. P. Montoya, 2001, Nitrogen fixation and denitrification. In J. Paul (ed.), Methods in microbiology: marine microbiology, vol. 30, pp. 501-515, Academic Press, London, England [126]

Capone, D. G., J. Zehr, H. Paerl, B. Bergman, and E. J. Carpenter, 1997, Trichodesmium: A globally significant marine cyanobacterium. Science, 276:1221-1229 [20, 22, 31, 36, $72,90,120,144]$ 
Carpenter, E. J. and D. G. Capone, 1992, Nitrogen fixation in Trichodesmium blooms. In E. J. Carpenter, D. G. Capone, and J. G. Rueter (eds.), Marine pelagic cyanobacteria: Trichodesmium and other diazotrophs, pp. 211-217, Kluwer Academic Publishers, Dordrecht $[25,90]$

Carpenter, E. J., J. M. O’Neil, R. Dawson, D. G. Capone, P. J. A. Siddiqui, T. Roenneberg, and B. Bergman, 1993, The tropical diazotrophic phytoplankter Trichodesmium: biological characteristics of two common species. Mar. Ecol. Progr. Ser., 95:295-304 [31, 40, 115]

Carpenter, E. J. and C. C. Price, IV, 1977, Nitrogen fixation, distribution, and production of Oscillatoria (Trichodesmium) spp. in the western Sargasso and Caribbean Seas. Limnol. Oceanogr., 22:60-72 [25]

Carpenter, E. J., M. I. Scranton, P. C. Novelli, and A. Michaels, 1987, Validity of $\mathrm{N}_{2}$ fixation rate measurements in marine Oscillatoria (Trichodesmium). J. Plank. Res., 9:1047-1056 [90, 145]

Carpenter, E. J., A. Subramaniam, and D. G. Capone, 2004, Biomass and primary productivity of the cyanobacterium Trichodesmium spp. in the tropical N Atlantic Ocean. Deep Sea Res. I, 51:173-203 [21, 25, 72]

Chapman, J. S. and J. C. Meeks, 1987, Conditions for mutagenesis of the nitrogen-fixing cyanobacterium Anabaena variabilis. J. Gen. Microbiol., 113:111-118 [112]

Chappell, P. D., 2009, The relationship between iron and nitrogen fixation in Trichodesmium spp. Ph.D. thesis, Massachusetts Institute of Technology-Woods Hole Oceanographic Institution [93, 94, 95, 113, 114, 115, 146]

Church, M. J., B. D. Jenkins, D. M. Karl, and J. P. Zehr, 2005, Vertical distributions of nitrogen-fixing phylotypes at Stn ALOHA in the oligotrophic North Pacific Ocean. Aquat. Microb. Ecol., 38:3-14 [73, 91]

Cohen, Y. and M. Gurevitz, 2006, The cyanobacteria-ecology, physiology, and molecular genetics. In M. Dworkin, S. Falkow, E. Rosenberg, K. Schleifer, and E. Stackebrandt (eds.), The prokaryotes, vol. 4, p. 1074Đ1098, Springer, 3rd edn., doi:10.1007/0-38730744-3_39 [20, 87, 112]

Coles, V. J., R. R. Hood, M. Pascual, and D. G. Capone, 2004, Modeling the impact of Trichodesmium and nitrogen fixation in the Atlantic Ocean. J. Geophys. Res., 109:C06,007, doi:10.1029/2002JC001754 [22, 31, 120, 121]

Connell, J. H., 1961, The influence of interspecific competition and other factors on the distribution of the barnacle Chthamalus stellatus. Ecology, 42:710-723 [145] 
Cook, J., 1842, The Voyages of Captain James Cook, vol. 1. Bradbury and Evans, Printers, London [36]

Cotner, J. B., J. W. Ammerman, E. R. Peele, and E. Bentzen, 1997, Phosphorus-limited bacterioplankton growth in the Sargasso Sea. Aquat. Microb. Ecol., 13:141-149 [137]

Davis, C. S. and D. J. McGillicuddy, Jr., 2006, Transatlantic abundance of the $\mathrm{N}_{2}$-fixing colonial cyanobacterium Trichodesmium. Science, 312:1517-1520 [25, 30, 72, 91, 115]

Deutsch, C., N. Gruber, R. M. Key, and J. L. Sarmiento, 2001, Denitrification and $\mathrm{N}_{2}$ fixation in the Pacific Ocean. Global Biogeochem. Cycles, 15:483-506 [139]

Droop, M. R., 1974, The nutrient status of algal cells in continuous culture. J. Mar. Biol. Ass. U.K., 54:825-855 [27]

Drouet, F., 1968, Revision of the classification of the Oscillatoriaceae. Academy of Natural Sciences, Philadelphia, PA [38]

Dugdale, R. C. and J. J. Goering, 1967, Uptake of new and regenerated forms of nitrogen in primary productivity. Limnol. Oceanogr., 12:196-206 [120]

Dyhrman, S. T., P. D. Chappell, S. T. Haley, J. W. Moffett, E. D. Orchard, J. B. Waterbury, and E. A. Webb, 2006, Phosphonate utilization by the globally important marine diazotroph Trichodesmium. Nature, 439:68-71, doi:10.1038/nature04203 [22, 38, 113]

Dyhrman, S. T., E. A. Webb, D. A. Anderson, J. W. Moffett, and J. B. Waterbury, 2002, Cell-specific detection of phosphorus stress in Trichodesmium from the Western North Atlantic. Limnol. Oceanogr., 47:1832-1836 [122, 123, 125, 128, 138]

Eberl, R. and E. J. Carpenter, 2007, Association of the copepod Macrosetella gracilis with the cyanobacterium Trichodesmium spp. in the North Pacific Gyre. Mar. Ecol. Prog. Ser., 333:205-217 [145]

Ehrenberg, C. G., 1830, Neue beobachtungen über blauartige erscheinungen in Aegypten, Arabien und Sibirien, nebst einer übersicht und kritik der früher bekannten. Ann. Phys. Chem., 18:477-514 [36]

El-Shehawy, R., C. Lugomela, A. Ernst, and B. Bergman, 2003, Diurnal expression of het $R$ and diazocyte development in the Plamentous non-heterocystous cyanobacterium Trichodesmium erythraeum. Microbiol., 149:1139-1146 [32, 86, 145]

Eppley, R. W., 1972, Temperature and phytoplankton growth in the sea. Fish. B-NOAA, 70:1063-1085 [24]

Eppley, R. W. and B. J. Peterson, 1979, Particulate organic matter flux and planktonic new production in the deep ocean. Nature, 282:677-680 [21, 120] 
Follows, M. J., S. Dutkiewicz, S. Grant, and S. W. Chisholm, 2007, Emergent biogeography of microbial communities in a model ocean. Science, 315:1843-1846, doi: 10.1126/science.1138544 [30]

Foster, R. A., A. Paytan, and J. P. Zehr, 2009, Seasonality of $\mathrm{N}_{2}$ fixation and nifH gene diversity in the Gulf of Aqaba (Red Sea). Limnol. Oceanogr., 54:219-233 [94]

Foster, R. A., A. Subramaniam, C. Mahaffey, E. J. Carpenter, D. G. Capone, and J. P. Zehr, 2007, Influence of Amazon River plume on distributions of free-living and symbiotic cyanobacteria in the western tropical north Atlantic Ocean. Limnol. Oceanogr., 52:517-532 [73, 88, 91]

Foster, R. A. and J. P. Zehr, 2006, Characterization of diatom-cyanobactiera symbioses on the basis of nifH, hetR, and 16S rRNA sequences. Environ. Microbiol., 8:1913-1925, doi:10.1111/j.1462-2920.2006.01068.x. [74, 138]

Fung, I. Y., S. K. Meyn, I. Tegen, S. C. Doney, J. G. John, and J. K. B. Bishop, 2000, Iron supply and demand in the upper ocean. Global Biogeochem. Cycles, 14:281-295 [121, 140]

Gadagkar, S. R., M. S. Rosenberg, and S. Kumar, 2005, Inferring species phylogenies from multiple genes: concatenated sequence tree versus consensus gene tree. J. Exp. Zool. Part B, 304B:64-74 [45]

Geitler, L., 1932, Cyanophyceae. In L. Rabenhorst (ed.), Kryptogammenflora von Deutschland, Osterreich, under de Sweitz, vol. 14, pp. 673-1056, Akademische Verlagsgesellschaft, Leipzig [37, 38]

Glazer, A. N., 1987, Phycobilisomes: assembly and attachment. In P. Fay and C. Van Baalen (eds.), The Cyanobacteria, Elsevier, Amsterdam, the Netherlands [39]

Goebel, N. L., C. A. Edwards, M. J. Church, and J. P. Zehr, 2007, Modeled contributions of three types of diazotrophs to nitrogen fixation at Station ALOHA. ISME, 1:606-619 $[73,91,116]$

Golubic, S., 1977, Speciation in Trichodesmium: occupation of an oceanic pelagic niche. Schweiz. Z. Hydrol., 39:141-143 [38]

Gomont, M., 1892, Monographie des Oscillariées (Nostocacées homocystées), vol. ser. 7, vol. 15 of Annales des Sciences Naturelles, Botanique Series. Fortin, Masson, Paris [38]

González-Gil, S., B. A. Keafer, R. V. M. Jovine, A. Aguilera, S. Lu, and D. M. Anderson, 1998, Detection and quantification of alkaline phosphatase in single cells of phosphorus-starved marine phytoplankton. Mar. Ecol. Prog. Ser., 164:21-35 [123] 
Grover, J. P., 1990, Resource competition in a variable environment: phytoplankton growing according to Monod's model. Am. Nat., 136:771-789 [28]

Gruber, N. and J. Sarmiento, 1997, Global patterns of marine nitrogen fixation and denitrification. Global Biogeochem. Cycles, 11:235-266 [139]

Guiry, M. D. and G. M. Guiry, 2008, Algaebase. National University of Ireland, Galway, http://www.algaebase.org [37, 38]

Hall, B. G., 2004, Phylogenetic trees made easy: a how-to manual. Sinauer Associates, Inc., Sunderland, MA, 2nd edn. [70]

Hallegraeff, G. M., D. M. Anderson, and A. D. Cembella (eds.), 2003, Manual on harmful marine microalgae. UNESCO, Paris [38]

Herdman, M., M. Janvier, R. Rippka, and R. Y. Stanier, 1979, Genome size of cyanobacteria. J. Gen. Microbiol., 111:73-85 [87, 112]

Hewson, I., S. R. Govil, D. G. Capone, and E. J. Capone, 2004, Evidence of Trichodesmium viral lysis and potential significance for biogeochemical cycling in the oligotrophic ocean. Aquat. Microb. Ecol., 36:1-8 [90, 116]

Hewson, I., R. S. Poretsky, S. T. Dyhrman, B. Zielinski, A. E. White, H. J. Tripp, J. P. Montoya, and J. P. Zehr, 2009, Microbial community gene expression within colonies of the diazotroph, Trichodesmium, from the Southwest Pacific Ocean. ISME, doi: 10.1038/ismej.2009.75 [36]

Hoffmann, L., 1988, Criteria for the classification of blue-green algae (cyanobacteria) at the genus and at the species level. Arch. Hydrobiol. Suppl., 80:131-139 [36]

Holl, C. M. and J. P. Montoya, 2005, Interactions between nitrate uptake and nitrogen fixation in continuous cultures of the marine diazotroph Trichodesmium (Cyanobacteria). J. Phycol., 41:1178-1183 [116]

Hood, R. R., A. M. Michaels, and D. G. Capone, 2000, Answers sought to the enigma of marine nitrogen fixation. EOS Trans. Am. Geophys. Union, 81:138-139 [120]

Huisman, J., A. M. Johansson, E. O. Folmer, and F. J. Weissing, 2001, Towards a solution of the plankton paradox: the importance of physiology and life history. Ecol. Lett., 4:408-411 [29]

Huisman, J. and F. J. Weissing, 1999, Biodiversity of plankton by species oscillations and chaos. Nature, 402:407-410 [28] 
Hutchins, D. A., F. X. Fu, Y. Zhang, M. E. Warner, Y. Feng, K. Portune, P. W. Bernhardt, and M. R. Mulholland, 2007, $\mathrm{CO}_{2}$ control of Trichodesmium $\mathrm{N}_{2}$ fixation, photosynthesis, growth rates, and elemental ratios: Implications for past, present, and future ocean biogeochemistry. Limnol. Oceanogr., 52:1293-1304 [116, 147]

Hutchinson, G. E., 1957, Concluding remarks. Cold Spring Harb. Sym., 22:415-427 [29]

—, 1961 , The paradox of the plankton. Am. Nat., 54:137-145 [19, 26]

Hynes, A. M., P. D. Chappell, S. T. Dyhrman, S. C. Doney, and E. A. Webb, 2009, Crossbasin comparison of phosphorus stress and nitrogen fixation in Trichodesmium. Limnol. Oceanogr., 54:1438-1448 [30, 37, 93, 95, 144]

Janson, S., B. Bergman, E. J. Carpenter, S. J. Giovannoni, and K. Vergin, 1999, Genetic analysis of natural populations of the marine diazotrophic cyanobacterium Trichodesmium. FEMS Microbiol. Ecol., 30:57-65 [45, 46, 47, 67, 86, 107, 108, 109, 114, $115,144,147]$

Janson, S., P. J. A. Siddiqui, A. E. Walsby, K. M. Romans, E. J. Carpenter, and B. Bergman, 1995, Cytomorphological characterization of the planktonic diazotrophic cyanobacteria Trichodesmium spp. from the Indian Ocean and Caribbean and Sargasso Seas. J. Phycol., 31:463-477 [23, 24, 36, 37, 39, 66]

Johnson, Z. I., E. R. Zinser, A. Coe, N. P. McNulty, E. Malcolm, S. Woodward, and S. W. Chisholm, 2006, Niche partitioning among Prochlorococcus ecotypes along oceanscale environmental gradients. Science, 311:1737-1740, doi:10.1126/science.1118052 $[30,73]$

Karl, D., R. Letelier, L. Tupas, J. Dore, J. Christian, and D. Hebel, 1997, The role of nitrogen fixation in biogeochemical cycling in the subtropical North Pacific Ocean. $\mathrm{Na}$ ture, 388:533-538 [21, 121, 134, 139]

Karl, D., A. Michaels, B. Bergman, D. Capone, E. Carpenter, R. Letelier, F. Lipshultz, H. Paerl, D. Sigman, and L. Stal, 2002, Dinitrogen fixation in the world's oceans. Biogeochem., 57-58:47-98 [136]

Karl, D. M., 2002, Nutrient dynamics in the deep blue sea. Trends Microbiol., 10:410-418 $[21,22,24,121,140]$

Karl, D. M., R. M. Letelier, D. V. Hebel, D. F. Bird, and C. D. Winn, 1992, Trichodesmium blooms and new nitrogen in the North Pacific gyre. In E. J. Carpenter, D. G. Capone, and J. G. Rueter (eds.), Marine pelagic Cyanobacteria: Trichodesmium and other diazotrophs, pp. 219-237, Kluwer Academic Publishers, Norwell, MA [116]

Kot, M., 2001, Elements of Mathematical Ecology. Cambridge University Press, Cambridge, UK [26] 
Kovesi, P., 1999, Phase preserving denoising of images. The Australian Pattern Recognition Society Conference: DICTA'99 [76]

Krauk, J. M., T. A. Villareal, J. A. Sohm, J. P. Montoya, and D. G. Capone, 2006, Plasticity of N:P ratios in laboratory and field populations of Trichodesmium spp. Aquat. Microb. Ecol., 42:243-253 [134, 137]

Kustka, A. B., S. A. Sañudo-Wilhelmy, E. J. Carpenter, D. Capone, J. Burns, and W. G. Sunda, 2003, Iron requirements for dinitrogen- and ammonium-supported growth in cultures of Trichodesmium (IMS101): Comparison with nitrogen fixation rates and iron:carbon ratios of field populations. Limnol. Oceanogr., 48:1869-1884 [22, 137]

Kützing, F. T., 1845, Phycologia germanica, d. i. Deutschlands Algen in bündigen Beschreibungen. Nebst einer Anleitung zum Untersuchen und Bestimmen dieser Gewächse für Anfänger. Nordhausen, W. Köhne [38]

LaRoche, J. and E. Breitbarth, 2005, Importance of the diazotrophs as a source of new nitrogen in the ocean. J. Sea Res., 53:67-91, doi:10.1016/j.seares.2004.05.005 [90, 112]

Letelier, R. M. and D. M. Karl, 1996, Role of Trichodesmium spp. in the productivity of the subtropical North Pacific Ocean. Mar. Ecol. Prog. Ser., 133:263-273 [25, 72, 90]

Levins, R., 1979, Coexistence in a variable environment. Am. Nat., 114:765-783 [26]

Li, M. G., B. Z. Bian, R. A. Lewin, L. C. L, and Z. N. Pan, 1984, Laboratory culture of a new species of planktonic blue-green alga, Trichodesmium maccii, from the Qingdao Coast, China. J. Shandong Coll. Oceanol., 14:93-101 [38]

Lugomela, C., T. J. Lyimo, I. Bryceson, A. K. Semesi, and B. Bergman, 2002, Trichodesmium in coastal waters of Tanzania: diversity, seasonality, nitrogen and carbon fixation. Hydrobiologia, 477:1-13 [25]

Lundgren, P., S. Janson, S. Jonasson, A. Singer, and B. Bergman, 2005, Unveiling of novel radiations within Trichodesmium cluster by hetR gene sequence analysis. Appl. Environ. Microbiol., 71:190-196, doi:10.1128/AEM.71.1.190-196.2005 [23, 24, 32, $37,38,39,41,43,44,45,47,67,86,107,108,114,144]$

Lundgren, P., E. Söderbäck, A. Singer, E. J. Carpenter, and B. Bergman, 2001, Katagnymene: characterization of a novel marine diazotroph. J. Phycol., 37:1052-1062 [37, $67,86,145]$

Martiny, A. C., S. Kathuria, and P. M. Berube, 2009, Widespread metabolic potential for nitrite and nitrate assimilation among Prochlorococcus ecotypes. PNAS, 106:10,78710,792, doi:10.1073/pnas.0902532106 [30] 
Mather, R. L., S. E. Reynolds, G. A. Wolff, R. G. Williams, S. Torres-Valdes, E. M. S. Woodward, A. Landolfi, X. Pan, R. Sanders, and E. P. Achterberg, 2008, Phosphorus cycling in the North and South Atlantic Ocean subtropical gyre. Nature Geosci., 1:439$443[113,122,136,140]$

McCarthy, J. J. and E. J. Carpenter, 1979, Oscillatoria (Trichodesmium) thiebautii (cyanophyta) in the central north Atlantic Ocean. J. Phycol., 15:75-82 [40]

Mes, T. H. M. and L. J. Stal, 2005, Variable selection pressures across lineages in Trichodesmium and related cyanobacteria based on the heterocyst differentiation protein gene hetR. Gene, 346:163-171 [145]

Mills, M., C. Ridame, M. Davey, J. L. Roche, and R. J. Geider, 2004, Iron and phosphorus co-limit nitrogen fixation in the eastern tropical North Atlantic. Nature, 429:292-294 [121]

Moore, J. K. and O. Braucher, 2008, Sedimentary and mineral dust sources of dissolved iron to the world ocean. Biogeosci., 5:631-656 [113, 122, 140]

Moore, J. K. and S. C. Doney, 2007, Iron availability limits the ocean nitrogen inventory stabilizing feedbacks between marine denitrification and nitrogen fixation. Global Biogeochem. Cycles, 21:GB2001, doi:10.1029/2006GB002762 [22, 121, 139]

Moore, J. K., S. C. Doney, D. M. Glover, and I. Y. Fung, 2002, Iron cycling and nutrientlimitation patterns in surface waters of the World Ocean. Deep Sea Res II, 49:463-507 [22]

Moore, J. K., S. C. Doney, and K. Lindsay, 2004, Upper ocean ecosystem dynamics and iron cycling in a global three-dimensional model. Global Biogeochem. Cycles, 18:GB4028, doi:10.1029/2004GB002220 [31, 90, 116, 120, 121, 139, 140]

Moutin, T., N. Van Den Broeck, B. Beker, C. Dupouy, P. Rimmelin, and A. L. Bouteiller, 2005, Phosphate availability controls Trichodesmium spp. biomass in the SW Pacific Ocean. Mar. Ecol. Prog. Ser., 297:15-21 [136]

Mulholland, M. R., S. Floge, E. J. Carpenter, and D. G. Capone, 2002, Phosphorus dynamics in cultures and natural populations of Trichodesmium spp. Mar. Ecol. Prog. Ser., 239:45-55 [116, 134, 139]

Neveux, J. and M. M. B. Tenório, 2008, Response to "Another look at green Trichodesmium colonies". Limnol. Oceanogr., 53:2052-2055 [69]

Neveux, J., M. M. B. Tenório, C. Dupouy, and T. A. Villareal, 2006, Spectra diversity of phycoerythrins and diazotroph abundance in tropical waters. Limnol. Oceanogr., 51:1689-1698 [25, 40, 68, 69] 
Nübel, U., F. Garcia-Pichel, and G. Muyzer, 1997, PCR primers to amplify 16S rRNA genes from cyanobacteria. Appl. Environ. Microbiol., 63:3327-3332 [43, 44]

O’Neil, J. M., 1998, The colonial cyanobacterium Trichodesmium as a physical and nutritional substrate for the harpacticoid copepod it Macrosetella gracilis. J. Plankton Res., 20:43-59 [23, 25, 145]

O’Neil, J. M. and M. R. Roman, 1994, Ingestion of the cyanobacterium Trichodesmium spp. by pelagic harpacticoid copepods Macrosetella, Miracia and Oculosetella. Hydrobiologia, 292/293:235-240 [23, 30, 36, 116, 145]

Orcutt, K. M. and K. Gundersen, 2003, Seasonal and spatial distribution of Trichodesmium spp. at the Bermuda Atlantic Time-series Study (BATS) site. Poster at: Open Science Conference, U. S. Joint Global Ocean Flux Study [24, 30]

Orcutt, K. M., K. Gundersen, and U. Rasmussen, 2008, Another look at green Trichodesmium colonies. Limnol. Oceanogr., 53:2049-2051 [69]

Orcutt, K. M., U. Rasmussen, E. A. Webb, J. B. Waterbury, K. Gundersen, and B. Bergman, 2002, Characterization of Trichodesmium spp. by genetic techniques. Appl. Environ. Microbiol., 68:2236-2245 [23, 24, 37, 41, 43, 44, 45, 67]

Post, A. F., 2005, Nutrient limitation of marine cyanobacteria: Molecular ecology of nitrogen limitation in an oligotrophic sea. In J. Huisman, H. C. P. Matthijs, and P. M. Visser (eds.), Harmful Cyanobacteria, vol. 3 of Aquatic Ecology Series, pp. 87-107, Springer, Dordrecht, The Netherlands [22, 23]

Post, A. F., Z. Dedej, R. Gottlieb, H. Li, D. N. Thomas, M. El-Absawi, A. El-Naggar, M. El-Gharabawi, and U. Sommer, 2002, Spatial and temporal distribution of Trichodesmium spp. in the stratified Gulf of Aqaba, Red Sea. Mar. Ecol. Prog. Ser., 239:241-250 [25, 30, 72, 90, 115]

Prufert-Bebout, L., H. W. Paerl, and C. Lassen, 1993, Growth, nitrogen fixation, and spectral attenuation in cultivated Trichodesmium species. Appl. Env. Micribiol., 59:1367$1375[41,67]$

Rabouille, S., M. Staal, L. J. Stal, and K. Soetaert, 2006, Modeling the dynamic regulation of nitrogen fixation in the cyanobacterium Trichodesmium sp. Appl. Environ. Microbiol., 72:3217-3227, doi:10.1128/AEM.72.5.3217-3227.2006 [116]

Radström, P., C. Löfström, M. Lövenklev, R. Knutsson, and P. Wolffs, 2008, Stragegies for overcoming PCR inhibition. Cold Spring Harb. Protoc. [88]

Revilla, T. and F. J. Weissing, 2008, Nonequilibrium coexistence in a competition model with nutrient storage. Ecology, 89:865-877 [28] 
Rippka, R., J. Deruelles, J. B. Waterbury, M. Herdman, and R. Y. Stanier, 1979, Generic assignments, strain histories, and properties of pure cultures of cyanobacteria. J. Gen. Microbiol., 111:1-61 [36, 37, 138]

Rippka, R., J. Waterbury, and G. Cohen-Bazire, 1974, A cyanobacterium which lacks thylakoids. Arch. Microbiol., 100:419-436 [39, 42]

Rocap, G., D. L. Distel, J. B. Waterbury, and S. W. Chisholm, 2002, Resolution of Prochlorococcus and Synechococcus ecotypes by using 16S-23S ribosomal DNA internal transcribed spacer sequences. Appl. Environ. Microbiol., 68:1180-1191 [43, 44]

Rocap, G., F. W. Larimer, J. Lamerdin, S. Malfatti, P. Chain, N. A. Ahlgren, A. Arellano, M. Coleman, L. Hauser, W. R. Hess, Z. I. Johnson, M. Land, D. Lindell, A. F. Post, W. Regala, M. Shah, S. L. Shaw, C. Steglich, M. B. Sullivan, C. S. Ting, A. Tolonen, E. A. Webb, E. R. Zinser, and S. W. Chisholm, 2003, Genome divergence in two Prochlorococcus ecotypes reflects oceanic niche differentiation. Nature, 424:10421046 [30]

Sakr, S., M. Thyssen, M. Denis, and C.-C. Zhang, 2006, Relationship among several key cell cycle events in the developmental cyanobacterium Anabaena sp. strain PCC 7120. J. Bacteriol., 188:5958-5965, doi:10.1128/JB.00524-06 [112]

Sandh, G., R. El-Shehawy, B. Díez, and B. Bergman, 2009, Temporal separation of cell division and diazotrophy in the marine diazotrophic cyanobacterium Trichodesmium erythraeum IMS101. FEMS Microbiol. Lett., 295:281-288 [87]

Sañudo-Wilhelmy, S. A., A. B. Kustka, C. J. Gobler, D. A. Hutchins, M. Yang, K. Lwiza, J. Burns, D. G. Capone, J. A. Raven, and E. J. Carpenter, 2001, Phosphorus limitation of nitrogen fixation by Trichodesmium in the central Atlantic Ocean. Nature, 411:6669 [121, 139]

Schopf, J. W., 2000, The fossil record: tracing the roots of the cyanobacterial lineage. In B. A. Whitton and M. Potts (eds.), The ecology of cyanobacteria, pp. 13-35, Kluwer Academic Publishers, Dordrecht, The Netherlands [20]

Schütt, F., 1893, Der Pflanzenleben der Hochsee. Lipsius and Tischer, Kiel, Leipzig [38]

Sheridan, C. C., D. K. Steinberg, and G. W. Kling, 2002, The microbial and metazoan community associated with colonies of Trichodesmium spp.: a quantitative survey. $J$. Plankton Res., 24:913-922 [22, 36, 138]

Shigesada, N. and K. Kawasaki, 1997, Biological Invasions: Theory and Practice. Oxford University Press, Oxford [27]

Short, S. M., B. D. Jenkins, and J. P. Zehr, 2004, Spatial and temporal distribution of two diazotrophic bacteria in the Chesapeake Bay. Appl. Environ. Microbiol., 70:2186-2192 [94] 
Siddiqui, P. J. A., B. Bergman, and E. J. Carpenter, 1992, Filamentous cyanobacterial associates of the marine planktonic cyanobacterium Trichodesmium. Phycologia, 31:326337 [138]

Siegel, D. A., 1998, Resource competition in a discrete environment: why are plankton distributions paradoxical? Limnol. Oceanogr., 43:1133-1146 [29]

Silva, P. C., P. W. Basson, and R. L. Moe, 1996, Catalogue of the benthic marine algae of the Indian Ocean, vol. 79 of University of California Publications in Botany. University of California Press [38]

Sohm, J. A. and D. G. Capone, 2006, Phosphorus dynamics of the tropical and subtropical north Atlantic: Trichodesmium spp. versus bulk plankton. Mar. Ecol. Prog. Ser., 317:21-28 [22, 113, 122, 134, 139]

Sohm, J. A., C. Mahaffey, and D. G. Capone, 2008, Assessment of relative phosphorus limitation of Trichodesmium spp. in the North Pacific, North Atlantic, and the north coast of Australia. Limnol. Oceanogr., 53:2495-2502 [134, 136, 137, 139]

Stihl, A., U. Sommer, and A. F. Post, 2001, Alkaline phosphatase activities among populations of the colony-forming diazotrophic cyanobacterium Trichodesmium spp. (cyanobacteria) in the Red Sea. J. Phycol., 37:310-317 [30, 33, 122]

Stomp, M., J. Huisman, F. de Jongh, A. J. Veraart, D. Gerla, M. Rijkeboer, B. W. Ibelings, U. I. A. Wollenzien, and L. J. Stal, 2004, Adaptive divergence in pigment composition promotes phytoplankton biodiversity. Nature, 432:104-107 [29]

Subramaniam, A., E. J. Carpenter, D. Karentz, and P. G. Falkowski, 1999, Bio-optical properties of the marine diazotrophic cyanobacteria Trichodesmium spp. I. Absorption and photosynthetic action spectra. Limnol. Oceanogr. 44:608-617 [39, 68, 69, 145]

Sweeney, E. N., J. McGillicuddy, D. J., and K. O. Buesseler, 2003, Biogeochemical impacts due to mesoscale eddy activity in the Sargasso Sea as measured at the Bermuda Atlantic Time-series Study (BATS). Deep Sea Res. II, 50:3017-3039 [25]

Templeton, K. E. and E. C. J. Claas, 2003, Comparison of four real-time PCR detection systems: Bio-Rad I-Cycler, ABI 7700, Roche LightCycler, and the Cepheid Smartcycler. In C. W. Dieffenbach and G. S. Dveksler (eds.), PCR Primer: a Laboratory Manual, pp. 187-195, Cold Spring Harbor Laboratory Press, Cold Spring Harbor, NY, 2nd edn. [73]

Tillett, D. and B. A. Neilan, 2000, Xanthogenate nucleic acid isolation from cultured and environmental cyanobactera. J. Phycol., 36:251-258 [78, 87, 88]

Tilman, D., 1977, Resource competition between plankton algae: an experimental and theoretical approach. Ecology, 58:338-348 [26, 27, 28, 113] 
Tilman, D., M. Mattson, and S. Langer, 1981, Competition and nutrient kinetics along a temperature gradient: an experimental test of a mechanistic approach to niche theory. Limnol. Oceanogr., 26:1010-1033 [26, 27, 29]

Titman, D., 1976, Ecological competition between algae: experimental confirmation of resource-based competition theory. Science, 192:463-465 [22, 26]

Toledo, G., B. Palenik, and B. Brahamsha, 1999, Swimming marine Synechococcus strains with widely different photosynthetic pigment ratios form a monophyletic group. Appl. Environ. Microbiol., 65:5247-5251 [67]

Tuit, C., J. Waterbury, and G. Ravizza, 2004, Diel variation of molybdenum and iron in marine diazotrophic cyanobacteria. Limnol. Oceanogr., 49:978-990 [116]

Tyrrell, T., 1999, The relative influences of nitrogen and phosphorus on oceanic primary production. Nature, 400:525-531 [140]

Tyrrell, T., E. Marañón, A. J. Poulton, A. R. Bowie, D. S. Harbour, and E. M. S. Woodward, 2003, Large-scale latitudinal distribution of Trichodesmium spp. in the Atlantic Ocean. J. Plankton Res., 25:405-416 [24, 72, 90]

Van Den Broeck, N., T. Moutin, M. Rodier, and A. L. Bouteiller, 2004, Season variations of phosphate availability in the SW Pacific Ocean near New Caledonia. Mar. Ecol. Prog. Ser., 268:1-12 [122, 137]

Walsby, A. E., 1992, The gas vesicles and buoyancy of Trichodesmium. In E. J. Carpenter, D. G. Capone, and J. G. Rueter (eds.), Marine pelagic cyanobacteria: Trichodesmium and other diazotrophs, pp. 141-161, Kluwer Academic Publishers, Dordrecht [146]

Waterbury, J. B., 2006, The cyanobacteria-isolation, purification and identification. In M. Dworkin, S. Falkow, E. Rosenberg, K. Schleifer, and E. Stackebrandt (eds.), The prokaryotes, vol. 4, pp. 1053-1073, Springer, 3rd edn., doi:10.1007/0-387-30744-3_38 $[20,23,138]$

Waterbury, J. B., S. W. Watson, F. W. Valois, and D. G. Franks, 1986, Biological and ecological characterization of the marine unicellular cyanobacterium Synechococcus. In Photosynthetic Picoplankton, vol. 214 of Can. Bull. Fish. Aquatic Sci., pp. 71-120, Department of Fisheries and Oceans, Ottawa, Canada [31]

Webb, E. A., R. W. Jakuba, J. W. Moffett, and S. T. Dyhrman., 2007, Molecular assessment of phosphorus and iron physiology in Trichodesmium populations from the western Central and western South Atlantic. Limnol. Oceanogr., 52:2221-2232 [30, 123, $136,138,139,140]$ 
Webb, E. A., J. W. Moffett, and J. B. Waterbury, 2001, Iron stress in open-ocean cyanobacteria (Synechococcus, Trichodesmium, and Crocosphaera spp.): Identfication of the IdiA protein. Appl. Environ. Microbiol., 67:5444-5452, doi: 10.1128/AEM.67.12.5444-5452.2001 [42]

White, A. E., Y. H. Spitz, D. M. Karl, and R. M. Letelier, 2006a, Flexible elemental stoichiometry in Trichodesmium spp. and its ecological implications. Limnol. Oceanogr., 51:1777-1790 [22, 134, 137]

White, A. E., Y. H. Spitz, and R. M. Letelier, 2006b, Modeling carbohydrate ballasting by Trichodesmium spp. Mar. Ecol. Prog. Ser., 323:35-45 [22, 116]

Whitton, B. A. and M. Potts, 1982, Marine littoral. In N. G. Carr and B. A. Whitton (eds.), The Biology of Cyanobacteria, vol. 19, pp. 515-542, University of California Press [138]

Wille, N., 1904, Die schizophyceen der plankton-expedition. In V. Hensen (ed.), Ergebnisse der Plankton-Expedition der Humbolt-Stiftung, vol. 4, Lipsius and Tischer, Kiel [38]

Wu, J., W. Sunda, E. A. Boyle, and D. M. Karl, 2000, Phosphate depletion in the western North Atlantic Ocean. Science, 289:759-762 [90, 113, 121, 122, 134, 137, 139]

Zinser, E. R., A. Coe, Z. I. Johnson, A. C. Martiny, N. J. Fuller, D. J. Scanlan, and S. W. Chisholm, 2006, Prochlorococcus ecotype abundances in the North Atlantic Ocean as revealed by an improved quantitative PCR method. Appl. Eviron. Microbiol., 72:723732, doi:10.1128/AEM.72.1.723-732.2006 [30, 73, 75, 77, 81] 